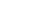

National Conference on

\title{
RECENT ADVANCES IN COMMUNICATION ENGINEERING AND INFORMATION TECHNOLOGY
}

$6^{\text {th }} \& 7^{\text {th }}$ December, 2019

Conference Chair :

Dr.Vinay kumar Mittal,

Dr.V.S.V.Prabhakar

Conference Co-Chair :

Dr. I. Govardhani 


\section{KL Deemed to be University}

\section{National Conference on Recent Advances in Communication Engineering and Information Technology}

\section{Proceedings of NCRACEIT-2019}

$$
6^{\text {th }} \& 7^{\text {th }} \text { December } 2019
$$

Organized by

Communication Systems Research Group

Department of Electronics and Communication Engineering (DST FIST

Sponsored), Koneru Lakshmaiah Education Foundation, AP, India

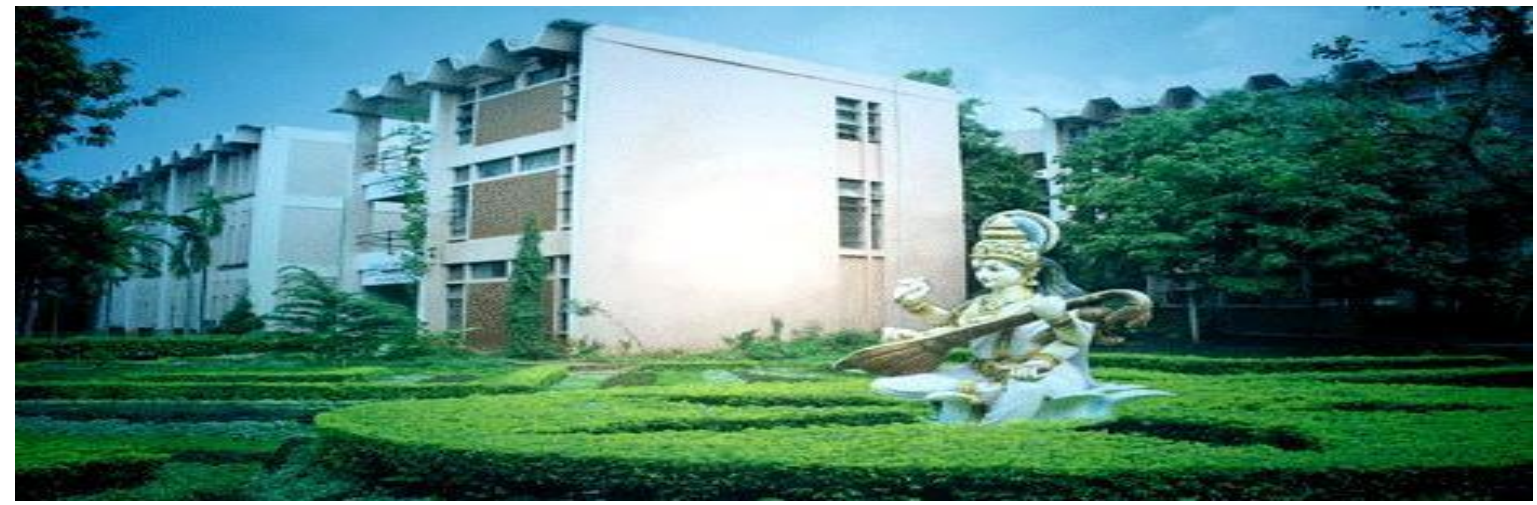

ISBN: ****** 


\section{National Conference on Recent Advances in Communication Engineering and Information Technology}

$6^{\text {th }} \& 7^{\text {th }}$ December 2019

CHAIRMAN

Dr. M SUMAN

\section{CO-CHAIRMAN}

Dr. M Venkata Narayana, Dy. Head, ECE

Dr. M Siva Ganga Prasad, Dy. Head, ECE

Dr. Lakshman Pappula, Dy. Head, ECE

\section{CONVENER}

Dr. Habibulla Khan, Professor, Dean (SA)

\section{CO ORDINATORS}

Dr. D Venkata Ratnam, Professor ECE, K L (Deemed to be University)

Dr., B. T. P. Madhav, Professor ECE, K L (Deemed to be University) Mr. K T P S Kumar, Asst. Professor ECE, K L (Deemed to be University) Mr. K V K V L Pavan Kumar, Asst. Professor ECE, K L (Deemed to be University)

Organised by:

Communication Systems Research Group

Department of ECE 


\section{Department of Electronics and Communication Engineering (DST-FIST Sponsored)}

\section{Department Profile}

K L Deemed to be University's Electronics and Communication Engineering Department was established in the year 1980, which is one of the Sphere Heads with a Faculty Strength of 170 plus and over 2000 Under Graduate, 200 Post Graduate students and $180 \mathrm{Ph} . \mathrm{D}$ scholars. It is a well-established and well equipped department with novel teaching methodologies such as Lab Taken to Class (LTC), Project Based Labs (PBL) so on and so forth. The Department has various state-of-art laboratories including a Computer centres, Antenna test facility etc., which are well equipped to the highest level of sophistication and cater to the needs of UG, PG and Ph.D students. Department's academic calendar is highlighted by regular seminars, group discussions, student paper contests, Industry alliance and Student Personality Development Programs for all round development of students in National \& International events. The Department has organized four International conferences under the aegis of RSPS - 2008 and RSPS - 2010. As the largest academic department of University, Department of ECE and its Faculty are involved in an exceptional range of Educational, Intellectual and Research activities. It has grown over the years in many directions and currently recognized as a "Premier Teaching and Research Department" of the KL University and also got recognition by DST under FIST program in the year 2012.

Our first mission is to educate Electronics and Communication engineers for Indian and Global industries. Our philosophy is not only to educate engineers, but also to prepare them for future leadership roles in industry- indeed, many of our alumni are now Directors of Industry, leading their organizations as Chief Executive Officers and Managing Directors. We provide a broad-based engineering curriculum, with opportunities for specialization and self-directed learning and development. We invest much effort to enhance our student's learning experience through the application of ECE technology.

Our second mission is to strive for research leadership in selected strategic research areas that are relevant to the National strategies for Science and Technology. Our department encourages multidisciplinary and collaborative approach to research to solve major research challenges of Societal and Global significance. Faculty members engage in projects with investigators from other schools within the campus, and other National and International Institutions and Agencies. Our third mission is to focus on commercial applications for the research we carry out-through Patents, Licenses or spin off companies.

The Department of ECE offers various specialization courses such as Communication Systems, Control Systems, Embedded Systems, Networking and Communication, Robotics and Mechatronics, Signal Processing and VLSI for both UG, PG and Doctor of Philosophy (Ph.D) degrees. 


\title{
Organizing Committee
}

\author{
Chief Patron \\ Sri Koneru Satyanarayana, President, KLEF
}

Patrons

Sri K. Lakshman Havish, Vice President, KLEF

Sri K. Raja Harin, Vice President, KLEF

Dr. S.S. Mantha, Chancellor, K L (Deemed to be University)

Dr. L. S. S. Redddy, Vice Chancellor, K L (Deemed to be University)

Dr. A Rama Kumar, Pro-Vice Chancellor, K L (Deemed to be University)

Dr. R.R.L. Kantam, Registrar, K L (Deemed to be University)

Conference Chair

Dr. Vinay Kumar Mittal, Professor ECE, K L (Deemed to be University)

Dr. V. S. V. Prabhakar, Professor ECE, K L (Deemed to be University)

\section{Conference Co-Chair}

Dr. I Govardhini, Professor ECE, K L (Deemed to be University)

Conference \& Technical Advisory Committee

Dr. Vijay Janyani, Professor, MNIT, Jaipur, India

Dr. Ganapati panda, Professor, IIT, Bhubaneswar, India

Dr. C. S. Sastry, Professor, IIT Hyderabad

Dr. K. N. Bhat, Scientist-H, IISC, Bangalore

Dr. Bidyadhar Subudhi, Professor, NIT, Rourkela

Dr. K. Lal Kishore, Former Vice-chancellor, JNTU-A

Dr. B. Rajesh Kumar Kaushik, Assoc. Professor, IIT, Roorkee

Dr. K. Sarat Kumar, Professor, ECE, K L (Deemed to be University)

K. Ch. Sri Kavya, Professor ECE, K L (Deemed to be University)

Dr. K. Kumar Naik, Professor, ECE, K L (Deemed to be University)

Dr. P. V. V. Kishore, Professor, ECE, K L (Deemed to be University)

Dr. G. V. Subbarao, Professor, ECE, K L (Deemed to be University)

Dr. P Parthasaradhi, Professor, ECE, K L (Deemed to be University)

Dr. K. Srinivasa Rao, Professor, ECE, K L (Deemed to be University)

Dr. S. Koteswara Rao, Professor, ECE, K L (Deemed to be University)

Dr. K S Ramesh, Professor, ECE, K L (Deemed to be University)

Dr. K Hari Kishore, Professor, ECE, K L (Deemed to be University)

Dr. V Rajesh, Professor, ECE, K L (Deemed to be University)

Dr. M Venu Gopala Rao, Professor, ECE, K L (Deemed to be University)

Dr. M. Z. Rehman, Professor, ECE, K L (Deemed to be University)

Dr. P Satya Narayana, Professor, ECE, K L (Deemed to be University)

Dr. Madhukar Deshmukh, Professor, ECE, K L (Deemed to be University)

Dr. ASCS Ssastry, Professor, ECE, K L (Deemed to be University) 


\section{INDEX}

1 Design of Elliptical Planar Monopole Antenna for UWB Applications

A Multilayer Antenna with Stacked Structure for ISM Applications

3 Detailed Analysis on the Atmospheric Effects on FSO Links at 405nm

4 Design of Linear Rectangular Microstrip Antenna for Ka Band Applications

5 A Novel Method to Detect OSA Using Deep Convolution Neural Network

6 Analytics Models for Diabetes Prediction Using a Machine Learning

7 Application of Advanced Statistical Signal Processing Algorithms for Prognostic Studies on Earthquakes Using Ultra Low Frequency Geomagnetic Signals

8 Study of Physical Phenomenon of Seismo-Ionospheric Perturbations Using GPSTEC - A New Perspective

9 Development of Blink Restoration Model for Facial Paralysis Detection

10 Comparative Analysis of Watermarking Methods on CFRP Sample Thermal Images

11 Genomic Analysis Using Higher Order Adaptive Exon Predictors

12 Efficient Cardiac Signal Enhancement Techniques Based on Variable Step Size and Data Normalized Hybrid Signed Adaptive Algorithms

13 Classification of Dress Codes Using CNN

14 Random Number Generator Using LABVIEW

algorithms

13
Using Modified NLMS Algorithm

16 An Efficient Optimal Threshold-Based Segmentation and Classification Model for Multi-Level Spinal Cord Injury Detection

17 An Early 3d Human Action Recognition (3Dhar) System by Training Sub Actions of an Action Using Deep Networks

18 A Quad Joint Relational Feature for 3D Skeletal Action Recognition with Circular CNNS

19 A Real Time Prototype Model for the Security Features in the ATM

Networks and its Application Specific Challenges

23 Model Assessment of Ionospheric Variability over Cyprus

24 Ionospheric Total Electron Content Variation over Saudi Arabian Region During 6-10 September 2017 Solar Flares and Associated Geomagnetic Storms

25 Analysis of Non-Stationary Signals by Stock well Transform

26 Time Frequency Analysis of Non-Stationary Signals by Differential Frequency Window S-Transform

27 Analysis of an Energy-Balanced Underwater Acoustic Sensor Networks 
30 BER Analysis of Communication System in the Scattering Scenario at

Mm-Wave Frequency Range

31 Synthesis of Unequally Spaced Linear Antenna Array Using Modified Cat

Swarm Optimization

32 Null Synthesis of Linear Antenna Array Using Gaussian Mutated Cat

Swarm Optimization

33 Fuzzy Utilization in Speech Recognition and its Different Application

Design of Magnitude Comparator Using Adiabatic Logic in ECRL

Design of XOR And XNOR Gates Based on Full Adder Circuits

37 Evaluation of Adverse Effects of Fog on FSO Links

38 Understanding the Effects of Various Modulation Techniques on FSO 38

Links

39 Reduction of Peak to Average Power Ratio in OFDM System by Using

Selected Mapping Technique

40 Stochastic-Geometry Based Characterization of Aggregate Interference in TVWS Cognitive Radio Networks

41 Extreme Eigenvalue Based Detection Under Impact of Noise and Interference Uncertainty

42 Machine Learning Based Subsurface Analysis Chirp-Z Transform Based Defect Detection

Gradient Pyramid Based Enhanced Defect Detection for Quadratic

Frequency Modulated Thermal Wave Imaging

Principle Component Analysis for Non-Stationary Thermal Wave Imaging

Impact of Light Soaking on Absorber and Buffer Layer in Thin Film Solar Cells

47 Impact of Selenium Composition Variation in CZTS Solar Cells

50 A Matlab Approach for Design Of Virtual Footwear

51 Design of Mems Based Capacitive Pressure Sensorto Monitor The 51

52 Impact of Ground Etching, Substrate And Inset Feed Height On Circularly Shaped Patch Antenna

53 Chexnet Reimplementation for Pneumonia Recognition Utilizing Pytorch

55 Home-Appliances Monitoring Based Wireless Gateway Network Using Packet Tracer

56 A Switchable Polarization Slot Antenna with DGS For Wlan/Hiperlan Applications

57 A Controllable Band -Notched UWB Antenna with Pin Diodes For On Demand Wi -Max/WLAN/C/X Band Rejection

58 A Novel on Smart Antennas To Improve Performance In Wireless Communications

59 Study of Changes In Antenna Performance When Subjected To DiElectric Filled Metamaterials Over The Frequency Range Upto 50ghz 
63 Performance Analysis of Absolute Deviation Filter For Removal Of

Impulse Noise

64 Large Antenna Array Synthesis Using Modified Differential Evolution

Algorithm

65 Design of Triangular Patch Antenna With Reduced Rcs For Stealth

Applications Using Bionics

66 Location Monitoring System with Image Database

66

67 Control of Home Appliances Using Mobile Phone

67

68

68

Replica Redundancy Block Multiplier Using Fixed Width

69

69 Dynamic Comparators an Overview Ieee 802.16)

71 An Image Encryption and Decryption Using Chaos Algorithm

72 Review on Advancement of TDD LTE Communication Network And

Beyond

73 Implementation of IoT Based Smart Surveilliance Monitoring and Controlling System

74 QCA Design and Performance Analysis Of Binary To Excess Code Converter

75 Validation of Rain Attenuation Time Series Synthesizers To Predict The Artificial Rain Attenuation For Propagation Impairments In Satellite Communications

76 A Novel Low-Power 5th Order Analog to Digital Converter For Biomedical Applications

$77 \quad$ Pv Variations of Pulsed Latch Circuits

78 Design of Multiband Microstrip Patch Antenna For WLAN, Wimax And X-Band Applications

79 Metamaterial Loaded CSRR Based Antenna for WLAN And IoT Band Applications

80 An Implementation of Futuristic Deep Learning Neural Network For Neurons Recognition In Proteomic And Genomic Payload Images

81 Volcano Monitoring System Using Drone-Bot and Iot

82 Structural and Optical Properties Of Fe Doped Tio2nanoparticles For Efficient Photocatalytic Applications

83 Metal and Non-Metal Doped Tio2 Nanoparticles For Enhanced Photovoltaic Device Applications

84 Inner-Gate-Engineered GAA MOSFET To Enhance the Electrostatic Integrity

85 Performance Analysis of Gaa Mosfet For Lower Technology Nodes

86 An Optimal Hybrid Solution to Local And Global Facial Recognition Through Machine Learning

87 Data Security in Cloud Computing Using Three-Factor Authentication

88 A Novel Approach in Optimal Parameters Selection For High Performance And Ultra-Low Power $\sum \Delta$ Modulator

89 Systematic Design and Analysis Of 3rd Order Single Loop $\sum \Delta$ Modulator for ECG Signal Processing

90 Effect of Moderate Atomic Mass Ions On INAS/GAAS Quantum Dots 
93 Approximate Sum-of-Products Design Based on Quantum-Dot Cellular

Automata Using Distributed Arithmetic

94 Transition Analysis of Beam Scintillation In R-Fsoc Links Using Discrete Markov Chain Model

95 Bio-Sensing wwith Enhanced Sensitivity by Noise Spectroscopy Analysis

95

96 A Low Power Auto Adaption Unit for Reconfigurable Data Converter Design of Low Power, Low Voltage Single-Phase Clocking Flip-Flops for Sequential Circuits

98 A Compact Triple Notch Band Cpw-Fed Flexible Antenna for UWB Applications

99 A Liquid Crystal Polymer Substrate-Based Frequency Reconfigurable Monopole Antenna for Vehicular Communication Applications

100 Cantilever Based Mems Sensor for Agricultural Applications

101 Design and Analysis of Rf Mems Switch for Wireless Communications

102 Wind Energy Assessment Over Andhra Pradesh Using High Resolution

103 Time-Frequency Analysis for Enhanced Subsurface Detail Visualization in Non-Stationary Thermal Wave Imaging

104 Pulse Compression Using Fractional Fourier Transform for Enhanced Depth Resolution in Quadratic Frequency Modulated Thermal Wave Imaging

105 Design of Ternary D-Latch Using Graphene Nanoribbon Field Effect Transistor

106 Design and Implementation of Advanced 64-Bit Pipelined Risc Processor for Several Applications

107 Cryptography AES Algorithm Power Calculation Using Clock Gating Technique

108 Design and Fabrication of Saw Resonators On Silicon Substrate Employing Transverse Baw In Patterned ALN

109 Modelling and Simulation of Edge Reflection Type Saw Ladder Filters On Silicon Substrate Using Zno Films

110 IoT Based Global Navigation Satellite System (Gnss) Ground Monitoring System Using Cloud

111 Velocity Synchronous Linear Chirplet Transform Based Subsurface Analysis in Non-Stationary Thermal Wave Imaging

112 An Experimental Investigation of Correlation Based Matched Filter Approach Using Barker Coded Thermal Wave Imaging

113 Underwater Target Tracking Using Fusion of Bearings

114 Passive Target Tracking Using Fusion of State Vectors From Two Sensor Arrays

115 Detection of Dengue Disease by Using Mems Biosensor

116 Capacitance Based Piezoelectric Energy Harvester 116

117 Design of AES Based Cryptography System Using Verilog Hdl 117

118 Implementation of Optimized Radix 4 Booth Algorithm 118

119 FPGA Design of AES Based Chiper And Decipher Cryptography System 119

120 FPGA Implementation of Optimized Radix 4 And Radix 8 Booth 120 Algorithm 
121 Design and Analysis of Coax-Fed U-Slot Antenna for Wireless

Applications

122 Multiband Micro Strip Antenna Design for Space Research Applications

122

123 Design of Nano Scale Square Ring Resonator Band-Pass Filter Using

Metal-Insulator-Metal

124 Performance Analysis of Micro-Cantilever Shape Bio-Sensor for Swine Flu Detection

125 A Robust IoT Based Global Navigation Satellite System (Gnss) Ground Monitoring System Using Cloud

126 Modelling and Prediction of Covid-19 For Tamil Nadu, India

127 A System of Beholding Patient and Hospital Management Using Rfid 127

128 An Optimal Hybrid Solution to Local and Global Facial Recognition 128 Through Machine Learning

129 Data Security in Cloud Computing Using Three-Factor Authentication

130 Vigorous Image Transmission Using Turbo Codes 130

131 Authentication Process in Smart Card Using Sha-256 131

132 Mining Environment Monitoring Based on Laser Communication with 132 Internet of Things

133 Investigation on Cluster Based Application Mapping Approach For Power Optimization In NOC

134 Modified Bat Algorithm for Cluster Based Application Mapping Method 134 In $2 \mathrm{~d} \mathrm{Noc}$

135 Design and Simulation of All-Optical Xor Gate and Its Implementation in Binary to Gray Code \& Gray To Binary Code Converter Using Semiconductor Optical Amplifier

136 Ultrafast All Optical Parity Generator and Checker Based on Quantum Dot Semiconductor Optical Amplifier

137 Design and Implementation of High Entropy TRNG Using FPGA 137

138 Hand-Drawn Circuit Recognition with Deep Learning 138

139 FPGA Implementation of Efficient PCA Technique 139

140 Detection of Objects for Autonomous Cars Using Lane Detection Method 140

141 Design of Birds Detector and Repellent Using Frequency Based Repeller 141 By Machine Learning Algorithm

142 Transition Analysis of Beam Scintillation In R-FSoC Links Using 142 Discrete Markov Chain Model

143 Analytical Tracking of Beam Fluctuations In Random Media 143

144 Design of Nanoscale Square Ring Resonator BPF Using MIM Waveguide 144

145 ARMA Model for Short Term Tec Prediction 145

146 Forecasting of GPS Ionospheric Time Delays Using Fuzzy Inference 146 Systems

147 Analysis of 6 transistor Full Adder 147

148 Analysis and Application of Python on Machine Learning 148

149 Roac: Recursive Optimization of Ant Colony Assisted Perturb and 149 Observe for A Photo Voltaic Resonant Boost Converter

150 Design and Optimization of Single Photon Indium Phosphide on Silicon $\quad 150$ Chip for FMCW Lidar

151 Implementation of Transform Coding Through C Based Vivado Hls 151

152 High Performance 2d Transform Using Area Efficient Methodology for $\quad 152$ Future Video Coding

153 Analysis of An Energy-Balanced Underwater Acoustic Sensor Networks 153 
154 Implementation of Real Time Moving Object Detection Using Region-

Based Fully CNN

155 An Efficient Compression for ECG Signal Using Spiht Algorithm And

Classification Using Fuzzy Svm System

156 Efficient Estimation and Removal of Noise Parameters From Current

Read Out Digital Image Sensor Using Variance Transforms

157 Design of Nanoscale Square Ring Resonator BPF Using Mim Waveguide

158 Reduction of Peak to Average Power Ratio in OFDM System By Using

Selected Mapping Technique

159 Transition Analysis of Beam Scintillation In R-Fsoc Links Using Discrete Markov Chain Model

160 Analytical Tracking of Beam Fluctuations in Random Media

160

161 Detection of Objects for Autonomous Cars Using Lane Detection Method

161

162 Design of Birds Detector and Repellent Using Frequency Based Repeller

162 by Machine Learning Algorithm

163 A Novel Method to Detect Osa Using Deep Convolution Neural Network

164 Analytics Models for Diabetes Prediction Using A Machine Learning 164

165 An IoT Based Multiform Cooling System with Security 165

166 Hand-Drawn Circuit Recognition with Deep Learning 166

167 Audio Denoising by Time-Frequency Block Thresholding 167

168 Automatic Speech Recognition in Meeting Rooms 168

169 Effective Microstrip Feed Line Length for Ultra-Wide Band Responses 169 and Wireless Applications

170 Distributed Peer-To-Peer Data Storage and Synchronizing Websites 170

171 Brain Tumor Detection from Mri Images Using Optimization 171 Segmentation Techniques

172 IoT Based Reconfigurable Smart City Architecture $\quad 172$

173 Improvement of Image Reconstruction Using Multiscale Am-Fm Methods 173

174 Performance Analysis of Absolute Deviation Filter For Removal of 174

175 An Investigation on The Impact of Machine Learning in Wireless Sensor 175

Networks and Its Application Specific Challenges

176 Nuclear Structure of Some Even and Odd Nuclei Using Shell Model 176

Calculations

177 Fuzzy Utilization in Speech Recognition and Its Different Application 177

178 Emotion Recognition Using Knn 178

179 Design and Analysis of Split Ring Antenna with Frequency 179

Reconfigurability Lte And Wimax Applications

180 Estimation of Statistical Parameters Using Image Analysis in Pure and 180

181 Design and Analysis of a Uniform Meander Rf Mems Switch 181

182 Synthesis of Paa For SLL Reduction Using the Differential Evolution 182

Algorithm

183 Mathematical Modeling Approaches for Block Chain Technology 183

184 An Investigation on The Impact of Machine Learning in Wireless Sensor $\quad 184$

Networks And Its Application Specific Challenges

185 Design of Two Stage CMOS Comparator with Improved Accuracy in 185

Terms of Different Parameters

186 Two Stage CMOS Operational Amplifier: Analysis and Design 186

187 FPGA Implementation of Efficient PCA Technique 187 
188 FPGA Implementation of Cad Technique

189 Smart Particle Swarm Optimization (Spso) And Its Application in Image

Compression

190 Chaotic Smart Particle Swarm Optimization (Spso)

190

191 Wind Driven Algorithm-Based Optimization for Synthesis Of Antenna

192 Planar Antenna Array Synthesis Using Cat Swarm Optimization

193 Synthesised Copper Nanoparticles Incorporated in Polyvinyl Alcohol

Nanocomposite for Food Applications

194 Design of Reversible Binary to Gray and Gray to Binary Converter Using Qca

195 IoT Based Reconfigurable Smart City Architecture

196 Brain Tumor Detection from MRI Images Using Optimization

Segmentation Techniques

197 Effective Microstrip Feed Line Length for Ultra-Wide Band Responses and Wireless Applications

198 Audio Denoising by Time-Frequency Block Thresholding

199 Automatic Speech Recognition in Meeting Rooms

200 Distributed Peer-To-Peer Data Storage and Synchronizing Websites

201 Design and Analysis of Reconfigurable Antenna for On-Body Applications

202 Circularly Polarized Ring Antenna with Spokes for Ism Band Applications

203 A Modified Hybrid Planar Antenna for Cognitive Radio Application 203

204 An Ultra-Wide Band Octagonal Antenna with Reconfigurable 204 Narrowband Antenna For Cognitive Radio Applications

205 Ai Based Programmed Imperfection Location Using Thermal Wave Imaging

206 Anomalies Detection in Quadratic Frequency Modulated Thermal Wave Imaging Using Fuzzy C-Means Clustering

207 Design Analysis and Fabrication of FINFET Using 3nm Technology

208 Investigation on Cluster Based Application Mapping Approach for Power 208 Optimization In NOC

209 Modified Bat Algorithm for Cluster Based Application Mapping Method In $2 \mathrm{~d}$ NOC

210 Detection of Objects for Autonomous Cars Using Lane Detection Method

211 Design of Birds Detector and Repellent Using Frequency Based Repeller By Machine Learning Algorithm

212 A Novel Approach in Optimal Parameters Selection for High

Performance and Ultra-Low Power $\sum \Delta$ Modulator

213 Systematic Design and Analysis Of 3rd Order Single Loop $\sum \Delta$ Modulator 213 for ECG Signal Processing

214 An Optimal Hybrid Solution to Local and Global Facial Recognition 214 Through Machine Learning

215 Data Security in Cloud Computing Using Three-Factor Authentication 
219 Fabric Substrate Material Based Multiband Spike Antenna for Wearable

Applications

220 Pentagon Shaped Microstrip Antenna for Wireless IoT Applications

220

221 Transmission Line Effects and Cross Talk Effets On Interconnects in

Deep Submicron Vlsi

222 Epilepsy Seizure Alert System Using IoT

223 Synthesised Copper Nanoparticles Incorporated in Polyvinyl Alcohol

Nanocomposite for Food Applications

224 Design of Reversible Binary to Gray and Gray to Binary Converter Using QCA

225 Reduction of Peak to Average Power Ratio in OFDM System by Using

Selected Mapping Technique

226 Evaluation of Adverse Effects of Fog on Fso Links

227 Understanding the Effects of Various Modulation Techniques on Fso Links

228 A Novel Low-Power 5th Order Analog to Digital Converter For Biomedical Applications

229 PV Variations of Pulsed Latch Circuits

230 Design of Magnitude Comparator Using Adiabatic Logic in Ecrl

231 Design of Xor And Xnor Gates Based on Full Adder Circuits

232 QCA Design and Performance Analysis of Binary to Excess Code

233 Validation of Rain Attenuation Time Series Synthesizers to Predict The

Artificial Rain Attenuation For Propagation Impairments In Satellite Communications

234 Design and Implementation of Full Adder Using Interconnects for Low Power and High-Speed Application

235 Direction of Arrival Estimation Using Conventional Subspace Algorithms

238 Efficient ECG Signal Compression and Transmission Algorithm Using Dual Encoding Technique

239 Smoothing and Optimal Compression of Encrypted Gray Scale Images

242 Design of Magnitude Comparator Using Adiabatic Logic in Ecrl

243 Artifact Elimination in Thoracic Electrical Bioimpedance Signals Using

244 Energy Detection for Spectrum Sensing in Medical Telemetry Networks Using Modified Nlms Algorithm

245 Design of Xor And Xnor Gates Based on Full Adder Circuits

248 Implementation Of C-Element And D-Element Edge Triggered Flip Flop 248 for Vlsi Applications

249 Design Alu Based Online Bist For Multi Word-Width Ram's 249

250 A Novel Approach in Optimal Parameters Selection for High 250 Performance and Ultra-Low Power $\sum \Delta$ Modulator

251 Systematic Design and Analysis Of $3^{\text {rd }}$ Order Single Loop $\sum \Delta$ Modulator for Ecg Signal Processing 
252 Appearance Invariance Action Recognition with Multi Stream Convnets

On Unconstraint Indian Classical Dance Videos

253 A Spatial Two Stream Cnns For Human Action Recognition from Real

Time Video Sequences

254 Speaker Identification in Variable Health Conditions

254

255 Design of A Fault Tolerant Adder for Delay Reduction in Fir Filter 255

256 A Novel Method of Using Auxiliary Information for Compressing 256 Encrypted Images

257 An Effective Face Recognition Method Using Gabor Wavelet Transform

258 Design of Nanoscale Square Ring Resonator Plasmonic Filter Using Mim For Dual Band Applications

259 Concurrent Dual and Triple Band Square Ring Resonator Base-Band Filter Using MIM For Plasmonic Applications

260 An Ultra-Wideband Band Pass Filter Using Mim Waveguide for Nano Scale Applications

261 MIM Based Stepped Impedance Square Ring Resonator Dual-Band Band Pass Filter

262 Analysis of Power Spectrum Density on Earthquake Data Using Modified Covariance Algorithm

263 Power Spectrum Estimation of Seismic Wave Using Periodogram Method

264 Spectral Analysis of Seismic Signals Using Burg Algorithm

265 Underwater Target Tracking System Using Active Sonobuoys

266 Quad-Band Ring Loaded Circular Patch Antenna with Meander Line Slot for Wireless Applications

267 Dual Annular Ring Coupled Stacked Psi Shape Patch Antenna for Wireless Applications

268 CPW Fed Compact Overlapped Annulus Patch Antenna with Incorporated Psi Shapes for Ism Band and WLAN Applications

269 Circular Monopole Patch Antenna Design with CSRR DGS for Wireless Applications

270 Machine Learning Techniques for Automatic Defect Detection in Frequency Coded Thermal Wave Imaging

271 Clustering in Non-Linear Thermal Wave Imaging for Defect Detection

272 Orthogonal Projection for Enhanced Subsurface Detection in NonStationary Thermal Wave Imaging

273 Composite Inspection Through Quadratic Frequency Modulated Thermal Wave Imaging with Spectral Reshaping

274 Design of A Non- Uniform Serpentine Asymmetric Cantilever Rf- Mems Shunt Capacitive Switch for Radar Applications

275 Electromagnetic Analysis of Mems-Based Tunable EBG Band stop Filter Using Rf Mems Switch for Ku-Band Applications

276 Design and Simulation of Mems Based Capacitive Pressure Sensor for Harsh Environment

277 Design and Performance Analysis of Hybrid SPDT Rf Mems Switch

278 Design and Performance Analysis of Double Cantilever Type Capacitive Shunt Rf Mems Switch

279 A Modified Proposed Capacitance Model for Step Structure Capacitive Rf Mems Switch by Incorporating Fringing Field Effects

280 Precipitation and Other Propagation Weaknesses Impacts at Microwave and Milli meter Wave Groups: A Scaled Down Study 
281 Combination of Phased Array Antenna for Side Flap Level Decrease

Utilizing the Differential Development Algorithm

282 Plan and Analysis of Capacitive Shunt Rf Mems Switch for

Reconfigurable Antenna

283 Hybrid Beam Directing of Ka-Band Array-Fed Reflector Antenna for

Satellite Communication Links

284 Design and Analysis of Circular Monopole Antenna for Wireless

Applications

285 Enhancement of Gain Using Hybrid Reconfigurable Trapezoidal Antenna with FSS

286 Design and Analysis of CPW-FED Octagon Shaped Ring Slot Monopole Antenna

287 A Triple Band Flower Shaped Microstrip Antenna with Circular Ring Loading

288 A Concentric Ring Heart Shaped Monopole Antenna for WIMAX Wireless Applications

289 Raspberry Pi Based Personal Assistance and Artificial Intelligence (AI) Disabled People

290 Modification of Parameters of the ITU-R to Enhance the Estimation of Tropospheric Scintillation for Tropical Areas

291 Estimation of Propagation Impairments and Melting Layer in The

Southern Part of Indian Subcontinent

292 Design \& Analysis of Multi-Layer Edge Feed Stacked Patch Antenna For S-Band Applications

293 Rain Rate-Radar Reflectivity Relationship for Drop Size Distribution and Rain Attenuation Calculation of Ku Band Signals

294 Design of Uhf Antenna for Wireless Applications Using Defective Ground

295 Estimation of Effect of Troposphere Rain on Radio Link in Tropical Environment

$2962 \times 2$ Microstrip Patch Antenna Using Corporate Feed

297 Design \& Analysis of Electrically Small Top Loaded Antennas for GPS Applications

298 Analysis of Small Antenna with Slotted Meander-Line Resonator for Wireless Applications

299 Analysis of Microstrip Circular Patch Antenna Array

300 Non-Destructive Characterization of CFRP Composite Using Non-

301 Black-Hole Attacks in AODV Routing Protocol for Mobile Ad-Hoc Networks

302 Performance Metrics Between Routing Protocols for Manets

303 Visualization of Performance Metrics Between Routing Protocols for Manets

304 Efficient Cell Sizing of Single Precision Floating Point ALU For DSP Applications

305 Implementation of Environment Gases Monitoring System Using LORA Gateway in Smart Cities with IoT Technology

306 An Embedded Web Server Based Automatic Smart Home Monitoring System

307 Lora System Based Accident Rescue System for Future Generation 
308 A Vehicle-To-Vehicle Communication Using Can Network and Zigbee

Technology

309 Dm3730 Processor Hardware Debugging on Linux Platform

310 Hardware Prototyping for Video and Signal Processing Applications 310

Using Dm 6437 Texas Instrument Evaluation Board

311 Optimization of Smart Vehicle Ad Hoc Network (SVANET)

Communication for Traffic Related Issues with A Security

312 Hardware Implementation of TLBO Algorithm for Cognitive Radio

Networks

313 Cloud Enabled Neural Network with Intelligent Sensor Nodes for HVAC

314 Energy Monitoring Using ARM 7

315 Smart Vehicle Tracking System Using RFID 315

316 IoT Based Forest Fire Detection System 316

317 Big Data Analytics and IoT Gadgets for Tech Savvy Cities 317

318 Performance Analysis of Layered Architecture to Integrate Mobile 318

Devices and Grid Computing with A Resource Scheduling Algorithm

319 Securing SAAS Service Under Cloud Computing Based Multi-Tenancy Systems

320 A Transfer Learning Framework for Traffic Video Using Neuro-Fuzzy Approach

321 An IoT Based Reference Architecture for Smart Water Management

Processes

322 Emergency Vehicles Pre-Emption

323 Smart Home Automation Security

324 Improving the Verification of Ontology Authoring Actions 324

325 Non-Linear System Identification Using Kernel Based Exponentially 325

326 Detection and Classification of COVID 19 Using Transfer Learning with 326

327 Classification of Disturbances in A PV Based Distributed Generation

System Using Cross VMD and Random Vector Functional Link Network

328 Real-Time Anomaly Detection Using Tensor Flow Based RNN Deep Learning Classifier

329 Smart Home Automation Using IoT

330 An Approach to Hybridization of Embedded System Networks

331 Access Control of Door Using Face Recognition and Home Security Alert Using Raspberry Pi and Internet

332 Recognition of Vehicle Number Plate and Measure the Distance 332

333 Modeling of GPS-TEC Using QR-Decomposition Over the Low Latitude 333 Sector During Disturbed Geomagnetic Conditions

334 Application of Singular Spectrum Analysis Using Artificial Neural 334 Networks in TEC Predictions for Ionospheric Space Weather

335 Implementation of IoT Analytics Ionospheric Forecasting System Based on Machine Learning and thing speak

336 Ionospheric Monitoring System Based on the Internet of Things with Thing speak

337 Water Leakage Detection Monitoring and Controlling System Using IoT

338 Broadcast- Based Data Gathering Mechanism for Sampling Sensor Fields

339 A Study of Intrusion Detection Systems in Heterogeneous Wireless 
340 Detection of Active Internet Worm: Camouflaging WORM

341 A Study of Intrusion Detection Systems in Heterogeneous Wireless

Sensor Networks

342 Delay Analysis

343 Family Based Efficient Routing Protocol (FERP) For Lifetime Improvement in Heterogeneous Wireless Sensor Networks

344 Green Comp Based Energy Efficient Data Aggregation Algorithm with Malicious Node Identification (Geed-M) For Lifetime Improvement in WSN

345 Analysis of Throughput and Spectrum Handoff Delay in Cognitive Radio

346 Implementation of Distributed Power Control/Active Link Protection for FEMTO Cell Networks

347 Analysis of Different Direction of Arrival (DOA) Estimation Techniques

Using Smart Antenna in Wireless Communications

348 Clock Synchronization in an N-Modular Redundant System

349 Development of Internet of Things Based Decision Support for Vehicle

Drivers by Using GPS and GSM

350 Intrusion Detection System Using Ai And Machine Learning Algorithm

351 Implementation of IoT Based Vehicle Theft Detection and Accident Monitoring System Using Arduino

352 Tokenization of News Feed Articles Based on Their Similarity Using Machine Learning Techniques

353 A Novel Graphical Password Authentication Mechanism for Cloud Services

354 A Graphical Password Authentication System or Touch Screen Based Devices

355 Random Grid Size Based Graphical Password Authentication

356 Defected Ground Structure Switchable Notch Band Antenna for UWB Applications

357 Planar Switchable Notch Band Antenna with DGS for UWB Application

359 Reconfigurable Notch Band Antenna Using Pin Diodes 



\title{
Design of elliptical planar monopole antenna for UWB applications
}

\author{
M.Venkata Narayana ${ }^{1}$, Govardhani.Immadi ${ }^{2}$ \\ ${ }^{1,2}$ Department of ECE, Koneru Lakshmaiah Education Foundation, \\ Green Fields, Vaddeswaram, A.P., India-522502. \\ E-mail: mvn@kluniversity.in.
}

\begin{abstract}
:
Here, an elliptical planar monopole Penta band-notched ultra-wideband (UWB) antenna is designed. Band rejection at 2.4-2.6 GHz IEEE 802.11 b/g/n, 3.3-3.75 Wi-MAX, 3.9-4.2 GHz Cband satellite communication, 5.15-5.85 WLAN, and 7.9-8.4 GHz X-band satellite communication frequencies is accomplished by etching slots in the radiating patch, feed line, and ground plane. The proposed antenna was fabricated and tested. The measured impedance bandwidth of the antenna is $2.15-12.5 \mathrm{GHz}$, which covers Bluetooth and UWB applications. The peak gain of the proposed antenna is around $8 \mathrm{~dB}$ and drops drastically at notched bands. The antenna shows good omnidirectional radiation patterns in the passbands.
\end{abstract}

Index Terms - Monopole, notch bands, patch, satellite communication, WLAN, antenna 


\title{
A Multilayer antenna with stacked structure for ISM Applications
}

\author{
M.Venkata Narayana ${ }^{1}$, Govardhani.Immadi ${ }^{2}$ \\ ${ }^{1}$ Department of ECE, Koneru Lakshmaiah Education Foundation, \\ Green Fields, Vaddeswaram, A.P., India-522502. \\ E-mail: mvn@kluniversity.in.
}

\begin{abstract}
:
A new multilayer stacked substrates $\mathrm{H}$-shaped patch antenna with microstrip feedline is proposed. The designed structure has stacked FR-4 substrates with embedded ground plane. To accomplish the ISM $(2.4 \mathrm{GHz})$, the configured $\mathrm{H}$-shaped patch antenna with an opening slot on ground and the novel $\mathrm{H}$-shaped patch antenna imposed over a second layer. The results demonstrate that return loss is $-26.6 \mathrm{~dB}$ and VSWR 1.09 at $2.4 \mathrm{GHz}$. An intended multilayer antenna has $5.6 \mathrm{dBi}$ gain at $2.4 \mathrm{GHz}$. The optimized structure is easy to acquire, has less volume, compact size, and adequate bandwidth of deployment. For ISM band applications, the configured multilayer patch antenna is appropriate. Here, the results revealed that it is indeed a great longeffective range antenna performance for WLAN correspondence on the working band in exercise. Reenactment is finished by utilizing computer simulation technology (CST).
\end{abstract}

Index Terms - patch,Monopole, notch bands, satellite communication, WLAN, antenna 


\title{
Detailed analysis on the atmospheric effects on FSO links at $405 \mathrm{~nm}$ Govardhni.Immadi ${ }^{1}$,A.Sree Madhuri ${ }^{2}$,M.Venkata Narayana ${ }^{3}$ \\ ${ }^{1,2,3}$ Department of ECE, Koneru Lakshmaiah Education Foundation, Green Fields, Vaddeswaram, A.P., India-522502. \\ E-mail: govardhanee_ec@kluniversity.in.
}

\begin{abstract}
:
Free- space optics (FSO) is a LOS communication which is earning a lot of recognition in the current research as it offers many advantages such as license free communication, high bandwidth so on. Like the other technologies, FSO also undergoes from adverse effects due to the climatic changes in the atmosphere. Some of the prominent effects that degrade the strength of the signal in free space are the fog, snow, haze, and rain. In this paper, the effects of fog and haze on the FSO link were investigated with the support of both simulated and experimental results. An experimental setup containing FSO system enclosed in a glass cabinet is used, and the simulation is carried out in OptiSystem prediction models. Results demonstrate that as the intensity fog or the intensity of haze increases the received voltage levels decline.
\end{abstract}

Index Terms - Haze, Fog, link, communication, experimental setup, attenuation. 


\title{
Design of Linear Rectangular Microstrip Antenna for Ka band Applications
}

\author{
Govardhni.Immadi ${ }^{1}$,M.Venkata Narayana ${ }^{2}$ \\ ${ }^{1,2}$ Department of ECE, Koneru Lakshmaiah Education Foundation, \\ Green Fields, Vaddeswaram, A.P., India-522502. \\ E-mail: govardhanee_ec@kluniversity.in.
}

\begin{abstract}
:
The demand for high data transmission with nonstop service leads to usage of very high frequency. To meet the demands ISRO launched GSAT-14 with two Ka-Band BECONS at $20.2 \mathrm{GHz}$ and $30.5 \mathrm{GHz}$. Via this design and analysis of one, two $(1 \times 2)$, four $(1 \times 4)$ and eight $(2 \times 4)$ element linear rectangular shaped microstrip array antennae at a frequency of $20.2 \mathrm{GHz}$ is accomplished to attain high frequency access, high gain and directivity. The substrate material used is identical for the one, two $(1 \times 2)$, four $(1 \times 4)$ and eight $(2 \times 4)$ element i.e. "Rogers RT/Duroid 5880" with thickness and Relative permittivity of $0.254 \mathrm{~mm}$ and $2.2 \mathrm{~mm}$ is considered. The antenna elements placed linearly with equal distance and the feed network applied here is a corporate or parallel feed network. The RF Wilkinson power divider is used for impedance matching and to excite the number of elements (n) from a single source to drive all elements equally with loss less resistive network. The proposed array antennae are simulated using HFSS software and fabricated observations are analyzed.
\end{abstract}

Index Terms-network, array, antenna, elements, transmission, frequency 


\title{
A Novel Method to Detect OSA Using Deep Convolution Neural Network
}

\author{
Sanam Nagendram ${ }^{1}$, G. Naga Surya Prakash', N. Pavan Srinivas ${ }^{3}$, B. \\ Raja V R Chowdary ${ }^{4}$, \\ ${ }^{1}$ Associate Professor, Department of ECE, Koneru Lakshmaiah Education Foundation, \\ Vaddeswaram, A.P., India-522502. E-mail ID: nagendram@kluniversity.in \\ ${ }^{2,3,4}$ U.G Students, Department of ECE, Koneru Lakshmaiah Education Foundation, \\ Vaddeswaram, A.P., India-522502.
}

\begin{abstract}
:
Obstructive sleep apnea (OSA) is a general problem where individuals breathing are disturbed in the sleep. Major sleep apnea issues are presently unidentified because of cost and real time limitations of overnight poly so mnography (PSG) at sleep experiments, where an expert professional monitoring is required to work over night. Recent techniques for sleep apnea classification are being designed by bioengineers for most feasible and accurate detection. In this paper, a classification algorithm automatically processes short intervals epochs of the electrocardiogram (ECG) input. Using support vector machines (SVM), the classification algorithm automatically trains and tests the data on subjects with and without OSA. One of the new approaches for this study is obtaining the information from single channel ECG recordings for the detection of OSA. The present work contributes the development of diagnosing the Sleep Apnea based on Machine Learning algorithms. This ML based detection helps in consuming less time and with the usage of this technique helps in improving its efficiency.
\end{abstract}

INDEX TERMS: Obstructive sleep apnea, electrocardiogram, and epochs. 


\title{
Analytics models for Diabetes Prediction using a Machine Learning
}

\author{
Sanam Nagendram ${ }^{1}$, R Vijay Kumar Reddy ${ }^{2}$, G Jagadish ${ }^{3}$ \\ ${ }^{1}$ Associate Professor, Department of ECE, Koneru Lakshmaiah Education Foundation, \\ Vaddeswaram, A.P., India-522502. E-mail ID: nagendram@kluniversity.in \\ ${ }^{2,3}$ U.G Students, Department of ECE, Koneru Lakshmaiah Education Foundation, \\ Vaddeswaram, A.P., India-522502.
}

\begin{abstract}
:
Diabetes is one of the essential illnesses everywhere at some degree inside the area. Consistent with the International Diabetes Federation (IDF) document of the three hundred and sixty five days 2017, diabetes isn't abnormal in greater or less eight.8\% of the Indian person human beings and is one of the incredible ten perception techniques in destruction in India. In untreated and unidentified diabetes should reason adjustments within the sugar tiers and silly occurrences, damage organs, as an instance, kidneys, eyes, and veins in the coronary heart. Via using device gaining knowledge of estimations to anticipate the infection from the applicable datasets at a beginning time need to extra then in all likelihood extra human lives.
\end{abstract}

INDEX TERMS: perception techniques, coronary, IDE. 


\title{
Application of advanced statistical signal processing algorithms for prognostic studies on Earthquakes using Ultra Low Frequency geomagnetic signals
}

\author{
${ }^{1}$ K.S. Ramesh, ${ }^{2}$ N. Swaroop Kumar \\ Department of ECE, Koneru Lakshmaiah Education Foundation, Vaddeswaram, Guntur, A.P, \\ India-522502 \\ Email: swaroopa7@gmail.com
}

\begin{abstract}
:
Earthquake precursors have no definite prognostic values till now. Owing to a lack of statistical reliability analysis of precursors and their validity of their assessment against natural time variability and background variations, the problem of decisive detection and precise prediction of earthquake remain unsolved. Ultra Low Frequency (ULF) being a promising candidate for earthquake precursors because of its larger skin depth. The inherent stochastic nature of ULF. The proposed project is envisaged, to investigate the reliability of earthquake precursors in ULF geomagnetic anomalies using advanced statistical signal processing algorithms. The observed ULF data from different stations is initially subjected to pre-processing through removal of outliers, filling the missing data and conversion of non Gaussian noise into Gaussian noise using Unscented Kalman Filter or Particle (Monte Carlo) Filter. Further, Sliding time window process is carried out by Normalized Squared Innovation Process (NSIP) for detection of abnormalities in ULF data. As this process is recursive, a decision will be made using Chi-squared distribution. Ultimately, the above procedure is useful in designing and deployment of early warning systems to detect earthquake precursors in ULF geomagnetic anomalies for precise forecasting of earthquakes. To Investigate the existence of Ultra Low frequency (ULF) signals around $0.01 \mathrm{~Hz}$ in geomagnetic anomalies associated with Earthquakes. To implement the advanced statistical signal processing algorithms on ULF geomagnetic signals for isolation of earthquake precursors. To identify possible precursors for prognostic study of earthquake facilitating to develop early warning systems.
\end{abstract}

Keywords: Earthquake Precursors, Ultra Low Frequency, Statistical Signal Processing Algorithms 


\title{
of physical phenomenon of seismo-ionospheric perturbations using GPSTEC - a new perspective
}

\author{
K.S. Ramesh ${ }^{1}$, N. Swaroop Kumar ${ }^{2}$ \\ ${ }^{1}$ Professor, Department of Electronics and Communication Engineering, Koneru Lakshmaiah \\ Education Foundation, Vaddeswaram, Guntur, A.P, India-522502.dr.ramesh@kluniversity.in \\ ${ }^{2}$ Research scholar, Department of Electronics and Communication Engineering, Koneru \\ Lakshmaiah Education Foundation, Vaddeswaram, Guntur, A.P, India-522502 \\ Email: swaroopa7@gmail.com
}

\begin{abstract}
:
Earthquakes are one of the natural disasters and are inevitably having adverse effects on human life and his assets. Their detection and prediction mainly depend on understanding the underlying phenomenon, validity of the data acquired at the time of their occurrence through different techniques and development of mathematical models with sophisticated algorithms embedded for signal processing. Efforts are made in this direction to apply sophisticated algorithms such as nonparametric, parametric frequency estimation methods and complex wavelets to discriminate earthquake signatures in GPS TEC from other space weather disturbances.
\end{abstract}

Keywords: Earthquake, GPSTEC, Sophisticated algorithms 


\title{
Development of Blink Restoration model for Facial Paralysis detection
}

\author{
${ }^{1}$ Kalivaraprasad.B , ${ }^{2}$ MVD.Prasad , ${ }^{3}$ Harshavardhan.L. \\ ${ }^{123}$ Department of ECE, Koneru Lakshmaiah Education Foundation, \\ Green Fields, Vaddeswaram, A.P., India-522502. \\ E-mail:baditakali@gmail.com
}

\begin{abstract}
:
On account of blink feature, patients suffer from facial paralysis that lead to disfigurement and loss of vision. Ultimately, facial pacing aims to improve the standard of life of individuals suffering from facial unilateral paralysis. A device is designed that can analyze electromyography signals from the healthy side of the face eye and trigger the corresponding muscle with electrical stimulation on the paralysed side simultaneously. FES is one of the possible ways for restoration of blink of these patients. The system modelled will achieve the EMG signal extraction and blink detection algorithm for stimulation by real time signals. Finally, the device has been experimented on humans, rabbits, dogs with EMG signal data set. The attenuation of power frequency is about $51 \mathrm{~dB}$ with $80 \%$ accuracy.
\end{abstract}

Index Terms: Electromyography, RISC, CMOS Process, Blink Detection 


\title{
Comparative Analysis of Watermarking Methods on CFRP Sample Thermal Images
}

\author{
Kalivaraprasad $^{1}$. B, MVD.Prasad ${ }^{2}$, Rajesh Babu. K ${ }^{3}$ \\ ,SyedShameem ${ }^{4}$,S.Mohan5 Vasudevan.Vani6 \\ ${ }^{1234}$ Department of ECE, Koneru Lakshmaiah Education Foundation, \\ Green Fields, Vaddeswaram, A.P., India-522502. \\ ${ }^{5,6}$ Department of SCIMS, university of South Pacific. Fiji \\ E-mail:baditakali@gmail.com
}

\begin{abstract}
:
Digital watermarking became indispensable in today's scenarios and a lot of digital data have become distributed worldwide.Internet connectivity has made it very convenient and inexpensive. Due to rapid technological progress in the past 15 years, current users There has been a rise in the likelihood of various digital attacks, such as forgery, damaging digital data, downloading, copying and altering digital knowledge. The use of a privacy mechanism that can protect digital data from the owner of digital data and its rights has been relevant in these situations. In this paper, comparing the watermarking techniques such as least significant bit (LSB), DWT-SVD-hybrid (discrete wavelet transform-singular value decomposition) and reversible blind watermarking techniques on thermal images of CFRP (carbon fiber reinforced polymer) sample. The reversible bind watermarking is better for recovering the layout information of the CFRP sample than the LSB and hybrid method. The watermarking strategies of thermal images layout can become more secure.
\end{abstract}

Index Terms: Steganography, Watermarking, DWT, SVD, CFRP sample. 


\title{
Genomic Analysis using Higher Order Adaptive Exon Predictors
}

\author{
Nagesh Mantravadi ${ }^{1}$, Md Zia Ur Rahman ${ }^{2}$ \\ 1,2 Department of ECE, Koneru Lakshmaiah Education Foundation, \\ Green Fields, Vaddeswaram, A.P., India-522502. \\ E-mail: ${ }^{1}$ nageshsarma@kluniversity.in, ${ }^{2}$ mdzr55@gmail.com
}

\begin{abstract}
:
In genomics, true identifying exon regions in deoxyribonucleic acid (DNA) sections are an important activity for the identification and development of disease medications. All exon identification techniques are based on three basic periodicity (TBP) properties of exons. The techniques of adaptive sign processing have been successful compared to various other methods. This paper uses the least mean fourth (LMF) algorithm also its signed variants that includes SRLMF, SLMF also SSLMF algorithms to develop multiple adaptive exon predictors (AEPs) with less computational complexity. Eventually, a performance evaluation is performed for different AEPs using various standard gene data sequences derived from National Biotechnology Information Centre (NBI) genomic sequence database, such as Sensitivity (Sn), Precision (Pr) and Specificity (Sp) measurements.
\end{abstract}

Index terms - adaptive exon predictor, computational complexity, deoxyribonucleic acid, disease medications, exon, three base periodicity 


\title{
Efficient Cardiac Signal Enhancement Techniques Based on Variable Step Size and Data Normalized Hybrid Signed Adaptive Algorithms
}

\author{
Nagesh Mantravadi ${ }^{1}$, Md Zia Ur Rahman ${ }^{2}$ \\ 1,2 Department of ECE, Koneru Lakshmaiah Education Foundation, \\ Green Fields, Vaddeswaram, A.P., India-522502. \\ E-mail: ${ }^{1}$ nageshsarma@kluniversity.in, ${ }^{2}$ mdzr55@gmail.com
}

\begin{abstract}
:
In remote health care monitoring, the extraction of high resolution cardiac signals is an important task. For this the cardiac signal (CS) needed to be enhanced. Among various filtering techniques, the adaptive noise cancellation (ANC) is a promising methodology. In adaptive filtering least mean square (LMS) algorithm is the fundamental enhancement algorithm. However, it suffers with slow convergence and weight drift problem in non-stationery environment. In order to improve the performance of ANC this paper proposes to implement an ANC methodology based on variable step size on the normalization of fundamental LMS algorithm for CS enhancement. Based on such strategy this research implements ANC using hybrid algorithm called variable normalized LMS (VNLMS) algorithm. Further, to improve the convergence characteristics, to filter the ability and to minimize computational complexity some versions of VNLMS algorithms are implemented too. Finally, the proposed ANCs are tested using real CS obtained from MIT-BIH data base. The performance evaluation is carried based on signal to noise ratio improvement (SNRI) and excess mean square error (EMSE).
\end{abstract}

Index terms - Arrhythmia; Artifacts; Convergence; Computational Complexity; Least Mean Square 


\title{
Classification of dress codes using CNN
}

\author{
D.Bhavana ${ }^{1}$, K.Kishore Kumar ${ }^{2}$ \\ ${ }^{1}$ Department of ECE, Koneru Lakshmaiah Education Foundation, Vaddeswaram, AP., India- \\ 522502 \\ ${ }^{2}$ Department of ME, Koneru Lakshmaiah Education Foundation, Vaddeswaram, AP., India- \\ 522502 \\ Email ID: bhavanaece@kluniversity.in
}

\begin{abstract}
:
Individuals now live in a world encompassed by corporate culture like work environments and universities, schools, emergency clinics. Specifically instructive organizations will guarantee the understudies to follow clothing regulation to get consistency among the understudies. It is a dull errand to the administration to distinguish the understudies who doesn't follow the clothing standard. Manual perception requires more human inclusion and it isn't workable for the whole day. More over the grounds is altogether enormous and checking understudies everywhere on the grounds physically expands the remaining burden. To determine those issues, the proposed model presents a neural organization based characterization framework to distinguish and classify the understudies. Informational index comprises of 270 pictures of understudies and experts are utilized in the experimentation to perceive the clothing standard of people. The outcomes gave us exact desires using some graphical depictions similarly as periods of the various people.
\end{abstract}

Keywords: Environments, organizations, perception, neural organization, graphical depictions 


\title{
Random number generator using Labview
}

\author{
D.Bhavana ${ }^{1}$, K.Kishore Kumar ${ }^{2}$ \\ ${ }^{1}$ Department of ECE, Koneru Lakshmaiah Education Foundation, Vaddeswaram, AP., India- \\ 522502 \\ ${ }^{2}$ Department of ME, Koneru Lakshmaiah Education Foundation, Vaddeswaram, AP., India- \\ 522502 \\ Email ID: bhavanaece@kluniversity.in
}

\begin{abstract}
:
Irregular number generator $[\mathrm{RNG}]$ is use to produce arbitrary numbers between some random breaking point, RNG's are two sorts 1. True irregular number and 2.pseudom numbers. Genuine arbitrary numbers are not unsurprising by any numerical equation since they are fundamentally relies upon the climatic commotion, going to the pseudo numbers are predominantly utilized in the vast majority of PCs, this irregularity can be unsurprising by utilizing numerical recipe and it is fine numerous reasons, however it may not be arbitrary in the manner you expect on the off chance that you are utilized to dice rolls and lottery drawings. In this scaled down task we are doing RNG [pseudo numbers] by utilizing NI labview programming and producing arbitrary numbers by squeezing press catch and coming yield we are showing on seven section show. In the labview it simple to produce an irregular number by utilizing distinctive blocks and primary favorable position of the lab view is there is no need of any programming dialects like[c,c++,java, matlab].Main capacity of this undertaking is utilized for gaming and need number age and so on. A grouping of uniform arbitrary numbers, which is produced inside the PC in a deterministic way, is frequently alluded to as a pseudo-irregular number succession.
\end{abstract}

Keywords:Labview, number generator, pseudo numbers, dice rolls, irregular numbers, arbitrary numbers. 


\title{
Energy Detection for Spectrum Sensing in Medical Telemetry Networks using Modified NLMS algorithm
}

\author{
S. Surekha ${ }^{1}$, Md. Zia Ur Rahman ${ }^{1}$, A. Lay-Ekuakille ${ }^{2}$ Antonio Pietrosanto $^{2}$,M. \\ Avoci Ugwiri ${ }^{3}$ \\ ${ }^{1}$ Department of ECE, Koneru Lakshmaiah Education Foundation, \\ Green Fields, Vaddeswaram, A.P., India-522502. \\ ${ }^{2}$ Department of Innovation Engineering, University of Salento, Lecce, Italy \\ ${ }^{3}$ Department of Industrial Engineering, University of Salerno, Fisciano, Italy \\ ${ }^{1}$ E-Mail ID: mdzr55@gmail.com
}

\begin{abstract}
:
In a clinical scenario when the patient is far from the hospital, the patient is not treated in time. In that scenario, the medical telemetry network plays a vital role. So, it is intended to develop a medical body area network for remote health care monitoring utilizing cognitive radio methods. A Medical Body Area Network (MBAN) consists of multiple sensor nodes, these nodes may be wearable and implantable devices and by using this device we get the patient physiological data and each having the capability of sampling, processing and communicating with vital signals. Medical Telemetry is a technology, it involves information processing and communication technologies to utilize in healthcare services. In wireless communications, medical telemetry plays a vital role to use in health care applications like telemedicine, remote patient monitoring, etc., Spectrum sensing is one of the cognitive radio methods, it can be used in the field of medical telemetry. Spectrum sensing using an energy detection technique can be used in medical telemetry for health care monitoring. The proposed implementation finds an immediate application in the development of smart cities and smart hospitals. Here, we use a Modified Normalized LMS algorithm for this purpose. Problems of energy detection and measurement lead to spectrum sensing in a telemetry network that can be solved by the proposed method. Results show that the performance of Modified Normalized LMS gives better simulations in terms of detection probability $\left(\mathrm{P}_{\mathrm{d}}\right)$ and false alarm $\left(\mathrm{P}_{\mathrm{f}}\right)$.
\end{abstract}

Index Terms -Energy detection and measurement, Medical Telemetry, Remote Health Care Monitoring, Spectrum Sensing, Smart hospital. 


\title{
An Efficient optimal threshold-based segmentation and classification model for multi-level spinal cord Injury detection
}

\author{
Sk. Hasane Ahammad ${ }^{1}$, Md. Zia Ur Rahman ${ }^{1}$, A. Lay-Ekuakille ${ }^{2}$, N. I. \\ Giannoccaro \\ ${ }^{1}$ Department of ECE, Koneru Lakshmaiah Education Foundation, \\ Green Fields, Vaddeswaram, A.P., India-522502. \\ ${ }^{2}$ Department of Innovation Engineering, University of Salento, Lecce, Italy \\ ${ }^{1}$ E-mail ID: mdzr55@gmail.com
}

\begin{abstract}
:
Detection of spinal cord injury (SCI) is one of the major problems in children and adults due to variation in shape and orientation. As the types of spinal cord injuries are increasing, it is difficult to find and predict the new type of disorder due to high dimensionality and sparsity problems. Most of the existing models are used to extract either the limited number of features or over segmented features on the SCI data. These models are not applicable to filter the essential features space with less segmented regions for injury disorder prediction. In such a scenario, we propose a hybrid threshold-based image segmentation and classification model is implemented for disorder prediction. In this model, a hybrid Ostu's thresholding method and expectation maximization (EM) approach and robust decision tree classifier are used to filter the essential features for disorder prediction. A hybrid CNN framework is used to extract the feature sets on the segmented features. Finally, a probabilistic classification model is used to predict the disease severity on the segmented image features. Experimental results illustrate the efficiency of proposed disorder prediction model with the existing models with 0.97 accuracy and 0.98 precision rate on the SCI dataset.
\end{abstract}

Index Terms -Boosting technique, Machine learning, spinal cord disc, vertebral column, random forest, segmentation 


\title{
An Early 3D Human Action Recognition (3DHAR) System by Training Sub Actions of an Action using Deep Networks
}

\author{
M. Teja Kiran Kumar ${ }^{1}$, P.V.V. Kishore ${ }^{2}$ \\ ${ }^{12}$ Department of Electronics and Communications Engineering 1 Koneru Lakshmaiah \\ Educational Foundation (KLEF) Deemed to be University Vaddeswaram, Guntur, AP., India \\ Email: mtejakiran@kluniversity.in,pvvkishore@kluniversity.in
}

\begin{abstract}
:
The Human action (HA) comprises of a set continues sub-actions. In this way, our point is to foresee the HA's utilizing those arrangement of sub-actions before finishing an action. Usually, human action recognition (HAR) has been performed utilizing joints, RGB recordings and Depth in all these cases forecasting an incomplete HA became most difficult task. So as to beat this issue, we proposed a novel approach utilizing bidirectional LSTM system so as to characterize the an action from a fully trained convolution neural network $(\mathrm{CNN})$ features. $\mathrm{CNN}$ features are acquired from the 3D skeleton information modelled to joint angular displacement maps (JADM). JADM is spatio temporal presentation of a 3D skeleton information and is taken from our past works. This proposed strategy 3DHAR-Net is equipped for dealing with enormous action sequences to predict the action classes by analysing series of sub-actions in time sequences. The entire experimentation is carried on our self-made 3D human activity datasets KLYoga3D and KLHA3D-102 action dataset and accomplished better outcomes and our method is further evaluated on the three benchmark datasets CMU, HDM05 and G3D.
\end{abstract}

Index Terms: Action recognition, Joint distance, Joint Angular displacement, Deep learning. 


\title{
A Quad Joint Relational Feature for 3D Skeletal Action Recognition with Circular CNNs
}

\author{
M. Teja Kiran Kumar' ${ }^{1}$ P.V.V. Kishore ${ }^{2}$ \\ ${ }^{12}$ Department of Electronics and Communications Engineering 1 Koneru Lakshmaiah \\ Educational Foundation (KLEF) Deemed to be University Vaddeswaram, Guntur, AP., India \\ Email: mtejakiran@kluniversity.in,pvvkishore@kluniversity.in
}

\begin{abstract}
:
To deal with the limitations of human action recognition systems that apply deep neural networks (DNNs) to 3D skeletal feature maps, we propose an improved set of features that enable better pattern discrimination when using a spectrally enriched circular convolutional neural network $(\mathrm{CCNN})$. These new features exploit the local relationships between joint movements based on 3D quadrilaterals constructed for all possible sets of four joints. Next, we compute the volumes of these time-varying quadrilaterals, by generating color-coded images, named spatio-temporal quadjoint relative volume feature maps (QjRVMs). To preserve the pixel frequency distribution while training a DNN, which is otherwise lost due to vanishing gradients and random dropouts, we propose a new architecture CCNNs. CCNNs use cyclic multi-resolution filters in a four-stream architecture, requiring only batch normalization and ReLU operations to identify multiple pixel pattern variations simultaneously. Applying the proposed CCNN to QjRVM images illustrates that combining multi-resolution features enhances the overall classification accuracy. Finally, we evaluate our proposed human action framework using our own 102-class, 5-subject action dataset, created using 3D motion capture technology, named KLHA3D-102. We also evaluate our framework using 3 publicly available datasets: CMU, HDM05, and NTU RGB-D.
\end{abstract}

Index Terms: Action recognition, Joint distance, Joint Angular displacement, Deep learning. 


\title{
A Real Time Prototype Model for the Security Features in the ATM
}

\author{
A.V. Prabu ${ }^{1}$, Kavati Srinivas ${ }^{2}$, Kandi Sambasivarao ${ }^{3}$ \\ ${ }^{1}$ Associate Professor, Department of ECE, Koneru Lakshmaiah Education Foundation, \\ Vaddeswaram, AP, India-22502. \\ ${ }^{2}$ Student M.tech, Department of ECM, Koneru Lakshmaiah Education Foundation, \\ Vaddeswaram, AP, India-22502. \\ ${ }^{3}$ Student M.tech, Department of ECM, Koneru Lakshmaiah Education Foundation, \\ Vaddeswaram, AP, India-22502. \\ Email id: ${ }^{1}$ prabu.deva@kluniversity.in
}

\begin{abstract}
:
At present days ATM has become prominent technology for banking sectors. It can do things like depositing of money, with drawling of money, fund transferring and other banking services. It is one of the crucial aspects of our financial activities. The growth rate of ATM units is exponentially high because of their multifunctional tasks. As we all know with the increase of ATM units the thefts occurred in the ATM units are also increased. So, it needs to be secure by technologically and physically. The Banking sector has already secured from the software related thefts. But ATM is the place where it is vulnerable to the physical attacks from the culprits. The proposed system provides security to the ATM as well as customers from the physical attacks and it is designed on the idea of to catch the thief inside the ATM centre only. The system is integrated with microcontroller unit, pressure sensor, and temperature sensor. Whenever any irrelevant things happened to the ATM unit from the burglars then immediately the sensors send signal to the microcontroller unit. Once the micro controller receives the signal from the sensors it do simultaneous things like closing the door, leakage of gas using solenoid valve, sending alert message to the higher authorities and police station by using GSM and GPS technology, activating the buzzer and led section for visual warnings. The closed door will not open until unless the correct password is given to the system with the help of keypad. One emergency button is fixed on the top of ATM to protect the user from the blackmailers.
\end{abstract}

Keywords: ATM security, pressure sensor, temperature sensor, GSM\&GPS, solenoid valve 


\title{
Energy Management System (EMS) Using Internet of Thing
}

\author{
A.V.Prabu' ${ }^{1}$, Y.L.Sarath Chandra ${ }^{2,}$ G.Sateesh Kumar ${ }^{3}$, Shaik Zeenath ${ }^{4}$ \\ ${ }^{1}$ Associate Professor, Department of ECE, Koneru Lakshmaiah Education Foundation, \\ Vaddeswaram, AP, India-22502. \\ ${ }^{2}$ Student, Department of ECE, Koneru Lakshmaiah Education Foundation, Vaddeswaram, \\ Guntur-522502, India. \\ ${ }^{3}$ Professor, Department of ECE, AITAM, Tekkali, Andhra Pradesh, India. \\ ${ }^{4}$ Student, Department of Electronics and Computer Engineering, Koneru Lakshmaiah Education \\ Foundation, Vaddeswaram, Guntur-522502, India. \\ Email id: prabu.deva@kluniversity.in
}

\begin{abstract}
:
In this work a residential energy management system is introduced and, that was developed through raspberry pi, wireless communication technology. An efficient method is proposed to monitor and control power consumption levels in the home. The home appliances are connected to $\mathrm{Wi}-\mathrm{Fi}$ hubs in the room, and these $\mathrm{Wi}-\mathrm{Fi}$ hubs communicate the power consumption levels and energy usage data. Based on the received data and users requirement, control commands are initiated, and the home appliances can be controlled automatically from the home server. Load sharing information will be displayed on the home server and webpage. If the power consumption levels exceed the user-determined threshold, the System will automatically switch off, and an SMS alert will be sent to the user.
\end{abstract}

Keywords: Residential Energy Management System, Raspberry pi, Wi-Fi, Home server, power consumption \& load sharing. 


\title{
Mathematical Modeling Approaches for Blockchain Technology
}

\author{
K. SureshKumar ${ }^{1}$, M. Geetha ${ }^{2}$, Ch.Vidyadhari ${ }^{3}$ \\ ${ }^{1}$ Department of ECE, ${ }^{2}$ Department of CSE, ${ }^{3}$ Department of IT \\ ${ }^{1}$ Koneru Lakshmaiah Education Foundation, Green Fields, Vaddeswaram, A.P., India- \\ 522502. ${ }^{2}$ College of Engineering and Technology, ${ }^{3}$ SRM Institute of Science and Technology, \\ Vadapalani, Chennai, Tamilnadu, India. \\ E-mail: m.k.sureshkumar@kluniversity.in.
}

\begin{abstract}
:
These days blockchain innovation has become a significant issue as of late and greatly affected the business. Despite the fact that blockchain innovations may bring us reliable and security issues, advantageous administrations, and difficulties behind this procedure assumes a significant key job that we have to break down. To have a superior observation about this square chain innovation, we will find out about the essential security highlights like-expanded limit, better security, unchanging nature, quicker settlement and decentralized framework. The range of blockchain applications limits from budgetary, human services, vehicle, chance administration, Internet of things to open and social administrations. In this paper, by examining its structure to various agreement calculations just as difficulties and openings, we endeavor to lead an expansive going review on blockchain innovation and also the few mathematical models behind this blockchain technology.
\end{abstract}

Index Terms - Blockchain, Security, Privacy, Bitcoin, Smart contract 


\title{
An Investigation on the Impact of Machine Learning in Wireless Sensor Networks and Its Application Specific Challenges
}

\author{
K. Praghash ${ }^{1}$,T. Karthikeyan ${ }^{2}$, K. SureshKumar ${ }^{1}$ \\ ${ }^{1,2}$ Department of ECE, ${ }^{1}$ Koneru Lakshmaiah Education Foundation, Green Fields, Vaddeswaram, \\ A.P,Annamacharya Institute of Technology and Sciences
}

\begin{abstract}
:
Abstract the importance of Machine Learning (ML) in advanced system technologies are proven in literature. This chapter investigates the role of ML in Wireless Sensor Networks and the challenges specific to its applications. We discuss the background literature of the renowned ML concepts and ML techniques. Further we distinguish the role of ML in WSN with detailed literature review. Subsequently, ML techniques for WSN are discussed from the literature. This chapter ends with the description of Functional and application specific challenges.
\end{abstract}

Index Terms -Machine Learning (ML) Application, Challenges, Wireless Sensor Networks (WSN) 


\title{
Model Assessment of Ionospheric Variability over Cyprus
}

\author{
Kanaka Durga Reddybattula ${ }^{1}$, Sampad Kumar Panda ${ }^{2}$ \\ ${ }^{1}$ Department of Atmospheric Sciences, Koneru Lakshmaiah Education Foundation, \\ Vaddeswaram, A.P., India-522502. \\ ${ }^{2}$ Department of ECE, Koneru Lakshmaiah Education Foundation, Vaddeswaram, A.P., India- \\ 522502. \\ E-mail ID: sampadpanda@kluniversity.in
}

\begin{abstract}
:
The present study focuses on ionospheric F-layer critical parameters (foF $/ \mathrm{hmF} 2$ ) and Total Electron Content (TEC) by exploiting a long-term data (2009 to 2016) from Digisonde and GPS receiver observations over Nicosia, Cyprus. It also includes performance evaluation of global empirical models and maps (IRI-2016; IRI-Plas 2017; GIMs). The results confirm a departure of estimated foF2/hmF2 and TEC in IRI-Plas, even larger than that of IRI-2016 as regards to the Digisonde and GPS observations, irrespective of season, solar activity, and geomagnetic activity conditions. This contradicts the general perception on an ideal behavior of the empirical climatological model over the middle latitude region. Also, we did not notice any convincing response of the storm sub-model in IRI except a rare flipping in its magnitude and absolutely no alteration in SPIM model to the onset of geomagnetic storms. This suggests a revisit into the underlying storm sub-model database in the models. Interestingly, the ingestion of Digisonde recorded foF2/hmF2 and GPS-TEC into the IRI-Plas model results in a remarkable convergence of the parameters towards the local observations even during active geomagnetic storm conditions. It ascertains the flexibility in IRI-Plas towards plausible improvements in the model estimations by ingesting GPS-TEC. The results are in line with efforts towards reliable ionospheric error mitigations in navigation, communication, and satellite applications.
\end{abstract}

Index Terms - IRI-2016, SPIM/IRI-Plas 2017, IGS-GIM, Digisonde, GPS. 


\title{
Ionospheric Total Electron Content variation over Saudi Arabian region during 6-10 September 2017 Solar flares and Associated Geomagnetic storms
}

\author{
Kanaka Durga Reddybattula ${ }^{1}$, Sampad Kumar Panda ${ }^{2}$ \\ ${ }^{1}$ Department of Atmospheric Sciences, Koneru Lakshmaiah Education Foundation, \\ Vaddeswaram, A.P., India-522502. \\ ${ }^{2}$ Department of ECE, Koneru Lakshmaiah Education Foundation, Vaddeswaram, A.P., India- \\ 522502. \\ E-mail ID: sampadpanda@kluniversity.in
}

\begin{abstract}
:
The present paper investigates ionospheric response to solar flares and geomagnetic storms during 6-10 September 2017 with total electron content (TEC) observations from six global positioning system (GPS) stations around Saudi Arabian low latitudes. The results show significant enhancement in TEC with the flare induced geomagnetic storms as compared to the average quiet days. An abrupt increase in TEC $(\sim 132 \%)$ is observed towards east (station-Oman) with a time lag of about $1 \mathrm{~h}$ from the brightest solar flare (X9.3) whereas other sites show enhancements within $22 \%-78 \%$, referring to a longitudinal asymmetry during the period. However, a remarkable deviation from the quiet time TEC is visible at all stations about $24 \mathrm{~h}$ after the event, with the maximum and the least positive differences in TEC at the westward Israel $(\sim 141 \%)$ and the southward Djibouti (23\%) stations, respectively. Comparable levels of TEC ( 200\%-300\%) were also seen at far equatorial locations after the M class flare on September 8, 2017, corroborating to the conjugate effects of solar flares and coronal mass ejections (CMEs) induced geomagnetic storms and $\mathrm{O} / \mathrm{N} 2$ changes. Interestingly, the second brightest solar flare (X8.2) appeared to be less effective confirming a negative ionospheric response towards east during the nighttime hours.
\end{abstract}

\section{Index Terms -Solar flare, Geomagnetic storm, Ionosphere Total electron content, Low latitude region}




\title{
Analysis of Non-Stationary Signals by Stockwell Transform
}

\author{
${ }^{1}$ B Murali Krishna \\ ${ }^{1}$ Department of ECE, Koneru Lakshmaiah Education Foundation, Vaddeswaram, A.P., India- \\ 522502. \\ E-mail ID: murali.ece713@kluniversity.in
}

\begin{abstract}
:
This paper presents the analysis of Cohen's class quadratic nonstationary signals by Stockwell transform. Stockwell transform (S-Transform) is an improved version for time frequency representation (TFR'S) of signals when compared with Short Time Fourier Transform (STFT), Continues Wavelet Transform (CWT), and Wigner Ville Distribution (WVD), etc. Hence STransform gains more popularity in now a days. In this paper a set of classical Cohen's type quadratic signals are considered and performed TFR'S with respect to S-Transform. Further these signals are tested under white guassian noise environment and evaluated the mean square error. The results show that $\mathrm{S}$-Transform gives better time frequency resolution at multiple frequency components level when compared with other TFR'S.
\end{abstract}

Keywords: Transform, FFT, Fourier, Time-Frequency. 


\title{
Time Frequency analysis of Non-Stationary signals by Differential frequency window S-Transform
}

\author{
${ }^{1}$ B Murali Krishna \\ ${ }^{1}$ Department of ECE, Koneru Lakshmaiah Education Foundation, Vaddeswaram, A.P., India- \\ 522502. \\ E-mail ID:murali.ece713@kluniversity.in
}

\begin{abstract}
:
The $\mathrm{S}$ transform is an extension of Short Time Fourier Transform and Wavelet transform, has a time frequency resolution which is far from ideal. A differential frequency window is proposed in this paper to enhance the time frequency energy localization. When a non-stationary signal consists of abrupt amplitude variation equal to peak of Gaussian function at initial intervals of chosen Gaussian window, then some part of the signal amplitude will be nullified during transform projection. The major function of differential frequency window is to track all abrupt amplitudefrequency variations which exploits in non-stationary signals. A mathematical method namely Newton Raphson method is adopted for this trace. The proposed scheme is tested for ECG data in presence of noise environment and results shows that proposed algorithm produces better enhanced energy localization in comparison to the standard S-Transform, STFT, and CWT. Furthermore the above algorithm is implemented on FPGA for real time applications.
\end{abstract}

Index Terms: STFT, Gaussian, Newton, FPGA. 


\title{
Analysis of an Energy-Balanced Underwater Acoustic Sensor Networks
}

\author{
V. Teju ${ }^{1}$, K.V.Sowmya ${ }^{2}$ \\ ${ }^{1,2}$ Department of ECE, Koneru Lakshmaiah Education Foundation, \\ Green Fields, Vaddeswaram, A.P., India-522502. \\ E-mail: ${ }^{1}$ teju@kluniversity.in, ${ }^{2}$ sowmyakambhampati@kluniversity.in
}

\begin{abstract}
:
Underwater Wireless Sensor Networks (UWSNs) have drawn tremendous concerns from all fields in mild in their huge application. Submerged remote sensor systems are like earthly wireless Sensor Networks (WSNs), be that as it is able to, due to numerous place of job and correspondence medium, UWSNs have several novel attributes, as an instance, excessive piece mistake price, lengthy begin to complete defer and coffee transfer pace. Those attributes of UWSNs purpose several troubles, for instance, retransmission, high power utilization and coffee dependability. To cope with those issues, many directing conventions for UWSNs are proposed. On this paper, an issue free steering convention, named power green directing convention depending on layers and inconsistent bunches (EERBLC) is proposed. EERBLC conference comprises of 3 ranges: layer and inconsistent agency association, transmission directing, maintenance and replace of bunches. Within the essential degree, the gazing zone under the water is partitioned into layers; the hubs in a comparable layer are bunched. For adjusting strength of the whole device and keeping far away from the "hotspot" difficulty, a unique inconsistent bunching approach depending on layers for UWSNs is proposed, wherein any other figuring technique for inconsistent institution length is exhibited. Inside the period in-between, every other bunch head dedication machine relying on power parity and degree is given. In the transmission degree, EERBLC convention proposes completely unique next forwarder strength of will technique depending on the sending percent and the lingering energy.
\end{abstract}

Index Terms: UWSN, EERBLC 


\title{
Enhancing Performance of IoT Networks through High Performance Computing
}

\author{
Dr. J, Sasi Bhanu1, Dr. JKR Sastry2 , P. Venkata Sunil Kumar3 , B. Venkata \\ Sai4 , K.V. Sowmya5 \\ ${ }^{1,2,3,4}$ Department of ECM, Koneru Lakshmaiah Education Foundation, \\ Green Fields, Vaddeswaram, A.P., India-522502. \\ ${ }^{5}$ Department of ECE, Koneru Lakshmaiah Education Foundation, \\ Green Fields, Vaddeswaram, A.P., India-522502. \\ E-mail: ${ }^{1}$ bhanukamesh1@gmail.com, ${ }^{2}$ drsastry@ kluniversity.in, \\ 5 sowmyakambhampati@kluniversity.in
}

\begin{abstract}
:
Performance of IOT based networks generally degrades as the system starts functions due to various reasons especially due to presence of heterogeneity; need to move the data from layer to layer in either of the directions to move the data from sensors to the storage of the same on remote server and to answer queries regarding the functioning of sensing and actuating mechanisms; the queries initiated from a remote location by the users through a Cloud computing interface. The performance degradation is generally due to the presence of heterogeneity and latency at each of the layers contained within an IOT network for forwarding the data packets either way. Maximum performance degradation is expected at gateway or restful services layer. The performance of an IOT network largely dependent of RESTful services layer especially the number of services to be supported are many in number. This paper presents a method of optimizing the performance of the IOT networks through implementation of High performance computing at RESTful services layer.
\end{abstract}

Key words: IOT networks, Performance optimization, Gateway, latency 


\title{
A Survey on Energy-Efficiency And Spectralefficiency in a Wireless Communication System
}

\author{
K. Nageswara Rao ${ }^{1}$ \\ ${ }^{1}$ Department of ECE, Koneru Lakshmaiah Education Foundation, \\ Green Fields, Vaddeswaram, A.P., India-522502. \\ E-mail: nagesh@kluniversity.in.
}

\begin{abstract}
:
In this paper we propose a basic approach to study the trade-off between energy efficiency (EE) and Spectral Efficiency (SE) in SIMO networks and several other tradeoffs with number of antennas, bandwidth, and channel gain. Both theoretical analysis and empirical results indicate that both the EE-SE trade-off efficiency significantly improved. Data traffic in a wireless communication is drastically increasing. The transmission of data is strongly related to energy usage in power amplifiers, processing of base band signal and due to transceiver hardware. The relation is measured by the Energy efficiency metric, expressed in bit/joule, which measures how much energy is consumed per information bit obtained correctly. Recent research papers usually prevent values of $100 \mathrm{Mbit} /$ joule, while past generations of network tend to function at energy efficiencies of 100Kbit/joule. Spectral efficiency usually is measured as bits/s/Hz.
\end{abstract}

Index Terms - Spectral efficiency, Energy efficiency, SIMO. 


\title{
BER analysis of communication system in the scattering scenario at mm-wave frequency range
}

\author{
K. Nageswara Rao ${ }^{1}$ \\ ${ }^{1}$ Department of ECE, Koneru Lakshmaiah Education Foundation, \\ Green Fields, Vaddeswaram, A.P., India-522502. \\ E-mail: nagesh@kluniversity.in.
}

\begin{abstract}
:
The millimeter waves(mm-wave) technology is playing key role in next-generation 5G wireless communication. The evolution of the mm-wave communication become crucial in scattering environment. In this paper, the performance of various multiple input multiple output (MIMO) simulated in terms of symbol error rate (BER). The more data rate high reliability, and reduction in the bit error rate with increase in the number of antennas both at transmitter and receiver. the SNR can be improved by array antenna and diversity gain. The SER performance in the presence of scatters and multiple antennas compared with the QPSK modulation
\end{abstract}

Index Terms - mm-wave, BER, QPSK, MIMO. 


\title{
Synthesis of unequally spaced linear antenna array using Modified Cat Swarm Optimization
}

\author{
Prasanna Kumar $\mathbf{K}^{1}$ \\ ${ }^{1}$ Department of ECE, Koneru Lakshmaiah Education Foundation, \\ Green Fields, Vaddeswaram, A.P., India-522502. \\ Email: prasannakumar@kluniversity.in
}

\begin{abstract}
In this paper, an updated cat swarm optimization (MCSO) is introduced that attributes quick convergence to efficient global search capabilities. In the modified location equation of cat swarm optimization (CSO), Gaussian mutation is added. The Gaussian mutation helps the CSO algorithm to check their positions to avoid premature convergence and problems with local optima. We have applied MCSO to regular, complex benchmark mathematical problems to demonstrate the efficacy of the proposed process. To decrease the degree of the maximum sidelobe and monitor null positions. Several examples of synthesis are considered and the results obtained are compared with linear aperiodic array designs. The numerical results obtained show that in terms of accuracy and convergence speed, along with reduced side lobe levels, the proposed method is superior to existing methods.
\end{abstract}

Key words : Communications systems, Peak sidelobe level, First Null Beam width, Modified Cat swarm optimization. 


\title{
Null Synthesis of Linear Antenna Array using Gaussian Mutated Cat Swarm Optimization
}

\author{
Prasanna Kumar $\mathbf{K}^{1}$ \\ ${ }^{1}$ Department of ECE, Koneru Lakshmaiah Education Foundation, \\ Green Fields, Vaddeswaram, A.P., India-522502. \\ Email: prasannakumar@kluniversity.in
}

\begin{abstract}
This paper presents a new technique for the null synthesis of an unequally spaced linear antenna array. To optimise the position of the antenna elements to suppress the degree of peak side lobe (PSLL) and to achieve nulls in desired directions, the Gaussian Mutated Cat Swarm Optimization (GMCSO) algorithm is implemented in this paper. A static penalty function is suggested that can put selective pressure on the PSLL, the first null beam width (FNBW) or the precise null positioning as needed by the application in hand, which gives a high degree of flexibility to the synthesis process. Numerical findings show that in terms of substantial reduction in the PSLL while retaining high nulls in desired directions, the proposed method outperforms different algorithms.
\end{abstract}

Keywords: Wireless systems, Gaussian Mutated Cat Swarm Optimization (GMCSO), Peak side lobe level (PSLL), First null beam width (FNBW). 


\title{
International Journal for Innovative
} Engineering and Management Research

A Peer Revieved Open Access International Journal

\section{Fuzzy Utilization in Speech Recognition and its Different Application}

\author{
J. Bennilo Fernandes ${ }^{1}$, KasiprasadMannepalli ${ }^{2}$ \\ ${ }^{12}$ Department of ECE, Koneru Lakshmaiah Education Foundation, \\ Green Fields, Vaddeswaram, A.P., India-522502. \\ Email: bennij05@gmail.com
}

\begin{abstract}
:
Talk affirmation is one among the basic zones in cutting edge talk process. The examination of talk affirmation may be a bit of an examination for "artificial intelligence" machines that may "hear" and "appreciate" the verbally communicated data. The customary ways for talk affirmation like HMM and DTW, are outrageously inconvenient and time excellent. As such formal Fuzzy justification may be an endeavor in cutting edge talk process for the convincing portrayal of talk affirmation in a couple of utilization. The approach masterminded in the midst of this paper streamlines the utilization of fuzzy in talk affirmation and make the data dealing with time shorter. The case considered in the midst of this paper is that the least mind boggling, i.e., the example of speaker dependence, little vocabulary, and disconnected words. There are various spectral and common choices isolated from human talk. The present ways for tendency acknowledgment from voice use basically MFCC and Energy feature. This paper briefs an overview concerning the present work on talk feeling ID strong for completing more examination by feathery approach.
\end{abstract}

Index Terms: Emotional Speech Recognition, Fuzzy, HMM, NN, Applications. 


\title{
Emotion Recognition Using Knn
}

\author{
BenniloFernandes.J ${ }^{1}$, Ch. Bhargavi ${ }^{2}$, S. Arshad ${ }^{3}$, G. Sai Kumar ${ }^{4}$, G. Sandeep ${ }^{5}$ \\ ${ }^{1}$ Department of ECE, Koneru Lakshmaiah Education Foundation, \\ Green Fields, Vaddeswaram, A.P., India-522502. \\ Email: bennij05@gmail.com
}

\begin{abstract}
:
The human speech will offer totally different styles of records regarding the speaker and speech. The emotional state recognition helps an individual to identify their health conditions and mental stress. In this paper different emotions like happy, angry, fear, sad \& normal is examined for recognition. With this approach, a new database is collected form 8 students in different combinations like male \& female and utilized for analysing. For any speech identification or recognition feature extraction and classification acts as the key factor, in this paper MFCC based Vector quantisation is used for extracting the features and KNN classification for recognition accuracy is utilised. From the feature extraction of MFCC, the simulation results shows $84.3 \%$ of overall accuracy \& in which emotional state like - Normall gives high recognition rate of $90 \%$.
\end{abstract}

Index Terms: Speech Recognition, MFCC, Vector Quantisation, KNN. 


\title{
Design of Magnitude Comparator Using Adiabatic Logic in ECRL
}

\author{
K. Priyanka ${ }^{1}$, P. Sathwik ${ }^{2}$, S. Sai Rahul ${ }^{3}$, Inumula Veeraraghava Rao ${ }^{4}$ \\ ${ }^{4}$ Department of ECE, Koneru Lakshmaiah Education Foundation, \\ Green Fields, Vaddeswaram, A.P., India-522502. \\ E-mail:raghva.inumula@kluniversity.in
}

\begin{abstract}
:
Efficiency of energy has become a major design to involve in high performance and mobile computer systems in this paper, a 2-bit comparator circuit is proposed by using with ECRL (Efficient Charge Recovery Logic). The term adiabatic is thermodynamics process that which recovery of energy and no exchange of energy occurs between system and environment. This design has improvement in power delay product from $65.76 \%$ to $83.02 \%$ at $1 \mathrm{~V}$ to $2 \mathrm{~V}$ when it is compared with the conventional design. The power dissipation will be reduced in adiabatic logic comparing with conventional CMOS logic. Recreation results uncover that the 2-bit ECRL (Efficient Charge Recovery Logic) comparator is $14.4 \%$ increasingly effective in power utilization when contrasted with the CMOS comparator, though a 2-bit PFAL comparator is $56.4 \%$ more effective in power utilization than the CMOS comparator. This result carried on Mentor Graphics. In adiabatic circuit the charge put away in load capacitor is recouped while in ordinary CMOS it is moved to ground which causes wastage of vitality. Adiabatic logic offers an approach to reuse the vitality put away in the load capacitors instead of the conventional method for releasing the capacitors to the ground and squandering this vitality. Results show a noteworthy improvement as far as PDP for proposed configuration as looked at to existing traditional plans.
\end{abstract}

Index Terms: ECRL, CMOS Logic, Adiabatic Logic, PDP 


\title{
DESIGN OF XOR and XNOR Gates Based on Full Adder
}

\section{Circuits}

\author{
V. Kavya Chowdary ${ }^{1}$, K. Sai Nishitha ${ }^{2}$, V. Naveen Sai ${ }^{3}$, Inumula \\ Veeraraghava Rao ${ }^{4}$ \\ ${ }^{4}$ Department of ECE, Koneru Lakshmaiah Education Foundation, \\ Green Fields, Vaddeswaram, A.P., India-522502. \\ E-mail:raghva.inumula@kluniversity.in
}

\begin{abstract}
This paper has a XOR / XNOR gate circuits produces separate and establishes a simultaneous XOR - XNOR function. Due to stub by yield capacity and shortcircuit energy dissipation, the power utilization and latency of these circuits is increasing A new one-bit adder hybrid circuit is chosen built on the effective gates of xor, xnor or xor / xnor. Each circuit is preferable because of its own advantages as it is known for its high speed, low current drain, short delay product (PDP), galvanic ability, etc. Simulations of the planned models were carried out using Mentor Graphics to see the quality of these projects. The simulation results are based on the 130-nm CMOS engineering design. A recent technique of transistor sizing is implemented to improve the circuits ' PDP.Today, predominant electronic systems are treated separately in every part of life and the usage of portable devices has been increased tremendously. These devices require to have less power utilization and high speed. While designing a system, power utilization is a parameter which is to be rectified for better system performance. The efficiency that depends on the execution of divider, comparator, multiplier, and adder which comes under arithmetic circuit which in turn shows the impact on numerous digital applications.
\end{abstract}

Index Terms: Transistor sizing method, PSO, XOR-XNOR, Output driving capability, DPL 


\title{
Evaluation of Adverse Effects of Fog on FSO links
}

\author{
A.Sree Madhuri ${ }^{1}$, Govardhani.Immadi ${ }^{2}$, M.Venkata Narayana ${ }^{3}$ \\ ${ }^{1,2,3}$ Department of ECE, Koneru Lakshmaiah Education Foundation, \\ Green Fields, Vaddeswaram, A.P., India-522502. \\ E-mail: asreemadhuri@kluniversity.in.
}

\begin{abstract}
:
The adverse effects of fog on FSO links is estimated with the help of simulation and experimental setup. The simulation and experimental results has followed a similar pattern and the pattern was like as the attenuation was increasing the signal strength started to deteriorate. This clearly indicates the need for a prior study of atmospheric effects on FSO link before being implemented in the real scenario. As FSO links were already in use for intersatellite communication purpose they will also be utilized for the earth station to satellite links in the near future which increases the necessity to study the link behavior.
\end{abstract}

Index Terms - Fog, link, communication, experimental setup, attenuation. 


\title{
Understanding the effects of various modulation techniques on FSO links
}

\author{
A.Sree Madhuri ${ }^{1}$, Govardhani.Immadi ${ }^{2}$, M.Venkata Narayana ${ }^{3}$ \\ ${ }^{1,2,3}$ Department of ECE, Koneru Lakshmaiah Education Foundation, \\ Green Fields, Vaddeswaram, A.P., India-522502. \\ E-mail: asreemadhuri@kluniversity.in.
}

\begin{abstract}
:
FSO utilizes the light propagating in the free space to transmit the data wirelessly for both computer networking and communication domains. In optical communication, we use optical fibre cable as a medium but in the free space optical communication, we use the free space as the medium to propagate the light signal. Here free space means air, or outer space. Free Space Optics is a line of sight communication. Here the link is in the range of 500 meters, 1500 meters and 2000 meters, a CW laser of $10 \mathrm{db}$ and at wavelength of $1550 \mathrm{~nm}$ is constructed under frail disturbance like climatic initiated attenuation ,scattering is assessed through different modulation techniques, to be specific ASK, PSK, FSK, Dual Drive MZ.
\end{abstract}

Index Terms - Sacttering Fog, link, communication, experimental setup, attenuation. 


\title{
Reduction of Peak to Average Power Ratio in OFDM System by Using Selected Mapping Technique
}

\author{
T Penchala Naidu ${ }^{1}$, M Venkata Narayana ${ }^{2}$ \\ ${ }^{1}$ Assistant Professor, Department of ECE, Koneru Lakshmaiah Education Foundation, \\ Vaddeswaram, A.P., India-522502. \\ ${ }^{2}$ Professor, Department of ECE, Koneru Lakshmaiah Education Foundation, Vaddeswaram, \\ A.P., India-522502. \\ Email: penchalanaidu@kluniversity.in
}

\begin{abstract}
:
The primary disadvantages of OFDM is high PAPR. The chose planning procedure is one of the most famous PAPR decrease plans for the OFDM framework. The current plan, which is utilized to decrease PAPR, is separating. In Filtering plan, there might be a chance of sign misfortune. So the separating strategy is certifiably not a best procedure to lessen PAPR in OFDM framework. Another procedure used to decrease PAPR is cutting. In the cut-out cycle, the first sign might be lost. The computational multifaceted nature of both separating and cutting is less, yet correspondence framework execution will endure with the utilization of these strategies because of the loss of wanted sign. Presently the proposed procedure is SLM. In the SLM procedure, the regulated data is duplicated with various stage arrangements lastly, we select the increased stage information with negligible PAPR. The OFDM framework with SLM is to some degree complex however the estimation of PAPR will be decreased. The other huge disadvantage of the OFDM framework is ISI. The ISI is there due to the multipath impact. To diminish the ISI content, we are utilizing the RAKE recipient. The beneficiary is planned so that it gets all the multipath signals, at that point does the time scaling to arrange all the multipath signals, lastly midpoints all the timescaled multipath signals. The proposed method is SLM. The computational unpredictability of translating OFDM with SLM is substantially less than that of OFDM framework. The recreation results show the PAPR decrease execution.
\end{abstract}

Index Terms: OFDM-Orthogonal Frequency Division Multiplexing, PAPR-Peak to Average Power Ratio, ISI-Inter Symbol Interference, SLM-Selected Mapping. 


\title{
Stochastic-Geometry Based Characterization of Aggregate Interference in TVWS Cognitive Radio Networks
}

\author{
Madhukar M. Deshmukh ${ }^{1}$, S. M. Zafaruddin ${ }^{2}$, AlbenaMihovska ${ }^{3}$,Ramjee \\ Prasad $^{4}$ \\ ${ }^{1}$ Department of ECE, Koneru Lakshmaiah Education Foundation, \\ Green Fields, Vaddeswaram, A.P., India-522502. \\ E-mail: madhukar_aau@kluniversity.in
}

\begin{abstract}
:
In this paper, we characterize the worst-case interference for a finite-area TV white space heterogeneous network using the tools of stochastic geometry. We derive closed-form expressions on the probability distribution function (PDF) and an average value of the aggregate interference for various values of path loss exponent under Rayleigh fading channel. The proposed characterization of the interference is simple and can be used in improving the spectrum access techniques. Using the derived PDF, we demonstrate the performance gain in the spectrum detection of an eigenvalue-based detector for cognitive radio networks. In this paper, we study to characterize the worst-case interference for a finite-area TV white space heterogeneous network using the tools of stochastic geometry under Rayleigh fading channel. The derived complimentary cumulative distribution function (CCDF) has a simple form, which can be used to obtain the outage performance of the network. We derive closed-form expressions on the PDF of the aggregate interference for various values of the path-loss exponent $\alpha=\{2,3,4,6\}$. The derived PDF is useful in improving the spectrum-sensing techniques, and power control for CR networks. We also derive the expected value of the aggregate interference for a receiver impacted by randomly distributed transmitters.
\end{abstract}

Index Terms: Rayleigh fading, path-loss, stochastic geometry 


\title{
Extreme Eigenvalue Based Detection Under Impact of Noise
} and Interference Uncertainty Madhukar M. Deshmukh ${ }^{1}$, AlbenaMihovska ${ }^{2}$, Ramjee Prasad ${ }^{3}$

${ }^{1}$ Department of ECE, Koneru Lakshmaiah Education Foundation, Green Fields, Vaddeswaram, A.P., India-522502.

E-mail: madhukar_aau@kluniversity.in

\begin{abstract}
:
The spectrum sensing in cognitive radio networks is challenging due to the requirement of signal detection under very low signal to noise ratio(SNR) and further due to the distributional uncertainty in the aggregate interference. In this paper our main objective is to design detection mechanism with performance, fairly independent of SINR, higher accuracy and medium algorithmic complexity. Secondly, the detector has ability to perform better in the presence of distributional uncertainty of non-Gaussian interference. The present work employs the cantering matrix to scale Sample Covariance matrix with Wishart distribution. In this work, the performance of the proposed detection algorithm is evaluated for signals under different modulation schemes and under different fading environment. Also, a realistic DVBT-2K signal has been used to evaluate the performance of proposed algorithm. Furthermore, distributional uncertainty in the non-Gaussian interference is quantified by calculating the differential entropy of the known PDF of interference and this is used to calculate the new threshold. In this work, we have used the cantering matrix to scale Sample Covariance matrix with Wishart distribution because an asymptotic analysis of the eigenvalues gives only the idea of presence or absence of extreme eigenvalues outside the limiting support space of the distribution. However, the probabilistic behaviour of the extreme eigenvalues and the spacings between eigenvalues are noteworthy in the case of signal detection and identification. It is observed that the maximum-minimum eigenvalue (MME) based proposed algorithm performs better than other proposed schemes in the literature on account of probability of detection and complexity. Effect of uncertainty quantification is evaluated in terms of ability to detect the signal in presence of non-Gaussian interference shows improved performance.
\end{abstract}

Keywords:SINR, Spectrum sensing, eigenvalues, non-Gaussian 


\title{
MACHINE LEARNING BASED SUBSURFACE ANALYSIS
}

\author{
V.G.K.Tilak ${ }^{1}$ and V.S.Ghali ${ }^{2}$ \\ ${ }^{12}$ Department of ECE, Koneru Lakshmaiah Education Foundation, \\ Green Fields, Vaddeswaram, A.P., India-522502. \\ E-mail: gvs0raos@kluniversity.in
}

\begin{abstract}
:
Qualitative defect detection with increased reliability in non-stationary stimulation based infrared non-destructive testing propels towards interesting post-processing research. In the recent past, machine learning has been evolved as a data processing approach in conventional and nonstationary thermal wave imaging modalities. On the other hand, thermal profiles contributing enough contrast are responsible for defect detection in processing techniques whereas, deeper and smaller defects present less contrast and are difficult to detect. From a machine learning point of view, defective profiles can be treated as outliers in contrast with the non-defective profiles. This paper introduces an unsupervised anomaly detection algorithm; local outlier factor (LOF) that computes the relation between thermal profiles as an outlier factor in contrast with its local neighbors, which is further used to classify defective and non-defective regions. Thermal response from a carbon fiber reinforced polymer sample with flat bottom holes of different sizes obtained by quadratic frequency-modulated optical excitation is used to validate the proposed methodology. Further, the signal to noise ratio and defect size estimation is used as a figure of merits to compare the defect detection performance of LOF with conventional defect detection algorithms.
\end{abstract}

Index Terms: Defect detection, non stationary, thermal wave imaging, local outlier factor. Carbon fiber reinforced polymer 


\title{
CHIRP-Z TRANSFORM BASED DEFECT DETECTION
}

\author{
V.S.Ghali ${ }^{1}$ and B.Suresh ${ }^{2}$ \\ ${ }^{1}$ Department of ECE, Koneru Lakshmaiah Education Foundation, \\ Green Fields, Vaddeswaram, A.P., India-522502. \\ E-mail: gvs0raos@kluniversity.in
}

\begin{abstract}
:
The present study performs an experimental investigation on quadratic frequency modulated thermal wave imaging (QFMTWI) modality to detect defects in a composite structure. Flat bottom holes are drilled in the test sample to simulate artificial delaminations of different sizes in varying depths as defects to validate the detection capability of the proposed technique. A low peak power optical heat flux modulated by a band of low frequencies is deposited on the front side of the sample and an infrared camera is used to analyze the respective thermal response. In the recent past, various signal processing techniques evolved as post-processing techniques in non-stationary thermal wave imaging techniques. The three favorable signal processing techniques in QFMTWI, namely pulse compression, Random projection transform, and recently introduced chirp z transform have been applied to the raw thermal response and respective contrast parameter is analyzed for the defect detection. The performance of each technique was evaluated concerning the enhanced defect detectability and signal to noise ratio (SNR). In addition to enhanced defect detection, these processing algorithms offered enhanced depth resolution that favors the observer to differentiate defects at various depths. Thus the depth resolution performance of each technique was evaluated concerning the defect detection, signal to noise ratio, and defect size.

Index Terms: Chirp z transform, Quadratic frequency modulated thermal wave imaging,Signal to noise ratio, Defect detection.
\end{abstract}




\title{
Gradient Pyramid based Enhanced Defect Detection for Quadratic Frequency Modulated Thermal wave Imaging
}

\author{
Badugu Suresh $^{1}$, Md Pasha $^{2}$, SkSubhani,G.V.P Chandra Sekhar ${ }^{3}$,V. S.Ghali ${ }^{4}$ \\ ${ }^{1}$ Department of ECE, Koneru Lakshmaiah Education Foundation, \\ Green Fields, Vaddeswaram, A.P., India-522502. \\ E-mail: sureshbadugu@kluniversity.in.
}

\begin{abstract}
:
Active thermography is an emerging nondestructive evaluation modality to inspect subsurface features of the stimulated test object using temporal temperature contrast over it. But captured thermal history may not explore all the subsurface anomalies due to non-uniform radiation, nonuniform emissivity and insuffient thermal contrast embedded in noise from deeper anomalies which demands the application of various signal and imaging processing techniques. But no single post processing method has been found to exhibit all the details with better contrast. Hence a modality to better explore all the details by embedding the details extract from variousprocessing methods is to be investigated. Data fusion facilitates us to embed the features obtained from various post processing modalities for anomaly detection and provides an enhanced defect detection capability in non-stationary thermal wave imaging. In order to obtain better subsurface details like location and size of defect, various post processed thermal features are merged using fusion techniques like wavelet and curvelet transform methods, Gradient Pyramid based fusion.This paper proposes a Gradient Pyramid based fusion methodology to combine the details obtain from various subsurface analysis approaches for quadratic frequency modulated thermal wave imaging through simulation carried over a CFRP sample and compares it with existing wavelet based fusion methodology using defect signal to noise ratio.
\end{abstract}

Index Terms - Active Thermography, Quadratic Frequency Modulated Thermal Wave Imaging, Feature Separation, Gradient Pyramid, FFT phase and pulse compression, Data fusion. 


\title{
Principle Component Analysis for Non Stationary Thermal Wave Imaging
}

\author{
Badugu Suresh $^{1}$, Md Pasha ${ }^{2}$, SkSubhani,G.V.P Chandra Sekhar ${ }^{3}$,V. S.Ghali ${ }^{4}$ \\ ${ }^{1}$ Department of ECE, Koneru Lakshmaiah Education Foundation, \\ Green Fields, Vaddeswaram, A.P., India-522502. \\ E-mail: sureshbadugu@kluniversity.in.
}

\begin{abstract}
:
Active thermography makes use of defect originated thermal contrast provided by stimulated test object surface for detection of anomalies. The weak and noise embedded thermal contrast obtained in this detection methodology demands various activation and processing approaches for extraction of fine subsurface details. Among variety of available stimulation approaches Quadratic Frequency Modulated Thermal Wave Imaging (QFMTWI) proved its depth resolution capability along with FFT phase and pulse compression processing approaches. These conventional approaches improves signal to noise ratio and enhances defect detection capability. Present contribution introduces a novel Principal component analysis based processing approaches for QFMTWI, which enhances detestability.
\end{abstract}

Index Terms -Active Thermography, Quadratic Frequency Modulated Thermal Wave Imaging, Feature Separation, Principal component analysis. FFT Phase and pulse compression. 


\title{
Impact of light soaking on absorber and buffer layer in thin film solar cells
}

\author{
Atul Kumar ${ }^{1}$, Pranay Ranjan ${ }^{2}$ \\ ${ }^{1}$ Department of ECE, Koneru Lakshmaiah Education Foundation, \\ Green Fields, Vaddeswaram, A.P., India-522502. \\ ${ }^{2}$ Department of Physics, UAE University, Abu Dhabi, 15551, United Arab Emirates \\ ${ }^{1}$ E-mail: atulkumar@kluniversity.in.
}

\begin{abstract}
:
Light soaking in thin film solar cells shows both beneficial and detrimental impact on efficiency depending upon the absorber-buffer pair. We here numerically simulated the advantageous and detrimental impact of light soaking behavior as experimentally observed in the literature by utilizing suitable defect models for the two cases, respectively. The proposed models are based on the photoconductivity and metastable photo-induced activity of absorber and buffer layers. It is observed that light soaking in absorber layer vis-a-vis photo-induced defect in absorber is beneficial for device performance. Photo-generated defect in absorber acts as hole doping which is turn enhance the built-in voltage. The photoconductivity of the buffer layer is detrimental to the device performance. The photo-generated defects in the buffer cause lowering of fill-factor, current, accelerate interfacial recombination and depreciate overall efficiency.
\end{abstract}

Index Terms -Defect model, thin film solar cell, light soaking, SCAPS. 


\title{
Impact of selenium composition variation in CZTS solar cells
}

\author{
Atul Kumar ${ }^{1}$ \\ ${ }^{1}$ Department of ECE, Koneru Lakshmaiah Education Foundation, \\ Green Fields, Vaddeswaram, A.P., India-522502. \\ ${ }^{1}$ E-mail: atulkumar@kluniversity.in.
}

\begin{abstract}
:
A comparative performance analysis of selenium composition variation in $\mathrm{Cu}_{2} \mathrm{ZnSn}\left(\mathrm{S}_{\mathrm{X}} \mathrm{Se}_{1-\mathrm{X}}\right)_{4}$ is performed in this paper. We obtained the optimal stoichiometry $\left(\mathrm{Cu}_{2} \mathrm{ZnSnS}_{1.8} \mathrm{Se}_{2.2}\right)$ where efficiency is highest. Comparative simulation brings out the trend of device characteristics variation with stoichiometry. The comparative simulation for band diagram shows the band banding at rear in CZTS whereas it is absent in CZTSe. This highlights the issue of back contact in pure sulphide CZTS. The cut off wavelength increases in QE plot as we move from CZTS to CZTSe compositions. The $\mathrm{V}_{\mathrm{OC}}$-illumination and $\mathrm{V}_{\mathrm{OC}}$-temperature plots shows (i) $\mathrm{V}_{\mathrm{OC}}$ saturation in CZTS and (ii) a linear rise of $V_{\text {OC }}$ for CZTSe. Nyquist impedance plot shows impedance decrease with selenium substitution. Se substitution enhances the chalcogenide opto-electronic properties as a whole and enhances devices performance. This enables higher efficiency of the CZTSSe devices as compared to the pure sulphide CZTS devices. An optimal stoichiometry obtained is $\left(\mathrm{Cu}_{2} \mathrm{ZnSnS}_{1.8} \mathrm{Se}_{2.2}\right)$ with efficiency of $15.3 \%$, pure selenide CZTSe has efficiency of $13.4 \%$ and $11.6 \%$ for pure sulphide CZTS solar cell.
\end{abstract}

Index terms: SCAPS, Band gap tune-ability, Chalcogenide, Solar cells. 


\title{
AAD TECHNIQUE to DETECT IONOSPHERIC ANOMALIES
}

\author{
M. Ravi Kumar ${ }^{1}$, M. Sridhar ${ }^{2}$, J. Lakshmi Prasanna ${ }^{3}$, K. Siva Krishna ${ }^{4}$, G. \\ Gnana Priya ${ }^{5}$ \\ 1,2,3,4,5 Department of ECE, Koneru Lakshmaiah Education Foundation, \\ Vaddeswaram, A.P., India-522502. \\ E-mail: ravikumar@kluniversity.in
}

\begin{abstract}
:
In this paper, Autonomous Anomaly Detection (AAD) is executed for recognizing ionospheric abnormalities from ground based Global positioning System (GPS) perceptions. EDA isbased on the troupe properties and shared conveyance of the observational discrete perceptions gives that characterization and inconsistency identification are autonomous,self -organizing,data driven and liberated from client and issue explicit parameters.Ionospheric code and transporter stage estimations recorded by Navigation with Indian Constellation (NAVIC) collector situated at Guntur, India is thought of. The code less transporter stage estimations are tried with proposed AAD strategy. It is discovered that AAD procedure is fit to catch ionospheric abnormalities in NAVIC L and S band signals. The result of this work would be helpful for improving positional precision of GPS clients.
\end{abstract}

Index Terms - NAVIC. 


\title{
ULTRASOUND IMAGES ENHANCEMENT BY SOBEL EDGE DETECTION
}

\author{
M Ravi Kumar', J Lakshmi Prasanna², Varanasi Sri Harsha Vardhan, \\ Morla Bhaskar Vystanava Swamulu ${ }^{4}$, Gade Kalyan Kumar ${ }^{5}$, Sreevardhan \\ Cheerla $^{6}$, Chella Santhosh ${ }^{7}$ \\ 1,2,3,4,5,6,7 Department of ECE, Koneru Lakshmaiah Education Foundation, \\ Vaddeswaram, A.P., India-522502. \\ E-mail: ravikumar@kluniversity.in
}

\begin{abstract}
:
Ultrasonic picture is an imaging method generally utilized in clinical demonstrative applications. Shockingly, the nature of ultrasonic pictures, primarily in view of spot commotion, is poor. We smother the sound of spikes utilizing picture channels in this undertaking lastly we make a picture of a ultrasound distinguished on an edge. Middle separating, Fourier channels, Sobel channels, histogram levelling, Canny Edge Detection are the methods utilized. Regarding edge security, the presentation of the proposed technique is determined by a mean basic closeness in connection to different strategies. The consequences of the proposed approach shows that the proposed approach can improve the picture nature of ultrasound pictures by improving picture quality while adequately limiting speckle noise.
\end{abstract}

Index Terms - Sobel channels. 


\title{
A MATLAB Approach for Design of Virtual Footwear
}

\author{
Namgiri Suresh ${ }^{1}$ \\ ${ }^{1}$ Department of ECE, Koneru Lakshmaiah Education Foundation, \\ Vaddeswaram, A.P., India-522502. \\ E-mail:namgirisuresh@kluniversity.in
}

\begin{abstract}
:
Image processing in the future will dramatically change the experience of the human brain. A vast number of applications, software, and techniques for image processing help extract complex image features. While image processing works beyond multidimensional tod ay and see what the image contains. Many techniques that draw on images in real time, but the real core is image processing. This paper addresses an overview of technologies, tools, and techniques for measurement of various foot images of human being and obtain the foot dimensions using image processing and EMD algorithm in MATLAB software. Measuring and valuing a distance between two points is important in the processing of objects. The idea is to make the Euclidean distance between two points a measure of how near (or distant) two points are to each other based on two ranges. Using this we can obtain the foot measurements with less efforts and in affordable price. The need to extract information from foot images and interpret their content was the driving factor in major footwear manufacturing industries and in health care for proving artificial legs.
\end{abstract}

Index Terms - EMD. 


\title{
Design of MEMS based Capacitive Pressure Sensorto Monitor the Respiratory Conditions of Patients
}

\author{
Namgiri Suresh ${ }^{1}$ \\ ${ }^{1}$ Department of ECE, Koneru Lakshmaiah Education Foundation, \\ Vaddeswaram, A.P., India-522502. \\ E-mail:namgirisuresh@kluniversity.in
}

\begin{abstract}
:
Healthcare plays a major role in enhancing aperson's quality of life. Continuous monitoring of healthcare is required especially in hospitals to keep an eye on one's health condition. Monitoring of a patient in hospitals and am bulancesis a vital task which involves lot of human intervention and cannot be reliable all the time. Hence, a need is felt to mechanize various monitoring activities. In this paper, we propose to institute a mechanism to monitor the various respiratory conditions of a given patient. This mechanism includes designing of a MEMS based capacitive pressure sensor which would help to monitor and diagnose various respiratory diseases based on the pressure of a person's exhaled breath. In this design, respiratory conditions are analysed based upon the displacement of the cantilever andcapacitance variation in the structural design.
\end{abstract}

Index Terms - MEMS. 


\title{
Impact of Ground Etching, Substrate and Inset Feed Height on Circularly Shaped Patch Antenna
}

\author{
Syed Inthiyaz ${ }^{1}$ \\ ${ }^{1}$ Department of ECE, Koneru Lakshmaiah Education Foundation, \\ Vaddeswaram, A.P., India-522502. \\ E-mail:syedinthiyaz@kluniversity.in
}

\begin{abstract}
:
Now a day the requirement of wireless communication increased rapidly with the demand for applications. This leads to the requirement of the design of compact wireless transceiver units. The antenna is one of the major blocks in the design which defines the compactness and efficiency ofthe unit. In this work, an elliptical patch antenna is designed etching the ground is studied. Also, the effect of inset feeding length studied. In the work, the author discussed various methods to convert a narrow band (NB) antenna into the ultra-wideband (UWB) antenna and wise versa. The proposed antenna can work in ultra band operation from $1 \mathrm{GHz}$ to $12 \mathrm{GHz}$. The same proposed structure can work a wide band of $5.8 \mathrm{GHz}$ bandwidth from $2 \mathrm{GHz}$ to $7.8 \mathrm{GHz}$ with inset feed.With the huge requirements of society, technology also developed rapidly. Especially in wireless communication systems, the growth is not having limits. With these unbounded limits, various wireless systems designs also increased. The major requirements are compacts, efficiency in terms of performance and power handling capabilities. The power handling capabilities can be improved by proper circuit design along with the antenna. But the performance of handling a wide band of allocation depends on the antenna design. The band of frequencies from $3.5 \mathrm{GHz}$ to $10.6 \mathrm{GHz}$ is approved as UWB by FCC in 2002. Various commercial applications come under this frequency band. So the design of the antenna in this band is a profitable challenge.
\end{abstract}

Index Terms - UWB, FCC, NB. 


\title{
Chexnet Reimplementation for Pneumonia recognition utilizing Pytorch
}

\author{
Syed Inthiyaz ${ }^{1}$ \\ ${ }^{1}$ Department of ECE, Koneru Lakshmaiah Education Foundation, \\ Vaddeswaram, A.P., India-522502. \\ E-mail:syedinthiyaz@kluniversity.in
}

\begin{abstract}
:
The chest $\mathrm{x}$-beam is one of the broadly performed indicative clinical imaging. A chest $\mathrm{x}$-beamis utilized to create pictures of the lungs, heart and the bones of the spine and chest. Chest x-beams utilize a limited quantity of ionizing radiation to create pictures of within the chest. It is mostly used to help analyze and screen treatment for various lung conditions, for example, pneumonia, emphysema, atelectasis, pneumothorax, cardiomegaly, pleural thickening, emanation, hernia, mass, edema, knob, union, fibrosis and invasion. One of the most perceived territories of programming improvement was to discover approaches to robotize clinical picture diagnostics by utilizing the current progressed uses of $\mathrm{AI}$ and profound learning. In this paper, we present a PyTorch based execution of the acclaimed CheXNet created by the Stanford University. The CheXNet model is a 121-Layer Convolutional Neural Network, otherwise called CNN. This CheXNet calculation is prepared on the greatest openly accessible dataset of numerous Chest Xbeam pictures (regularly known as CXR) called the ChestX-ray14 which comprises of more than 100,000 frontal view X-beams with named information of 14 illnesses gathered from more than 30,000 one of a kind patients. This dataset is gathered and kept up by National Institute of Health of United States of America. The CheXNet is known to analyze pneumonia at a rate that surpasses the degree of experienced human radiologists. The model is prepared to distinguish the 14 thoracic sicknesses.
\end{abstract}

Index Terms - CNN, CXR. 


\title{
International Journal for Innovative
} Engineering and Management Research

A Peer Revieved Open Access International Journal

\section{ANALYSIS OF OPTICAL LINK USING WAVELENGTH DIVISION MULTIPLEXING}

\author{
N.Durga Indira ${ }^{1}$ \\ ${ }^{1}$ Department of ECE, Koneru Lakshmaiah Education Foundation, \\ Vaddeswaram, A.P., India-522502. \\ E-mail:durgaindira@kluniversity.in
}

\begin{abstract}
:
The future in the field of communication the change will be faster. The wide range of variety technique implementing in WDM (wavelength Division Multiplexing) has taken. The reason behind to taken the optical fiber the attenuation, dispersion and total internal reflection. In the paper, we focused mainly on the intensity and its attenuations are calculated and accordingly the signal power and noise power link performance Q factor power and BER are calculated. Hence parameters will be calculated with BER Analyzer and the modulator is used. This examination is done in a simulation tool named OptiSystem and was improved by Optiwave.
\end{abstract}

Index Terms - Quality Factor, Signal Power, Noise power, Link performance 


\title{
Home-Appliances Monitoring based Wireless Gateway Network using Packet Tracer
}

\author{
N.Durga Indira ${ }^{1}$ \\ ${ }^{1}$ Department of ECE, Koneru Lakshmaiah Education Foundation, \\ Vaddeswaram, A.P., India-522502. \\ E-mail:durgaindira@kluniversity.in
}

\begin{abstract}
:
In the period of mechanical development nowadays, the innovation has become the requirement for the existence of the existing society. One is anticipated to make a keen domestic in turning on and rancid electronic devices by way of mobile cellphone. so far in killing and turning the home electronic device is completed via squeezing the transfer or far off trap, so answerable for electronic gadget manipulate less feasible. the home sensible shape is completed by means of reenactment idea by testing framework, prepare association, and far off domestic passage pc arrange tools required by way of a keen domestic gadget on cisco package tracer utilizing Internet Thing (IoT) control. In trying out the IoT home system faraway gadget entryway framework, diverse electronic devices may be controlled and checked via cell phone dependent on predefined arrangement conditions. With the clever home can conceivably construct vitality productiveness, decline energy usage costs, control hardware and trade the job of population.
\end{abstract}

\section{Index Terms - Wireless, Gateway, Packet, Switch, Server}




\title{
A Switchable Polarization Slot Antenna with DGS for WLAN/HIPERLAN Applications
}

\author{
D. Sreenivasa Rao ${ }^{1}$, Dr. I. Govardhani ${ }^{2}$ \\ ${ }^{1}$ Department of ECE, Koneru Lakshmaiah Education Foundation, \\ Vaddeswaram, A.P., India-522502. \\ E-mail: ${ }^{1}$ sreenuece@ kluniversity.in, ${ }^{2}$ govardhanee_ec@kluniversity.in
}

\begin{abstract}
:
This paper presents a $20 \times 35 \times 0.8 \mathrm{~mm} 3$ rectangular polarization reconfigurable antenna with four slots created at the corners on the patch. These four slots give the appropriate extra modes for circular polarization. Switching between LP and CP can be achieved using a PIN diode which is inserted across L-shaped slot in the ground. The designed antenna is optimized and analysed through simulations. The $10 \mathrm{~dB}$ impedance bandwidth and $3 \mathrm{~dB}$ axial ratio bandwidths are overlapped from 5.15 to $6.25 \mathrm{GHz}(19.3 \%)$. The protype antenna is tested in forward and reverse bias cases. It covers the bands IEEE 802.11a WLAN $(5.15-5.35 \mathrm{GHz}, 5.725-5.825 \mathrm{GHz})$ and HIPERLAN (5.45-5.725 GHz) and good compromise between simulated and measured results. The omni directional radiation diagrams of the antenna is suitable for wireless portable devices.
\end{abstract}

Index Terms - Reconfigurable Antenna, Polarization, Slots, PIN Diode 


\title{
A Controllable Band -Notched UWB Antenna with PIN Diodes for On -Demand Wi -MAX/WLAN/C/X Band Rejection
}

\author{
D. Sreenivasa Rao ${ }^{1}$, Dr. I. Govardhani ${ }^{2}$, M. Vasuja Devi ${ }^{3}$ \\ ${ }^{1}$ Department of ECE, Koneru Lakshmaiah Education Foundation, \\ Vaddeswaram, A.P., India-522502. \\ E-mail: ${ }^{1}$ sreenuece@kluniversity.in, ${ }^{2}$ govardhanee_ec@kluniversity.in, ${ }^{3}$ \\ vasujadevi@kluniversity.in
}

\begin{abstract}
:
In this work, a new controllable quadruple band -notched planar monopole antenna for ultra wideband applications is proposed. It is designed on 38 X $38 \mathrm{~mm} 2$ Fire -Resistant (FR - 4) epoxy substrate. It can avoid the inferences which rise s from the Wi -MAX $(3.3 \mathrm{GHz}-3.7 \mathrm{GHz}), \mathrm{C}$ band Satellite communication band $(3.9 \mathrm{GHz}-4.2 \mathrm{GHz})$, WLAN (5 GHz - $6 \mathrm{GHz}$ ), and X -band satellite communication uplink band $(7.9 \mathrm{GHz}-8.4 \mathrm{GHz})$. Four slots are inserted in the radiating element to achieve the band rejection characteristics. The effect $s$ of slot length and width on the band rejection characteristics are also studied. Slots are loaded with four RF microwave switches to control the band -notched characteristics. The designed antenna is optimized using electromagnetic simulation software Ansoft HFSS. The prototype antenna is fabricated and tested . The designed antenna operates in a band from 3.1 to over $11 \mathrm{GHz}$ and observed the good radiation characteristics. The simulated and measured results indicate that the proposed antenna is suited for portable devices.
\end{abstract}

Index Terms - Band notched, Reconfigurable, Slots, Ultra -Wide Band 


\title{
A Novel on Smart Antennas to improve performance in Wireless Communications
}

\author{
P. Saleem Akram ${ }^{1}$, Pritika Bhatt ${ }^{2}$, Dr. T.V. Ramana ${ }^{3}$ \\ ${ }^{1}$ Department of ECE, Koneru Lakshmaiah Education Foundation, \\ Green Fields, Vaddeswaram, A.P., India-522502. \\ Dept of ECE, GITAM University, Visakhapatnam, A.P, India. \\ E-mail: ${ }^{1}$ saleemakramp@kluniversity.in
}

\begin{abstract}
:
In wireless communication, a broadcast server serves a number of clients. The exchange of information from server and access by the clients takes place in a cyclic path using the directional antennas. But directional antennas have several drawbacks. So the multiple directional antennas were used to replace the fixed directional antenna systems. When using the multiple antennas we need to consider the geographical distribution of the clients over coverage area of system to get the desired improved performance. All these disadvantages motivate us to develop a technique named as a wireless push system developed using adaptive smart antenna with rescheduling application to be used at broadcasting server .Smart antennas can vary the beam widths according to client position using suitable algorithms. Beam width of the antenna is changed as per the changing position of the client and appropriate changes are introduced in the broadcasting schedules. This approach will bring the desired improvement in system performance.
\end{abstract}

Index Terms - broadcasting server, data broadcasting, demand probability, smart antennas 


\title{
Study of Changes in Antenna Performance when Subjected to Di-electric filled Metamaterials over the Frequency Range upto $50 \mathrm{GHz}$
}

\author{
P. Saleem Akram ${ }^{1}$, Dr. T.V. Ramana ${ }^{2}$ \\ ${ }^{1}$ Department of ECE, Koneru Lakshmaiah Education Foundation, \\ Green Fields, Vaddeswaram, A.P., India-522502. \\ Dept of ECE, GITAM University, Visakhapatnam, A.P, India \\ .E-mail ID: saleemakramp@kluniversity.in
}

\begin{abstract}
:
In this paper, we design a Metasurface to replace ground plane of the rectangular patch antenna. The Metasurface structure is capable of guiding energy in unpredictable way and it became very useful factor in working with EM energy and light energy. When subjected to changes in its designing parameters it exhibits significance change in the way it is manipulating the waves and the materials used also causes changes in its way of working and the di-electric filled or all dielectric thin Metasurfaces are considered in this work a coaxial fed rectangular patch antenna which operate at $10 \mathrm{GHz}$ is considered for analysis. And metasurface having $0.2 \mathrm{~cm}$ pin height and $0.2 \mathrm{~cm}$ gap between plates will fill with different dielectric substrates with dielectric constants 2.2 , 2.94 and 6.15 and comparative performance analysis is illustrated.
\end{abstract}

Index Terms - Metamaterials, patch antenna, di-electric substrate, frequency band, wave reflection and transmission 


\title{
Second Function Logic Circuits
}

\author{
J. Lakshmi Prasanna ${ }^{1}$, K.Naga Jaya Lasya ${ }^{2}$, M.Sohail Abbas, S.Sushmanth ${ }^{3}$ \\ ${ }^{1}$ Department of ECE, Koneru Lakshmaiah Education Foundation, \\ Green Fields, Vaddeswaram, A.P., India-522502. \\ E-mail ID: lakshmiprasannanewmail@kluniversity.in
}

\begin{abstract}
:
In this paper, the way to deal with plan of multifunctional computerized circuits is exhibited .It depends on reception of polymorphic hardware worldview which licenses advanced circuits to display in excess of one capacity while saving a similar structure .All things considered just segments of the circuit (gates) must be multifunctional. Singular gates have normally builtin effect to the event of a few wonders summoning the capacity change (e.g. control supply level and so on.), which implies that no devoted net is required for that reason .Besides,a first total arrangement of effectively re-enacted two input polymorphic inputs were considered. These logic gates demonstrate the best parameters of all the recently distributed polymorphic entryways high information impedance and low yield impedance, brief time flag spread, low power utilization and low transistor count being utilized. Extensive variety of proposed polymorphic gates (work mixes) may acquire more proficient outcomes amid synthesis. The proposed strategy we have utilized as $180 \mathrm{~nm}$ technology utilizing TANNER Tools.
\end{abstract}

Index Terms - TANNER 


\title{
Design of Logic Circuits using QCA
}

\author{
J. Lakshmi Prasanna ${ }^{1}$, R.S. Ernest Ravindran ${ }^{2}$, M. Ravi Kumar ${ }^{3}$, K. Sree \\ Pooja $^{4}$, U V S Sumanth ${ }^{5}$, Shaik Ahamed ${ }^{6}$ and Chella Santhosh ${ }^{7}$ \\ ${ }^{1}$ Department of ECE, Koneru Lakshmaiah Education Foundation, \\ Green Fields, Vaddeswaram, A.P., India-522502. \\ E-mail ID: lakshmiprasannanewmail@kluniversity.in
}

\begin{abstract}
:
QCA may be a technology that is semiconductor less and it's one amongst its kind and rising technology. operating of QCA strictly works on the principle of coulombic repulsions. thanks to these coulombic repulsions of electrons in quantum dots, high speed digital circuits is designed. when put next to existing CMOS technology, machine speed of Quantum Cellular Automata is considerably high. This paper primarily depicts the functioning and simulation results of BCD Adder which was designed victimization Quantum Cellular Automata. Digital Circuits with high complexness can be designed by using Quantum Cellular Automata by using crossovers. When crossovers and multi-layer connections are used space occupied by the circuit and delay time are reduced.
\end{abstract}

Index Terms - QCA 


\title{
Improvement of Image Reconstruction Using Multiscale Am- Fm Methods
}

\author{
K. T. P. S. Kumar ${ }^{1}$, Lakshman Pappula ${ }^{2}$, V. Sahithi ${ }^{3}$ \\ ${ }^{1}$ Department of ECE, Koneru Lakshmaiah Education Foundation, \\ Green Fields, Vaddeswaram, A.P., India-522502. \\ E-mail ID: satishkumar@kluniversity.in
}

\begin{abstract}
:
This paper discuss about new AM-FM methods for image reconstruction. This approach is based on 2 basic ideas: i) AM-FM Demodulation using new gaborfilterbank ii) New accurate methods for instantaneous frequency (IF) estimation. This project includes quasi-eigen function approximation(QEA), quasi local method(QLM) and variable-spacing local linear phase (VS-LLP) methods for improved accuracy. The new VS-LLP method is a generalization of QEA method where we choose the best integer spacing between the samples to adapt as a function of frequency. We also introduce QLM method for IF and IA estimation. We present different noises (salt\&pepper, speckle, poisson, Gaussian) decompositions to show that the proposed methods can be used to reconstruct and analyze general images. However each technique has different properties, making it more suited for specific applications and this technique is implemented by using MATLAB.
\end{abstract}

Index Terms - Amplitude modulation Frequency modulation(AM-FM), Image reconstruction, Instantaneuosfrequency,Multiscale 


\title{
Performance analysis of Absolute Deviation Filter for Removal of Impulse Noise
}

\author{
K. T. P. S. Kumar ${ }^{1}$, Lakshman Pappula ${ }^{2}$, V. Sahithi ${ }^{3}$ \\ ${ }^{1}$ Department of ECE, Koneru Lakshmaiah Education Foundation, \\ Green Fields, Vaddeswaram, A.P., India-522502. \\ E-mail ID: satishkumar@kluniversity.in
}

\begin{abstract}
:
This paper presents the process of removal of impulse noises using different median filters. This contains a vector median filter that includes a new mechanism for the detection of impulses in colour images prior to further processing operations. When compared to other filters, the proposed filter will be tested for images corrupted by two sided fixed impulse noise model, nothing but salt and pepper noise. If the central vector pixel in a kernel is found to be corrupted, it is replaced by the vector median of the kernel, else it is kept unchanged. The vector pixels in a specified window are ranked on the basis of sum of the distances between each vector pixel and the other vector pixels in the window. Since the detection mechanism uses absolute deviation values, the computational complexity is reduced. The results show a better performance of the proposed algorithm in lesser execution time as compared to a number of existing schemes for restoring colour images corrupted by impulse noise.
\end{abstract}

Index Terms - kernel; vector median filter; rank-conditioned vector median filter; rankconditioned and threshold vector median filter; centre-weighted vector median filter; two-sided fixed impulses noise model 


\title{
Large antenna array synthesis using modified differential evolution algorithm
}

\author{
Lakshman Pappula $^{1}$, K. T. P. S. Kumar ${ }^{2}$, V. Sahithi ${ }^{3}$ \\ ${ }^{1}$ Department of ECE, Koneru Lakshmaiah Education Foundation, \\ Green Fields, Vaddeswaram, A.P., India-522502. \\ ${ }^{1}$ E-mail ID: lakshman.pappula@ @luniversity.in
}

\begin{abstract}
:
The present work highlights the synthesis of large antenna arrays using the modified differential evolution algorithm. With the help of the modified differential evolution algorithm the phase and/or amplitude excitation of the individual elements present in an array are optimized. This optimization in performed to achieve low sidelobe levels (SLL) also to place the NULLS in the desired directions and the results are compared to that of the uniform linear array. After the analysis on results it is concluded that the above algorithm can be effectively utilized in the synthesis of large antenna arrays having more than 100 elements.
\end{abstract}

Index Terms - Large arrays, modified differential evolution, Sidelobe levels, Amplitude, Phase 


\title{
Design of Triangular Patch Antenna with reduced RCS for Stealth Applications using Bionics
}

\author{
Lakshman Pappula $^{1}$, V. Sahithi ${ }^{2}$, K.T.P.S Kumar ${ }^{3}$ \\ ${ }^{1}$ Department of ECE, Koneru Lakshmaiah Education Foundation, \\ Green Fields, Vaddeswaram, A.P., India-522502. \\ ${ }^{1}$ E-mail ID: lakshman.pappula@kluniversity.in
}

\begin{abstract}
:
In this paper Bionics principle is applied to the proposed triangular antenna to show the antenna radar cross section (RCS) reduction by the use of a model insect tentacle antenna. Bionics is the study of mechanical systems that function like living organisms or parts of living organisms. To authenticate this method, a novel triangular micro strip patch antenna with reduced RCS is proposed and simulated for stealth applications. Mono static RCS and Bi-static RCS of an insect tentacle antenna (ITA) of triangular shape is proposed and simulated. In this paper we have taken the Flame retarded substrate (FR4) which is made up of epoxy resin component, which is a very smooth substrate and is very cheaper to manufacture and gives better return loss. The substrate FR4 Epoxy used in this have a dielectric constant of 4.4. The results shown that the performance of the proposed triangular antenna has lower RCS and favourable radiation performances.

Index Terms - Micro strip patch antenna, Mono static RCS, Bi-static RCS, Return loss, Bionics, Stealth Technology
\end{abstract}




\title{
Location Monitoring System with Image Database
}

\author{
B.Srikanth Deepak ${ }^{1}$ \\ ${ }^{1}$ Department of ECE, Koneru Lakshmaiah Education Foundation, \\ Green Fields, Vaddeswaram, A.P., India-522502. \\ ${ }^{1}$ E-mail ID: deepak.bsd@kluniversity.in
}

\begin{abstract}
:
This paper presents a sharp distantly reasonable structure that is utilized for live assessment passageway watching utilizing Raspberry Pi and WhatsApp. [1] This is an Internet of Things (IoT) based model masterminded by utilizing Raspberry Pi. [1] In this proposed structure the reasonableness of executing certain basic tasks with higher trouble and far off access have been interwoven. Beginning late it is phenomenally hard to screen the specific region it requires a gigantic measure of human exertion and limit. In this paper, we proposed a discernment structure that dependably screens a specific region. The class Room Monitoring and controlling discernment camera through any Internet Services through twilio on the web minimized structure with the WhatsApp highlight. In this we propose a picture catching framework to catch required picture and store it Inbuilt Memory Card for highlight Reference. We can screen this from any place on the planet by using a wireless and WhatsApp presented in it. We join another Most Required Application for instance Home Automation through WhatsApp.
\end{abstract}

\section{Index Terms - Twilio, WhatsApp}




\title{
Control of Home Appliances using Mobile phone
}

\author{
B.Srikanth Deepak ${ }^{1}$ \\ ${ }^{1}$ Department of ECE, Koneru Lakshmaiah Education Foundation, \\ Green Fields, Vaddeswaram, A.P., India-522502. \\ ${ }^{1}$ E-mail ID: deepak.bsd@kluniversity.in
}

\begin{abstract}
:
This paper presents a quick distantly reasonable framework that controls the home apparatuses by utilizing WhatsApp. This is an IOT based model planned and created by utilizing Raspberry Pi-3. In this proposed arrangement of home computerization, the achievability of executing certain significant errands with higher controllability what's more, far off access have been fused. Home apparatuses such as lights, fans, forced air systems, power driven electrical and electronic wares and so forth, are made to control from any piece of this world, utilizing a cell phone with WhatsApp introduced in it. For guaranteeing this usefulness, a web interface has been created utilizing the Raspberry Pi, as a web worker for working furthermore, controlling the home machines through any Wi-Fi open cell phone with WhatsApp include.
\end{abstract}

Index Terms - Wi-Fi, Raspberry Pi-3 


\title{
Replica Redundancy Block Multiplier using Fixed Width
}

\author{
K.V.K.V.L.Pavan Kumar ${ }^{1}$, V.S.V. Prabhakar ${ }^{2}$, S. Vamsee Krishna ${ }^{3}$ \\ ${ }^{123}$ Department of ECE, Koneru Lakshmaiah Education Foundation, \\ Green Fields, Vaddeswaram, A.P., India-522502. \\ ${ }^{1}$ E-mail ID: pavan.cec@kluniversity.in
}

\begin{abstract}
:
In this paper, we propose a fixed-width multiplier plan by utilizing flexible commotion lenient ANT design that assists with building fixed-width multiplier with decreased exactness reproduction repetition block (RPR). The proposed design can accomplish high exactness, low force utilization, and region effectiveness. We give fixed-width RPR with blunder remuneration circuit utilizing the halfway item terms of information rectification vector to bring down the prune mistakes, the equipment for blunder remuneration circuit is basic. In a $12 \times 12$-digit multiplier, region of fixed-width RPR can be brought down by $44.55 \%$ and power utilization is spared by $23 \%$ as analyzed with the existed ANT plan.
\end{abstract}

Index Terms - RPR, ANT 


\title{
Dynamic Comparators an Overview
}

\author{
K.V.K.V.L.Pavan Kumar ${ }^{1}$, V.S.V. Prabhakar ${ }^{2}$, S. Vamsee Krishna ${ }^{3}$ \\ ${ }^{123}$ Department of ECE, Koneru Lakshmaiah Education Foundation, \\ Green Fields, Vaddeswaram, A.P., India-522502. \\ ${ }^{1}$ E-mail ID: pavan.cec@kluniversity.in
}

\begin{abstract}
:
This paper encases the presentation examination of dynamic predisposition based twofold tail lock and Elzakker's comparators utilizing HEP 1 by MENTOR GRAPHICS in 130nm CMOS measure. Force, delay and PDP of these comparators are contrasted and without utilizing dynamic inclination that outcomes in critical improvement of these boundaries Performance correlation of these boundaries use $500 \mathrm{MHz}$ recurrence. Dynamic predisposition comparators may scale back the normal energy utilization when contrasted with the earlier comparators. The twofold tail hook structure presented in extinguished these issues by separating the preamplifier from the hooking activity, though at the consistent time low voltage headroom interest and permits the activity at lower flexibly voltages.
\end{abstract}

\section{Index Terms - HEP, PDP}




\title{
CZT Precoding Based Hybrid Techniques to Reduce PAPR in WIMAX( IEEE 802.16)
}

\author{
Madhav Prasad Khanal ${ }^{1}$ \\ ${ }^{1}$ Department of ECE, Koneru Lakshmaiah Education Foundation, \\ Green Fields, Vaddeswaram, A.P., India-522502. \\ ${ }^{1}$ E-mail ID: khanalmadhav@kluniversity.in
}

\begin{abstract}
:
The popularity and flexibility of wireless communication makes WiMAX a revolutionary technique of next generation packet service. WiMAX adapts orthogonal frequency division multiplexing (OFDM) technique for multiple transmissions. WiMAX faces the problem of high peak to average power ratio (PAPR) like other OFDM based techniques. Linear precoding techniques and nonlinear techniques like signal scrambling techniques and signal distortion techniques are major techniques to reduce high PAPR. Linear precoding technique has advantage of no degradation in performance of system and can be used with nonlinear techniques. Hybrid techniques (Linear block precoding technique with nonlinear techniques) are proposed to be the more efficient technique to reduce high PAPR. Simulation results show reduced PAPR in proposed techniques with respect to other linear and nonlineartechniques.CZT precoding technique with nonlinear techniques (clipping, SLM and PTS) are used to analyze Peak to Average Power Ratio (PAPR) for different modulation schemes.
\end{abstract}

Index Terms -WiMAX, PAPR, CZT, SLM, PTS 


\title{
An Image Encryption and Decryption using Chaos Algorithm
}

\author{
Madhav Prasad Khanal' ${ }^{1}$ K. T. P. S. Kumar ${ }^{2}$ \\ ${ }^{1}$ Department of ECE, Koneru Lakshmaiah Education Foundation, \\ Green Fields, Vaddeswaram, A.P., India-522502. \\ ${ }^{1}$ E-mail ID: khanalmadhav@kluniversity.in
}

\begin{abstract}
:
In a well-developed digital world, security plays a key role in transmission of images. To overcome these challenges Encryption and Decryption techniques need to be applied. In this paper we have applied the chaotic encryption and chaotic decryption on an image by doing pixel shuffling and using chaotic maps. Chaotic nature i.e., randomness property is present in both Henon map and Arnold cat map. Pseudorandom values generation plays an important key role in Henon maps and iteratively pixel shuffling is done in Arnold cat map. A sorting Technique is followed on key values produced by Henon map. By using those sorted positions, shuffle the pixel values generated by Arnold cat map iteratively. In this way the images are provided with high security for confidential transmission.
\end{abstract}

Index Terms -Chaotic map, Pseudo-Random values, Pixel shuffling, sorting 


\title{
Review on Advancement of TDD LTE communication network and beyond
}

\author{
N.Prabakaran ${ }^{1}$ \\ ${ }^{1}$ Department of ECE, Koneru Lakshmaiah Education Foundation, \\ Green Fields, Vaddeswaram, A.P., India-522502. \\ ${ }^{1}$ E-mail ID: prabakaran@kluniversity.in
}

\begin{abstract}
:
The TDD-LTE is becoming one of the fast and best communication technologies to support 4G. The low cost in deployment of TDD-LTE made it preferable by network operators and hence its rapid growth in deployment. By 2013 more than153 TDD LTE networks have been commercially launched. In this paper we have studied the existing literature to map the evolution and technological advancement ofTDD-LTE. Existing literature has been reviewed to understand the developments in-features like Carrier Aggregation (CA), Further enhanced Inter-Cell Interference Coordination (FeICIC), Coordinated Multi-Point (CoMP), Enhanced MIMO, eIMTA, Device to Device(D2D) and minimisation of drive tests (MDT). TDD-LTE probable technological evolution towards $5 \mathrm{G}$ has also been reviewed and presented in this paper.
\end{abstract}

Index Terms - TDD-LTE, MIMO, CoMP, 4G and Review. 

Engineering and Management Research

A Peer Revieved Open Access International Journal

\title{
IMPLEMENTATION OF IOT BASED SMART SURVEILLIANCE MONITORING AND CONTROLLING SYSTEM
}

\author{
N.Prabakaran ${ }^{1}$ \\ ${ }^{1}$ Department of ECE, Koneru Lakshmaiah Education Foundation, \\ Green Fields, Vaddeswaram, A.P., India-522502. \\ ${ }^{1}$ E-mail ID: prabakaran@kluniversity.in
}

\begin{abstract}
:
This paper deals with the implementation of Smart surveillance monitoring method using Raspberry-Pi and PIR sensor. It can be used at homes, industries etc, where the audio and movement of objects are recorded by a Camera and transmit the data to the Server using Internet of Things. Raspberry Pi has two main mechanisms interacting with each other; one is the Web Application that executes on the mobile device browser and server-side scripts that run in a cloud which will be operated by the Raspberry Pi Hardware module. Hardware implementation has been done for the proposed model and it will be applicable for monitoring home, industry, office etc. in the absence of user.
\end{abstract}

Index Terms - Internet of Things, PIR sensor, Smart phone, Web Camera 


\title{
QCA Design and performance analysis of Binary to Excess code converter
}

\author{
B.John Philip ${ }^{1}$ \\ ${ }^{1}$ Department of ECE, Koneru Lakshmaiah Education Foundation, \\ Green Fields, Vaddeswaram, A.P., India-522502. \\ ${ }^{1}$ E-mail ID: johnphilip@kluniversity.in
}

\begin{abstract}
:
An efficient QCA Excess-3 code converter has been proposed for QCA. The proposed circuits can be efficiently used for data communication systems for reliable information communication at nanoscale. In CMOS devices, many complications arise such as quantum effects, short channel effects and power dissipation, when scaling down to submicron level. To overcome the limitations of CMOS devices, Quantum-cellular automata(QCA) technology is implemented to design the code converters. A well-optimized conversion technique like N-bit (typically 4-bit, 8-bit, 16-bit, 32-bit and 64-bit) Binary-to-Gray and a 4-bit Excess-3 code converter is explored for implementation using QCA. Attempt has been made to enhance the performance of conventional 4-bit Binary-to-Gray code converter. The proposed 4-bit Binary-to-Gray code converter has been compared with conventional designs and achieves better circuit performance in terms of area, circuit complexity and clock delays as depicted. A novel N-bit Binary-to-Gray code converter (for $\mathrm{N}=8,16,32$ and 64 ) has also been proposed in this paper. The proposed design enjoys the features of lesser area and reduced circuit complexity. Moreover, the proposed N-bit Binary-to-Gray code converter consumes very less clock delays as depicted in this paper. Efficient methods of interconnections for pipeline architecture structures have been considered. The design and simulation results of present work have verified using QCA Designer tool. The work conducted in this paper thus opens a wider path for digital circuit design with smaller circuit dimensions.
\end{abstract}

Index Terms - QCA, CMOS 


\title{
Validation of Rain Attenuation Time Series Synthesizers to Predict the Artificial Rain Attenuation for Propagation Impairments in Satellite Communications
}

\author{
B.John Philip ${ }^{1}$ \\ ${ }^{1}$ Department of ECE, Koneru Lakshmaiah Education Foundation, \\ Green Fields, Vaddeswaram, A.P., India-522502. \\ ${ }^{1}$ E-mail ID: johnphilip@kluniversity.in
}

\begin{abstract}
:
Whenever the signal attenuation due to rain is discussed, various forms of proposals have been brought forward. There are several models of attenuation that can be seen in the literature in the past, many of them are updated and there are still others to go; several of them are ITU rain model, DAH, Lognormal, Assis, Flavin, Crane, SAM, Moupfouma, Yamada, Lin, and so forth. The communication field of ITU radio (ITU-R) is one of the three branches of the International Telecommunications Union (ITU), which is responsible for radio transmission and regulates regional radio spectrum and satellite orbit resources, as well as improving radio communication network quality with the goal of ensuring the efficient usage of spectrum. Depending on data obtained from the United States, the ITU-R offers an estimation of the total distribution of rainfall in the planet by splitting it into five regions. To find out the rain attenuation at any particular region, some predefined calculations are necessary. i.e. from which height rain is falling, the nature of rainfall, etc. All these calculations are provided by the International Telecommunication Union. Hence, we prefer to use the ITU-R model. To develop and test real time Fade Mitigation Techniques control algorithms, propagation time series are needed. An alternative to using real data collected from propagation experiments is to generate typical fading time series making use of climatological characteristics as well as geometrical and radio-electrical parameters of the link. The aim of the study presented in this paper is to validate time-series synthesizers relying on an enhanced ITU-R model.
\end{abstract}

Index Terms - ITU, SAM 


\title{
A Novel Low-Power 5th order Analog to Digital Converter for Biomedical Applications
}

\author{
M.Aditya ${ }^{1}$ \\ ${ }^{1}$ Department of ECE, Koneru Lakshmaiah Education Foundation, \\ Green Fields, Vaddeswaram, A.P., India-522502. \\ ${ }^{1}$ E-mail ID: aditya@klunivesity.in
}

\begin{abstract}
:
This paper implements sigma delta analog to digital converters that play a key role in ECG acquisition. In this paper we have implemented both fifth and sixth order sigma delta analog to digital converters and compared the parameters like SNR, Nodal spectrum analysis, Integrated power analysis with respect to same frequency given to both fifth and sixth order circuits. In this paper we observed the increase in power consumption as the order increases. For 5th order Sigma Delta ADC, we observed SNR of $74.8532 \mathrm{~dB}$. Similarly for 6th order ADC the SNR observed was $75.5813 \mathrm{~dB}$
\end{abstract}

Index Terms - Delta, ADC, ECG, SNR, acquisition 


\title{
PV Variations of Pulsed Latch Circuits
}

\author{
M.Aditya ${ }^{1}$ \\ ${ }^{1}$ Department of ECE, Koneru Lakshmaiah Education Foundation, \\ Green Fields, Vaddeswaram, A.P., India-522502. \\ ${ }^{1}$ E-mail ID: aditya@klunivesity.in
}

\begin{abstract}
:
In this paper we studied the change in the result or action of different PV variations on the behaviour of Pulsed Latches by taking the reaction on both the Pulser and the Latch. Pulsed Latches are gaining reduced delay and power consumption in low power ASIC design technology. They provide another sequential element with high performance and low area and power consumption, taking advantage of both latchs and flip-flop features. This process implements the pulser by using MUX based pulser design. This design approaches are presented to improve the quality of Pulsed Latches circuits by using their main importance of high performance, low power, and small area. The proposed design has low power variation when operating at normal supply voltage.
\end{abstract}

Index Terms - Pulsed latches, variability, process variation, scaling of voltage, less power. 


\title{
Design Of Multiband Microstrip Patch Antenna For Wlan,Wimax And X-Band Applications
}

\author{
Pronami Bora ${ }^{1}$, Challapalliguna sekhar ${ }^{2}$, Phatan Sameer khan ${ }^{3}$, \\ Kokatam.vinay reddy ${ }^{4}$, Marthala.Venkat $\mathrm{sai}^{5}$, P.Pardhasaradhi ${ }^{6}$ \\ ${ }^{1}$ Department of ECE, Koneru Lakshmaiah Education Foundation, \\ Green Fields, Vaddeswaram, A.P., India-522502. \\ ${ }^{1}$ E-mail ID: pronamiz@gmail.com
}

\begin{abstract}
:
This letter describes a small, single-feed, circularly polarized patch antenna for broadband. The antenna consists of a microstrip patch and pins on the Reactive Impedance Surface (RIS) inspired by metamaterials. The RIS structure, consisting of 4 or 4 standard metallic square patches and four shorting pins which helps to improve the antenna bandwidth. The final optimized structure exhibits an impedance bandwidth of $53.75 \%(4.650 \mathrm{GHz}-7.617 \mathrm{GHz})$ along with a 3-dB axial ratio bandwidth of $30.4 \%(4.6242 \mathrm{GHz}-6.3036 \mathrm{GHz})$. Moreover, the proposed antenna yields a good broadside gain of $5.8 \mathrm{dBi}$ at $5.52 \mathrm{Ghz}$
\end{abstract}

Index Terms -Circular polarization, patch antenna, reactive impedance surface, broadband 


\title{
Metamaterial Loaded CSRR Based Antenna For WLAN And IOT BAND Applications
}

\author{
Pronami Bora ${ }^{1}$, Chayan Paul $^{2}$ \\ ${ }^{1}$ Department of ECE, Koneru Lakshmaiah Education Foundation, \\ Green Fields, Vaddeswaram, A.P., India-522502. \\ ${ }^{1}$ E-mail ID: pronamiz@gmail.com
}

\begin{abstract}
:
In this paper, we are dealing with a planar structure-based metamaterial inspired microstrip patch antenna using complementary split ring resonator [CSRR]. This microstrip patch antenna works in $\mathrm{C}$ band which can be resonated at operating frequencies ranging from $4.8 \mathrm{GHz}$ to $5.2 \mathrm{GHz}$, where it works in wireless local area network [WLAN] and IOT applications such as WIFI and WIMAX etc., In this antenna, the material used for the substrate is FR4 epoxy. This microstrip patch antenna is designed and analyzed then simulated using high frequency structure simulator [HFSS] software in the version 13.0.
\end{abstract}

Index Terms -CSRR, HFSS, IOT, Metamaterial, microstrip, WLAN, WIMAX. 


\title{
An Implementation of Futuristic Deep Learning Neural Network for Neurons Recognition in Proteomic and Genomic Payload Images
}

\author{
Aswin Kumer S V ${ }^{1}$, L S P Sairam Nadipalli ${ }^{2}$, P. Kanakaraja ${ }^{3}$ \\ ${ }^{1}$ Department of ECE, Koneru Lakshmaiah Education Foundation, \\ Green Fields, Vaddeswaram, A.P., India-522502. \\ ${ }^{1}$ E-mail ID: svaswin@gmail.com,sai,nadipalli@gmail.com, pamarthikanakaraja407@gmail.com
}

\begin{abstract}
:
The recognition of neurons in the images is the challenging task to find the behaviour of neurons and also, whether, the neurons are alive or not. The neuron helps to convey the information to the brain in the human body. Based on the actions and emotions of the human being, the count of neurons will be varied. First, the neurons in an image should be detected, then the alive status of neurons can be easily found out using the Deep learning neural network. In this proposed approach, the deep learning neural network is used to detect the count of neurons as well as the live status of neurons. The image consists of proteomic and genomic payload is considered as the input image for this implementation. The trained deep neural network has many hidden layers for improving accuracy to detect the live status of neurons. To perform this, the Raspberry Pi development board is used. The neuron image is fetched from the cloud database and processing those images and the output of the evaluation will be again stored in the same cloud database. The images in the cloud database is in the form of datasets or binary image which can be converted into actual digital image with the help of programming the Raspberry Pi development board.
\end{abstract}

Index Terms - Binary Image, Image Classifier, Deep Neural Network, Cloud Database, Raspberry Pi, Behaviour of Brain Neurons 


\title{
Volcano Monitoring System using Drone-Bot and IoT
}

\author{
Aswin Kumer S V ${ }^{1}$, L S P Sairam Nadipalli ${ }^{2}$, P. Kanakaraja ${ }^{3}$ \\ ${ }^{1}$ Department of ECE, Koneru Lakshmaiah Education Foundation, \\ Green Fields, Vaddeswaram, A.P., India-522502. \\ ${ }^{1}$ E-mail ID: svaswin@gmail.com, sainadipalli@gmail.com, pamarthikanakaraja407@gmail.com
}

\begin{abstract}
:
Volcanoes can hit the sky and reach the ground surface at any time in some areas and it is very difficult to monitor time to time. Human beings cannot check the live status of volcano in that areas because of the huge temperature. Instead of sending human beings to that place, Drone-Bot is used to measure the live lava temperature and it alerts the ground station to protect people near the surroundings. The thermocouple is used as a temperature sensor which can measure the wide range of higher temperature and it can be interfaced with the TTGo T-Call development board to process and send the temperature data to the ground station through GSM as Short Message Service (SMS), also the ESP-32 CAM is interfaced with that development board to capture the snapshot of the mountain if the temperature is high and the same snap is shared to the ground station through Wi-Fi. The GPS module is also interfaced with the development board to know the location of the volcano happening mountain. In that reserved area, the GSM signal and Wi-Fi signal is not available. So, the development board stores those data in the EPROM and the same data is communicated when the Drone-Bot reaches the signal communication zone. The DroneBot charging station is also available in that same zone. All the data are stored and monitored in the cloud database.
\end{abstract}

Index Terms -TTGo T-Call, ESP-32 CAM, Thermocouple Sensor, GSM, GPRS, Global Positioning System (GPS), Drone-Bot, Electronic speed controller (ESC), Brushless DC Motor $(B L D C)$ 


\title{
Structural and optical properties of Fe doped Ti02Nanoparticles for Efficient photocatalytic Applications
}

\author{
S. Arunmetha ${ }^{1}$, and R. Jayavel ${ }^{2}$ \\ ${ }^{1}$ Department of ECE, Koneru Lakshmaiah Education Foundation, \\ Green Fields, Vaddeswaram, A.P., India-522502. \\ ${ }^{2}$ Crystal Growth Centre, Anna University, \\ Chennai-600 025, Tamil Nadu, India \\ E-mail: sarunmetha@kluniversity.in.
}

\begin{abstract}
:
The present work focuses on the synthesis, characterization and photocatalytic activity of a nanosized Fe-doped $\mathrm{TiO}_{2}$ photocatalyst. Sequences of Fe-doped $\mathrm{TiO}_{2}$ samples were prepared using a sol-gel method. The Fe-doped $\mathrm{TiO}_{2}$ the existing states of $\mathrm{Fe}^{3+}$, the transformation of existing states and the phase transition, optoelectronic and magnetic properties are investigated. The prepared materials are investigated by using techniques such as X-ray diffraction (XRD), scanning electron microscopy (SEM), transmission electron microscopy (TEM), X-ray photoelectron spectroscopy (XPS), UV-visible spectroscopy, photoluminescence (PL) spectroscopy, Raman spectroscopy. The synthesized samples are pure and crystalline in nature and show a tetragonal phase of $\mathrm{Fe}_{2} \mathrm{TiO}_{5}$ and Raman spectroscopy also confirmed the formation of a tetragonal phase structure. The observed results revealed that the existing states of $\mathrm{Fe}^{3+}$ and the transformation of existing states $\left(\mathrm{Fe}^{3+}, \mathrm{Fe}_{2} \mathrm{O}_{3}, \mathrm{FeTiO}_{3}\right.$ and $\left.\mathrm{Fe}_{2} \mathrm{TiO}_{5}\right)$ are closely related to the concentration $\mathrm{Fe}$ and transition is promoted by the substitutional doped $\mathrm{Fe}$ in the $\mathrm{TiO}_{2}$ lattice. The spectra from the DRS showed a red shift and a reduction of $3.2 \mathrm{eV}$ to $2.4 \mathrm{eV}$ in the band gap energy for Fe-doped $\mathrm{TiO}_{2}$. Meanwhile the photocatalytic activity of the materials is increased. The Fe-doped $\mathrm{TiO}_{2}$ nanoparticles is improves the optical and electrical properties of $\mathrm{TiO}_{2}$ nanoparticles. This work may be useful in understanding the doping of Fe-doped $\mathrm{TiO}_{2}$, and fabrication of photocatalytic materials.
\end{abstract}

Index Terms -Fe-doped $\mathrm{TiO}_{2}$ nanoparticles, structural properties, optical properties, Photocatalytic activity. 


\title{
Metal and non-Metal doped TiO2 Nanoparticles for Enhanced Photovoltaic Device Applications
}

\author{
S. Arunmetha ${ }^{1}$, and R. Jayavel ${ }^{2}$ \\ ${ }^{1}$ Department of ECE, Koneru Lakshmaiah Education Foundation, \\ Green Fields, Vaddeswaram, A.P., India-522502. \\ ${ }^{2}$ Crystal Growth Centre, Anna University, \\ Chennai-600 025, Tamil Nadu, India \\ E-mail: sarunmetha@kluniversity.in.
}

\begin{abstract}
:
The development of alternative, economically competitive strategies to produce solar fuels is of strong interest. The most efficient photocatalytic and electrocatalytic materials are noble metals, which are prohibitively expensive, and the abundant materials are not fully developed up to their potential performance. The challenges have been identified the proposed materials should meet the following criteria in order to be viable photoelectrode in general; (i) can absorb ample visible light in solar spectrum (a proper band gap), (ii) comprise earth-abundant elements. To meet this requirement, the metal and non-metal doped titania nanomaterials having sustainable applications in photocatalytic and photo electrochemical area. The synthesis of metal (Fe) and non-metal (S) doped nanostructure titania. In research work nanostructured $\mathrm{S}-\mathrm{TiO}_{2}$ and $\mathrm{FeTiO}_{3}$ were formed using simple chemical method and identify new promising materials for energy conversion process. The physico-chemical and optical properties were analyzed. It has been identified that the prepared nanostructure materials work well as a photoanode for the DSSCs, the device has a favorable band gap from UV to visible region. The comparative potential research work also continues towards the design and construction of a pilot plant that will be capable of producing materials and systems with practicability and scalability. Demonstrates promising opportunities and more requirements are reducing the cost for improving efficiency in practical applications.
\end{abstract}

Index Terms - Metal and non-metal doped $\mathrm{TiO}_{2}$ nanoparticles, physicochemical properties, optical properties, Photovoltaic Device. 


\title{
Inner-Gate-Engineered GAA MOSFET to Enhance the Electrostatic Integrity
}

\author{
Biswajit Jena ${ }^{1}$, Sidhartha Dash ${ }^{2}$, Soumya Ranjan Routray ${ }^{3}$, Guru Prasad Mishra \\ ${ }^{1}$ Department of Electronics and Communication Engineering Koneru Lakshmaiah Education \\ Foundation (K L Deemed to be University), Vaddeswaram Guntur 522502 Andhra Pradesh, \\ ${ }^{2}$ Department of Electronics and Communication Engineering Institute of Technical Education \\ and Research Siksha 'O' Anusandhan Deemed to be University Khandagiri, Bhubaneswar \\ 751030, Odisha \\ ${ }^{3}$ Department of Electronics and Communication Engineering SRM Institute of Science and \\ Technology, Kattankulathur Chennai 603203, Tamil Nadu \\ ${ }^{4}$ Department of Electronics and Communication Engineering National Institute of Technology \\ Raipur, Raipur 492010, Chattisgarh \\ ${ }^{1}$ E-mail ID: biswajit18590@gmail.com
}

\begin{abstract}
:
Gate-all-around (GAA) MOSFETs are the best multi-gate MOSFET structure due to their strong electrostatic control over the channel. The electrostatic controllability can be enhanced further by applying some gate engineering technique to the existing GAA structure. This paper investigates the effect of inner gate (core gate) on the electrostatic performance of conventional GAA MOSFET. The inner gate engineering increases both the electrostatic control and packing density of GAA MOSFET. In this paper, we have presented an inner-gate-engineered (IGE) GAA MOSFET and inspected its advantages over conventional counterparts. The proposed structure exhibits higher Ion/Ioff ratio, low threshold voltage and improved RF performances as compared to the conventional structure. Analytic simulation has been carried out for numerous figures of merit (FOMs) for different technology nodes.
\end{abstract}

Index Terms - Gate-all-around (GAA), inner-gate-engineered (IGE)figures of merit (FOMs)radio frequency $(\mathrm{RF})$ 


\title{
Performance Analysis of GAA MOSFET for Lower Technology Nodes
}

\author{
P.Keerthana ${ }^{1}$, P. Praneeth Babu' ${ }^{2}$ T.Akhil Babu ${ }^{3}$ and B. Jena ${ }^{4}$ \\ ${ }^{1}$ Department of ECE, Koneru Lakshmaiah Education Foundation, \\ Green Fields, Vaddeswaram, A.P., India-522502. \\ ${ }^{1}$ E-mail ID: biswajit18590@gmail.com
}

\begin{abstract}
:
With the continuous down scaling of transistor size in order to improve the packing density, pushing the transistor design into different level. Starting from planar structure to complex 3-D nanoelectronics devices, the device performance changes drastically as the miniaturization of device dimension occurs to attain current need of the semiconductor industry. In this work the performance analysis of different Figure of merits (FOMs) are studied extensively for lower technology nodes. The transfer and output characteristics are provided with different gate and drain bias to study the sub-threshold and short channel effects. Apart from that some simulator generated structures are provided to visualize the surface potential and electric field completely. The superiority of Gate All Around (GAA) MOSFET over other conventional MOSFET in Nano scale paving a path for next generation transistor scaling.
\end{abstract}

Index Terms - GAA, MOSFET, Nano, Scaling, Sub-threshold 


\title{
An Optimal Hybrid Solution to Local and Global Facial Recognition Through Machine Learning
}

\author{
K. Raju ${ }^{1}$, B. Chinna rao ${ }^{2}$, N.L. Pratap ${ }^{3}$ \\ ${ }^{1}$ Department of ECE, Narasaraopeta Engineering College, Narasaraopet, Guntur, AP, India. \\ ${ }^{2}$ Department of ECE, NS Raju Institute of Technology, Visakhapatnam, India. \\ profbcrao@gmail.com \\ ${ }^{3}$ Nalajala Lakshman Pratap, Asst Prof, Department of Electronics and Communication \\ Engineering, Koneru Lakshmaiah Education Foundation, Vaddeswaram, AP, India. \\ Email:lakshmanpratap@kluniversity.in
}

\begin{abstract}
:
Face recognition need is fine assured as enormous industrial relevance to use them toimplement one or another objective. As facial recognition has beating advantages over other industrial applications and human eyes can easily evaluate performance, improved algorithms and smaller computing costs are continuously improving this methodology. This research takes the conventional algorithms of recognition in the first stage and use hybrid approaches to counter their limitations. The research starts with basic computation of global face features using Principal Component Analysis, Discrete Wavelet Transform and Independent Component Analysis, with some standard NN classifiers and Support Vector Machine. It has observed that as learning rate are high in machine learning then accuracy of system goes high but increases the area and cost overhead. To overcome that training limitation, a Fusion based methods has been proposed in further work, based on Harris corner, Speed Up Robust Features and DWT+PCA system model where only $10 \%$ training sample has been taken on Essex database and $99.45 \%$ accuracy is achieved. To overcome limitations further an efficient Hybrid method proposed which elaborates the local features of face. Further these features trained with Neural Network classifier to obtained better accuracy nearly $99.40 \%$ with single image training from each class.
\end{abstract}

Index Terms - DWT, PCA, ICA, LBP, HOG, GLCM, HARRIS CORNER. 


\title{
Data Security in Cloud Computing Using Three-Factor Authentication
}

\author{
Sunanda Nalajala ${ }^{1}$, B. Moukthika ${ }^{1}$, M. Kaivalya ${ }^{1}$, N.L. Pratap ${ }^{2}$ \\ ${ }^{1}$ Department of CSE, Koneru Lakshmaiah Education Foundation, \\ Green Fields, Vaddeswaram, A.P., India-522502. \\ E-mail: sunanda@kluniversity.in. \\ ${ }^{2}$ Department of Electronics and Communication Engineering, Koneru Lakshmaiah Education \\ Foundation, Green Fields, Vaddeswaram, A.P., India-522502 \\ E-mail: lakshmanpratap@kluniversity.in
}

\begin{abstract}
:
Cloud storage is tremendously increasing its services, huge range of storage and communication of massive data over network. This practically has an adverse effect on the way of upholding this data when it especially comes to the privacy of the user-security. We first view you with a system that is vulnerable to this authentication protocol with its misuse of biometrics and incorrect password generates no user to lose the mobile device. We even went along with this scheme and gave out a major issue to overcome with impersonation attack. However, this scheme makes the way easy to attack for offline password guessing attack. We included a three-factor authentication which carries the smart card into card reader, gets the password and identity and conveys the user details' requesting time. We then came up with a scheme to overcome these security flaws of this prescribed authentication scheme combining passwords, mobile devices, and biometrics. The proposed system is robust three-factor authentication with the help of password, biometrics and mobile device which provides reliable security strength to the user's data and makes counterattack to existing attack, giving with more benefits compared to the previous scheme. This scheme provides with most enhanced security functionalities.
\end{abstract}

Index Terms -Three-factor authentication Counter attacks Privacy Biometrics Smart cards 


\title{
A novel approach in optimal parameters selection for high performance and ultra-low power $\Sigma \Delta$ Modulator
}

\author{
S. Vamsee Krishna ${ }^{1}$ M. Lakshmana Kumar ${ }^{2}$ and R. S. Ernest Ravindran ${ }^{3}$ \\ ${ }^{123}$ Department of ECE, Koneru Lakshmaiah Education Foundation, \\ Green Fields, Vaddeswaram, A.P., India-522502. \\ E-mail: somavrapuvamseek@ kluniversity.in
}

\begin{abstract}
:
A linear model analysis is made for second to fourth order cascaded, single loop and hybrid sigma delta modulator architectures with a novel technique for deriving choice of parameters. Parameters obtained in this paper by considering the trade-offs between architectural to physical level implementation in top-down design approach, which guides efficient practical implementation of sigma delta modulator. With the proposed choice of parameters, the performance metrics of sigma delta modulators are verified in behavioural modelling and comparison of selected architectures are given for optimum selection of circuit topology and modulator parameters in design of SigmaDelta analog to digital converters.
\end{abstract}

Index terms: Sigma delta modulator, top-down design, behavioural modelling. 


\title{
Systematic design and analysis of 3rd order single loop $\Sigma \Delta$ Modulator for ECG signal processing
}

\author{
S. Vamsee Krishna ${ }^{1}$ M. Lakshmana Kumar ${ }^{2}$ and R. S. Ernest Ravindran ${ }^{3}$ \\ ${ }^{123}$ Department of ECE, Koneru Lakshmaiah Education Foundation, \\ Green Fields, Vaddeswaram, A.P., India-522502. \\ E-mail: somavrapuvamseek@kluniversity.in
}

\begin{abstract}
:
This paper presents system level modelling of sigma delta modulator including circuit level non idealities in MATLAB/Simulink environment for low frequency applications. An overview of sigma-delta modulation technique in analog-to-digital conversion and implementation of $\sum \Delta$ analog-to- digital converters using different technology processes, architectural and circuit level methods explored. A third order single loop sigma delta modulator is designed and verified with careful selection of integrator weights to meet stable desired performance. The proposed designed achieved SNDR of 101.75dB and 17-bit resolution. The simulation performed at behavioural level using Simulink based sigma delta simulator, the results obtained are accurate, by considering non ideal effects. The results obtained for the proposed cascaded sigma delta modulator compared with existing single loop and cascaded architectures and proved as efficient.
\end{abstract}

Index terms: analog-to-digital conversion, $\Sigma \Delta$ modulator, CMOS. 


\title{
Effect of Moderate Atomic Mass Ions on Inas/Gaas Quantum Dots
}

\author{
S. Upadhyay ${ }^{1}$ \\ ${ }^{1}$ Department of ECE, Koneru Lakshmaiah Education Foundation, \\ Green Fields, Vaddeswaram, A.P., India-522502. \\ E-mail ID: sourabh@kluniversity.in
}

\begin{abstract}
:
All investigated QDs were grown through MBE on semi-insulating GaAs (100) substrate. Prior to the growth, GaAs substrates were thermally annealed at $610^{\circ} \mathrm{C}$ to desorb the protective oxide layer under high vacuum. Next, a 200 -nm GaAs buffer layer was deposited at $590^{\circ} \mathrm{C}$, following which a 50-nm GaAs active layer was grown. Subsequently, the substrate temperature was ramped down to $500^{\circ} \mathrm{C}$, and 2.7-ML InAs was deposited at a growth rate of $0.1 \mathrm{ML} / \mathrm{s}$. The first few layers of InAs contribute to the formation of the wetting layer, which is lattice matched to the substrate up to a certain thickness $(1.8 \mathrm{ML})$; beyond this threshold thickness, the strain energy relaxes, resulting in the formation of self-assembled QDs. Subsequently, the QDs were capped with a 50-nm GaAs layer for carrier confinement, atop which a similar 2.7-ML InAs layer was grown to study QD morphology.The implanted QDIP exhibited suppressed dark current density compared to the as grown devices.
\end{abstract}

KEY-WORDS: Gaas, Moderate, QD morphology 


\title{
High-Energy Proton Implantation of In (Ga)As/Gaas Quantum-Dot Heterostructures
}

\author{
S. Upadhyay ${ }^{1}$ \\ ${ }^{1}$ Department of ECE, Koneru Lakshmaiah Education Foundation, \\ Green Fields, Vaddeswaram, A.P., India-522502. \\ E-mail ID: sourabh@kluniversity.in
}

\begin{abstract}
:
We studied the optical properties of the implanted samples through low-temperature (15 K) PL spectroscopy. Carriers were optically excited using a 2.33-eV green laser as the source of energy; this energy is higher than the bandgap of the barrier layer (GaAs). PL emission from the as-grown sample exhibited a typical Gaussian profile, with a shoulder peak at a lower wavelength. To verify the nature of the shoulder peak, power-dependent PL spectra were measured by varying the laser power density over the range $22 \mathrm{~mW} / \mathrm{cm} 2$ to $1.1 \mathrm{~kW} / \mathrm{cm} 2$. We derived the thermal activation energies from temperature-dependent PL plots and observed a reduction in the activation energies of all implanted samples, suggesting that reduced carrier confinement due to the creation of additional defects. We further verified these results through HRXRD and found that the crystalline quality of the samples degraded on implantation, as evidenced by the increasing disappearance of the satellite peaks with increase in the ion dose.
\end{abstract}

KEY- WORDS: Gaas, HRXRD, Crystalline 


\title{
2-bit Vedic Multiplier Design Using Urdhva- Tiryagbhyam and Modified-Gate Diffusion Input Technique
}

\section{NgangbamPhalguni Singh ${ }^{1}$, Nadendla Bhargava Sai ${ }^{1}$, Venna Venkat Kumar ${ }^{1}$, Cheruvu Krishna Chaitanya ${ }^{1}$}

\author{
Department of Electronics and Communication Engineering, Koneru Lakshmaiah Education \\ Foundation, Vaddeswaram, A.P., India-522502. \\ E-mail ID: npsingh@kluniversity.in,
}

\begin{abstract}
:
This paper presents a Vedic Multiplier design which are designed by adopting Modified Vedic Mathematics sutra called Urdhva-Tiryagbhyam. The proposed Vedic multiplier scales down the number of steps required to obtain the final product, thereby reducing the delay of the circuit. A high-speed multiplier depends mainly on adder block performance. Improving adder block performance, the total energy consumption is reduced by $28 \%$ and delay factors of the augmented multiplier module are the main focal points of this paper. Thus the speed of the multiplication is increased by $30 \%$. The proposed 2-bit Vedic Multiplier circuit design using Modified-Gate Diffusion Input (GDI) Technique is used as a primary element in configuring the structure of higher order Vedic multiplier circuits. Modified-GDI Technique overcomes all the drawbacks of original GDI cell such voltage swing reduction caused due to threshold drops which leads to increase in static power dissipation and performance reduction. This design of 2-bit Vedic Multiplier is done using Mentor Graphics in the CMOS $130 \mathrm{~nm}$ technology.
\end{abstract}

Index Terms: Vedic Multiplier, Urdhva-Tiryagbhyam, Mentor Graphics, Modified-GDI Technique. 


\title{
Approximate Sum-of-Products Design Based on Quantum- Dot Cellular Automata Using Distributed Arithmetic
}

\section{NgangbamPhalguni Singh ${ }^{1}$, Putta Lakshmi Ganesh ${ }^{1}$, Aluru Venkata Sai Krishna $^{1}$, Madala Bramhendra ${ }^{1}$}

${ }^{1}$ Department of Electronics and Communication Engineering, Koneru Lakshmaiah Education Foundation, Vaddeswaram, A.P., India-522502.

Emails: npsingh@kluniversity.in, lakshmiganesh199808@gmail.com, alurusaikrishna@gmail.com, bramhendra621@gmail.com

\begin{abstract}
:
This paper presents 16-bit improved Approximate Sum-of-Products (ASOP) which is designed to achieve better results in fields of area and delay. This approximate circuit helps in improving performance of the Distributed Arithmetic operations with reduced area and delay. Sum-ofProducts circuits plays an important role in many digital signal processing applications. This proposed model is validated by utilizing Quantum-Dot Cellular Automata (QCA) method in Distributed Arithmetic operations. Approximate computing is used for improving the performances of the design. The proposed design shows improvement of $(68.66-83.22) \%$ in area. It is also observed that the proposed model achieves $11.11 \%$ improvement in delay.
\end{abstract}

Index Terms - Approximate Sum-of-Products (SOP), Distributed Arithmetic, Quantum-Dot Cellular Automata (QCA), Approximate computing. 


\title{
TRANSITION ANALYSIS OF BEAM SCINTILLATION IN R-FSOC LINKS USING DISCRETE MARKOV CHAIN MODEL
}

\author{
Rahul Bose ${ }^{1}$ \\ ${ }^{1}$ Department of ECE, Koneru Lakshmaiah Education Foundation, \\ Green Fields, Vaddeswaram, A.P., India-522502. \\ E-mail ID: rahulbose@kluniversity.in
}

\begin{abstract}
:
Among the various sources of the beam fading in reflector-assisted FSOC links, the impact of the atmospheric turbulence is deleterious as it randomly alters the power distribution profile of the traversing optical beam. Consequently, this paper presents an instantaneous CSI estimation technique from the impinging scintillated beam and is developed based on the asymptotic solutions generated by the perturbation techniques. The discussed CSI technique can gauge the strength of the optical scintillation in an R-FSOC link with turbulent channel which is characterized by the LN distribution. This paper analyzes the reliability of the propounded CSI estimation scheme in a non-uniform temperature inflicted turbulent channel which is modeled by Gamma-Gamma (GG) distribution. The paper then continues with the statistical analysis of the proposed system performance in terms of the average BER and investigation of the scintillation transition probability matrix of the underlying turbulent channel. The Eigen analysis of the statistically generated transition probability matrix is then adopted for evaluating the limiting transition probability of the beam scintillation.
\end{abstract}

Keywords: Free space optics, Infrared links, Laser beams, Markov processes, Optical communication, Reflection, Scintillation 


\title{
Bio-sensing with Enhanced Sensitivity by Noise Spectroscopy Analysis
}

\author{
Naren Das ${ }^{1}$ \\ ${ }^{1}$ Department of ECE, Koneru Lakshmaiah Education Foundation, \\ Green Fields, Vaddeswaram, A.P., India-522502. \\ Email: narendas@ kluniversity.in
}

\begin{abstract}
:
Biosensors with ordered nanopores structure have been demonstrated as highly sensitive and selective platform for label free electrical detection of biomolecules through unique frequency dependent sensitivity characteristics. These sensors have been found to be capable of detecting down to very low concentration ( $1 \mathrm{fg} / \mathrm{ml}$ ) of Hep-B surface antigen in blood without the requirement of any centrifuge or pre-concentration. To further lower the detection limit, recently, the design considerations of such sensors have been explored to improve their performance and it has been observed that the optimized pore geometry imparts the best combination of sensitivity, selectivity and repeatability. However, the steady state impedance characteristics is likely to drift in the presence of physiological solution like blood since the various ions can contaminate the silicon oxide surface and pore walls. This will deteriorate the performance even for optimized sensors and hence achieving sub-femtomolar detection limit will be difficult. Under such circumstances, noise spectroscopy analysis can be employed since the measured electrical signal is usually submerged in noise arising from the random movement of ions at the interfaces, the dynamics of the antigen-antibody binding and the intrinsic device noise owing to carrier fluctuations. With this view, nanoporous silicon oxide immune sensors have been fabricated and both the steady state impedance response and the noise spectroscopy behaviour have been measured to demonstrate the level of detection of Hep-B virus in blood. The amplitude and cutoff frequency have been extracted from the noise power spectral density (PSD). Further, the specificity of detection has been verified with Hep-C spiked in blood.
\end{abstract}

Index Terms: Nanoporous silicon, Noise spectroscopy, Immunosensor 


\title{
A Low Power Auto Adaption Unit for Reconfigurable Data Converter
}

\author{
K. Hari Kishore ${ }^{1}$ \\ ${ }^{1}$ Department of ECE, Koneru Lakshmaiah Education Foundation, \\ Green Fields, Vaddeswaram, A.P., India-522502. \\ Email: kakarla.harikishore@kluniversity.in
}

\begin{abstract}
:
This paper substantiates a simple auto adaption unit that is used for Reconfigurable ADC which is suitable for biomedical and mixed signal circuit test applications. The adaption unit make available in order to configure the analog to digital converter (ADC) resolution and the sampling rate regardless of the converter circuit. It generates control bits which controls reconfigurable ADC to operate on various modes based on the frequency of the input signal and the amplitude. The paper utilized models of FVC and Schmitt trigger concepts to design auto adaptation unit. The adaption unit consumes $106.2 \mu \mathrm{W}$ with a supply voltage of $1 \mathrm{~V}$. The entire circuitry is designed, simulated and analysed by using Cadence EDA tool with $180 \mathrm{~nm}$ CMOStechnology.
\end{abstract}

Keywords: Adaptation unit, Frequency to voltage converters,reconfiguration 


\title{
Design of Low Power, Low Voltage Single-Phase Clocking Flip-Flops for Sequential Circuits
}

\author{
K. Hari Kishore ${ }^{1}$ \\ ${ }^{1}$ Department of ECE, Koneru Lakshmaiah Education Foundation, \\ Green Fields, Vaddeswaram, A.P., India-522502. \\ Email: kakarla.harikishore@kluniversity.in
}

\begin{abstract}
:
The basic element in sequential circuit design is flip-flop and flip-flops are widely used in memories. This paper outlines the design of Single-Phase Clocking flip-flop using various methods like pass transistor and the transmission gate logic. The main objective of this paper is to design a flip-flop with optimized power. The optimization of power and the reduction in transistor count is achieved by using transmission gates and pass transistor logic. The performance metrics of Flipflop designs are compared with different sequential circuits using transmission gate and pass transistor logic techniques. The results show that the proposed structures exceeded expectations towards optimizing the average power. When compared with the other designs, the proposed flipflop is designed using only 11-transistors and the average power consumption of the proposed flipflop is bring downed to $108.9 \mathrm{nW}$. All the designs are simulated and verified with PYXIS tool running on $3.41 \mathrm{GHz}$ processor. These flip-flop designs are best suited for low power and highperformance applications.
\end{abstract}

Keywords: Transmission Gate, Mater Slave Flip-flop, Power Consumption, True Single-Phase Clock 


\title{
A Compact triple notch band CPW-fed Flexible antenna for UWB applications
}

\author{
B. T. P. Madhav ${ }^{1}$ \\ ${ }^{1}$ Professor, Department of ECE, Koneru Lakshmaiah Education Foundation, \\ Green Fields, Vaddeswaram, A.P., India-522502. \\ E-mail: btpmadhav@kluniversity.in
}

\begin{abstract}
:
This article presents a compact elliptical-shaped flexible antenna with CPW (coplanar waveguide) feeding method and is designed to attain triple notch band characteristics. To achieve the UWB frequencies, the radiating element is designed in an elliptical shape and was fed by the slotted CPW feed line. This proposed design has a $166.19 \%$ fractional bandwidth with a $7.1 \mathrm{GHz}$ center frequency in the simulation, and a decent match has been observed in the measurement through the values of $170.10 \%$ bandwidth with a $7.025 \mathrm{GHz}$ center frequency. The defected ground structure (DGS) and circular shaped split-ring resonators (SRRs) are responsible for attaining the triple notch bands. The first notch band (2.0-2.70 GHz) is achieved by placing a circular SRR on the patch element, and the notch is engaged for the band rejection of WLAN (2.4 $\mathrm{GHz}$ ). Another circular SRR in the patch is responsible for the second notch (3.45-3.80) and is involved in the migration of the interference caused by the WiMAX system of $3.5 \mathrm{GHz}$. The third notch (5.15-6.20) is achieved by the DGS structure and is assigned for suppressing the interference of WLAN systems of 5.2/5.8 GHz and WiMAX systems of 5.5 GHz. The proposed antenna has shown robust performance in different bending circumstances and also suitable for operating it in curved UWB systems.
\end{abstract}

Index Terms -cpw, monopole antenna, DGS, ultra-wideband. 


\title{
A liquid crystal polymer substrate-based frequency reconfigurable monopole antenna for vehicular communication applications.
}

\author{
B. T. P. Madhav ${ }^{1}$ \\ ${ }^{1}$ Professor, Department of ECE, Koneru Lakshmaiah Education Foundation, \\ Green Fields, Vaddeswaram, A.P., India-522502. \\ E-mail: btpmadhav@kluniversity.in
}

\begin{abstract}
In this article, an ultra-thin and compact frequency reconfigurable antenna is developed on a liquid crystal polymer substrate. The proposed antenna is intended for applications in vehicular communications with a compact size of $40 \times 30 \times 0.1 \mathrm{~mm}^{3}$. The proposed antenna is fabricated on a Rogers ULTRALAM-3850 liquid crystal polymer with a Er value of 2.9; the antenna radiating elements contains a circular ring structure fed by a CPW feeding mechanism. The proposed antenna resonates in the frequencies of $2.4 \mathrm{GHz}, 5.9 \mathrm{GHz}, 9.5 \mathrm{GHz}$, and $12.5 \mathrm{GHz}$. The reconfigurability is achieved by incorporating two BAR 64-03W PIN diodes as switches. Furthermore, this study also analyzed the effects of radomes permittivity and its housing effects on the proposed antenna's different switching configurations. Far-field characteristics are also analyzed by placing the antenna enclosed by the radome on the vehicle's rooftop. Finally, to investigate the radome paint effects on the signal, experimentation is made on sensing the strength of the receiving signal in the real-time environment. The proposed antennas gain in the standalone condition in all the resonating frequencies vary from -0.51 to $6.12 \mathrm{~dB}$ ), and with the radome, its values diverge from 2.85 to $8.54 \mathrm{~dB}$.
\end{abstract}

Index Terms - reconfigurable antenna, PIN diodes, liquid crystal polymer. 


\title{
Cantilever based MEMS sensor for agricultural applications
}

\author{
N. Siddaiah ${ }^{1}$, V.V. Praveen $^{1}$, P. KavyaSri ${ }^{1}$ \\ ${ }^{1}$ Department of ECE, Koneru Lakshmaiah Education Foundation, \\ Green Fields, Vaddeswaram, A.P., India-522502. \\ E-mail: nalluri.siddu@kluniversity.in.
}

\begin{abstract}
:
Detection of gases using MEMS sensors are discussed in this paper. Metal oxide semiconductorbased gas sensors are designed and modeled using various cantilever structures. Cylindrical and rectangular type cantilevers were used and simulated using modelling tool and different results were compared. The design sensor has wide range of applications and high resolution in gas sensing. Cantilever with $15 \mu \mathrm{m}$ of width was used as sensing layer on Platinum micro heater without coil and this arrangement provides better heating and takes less time to heat the sensor. The sensor was designed using metal oxide semiconductor technology and for best physical architecture.
\end{abstract}

Index Terms - Cantilever, $\mathrm{CO}_{2}$, gas sensor, metal oxide, conductivity, heat capacity, Thermal energy 


\title{
Design and analysis of RF MEMS switch for wireless
}

\section{communications}

\author{
N. Siddaiah ${ }^{1}$,B. Sri Keerthana $\operatorname{Reddy}^{1}$, B. Manoj Kumar ${ }^{1}$ \\ ${ }^{1}$ Department of ECE, Koneru Lakshmaiah Education Foundation, \\ Green Fields, Vaddeswaram, A.P., India-522502. \\ E-mail: nalluri.siddu@ kluniversity.in.
}

\begin{abstract}
:
Microelectromechanical systems are popularly known as MEMS and it is a system integrating several streams like electronics, electrical and mechanical on common substrate to develop a device. The MEMS devices combines the functionality of electronics, electrical and mechanical devices. Different elements are integrated in MEMS devices so that MEMS sensors can sense various parameters and controls the environment at the same time. The designed RF MEMS switch can work with low actuation voltage of $20 \mathrm{~V}$ and it was obtained from electromechanical analysis of switch. The capacitance ratio of 160 was obtained from proposed MEMS switch. Electromagnetic and electromechanical analysis was performed on proposed switch.
\end{abstract}

Index Terms - RF switch, low pull-in voltage, capacitance ratio, return loss, insertion loss, response time. 


\title{
Wind Energy Assessment over Andhra Pradesh using high resolution data
}

\author{
G. Ch. Satyanarayana ${ }^{1}$ and K. Siva Sankar ${ }^{2}$ \\ ${ }^{1}$ Department of ECE, Center for Atmospheric Science, Koneru Lakshmaiah Education \\ Foundation, Vaddeswaram, A.P., India-522502. \\ ${ }^{2}$ Andhra Pradesh State Development Planning Society, Andhra Pradesh, India \\ Email: gcsatya@kluniversity.in
}

\begin{abstract}
:
The socioeconomic welfare of any country is dependent on its sustainable development and harmony with nature. The use of sustainable resources such as wind, which is a clean energy being an imperative source of energy in the long run, contributes to long term ecological balance conserving the non-renewable resources. This study investigates the long term climatologicalmean wind speed distribution and provides an assessment of wind energy potential for a part of the Indian subcontinent. The wind data at $10-\mathrm{m}$ level at $12.5 \mathrm{~km}$ high resolution for the period $1979-2018$ is used. The present study confined to the regions of Andhra Pradesh; wind energy potential has been assessed for all the 13 districts of AP state. It is explicit that the wind speed varies remarkably from one location to another depending upon the terrain characteristics and seasonal atmospheric circulation features. The work reviews the degree of the consistency of the wind speed throughout the 40-year period as monthly, seasonally and annually. ERA-Interim wind data set describes the inordinate predominance of variations in the variability observed for each location. The potentiality of the wind energy is ferreted out significantly using Weibull probability density function, which provides a better approximation of wind energy assessment. The study further provides wind speed above $4.5 \mathrm{~m} / \mathrm{s}$ hours/year frequencies at each grid of the study region. Wind energy potential has also been estimated at four different levels below 120 meters and the optimised height for wind harness as $80-\mathrm{m}$ level is identified.
\end{abstract}

Key words: wind energy, wind power potential, spatial distribution, Weibull distribution 


\title{
Time-Frequency Analysis for Enhanced Subsurface Detail Visualization in Non-stationary Thermal Wave Imaging
}

\author{
V. Gopi Tilak ${ }^{1}$, V. S. Ghali ${ }^{1}$ and A. Vijaya Lakshmi ${ }^{1}$ \\ ${ }^{1}$ Infrared Imaging Center, Koneru Lakshmaiah Educational Foundation, Vaddeswaram, Andhra \\ Pradesh, India-522502. \\ E-mail.ID: gvs0raos@gmail.com
}

\begin{abstract}
:
Non-stationary optical stimulus-based infrared non-destructive testing modality has gained much attention from past decades. In the recent past, non-stationary thermal wave imaging (NSTWI) is advanced with various fascinating post-processing techniques. The time-domain analysis of pulse compression based correlation analysis evolved as a promising post-processing approach for NSTWI. On the other hand, time-frequency analysis based signal processing techniques became prominent processing techniques for non-stationary signal analysis. This article proposed a fanchirp transform-based time-frequency analysis technique in NSTWI as a post-processing technique and validated with the experimentation carried over a mild steel sample with flat bottom holes as subsurface anomalies. Defect signal to noise ratio and size estimation concerned as figures of merits to validate the proposed methodology. A comparative analysis with conventional processing techniques such as Fourier transform-based phase analysis and pulse compression suggested that proposed time-frequency analysis presents enhanced defect detection.
\end{abstract}

Index Terms:Non-stationary thermal wave imaging, pulse compression, fan chirp transform, Time-frequency analysis. 


\title{
Pulse Compression using Fractional Fourier Transform for Enhanced Depth Resolution in Quadratic Frequency Modulated Thermal Wave Imaging
}

\author{
V. Gopi Tilak ${ }^{1}$, Sk. Subhani ${ }^{1}$, V. S. Ghali ${ }^{1}$, A. Vijaya Lakshmi ${ }^{1}$ and B. Suresh ${ }^{1}$ \\ ${ }^{1}$ Infrared Imaging Center, Koneru Lakshmaiah Educational Foundation, Vaddeswaram, A.P, \\ India-522502. \\ E-mail.ID: gvs0raos@gmail.com
}

\begin{abstract}
In the recent past, Frequency modulated optical stimulus-based thermal wave imaging modality has gained much attention from past decades as a reliable non-destructive testing modality. Various processing techniques emerged in the frequency modulated thermal wave imaging (FMTWI) modalities for enhanced defect detection and depth resolution. The inadequate frequency resolution characteristics of Fourier transform for non-stationary signals recommend various processing techniques. The present article proposes a pulse compression based processing technique derived from the fractional Fourier transform to enhance the defect detection and depth resolution in quadratic FMTWI. An industrial composite material woth flat bottom holes is used for experimental verification of proposed methodology. Defect signal to noise ratio and size estimation concerned as figures of merits to validate the proposed method. A comparative analysis with conventional processing techniques such as Fourier transform-based phase analysis and pulse compression suggested that proposed time-frequency analysis presents enhanced defect detection.
\end{abstract}

Index Terms:Non-stationary thermal wave imaging, Fourier Transform, pulse compression, Fractional Fourier transform, Time-frequency analysis. 


\title{
Design of Ternary D-latch Using Graphene Nanoribbon Field Effect Transistor
}

\author{
BaduguDivya Madhuri ${ }^{1}$, S. Sunithamani ${ }^{1}$ \\ ${ }^{1}$ Department of ECE, Koneru Lakshmaiah Education Foundation, \\ Green Fields, Vaddeswaram, A.P., India-522502. \\ E-mail: nalluri.siddu@kluniversity.in.
}

\begin{abstract}
:
In this paper, the ternary D-latch design using Graphene Nanoribbon FETs (GNRFETs) is presented. The three valued logic (i.e., ternary logic) design is a best alternate solution to the existing binary logic as it provides smaller interconnects, faster computations and smaller chip area. Therefore, the design can allows the energy efficient, low-complex and high-speed circuits in modern digital design. The threshold voltage of the GNRFETs is varied by the graphene width, which depends on the number of dimer lines. The ternary D-latch proposed in our work is implemented utilizing the basic ternary logic designs such as negative and standard inverters and NAND gates. The simulations are carried out using HSPICE simulation tool. It has been observed that the ternary D-latch functioning is correctly implemented using the proposed circuit with GNRFETs
\end{abstract}

Index Terms-Graphene Nanoribbon FET, D-latch and HSPICE. 


\title{
Design and Implementation of Advanced 64-bit Pipelined Risc Processor for Several Applications
}

\author{
G.V.Ganesh ${ }^{1}$, N.Siddaiah ${ }^{1}$,M.S.G.Prasad ${ }^{1}$, P.Pardhasaradhi $^{1}$ \\ ${ }^{1}$ Department of ECE, Koneru Lakshmaiah Education Foundation, \\ Green Fields, Vaddeswaram, A.P., India-522502. \\ E-mail.ID: ganeshgorla_ece@kluniversity.in
}

\begin{abstract}
:
In this paper, we propose 64-bit pipelined RISC processor using VLIW architectures. Reduced instruction is the main criteria used to develop in this processor. With a single instruction scheme, more executions can be done using S.I.M.E. processor consists of the blocks namely program counter, clock control unit, ALU, IDU and registers. Advantageous architectural modifications have been made in the incrementor circuit used in program counter and carry select adder unit of the ALU in the RISC CPU core. In this paper we designed pipelined RISC processor using VLIW architecture to get better throughput and can be used to various applications.
\end{abstract}

Index Terms: RISC, Super scalar pipeline, VLIW, SIME 


\title{
Cryptography aes Algorithm Power Calculation using Clock Gating Technique
}

\author{
G.V. Ganesh ${ }^{1}$, N. Siddaiah ${ }^{1}$, M.S.G. Prasad ${ }^{2}$, P. Pardhasaradhi ${ }^{3}$ \\ ${ }^{123}$ Department of ECE, Koneru Lakshmaiah Education Foundation, \\ Green Fields, Vaddeswaram, A.P., India-522502. \\ E-mail.ID: ganeshgorla_ece@kluniversity.in
}

\begin{abstract}
:
Advanced Encryption Standard (AES). The AES is a Federal Information Processing Standard (FIPS). The AES algorithm is a symmetric block cipher that can encrypt, (encipher), and decrypt, (decipher), information. Encryption converts data to an unintelligible form called cipher-text. Decryption of the cipher-text converts the data back into its original form, which is called plaintext. The AES algorithm is capable of using cryptographic keys of 128, 192, and 256 bits to encrypt and decrypt data in blocks of 128 bits. Many algorithms were originally presented by researchers from twelve different nations. The Rijndael algorithm was chosen in this paper since it had the best overall scores in security, performance, efficiency, implementation ability and flexibility. In this paper low power clock gating technique is used to reduce power of AES core. Results show that dynamic power can be minimized as overall design is considered when compared to design without clock gating technique.
\end{abstract}

Index Terms: AES, clock-gating, Low-power, information security, Rijndael algorithm. 


\title{
Design and Fabrication of SAW Resonators on Silicon Substrate Employing Transverse BAW in Patterned AIN
}

\author{
Sai Krishna Santosh G' ${ }^{1}$, M. Lakshmana Kumar ${ }^{1}$ \\ ${ }^{1}$ Department of ECE, Koneru Lakshmaiah Education Foundation, \\ Green Fields, Vaddeswaram, A.P., India-522502. \\ E-mail: gsksantosh17@kluniversity.in
}

\begin{abstract}
:
The paper presents the design and fabrication of edge reflection type surface acoustic wave (SAW) resonators on silicon ( $\mathrm{Si}$ ) substrate employing transverse bulk acoustic wave (BAW) in periodically patterned AIN thin film. Two types of resonators are fabricated and tested: Patterned$\mathrm{AlN} / \mathrm{Si}$ and patterned-AlN/SiO$/ 2 / \mathrm{Si}$. Transverse $\mathrm{BAW}$ excited in the AlN pattern couples acoustically and transforms into surface wave in silicon substrate with a unique characteristic of high phase velocity and high coupling coefficient compared to conventional thin film SAW devices on silicon. The performance parameters of the proposed device are estimated through finite element simulations and SAW resonators are designed using equivalent circuit model and the parameters are compared with fabricated and tested devices.
\end{abstract}

Index Terms - resonator, hybrid transducer, SAW, BAW, thin film, patterned-AlN film 


\title{
Modelling and Simulation of Edge Reflection Type SAW Ladder Filters on Silicon Substrate using ZnO Films
}

\author{
Sai Krishna Santosh G' ${ }^{1}$, Lakshman Pappula ${ }^{1}$ \\ ${ }^{1}$ Department of ECE, Koneru Lakshmaiah Education Foundation, \\ Green Fields, Vaddeswaram, A.P., India-522502. \\ E-mail: gsksantosh17@kluniversity.in
}

\begin{abstract}
:
The paper presents the modeling and simulation of edge reflection type surface acoustic wave (SAW) ladder filters on silicon ( $\mathrm{Si}$ ) substrate using $\mathrm{ZnO}$ thin films. Series and parallel resonators are designed in order to match the series resonant frequency of parallel resonator with the antiresonant frequency of series resonators. Various ladder filters configurations are studied using finite element simulations to obtain the performance parameters like Q-factor, bandwidth, sidelobe rejection ratio, and insertion loss. FEM simulations are performed to obtain the performance parameters of ladder filters and results are compared with theoretical results.
\end{abstract}

Index Terms - resonator, hybrid transducer, SAW, ladder filters, thin film, patterned-AlN film 


\title{
IOT Based Global Navigation Satellite System (GNSS) Ground Monitoring System Using Cloud
}

\author{
Ali Baig Mohammad ${ }^{1}$, Durga Prasad Rajulapati \\ ${ }^{1}$ Assistant Professor, ECE Dept., Koneru Lakshmaiah Education Foundation, \\ Vaddeswaram, AP, India-522502 \\ E-mail.: mdabaig@kluniversity.in \\ ${ }^{2}$ Assistant Professor, ECE Dept., Ramachandra College of Engineering, \\ Eluru, Andhra Pradesh, India.
}

\begin{abstract}
:
These days, the Internet of Things is a quickly developing innovation that grants to include advanced gadgets into a system. Utilizing the Internet of things innovation to gather facts from an assortment of Internet associated GNSS beneficiaries gives an interesting chance to get continuous data about the uncommon and Fleeting movement of ionospheric attributes with excessive desires. The capacity to make a thick sensor arrangement is accomplished through the utilization of modest single-recurrence GNSS beneficiaries dependent on the Arduino innovation. This methodology can be actualized to acquire ongoing information on the all-out total electron content of the ionosphere. The assured information of the ionosphere deferral to radio signal of Global navigation for system satellite /Global positioning system satellite TV for PC and the estimation of the ionosphere TEC are accomplished straightforwardly within the Global navigation for system satellite beneficiary. The consequences are transmitted over a remote correspondence medium through Web to the cloud server, in which maps of the TEC to the ionosphere are developed. Now are proposed the new Architecture, by using this architecture we can directly store the data into the cloud. By using this architecture, we can provide remote monitoring.
\end{abstract}

Index Terms -IoT-(Internet of Things), TEC-(Total Electron Content), GNSS (Global Navigation Satellite System), GPS-(Graphical Positioning System). 


\title{
Velocity Synchronous Linear Chirplet Transform Based Subsurface Analysis in Non-Stationary Thermal Wave Imaging
}

\author{
G. V. P. Chandra Sekhar Yadav', V. S. Ghali ${ }^{1}$ \\ ${ }^{1}$ Infrared Imaging Center, Department of ECE, Koneru Lakshmaiah Education Foundation, \\ Green Fields, Vaddeswaram, A.P., India-522502. \\ E-mail: sekhar.yadav2008@gmail.com,gvs0raos@gmail.com.
}

\begin{abstract}
:
A thorough inspection and evaluation of materials is needed for several industrial applications such as aerospace and defense etc. Non Destructive Testing \& Evaluation (NDT\&E) techniques are used to test the integrity of the materials without diminishing its future usefulness. Out of several NDT\&E techniques active infrared thermography come into highest priority for testing and evaluation of materials, due to its intrinsic testing capabilities such as whole-field, non-contact and non-invasive abilities. Present work commence a novel post processing technique for recently proposed Quadratic Frequency Modulated Thermal Wave Imaging (QFMTWI) to explore the sub surface details of CFRP and GFRP test specimens. Defect detectability of the proposed technique is quantified by comparing signal to noise ratio, full width at half maxima with the conventional techniques.
\end{abstract}

Index Terms - Non-Destructive Testing \& Evaluation, Quadratic Frequency Modulated Thermal Wave Imaging, Signal to Noise Ratio. 


\title{
An Experimental Investigation of Correlation Based Matched Filter Approach Using Barker Coded Thermal Wave Imaging
}

\author{
G. V. P. Chandra Sekhar Yadav', V. S. Ghali ${ }^{1}$ \\ ${ }^{1}$ Infrared Imaging Center, Department of ECE, Koneru Lakshmaiah Education Foundation, \\ Green Fields, Vaddeswaram, A.P., India-522502. \\ E-mail: sekhar.yadav2008@gmail.com,gvs0raos@gmail.com.
}

\begin{abstract}
:
Active infrared thermography is becoming a prominent non-destructive testing modality to cater the demand for material characterization due to its intrinsic standards viz. whole field, fast, remote, qualitative and quantitative testing features. This work uses barker coded thermal wave imaging to test the integrity of composite material at different experimental time periods to facilitate fine subsurface details with improved resolution. Out of various post processing methods, the matched filter based correlation analysis is opted, since it increases the energy concentration in main lobe and provides considerably less energy in side lobes that leads to enhanced defect detection. This work experimentally studies the detection capabilities of barker coded thermal wave imaging at different experimental time periods for carbon reinforced polymer with artificial flat bottom holes. Further, matched filter based post processing technique is validated by considering the signal to noise ratio, full width at half maxima and peak to side level as figure of merits.
\end{abstract}

Index Terms - Barker coded thermal wave imaging, Matched filter, Peak to side level, Peak delay. 


\title{
Underwater Target Tracking using Fusion of Bearings
}

\author{
Koteswara Rao S ${ }^{1}$, Kausar Jahan ${ }^{1}$ \\ ${ }^{1}$ Department of ECE, Koneru Lakshmaiah Education Foundation, \\ Green Fields, Vaddeswaram, A.P., India-522502. \\ E-mail: rao.sk9@gmail.com, kausar465@ieee.org.
}

\begin{abstract}
:
Two sensor arrays, hull-mounted array, and towed array sensors are selected for bearings-only tracking. An algorithm is designed to integrate the information obtained as bearing measurements from both sensor arrays to provide a better solution. The use of data from two different sensor arrays minimizes the observability problem, and in order to achieve observability, the observer does not need to follow the S-maneuver. When measurements of the bearing are taken from a single sensor array, the performance of the fusion algorithm is equivalent to that of the algorithm. To assess the efficacy of algorithms more accurately, five different filters (Extended Kalman Filter. Modified gain bearings-only extended Kalman filter, Unscented Kalman filter, particle filter combined with extended Kalman filter and particle filter combined with Modified gain bearingsonly extended Kalman filter, Unscented Kalman filter) are used to assess both algorithms and Monte-Carlo loops. In terms of convergence times for solutions, the performance of the fusion algorithm is also calculated.
\end{abstract}

Index Terms - Bearings-only tracking, Data fusion, Hull mounted array, Towed array, Nonlinear filters. 


\title{
Passive Target Tracking Using Fusion of State Vectors from Two Sensor Arrays
}

\author{
Koteswara Rao $\mathbf{S}^{1}$, Kausar Jahan ${ }^{1}$ \\ ${ }^{1}$ Department of ECE, Koneru Lakshmaiah Education Foundation, \\ Green Fields, Vaddeswaram, A.P., India-522502. \\ E-mail: rao.sk9@gmail.com, kausar465@ieee.org.
}

\begin{abstract}
:
This research addresses the problem of improving state estimation of bearings-only underwater passive target tracking in real time. An algorithm is developed for multiple target detection and to fuse the state vectors if a single target is detected. For simplicity, two sensor arrays (Hull mounted array and towed array) are considered for tracking the targets. So, two targets with different and similar parameters of motion are considered for evaluation of the algorithm. To assess the efficacy of algorithm more accurately, five different filters (Extended Kalman Filter, Modified gain bearings-only extended Kalman filter, Unscented Kalman filter, particle filter combined with extended Kalman filter and particle filter combined with Modified gain bearings-only extended Kalman filter, Unscented Kalman filter) are used. Also, the results are compared with those obtained using single sensor. Monte-Carlo runs are done to evaluate the performance of algorithms more accurately using MATLAB software platform.
\end{abstract}

Index Terms - Bearings-only tracking, State vector fusion, Unscented Kalman filter, Data association. 


\title{
Detection of Dengue Disease by Using MEMS BIOSENSOR
}

\author{
Shameem Syed ${ }^{1}$, Chandan motamarri ${ }^{2}$, Charitha Alagadda ${ }^{3}$ \\ Department of ECE, Koneru Lakshmaiah Education Foundation, \\ Green Fields, Vaddeswaram, A.P., India-522502. \\ E-mail ID: shameemsyed@kluniversity.in
}

\begin{abstract}
:
Micro-Electro-Mechanical (MEMS) is a fast technology that carried different ideas in the fields like biomedical. MEMS got many applications in biomedical. Dengue is a break borne fever disease which is caused by dengue virus. This can be detected using MEMS bio-sensor. In this paper we use cantilever mechanism for the early stage detection of the disease. Here we use antibodies that are the bio-elements in the biosensor. The cantilever simulation result includes displacement and capacitance measurement which is the best method for dengue detection. Based on the amount of the displacement we can identify the severity of the disease.
\end{abstract}

Index Terms- biosensor, capacitance, displacement, antibody, and dengue. 


\title{
Capacitance Based Piezoelectric Energy Harvester
}

\author{
Shameem Syed ${ }^{1}$, Rama Krishna Prasad ${ }^{2}$, G Bhavya Lahari ${ }^{3}$, T Tarun ${ }^{4}$ \\ Department of ECE, Koneru Lakshmaiah Education Foundation, \\ Green Fields, Vaddeswaram, A.P., India-522502. \\ *E-mail ID: shameemsyed@kluniversity.in
}

\begin{abstract}
:
Now-a-days the utilization of energy in all the fields raised to saturation. But, the resources presently available are not sufficient for utilization. So, it is essential to use the available natural resources and convert them into the utilizable form called energy Harvesting. As, the MEMS are the reliable low power structure, here we are proposing a new methodology with piezoelectric based energy harvesters. The simulation was done with aluminum, PZT and silicon. For the three materials the power analysis and the sensitivity are calculated and simulated. Among the three PZT gives low power operation and high sensitive. The main advantage of the structure is the variation in the frequency caused by the intensity of the light radiation that incident on the PZT material. It is also observed that the sensitivity remains constant even though there will be a lot of variation in the frequency. The piezoelectric energy harvester cantilever arrayed beam produces power of 0.4496 Watts. The entire structure of cantilever is designed, analyzed and simulated using COMSOL MULTIPHYSICS 5.0 version.
\end{abstract}

Index Terms- PZT, sensitivity, energyharvester, cantilever. 


\title{
Design of AES based Cryptography System using Verilog HDL
}

\author{
Fazal Noorbasha ${ }^{1}$ \\ ${ }^{1}$ Department of ECE, Koneru Lakshmaiah Education Foundation, \\ Green Fields, Vaddeswaram, A.P., India-522502. \\ E-mail: fazalnoorbasha@kluniversity.in.
}

\begin{abstract}
:
The top secret word system is the most common process among justification techniques on the internet and is operated more simply and professionally than other methods. However, it is a at risk method against attacks. To beat over this trouble, AES based cryptography system has been used. So, in this paper we have created a module which is optimized and uses low-power cryptographic algorithm. Cryptography in the accessible world serves major function in data security. Cryptography means writing of secret codes (cipher text) which is in messy form and cannot be read except we have a just right key to decode it. The proposed method is AES algorithm (128 bit) followed by to generate an OTP. This FPGA system is design by using Verilog HDL.
\end{abstract}

Index Terms - AES, Cryptography, Verilog HDL, FPGA. 


\title{
Implementation of Optimized Radix 4 Booth Algorithm
}

\author{
Barma Venkata RamaLakshmi ${ }^{1}$, Fazal Noorbasha ${ }^{1}$ \\ ${ }^{1}$ Department of ECE, Koneru Lakshmaiah Education Foundation, \\ Green Fields, Vaddeswaram, A.P., India-522502. \\ E-mail: ramalakshmibarma468@gmail.com, fazalnoorbasha@kluniversity.in.
}

\begin{abstract}
:
The multipliers play a key role in performance of any system. But the major drawback is it consumes more power and area. To improve the performance and reduce the power consumption and area utilization there are many algorithms and techniques. In any multiplication algorithm the main intend is to reduce the partial product summation. One of the widely used and effective algorithms is booth algorithm. In this paper we have designed and implemented the radix 4 algorithm. In radix 4 algorithm the partial products are reduced to $n / 2$. The simulation results are carried out on Xilinx Vivado tool.
\end{abstract}

Index Terms - Booth multiplier, radix 4, encoding, partial product. 


\title{
FPGA Design of AES based Chiper and Decipher Cryptography System using Verilog HDL
}

\author{
Fazal Noorbasha ${ }^{1}$, Jitendra Hanuman Cheruvu ${ }^{1}$, Praveen Boina ${ }^{1}$, Sai Vamsi \\ Battineni $^{1}$ \\ ${ }^{1}$ Department of ECE, Koneru Lakshmaiah Education Foundation, \\ Green Fields, Vaddeswaram, A.P., India-522502. \\ E-mail: fazalnoorbasha@kluniversity.in.
}

\begin{abstract}
:
The secret word system is the most usual process among validation techniques on the internet and is operated more simply and efficiently than other methods. However, it is a susceptible method against attacks such as eavesdropping or replay attack. To overcome over this problem, AES based cryptography system is used. So, in this paper we created a module which is optimized and uses low-power cryptographic algorithm. Cryptography in the existing world serves significant function in data safety. Cryptography means writing of secret codes (cipher text) which is in jumbled form and cannot be read except we have a just right key to decipher it. The proposed method is AES algorithm (128 bit) followed by to generate an OTP. This FPGA system is design by using Verilog HDL.
\end{abstract}

Index Terms - FPGA, AES, Chiper, Decipher, Cryptography, Verilog HDL. 

Engineering and Management Research

A Peer Revieved Open Access International Journal

\title{
FPGA Implementation of Optimized Radix 4 and Radix 8 Booth Algorithm
}

\author{
Barma Venkata RamaLakshmi ${ }^{1}$, Fazal Noorbasha ${ }^{1}$ \\ ${ }^{1}$ Department of ECE, Koneru Lakshmaiah Education Foundation, \\ Green Fields, Vaddeswaram, A.P., India-522502. \\ E-mail: ramalakshmibarma468@gmail.com, fazalnoorbasha@kluniversity.in.
}

\begin{abstract}
:
The multipliers play a key role in performance of any system. But the major drawback is it consumes more power and area. To enhance the performance and decrease the power consumption and area consumption there are many algorithms and techniques. In any multiplication algorithm the main aim is to decrease the partial product summation. One of the widely used and effective algorithms is booth algorithm. In this paper we are designing and implementing the radix 4 and radix 8 booth algorithm. In radix 4 algorithm the partial products are reduced to $n / 2$, whereas in radix 8 algorithm we can reduce the partial products to $n / 3$ in the multiplier encoding. The simulation results are carried out on Xilinx Vivado tool.
\end{abstract}

Index Terms - Booth multiplier, Booth algorithm, radix 4, radix 8, encoding, partial product. 


\title{
Design and Analysis of Coax-Fed U-Slot Antenna for Wireless
}

\section{Applications}

\author{
M. Vasuja Devi ${ }^{1}$ \\ ${ }^{1}$ Department of ECE, Koneru Lakshmaiah Education Foundation, \\ Green Fields, Vaddeswaram, A.P., India-522502. \\ E-mail: vasujadevi@kluniversity.in.
}

\begin{abstract}
:
A multi frequency $\mathrm{U}$-slot patch antenna for wireless applications is presented. The proposed antenna operates at four frequencies of $1.4 \mathrm{GHz}, 5.3 \mathrm{GHz}, 6 \mathrm{GHz}$ and $6.9 \mathrm{GHz}$ with return loss of $19 \mathrm{~dB},-22.50 \mathrm{~dB},-21.05 \mathrm{~dB},-19.37 \mathrm{~dB}$ respectively. The antenna is designed with Rogers R04232 substrate with dielectric constant of 3.2 and dielectric loss 0.043 with dimensions of 47541.5 mm3. Coaxial feed technique is used as a feed to the antenna which is having characteristic impedance $50 \mathrm{ohm}$. The simulation studies were carried out using Ansys HFSS 15.0 Software.
\end{abstract}

Index Terms - Ansys, HFSS. 


\title{
Multiband Micro Strip Antenna Design for Space Research Applications
}

\author{
M. Vasuja Devi ${ }^{1}$ \\ ${ }^{1}$ Department of ECE, Koneru Lakshmaiah Education Foundation, \\ Green Fields, Vaddeswaram, A.P., India-522502. \\ E-mail: vasujadevi@kluniversity.in.
}

\begin{abstract}
:
Now a day's communication needs antennas with multiple band characteristics for to support and to use in special wireless applications. The designed antenna structure is constructed and simulated with the help of High Frequency Structure Simulator (HFSS) of 13.0 version. For this Micro strip antenna substrate material used is Rogers/duroid $5880(\mathrm{tm})$ with a dielectric constant of 2.2 and $1 \mathrm{~mm}$ of thickness. The antenna structure is an octagonal antenna with a feed width of $1 \mathrm{~mm}$ through the Micro strip feeding technique. The observed results are in ka-band, Q-band, u-band and v-band in that order and the return loss and VSWR also be observed and tabulated. The designed antenna is mostly preferred in application of fixed satellite services, Deep space research, terrestrial microwave communications radio astronomy studies, radar and other kind of researches.
\end{abstract}

Index Terms - VSWR, HFSS. 


\title{
Design of Nano scale Square Ring Resonator Band-Pass Filter Using Metal-Insulator-Metal
}

\author{
B. Surendra Kumar ${ }^{1}$, M. Sridhar ${ }^{1}$ \\ ${ }^{1}$ Department of ECE, Koneru Lakshmaiah Education Foundation, \\ Green Fields, Vaddeswaram, A.P., India-522502. \\ E-mail: sridhar.m@kluniversity.in.
}

\begin{abstract}
:
In this paper, metal-insulator-metal (MIM)-based single-band plasmonic band-pass filters (BPF) design and analysis is presented. The basic design comprises a square ring resonator (SRR) which is coupled using straight waveguide with gap. The straight waveguide SRR is responsible for a single-band-operating wavelength (1355 nm, i.e., $221.24 \mathrm{THz})$. Design and simulations are carried out using finite-difference time-domain (FDTD)-based solver embedded in the Computer Simulation Technology (CST) Microwave Studio suite. The proposed filters are used for plasmonic single-band band-pass filter (BPF) applications in photonic integrated circuits (PICs).
\end{abstract}

Index Terms - MIM, photonic integrated circuits, SRR, BPF. 


\title{
Performance Analysis of Micro-Cantilever Shape Bio-Sensor for Swine Flu Detection
}

\author{
E. Anil ${ }^{1}$, S. Sunithamani ${ }^{2}$, K.B.Mehar Chand ${ }^{3}$, M. Surya Teja ${ }^{4}$, B. Pavani ${ }^{5}$ \\ ${ }^{2}$ Associate professor,Department of Electronics and Communication Engineering \\ Koneru Lakshmaiah Education Foundation, \\ Vaddeswaram, Guntur, Andhra Pradesh, India-522502. \\ E-mail: sunithamani.s@kluniversity.in
}

\begin{abstract}
:
Micro cantilevers are main component in any MEMS based biosensor systems.Micro electro mechanical Systems (MEMS) is the innovation of the little gadgets, they are partitioned and unmistakable from the speculative vision of sub-atomic nanotechnology and hardware. MEMS aremicroscopic device normally fabricated on silicon wafers. MEMS are comprised of segments near 1 and 100 micrometers in estimate (i.e., 0.001 to $0.1 \mathrm{~mm}$ ), and MEMS gadgets for the most part run in measure from 20 micrometers to a millimeter. They as a rule comprise of a focal unit that procedures information (the microchip) and a few segments that cooperate with the surroundings, for example, micro sensors. The stretch is transduced towards the site at which particles of the functionalized layer are associated with the cantilever surface. Micro sensors converts the deliberate mechanical energy in to an electrical energy. Generally, the materials such as Silicon, Polymers, are used in MEMS manufacturing process. These materials are used to get the required shape of devices and sensors used for fabrication. This paper describes the analysis of effect of micro cantilever shape in swine flu detection.
\end{abstract}

Index Terms - Biosensor, mems, cantilever shape. 


\title{
A Robust IoT Based Global Navigation Satellite System (GNSS) Ground Monitoring System Using Cloud
}

\author{
Ali Baig Mohammad ${ }^{1}$, Durga Prasad Rajulapati ${ }^{2}$ \\ ${ }^{1}$ Assistant Professor, Department of ECE, Koneru Lakshmaiah Education Foundation, \\ Vaddeswaram, A.P., India-522502. \\ E-mail: mdabaig@kluniversity.in \\ ${ }^{2}$ Assistant Professor, ECE Dept., Ramachandra College of Engineering, Eluru, Andhra \\ Pradesh, India.
}

\begin{abstract}
:
These days, the Internet of Things is a quickly developing innovation that grants to include advanced gadgets into a system. Utilizing the Internet of things innovation to gather facts from an assortment of Internet associated GNSS beneficiaries gives an interesting chance to get continuous data about the uncommon and Fleeting movement of ionospheric attributes with excessive desires. The capacity to make a thick sensor arrangement is accomplished through the utilization of modest single-recurrence GNSS beneficiaries dependent on the Arduino innovation. This methodology can be actualized to acquire ongoing information on the all-out total electron content of the ionosphere. The assured information of the ionosphere deferral to radio signal of Global navigation for system satellite /Global positioning system satellite TV for PC and the estimation of the ionosphere TEC are accomplished straightforwardly within the Global navigation for system satellite beneficiary. The consequences are transmitted over a remote correspondence medium through Web to the cloud server, in which maps of the TEC to the ionosphere are developed. Now we have proposed the new Architecture, by using this architecture we can directly store the data into the cloud. By using this architecture, we can provide remote monitoring.
\end{abstract}

Index Terms - IoT-(Internet of Things), TEC-(Total Electron Content), GNSS (Global Navigation Satellite System), GPS-(Graphical Positioning System). 


\title{
Modelling and Prediction of Covid-19 For Tamil Nadu, India
}

\section{Chelladurai $\mathbf{R}^{1}$, Sri Phani Kishore $\mathbf{D}^{2}$, Lingeshwaran $\mathbf{N}^{3}$, Ramesh Kumar $\mathbf{R}^{4}$}

\author{
1,2,3,4 Department of ECE, Koneru Lakshmaiah Education Foundation, \\ Green Fields, Vaddeswaram, A.P., India-522502. \\ E-mail: chelladurair@kluniversity.in
}

\begin{abstract}
:
A new type of Corona virus called COVID-19 has started to rapidly spread across the world. The impact and the death rate due to COVID-19 is very high when compared to other kind of Corona virus, and also, the spreading rate is also very high due to many asymptotic cases. Therefore, it is necessary for the nations to predict the cumulative confirmed cases and the average rate of the spreading in order to take necessary precautionary steps to avoid huge mortality rate. Based on the study conducted on the data obtained from WHO for different countries like Italy, Spain, France, Germany, Japan and South Korea between the dates 15th February and 10th May, we have predicted the cumulative confirmed cases, Average rate of spreading and the actual affected persons for Tamil Nadu based on the moving average model applied to the auto Correlation coefficient and the average rate of spreading till June 4th, 2020.
\end{abstract}

Index Terms - DWT, PCA, ICA, LBP, HOG, GLCM, HARRIS CORNER. 


\title{
A System of Beholding Patient and Hospital Management Using RFID
}

\author{
Chelladurai R $^{1}$, Sumanth Kumar Motamarri ${ }^{2}$, Suman Pullamsetti ${ }^{3}$, Sai \\ Krishna Sanagapalli ${ }^{4}$, Purnitha Desineedhi5 \\ ${ }^{1}$ Department of ECE, Koneru Lakshmaiah Education Foundation, \\ Vaddeswaram, A.P., India-522502. \\ E-mail: chelladurair@kluniversity.in
}

\begin{abstract}
:
Health is one of the vital aspects that should be taken into consideration in these days. Every human-being in the world should possess good health, if not they are in danger. As the technology is increasing day by day, we are experiencing new methodologies and technologies especially in the field of medicine. In these days, monitoring and management of patient health is becoming difficult. Suppose if a patient has undergoing several treatments, maintaining all those records and remembering and handle those each and every piece of data is much difficult. As the Internet of Things is developing very rapidly in the $21^{\text {st }}$ century, here we are proposing a model for the management of patient data using Internet of Things. With the help of Internet of Things (IoT), we can store our data and access at hospital. For this purpose, we are designing a prototype with the help of RFID technology interfacing with nodeMCU controller, earlier the management of records has been done in the form of papers, and computers.
\end{abstract}

Index Terms - DWT, PCA, ICA, LBP, HOG, GLCM, HARRIS CORNER. 


\title{
An Optimal Hybrid Solution to Local and Global Facial Recognition Through Machine Learning
}

\author{
K. Raju ${ }^{1}$, B.Chinna rao $^{2}$, N.L.Pratap ${ }^{3}$ \\ ${ }^{1}$ Department of ECE, Narasaraopeta Engineering College, Narasaraopet, Guntur, AP, India. \\ raju.kolluri@gmail.com \\ ${ }^{2}$ Department of ECE, NS Raju Institute of Technology, Visakhapatnam, India. \\ profbcrao@gmail.com \\ ${ }^{3}$ Nalajala Lakshman Pratap, Asst Prof, Department of Electronics and Communication \\ Engineering, Koneru Lakshmaiah Education Foundation, Vaddeswaram, AP, India. \\ E-mail: lakshmanpratap@kluniversity.in
}

\begin{abstract}
:
Face recognition need is fine assured as enormous industrial relevance use them to implement one or another objective.As facial recognition has beating advantages over other industrial applications and human eyes can easily evaluate performance, improved algorithms and smaller computing costs are continuously improving this methodology. The research starts with basic computation of global face features using Principal Component Analysis (PCA), Discrete Wavelet Transform (DWT) and Independent Component Analysis (ICA), with some standard classifiers like Neural Network (NN) and Support Vector Machine (SVM). It has observed that as learning rate are high in machine learning then accuracy of system goes high but increases the area and cost overhead. To overcome that training limitation, a Fusion based methods has been proposed in further work, based on Harris corner, Speed Up Robust Features (SURF) and DWT+PCA system model where only $10 \%$ training sample has been taken on Essex database and $99.45 \%$ accuracy is achieved. But to create the Fusion rule it requires some hit and trail methods which may not be Universal in every database. To overcome this limitation further an efficient Hybrid method proposed which elaborates the local features Linear Binary Pattern (LBP), Histogram Oriented Gradients (HOG), Gabor wavelet and global features (DWT, PCA) of face. Further these features trained with Neural Network classifier to obtained better accuracy nearly $99.40 \%$ with single image training from each class.
\end{abstract}

Index Terms - DWT, PCA, ICA, LBP, HOG, GLCM, HARRIS CORNER. 


\title{
Data Security in Cloud Computing Using Three-Factor Authentication
}

\author{
Sunanda Nalajala ${ }^{1}$, B. Moukthika ${ }^{1}$, M. Kaivalya ${ }^{1}$,N.L.Pratap ${ }^{2}$ \\ ${ }^{1}$ Department of CSE, Koneru Lakshmaiah Education Foundation, \\ Green Fields, Vaddeswaram, A.P., India-522502. \\ E-mail: sunanda@kluniversity.in. \\ ${ }^{2}$ Department of Electronics and Communication Engineering, Koneru Lakshmaiah Education \\ Foundation, Green Fields, Vaddeswaram, A.P., India-522502 \\ E-mail: lakshmanpratap@kluniversity.in
}

\begin{abstract}
:
Cloud storage is tremendously increasing its services, huge range of storage and communication of massive data over network. This practically has an adverse effect on the way of upholding this data, when it especially comes to the privacy of the user-secured and highly confidential data. We first view you with a system that is vulnerable to this authentication protocol with its misuse of biometrics and incorrect password generates no user to lost the mobile device. We even went along with this scheme and gave out a major issue to overcome with impersonation attack. However, this scheme makes the way easy to attack for offline password guessing attack. We included a three-factor authentication which carries the smart card into card reader, gets the password and identity and conveys the user details' requesting time. We then came up with a scheme to overcome these security flaws of this prescribed authentication scheme combining passwords, mobile devices and biometrics. The proposed system is robust three-factor authentication with the help of password, biometrics and mobile device which provides reliable security strength to the user's data and makes counterattack to existing attack, giving with more benefits compared to the previous scheme. This scheme will not only encounters with the security issues, but also provides with most enhanced security functionalities.
\end{abstract}

\section{Index Terms -Three-factor authentication Counter attacks Privacy Biometrics Smart cards}




\title{
International Journal for Innovative
} Engineering and Management Research

A Peer Revieved Open Access International Journal

\section{Vigorous Image Transmission Using Turbo Codes}

\author{
${ }^{1} \mathrm{M}$ Venkateswara rao \\ ${ }^{1}$ Department of ECE, Koneru Lakshmaiah Education Foundation, \\ Green Fields, Vaddeswaram, A.P., India-522502. \\ E-mail: venkateswararao@kluniversity.in
}

\begin{abstract}
:
The pictures that are sent through loud channels are amazingly to bit mistakes, which can seriously degenerate the nature of the pictures at the recipient. To send the compacted pictures through loud channels with low SNR, another of turbo codes, Bit plane cutting and 2-D separating is presented in this paper. In the spot plane cut, the mechanized $\mathrm{N}$-digit picture is apportioned into $2 \mathrm{~N}$ quantization levels. All of the $\mathrm{N}$ pieces is encoded by a convolution-based Turbo encoder and send through the Additive White Gaussian Noise channel. At the collector end, each piece plane is prepared through Turbo decoder and reassembled to shape the reproduced picture. This reproduced picture went through the 2-D channels to improve Peak to Signal clamor Ratio(PSNR).This framework has extra-conventional outcomes at low SNR esteems and give better PSNR values even at - $1 \mathrm{db}$, contrasted and conventional Turbo coding plan.
\end{abstract}

Index Terms -Bit plane slicing, Convolutional based Turbo encoder, Turbo decoder, PSNR, 2-D filter. 


\title{
Authentication Process in Smart Card Using Sha-256
}

\author{
V. Teju ${ }^{1}$ \\ ${ }^{1}$ Department of ECE, Koneru Lakshmaiah Education Foundation, \\ Green Fields, Vaddeswaram, A.P., India-522502. \\ E-mail: teju@kluniversity.in.
}

\begin{abstract}
:
Smart cards are related to the issues of security. The applications of smart card are manipulated in hardware, software and telecommunications which appears to be a big issue. In this paper we introduce a new design which is a secured 2 level processing in authentication which uses the number of the pin as 1st authenticated level using SHA-256. OTP is brought as a 2nd authenticated processing level. The project is to generate an OTP using the sha-256 hashing algorithm. As the program initiates, 'local host: 8080/view' is typed in the web browser to bring up the front end of the application. Client and server nodes are created in the client node, the column for the username, credentials, messages and the trust values created while on server node, name and user credentials are created.
\end{abstract}

Index Terms - Smartcards2, Security, Authentication, OTPs, SHA-256. 


\title{
Mining Environment Monitoring Based on Laser Communication with Internet of Things
}

\author{
V. Teju \\ Department of ECE, Koneru Lakshmaiah Education Foundation, \\ Green Fields, Vaddeswaram, A.P., India-522502. \\ E-mail: teju@kluniversity.in.
}

\begin{abstract}
:
Environment conditions like humidity, temperature and gas concentrations are checked to safeguard the people in mines. The equipment comprised of hardware like a microcontroller. This standard intends to decrease the risk of injury and illnesses, these accidents can lead misfortunes because of the mine safety. To protect the excavators from ecological perilous, we need to speak with diggers in the underground segment the information should initially be estimated. This measured data must be saved for future reference. The transmission is done using laser communication. The parameters measured in this project are: (i) temperature, (ii) humidity and (iii) gas concentration. The obtained sensor values are sent through laser transmission and the sent values are passed through the Wi-Fi router and the mining data is stored in the database.
\end{abstract}

Index Terms - Hazardous, Monitored, Segment, Premiums, Retirements 


\title{
Investigation on Cluster based Application Mapping Approach for Power Optimization in NoC
}

\author{
Aravindhan \\ ${ }^{1}$ Department of ECE, Koneru Lakshmaiah Education Foundation, \\ Green Fields, Vaddeswaram, A.P., India-522502. \\ E-mail: aravindhan@kluniversity.in.
}

\begin{abstract}
:
Network-on-Chips (NoCs) are one of the emerging research area that has several research problems that need to be addressed. Reducing the power consumption itself is one important problem for NoC based systems. Various methods are proposed till now to reduce the power consumption in NoC. Unless, for getting better results, cluster based mapping method is proposed. In this paper we propose a new cluster based mapping method is proposed with FM algorithm for reducing power consumption. On the other hand, an optimization technique called Tabu search is used for reducing power consumption. The performance and efficiency of proposed methodology was verified with the experiments conducted in different benchmarks in NoC. Experimental results shows that Tabu search is unsuitable for power calculation.
\end{abstract}

Index Terms - DWT, PCA, ICA, LBP, HOG, GLCM, HARRIS CORNER. 


\title{
International Journal for Innovative
} engineering and Management Research

A Peer Revieved Open Access International Journal

\section{Modified Bat Algorithm for Cluster based Application Mapping Method in 2D NoC}

\author{
Aravindhan \\ ${ }^{1}$ Department of ECE, Koneru Lakshmaiah Education Foundation, \\ Green Fields, Vaddeswaram, A.P., India-522502. \\ E-mail: aravindhan@kluniversity.in.
}

\begin{abstract}
:
Due to the continuous scaling in the design of large-scale integrated circuits (VLSI), the networkon-chip (NoC) has become one of the growing research areas and has to solve many research problems.In this study, we propose a modified BAT algorithm (MBA) for successful cluster-based bandwidth and power awareness mapping system for $2 \mathrm{D} \mathrm{NoC}$. The proposed method is verified by the number of experiments conducted in the NoC with different types of standard definitions.
\end{abstract}

Index Terms - DWT, PCA, ICA, LBP, HOG, GLCM, HARRIS CORNER. 


\title{
International Journal for Innovative Engineering and Management Research
}

A Peer Revieved Open Access International Journal

\section{Design and Simulation of All-Optical XOR Gate and its implementation in Binary to Gray Code \& Gray to Binary Code Converter using Semiconductor Optical Amplifier}

\author{
Vipul Agarwal \\ ${ }^{1}$ Department of ECE, Koneru Lakshmaiah Education Foundation, \\ Green Fields, Vaddeswaram, A.P., India-522502. \\ E-mail: viulagarwal1@kluniversity.in.
}

\begin{abstract}
:
The present paper also explores the techniques that are used to reduce attenuation, improve power and to increase speed in optical communication. Gray codes are widely used to facilitate error correction in digital communications. An all-optical four-bit binary to Gray code and Gray to binary code converter with the help of semiconductor optical amplifier is proposed and described here. However, here it has been used to design optical gray to binary code converter by two techniques. Initially by using travelling wave SOA and second by using MZI (Mach- Zehnder Interferometer).These two techniques are compared in terms of extinction ratio and outputs. On the basis of this criteria, all circuits are implemented successfully at $10 \mathrm{~GB} / \mathrm{s}$ with the means of OptiSystem software. The advanced signal processing in next generation optical networks demands all optical digital devices as the key element which provides the benefit in terms of power, speed and cost. In this paper, execution of optical binary to gray \& Gray to Binary code converter with the means of XOR gate is presented. The mechanism of co-propagating MZI configuration which holds SOA symmetrically in both arms is opted to design XOR gate. The extinction ratio obtained is $44.59 \mathrm{~dB}$ in XOR gate and $45.307 \mathrm{~dB}$ in binary to gray code converter at $10 \mathrm{~GB} / \mathrm{s}$.
\end{abstract}

Index Terms - Hazardous, Monitored, Segment, Premiums, Retirements 


\title{
Ultrafast All Optical Parity Generator and Checker Based on Quantum dot Semiconductor Optical Amplifier
}

\author{
Vipul Agarwal \\ ${ }^{1}$ Department of ECE, Koneru Lakshmaiah Education Foundation, \\ Green Fields, Vaddeswaram, A.P., India-522502. \\ E-mail: viulagarwal1 @ kluniversity.in.
}

\begin{abstract}
:
This investigation reports all optical parity generator and checker based on quantum dot semiconductor optical amplifiers at $200 \mathrm{~Gb} / \mathrm{s}$. The proposed circuit has been realized by using SOA-MZI configuration with quantum dot as active region in SOA. Parity generator consists of three inputs and utilizes 2 XOR logic while parity checker employs 3 XOR logic with 4 inputs. Cross phase modulation in SOA has been exploited in the design of XOR logic. MZI configuration converts phase modulation to amplitude modulation to achieve desired Boolean equation of XOR. Wide and clear eye diagram has been obtained with extinction ratio above $10 \mathrm{~dB}$ and $\mathrm{Q}$ factor of above 12 . Obtained results validates feasibility and confirms practical implementation of proposed scheme.
\end{abstract}

Index Terms - Hazardous, Monitored, Segment, Premiums, Retirements 


\title{
Design and Implementation of High Entropy TRNG using FPGA
}

\section{Ch Priyanka}

\author{
${ }^{1}$ Department of ECE, Koneru Lakshmaiah Education Foundation, \\ Green Fields, Vaddeswaram, A.P., India-522502. \\ E-mail: priyankach@kluniversity.in
}

\begin{abstract}
:
In this paper, a novel ring based double entropy core TRNG engineering on FPGA with high working recurrence and high throughput has been performed and introduced. The plan of double entropy core TRNG has been created by joining together the confused framework based RNG and the RO-put together RNG structures with respect to FPGA. The clamorous oscillator structure as the fundamental entropy source has been actualized in VHDL utilizing Euler mathematical calculation in 32-cycle IQ-Math fixed point number standard on FPGA. The designed oscillator has been integrated for the FPGA chip and the insights identified with chip asset utilization and clock frequencies of the units have been introduced. The RO-based RNG structure has been planned as the subsequent entropy source. Chaos ring based double entropy core novel TRNG unit have been made by joining of these two FPGA-based structures in the XOR work utilized at the post preparing unit. The throughput of the planned double entropy core TRNG unit ranges 464 Mbps.
\end{abstract}

Index Terms - FPGA, Verilog, CORDIC 


\title{
Hand-Drawn Circuit Recognition with Deep Learning
}

\section{Musala Venkateswara Rao}

\author{
Department of ECE, Koneru Lakshmaiah Education Foundation, \\ Green Fields, Vaddeswaram, A.P., India-522502. \\ E-mail: venkatvlsi@kluniversity.in.
}

\begin{abstract}
:
In electrical science, a circuit outline is a graphical depiction of an electrical circuit that contains fundamental picture to address the electrical fragments and accessibility between parts. For example, a resistor in an electrical diagram has a value and two relationship with various parts. People can see these electrical parts using their understanding which they recently arranged. Starting there, it is relied upon to truly enter the portions into machine to play out the related strategy even the graph is puzzled with massive amounts of fragments. This procedure is not viable if there ought to emerge an event of unusualness of the graphs. In this paper we proposed another circuit confirmation technique in which the machine can coordinate examine a bewildering circuit outline. The Combination of various component symbols and their connections gives an Electrical Circuit. Here we present an image segmentation technique which perceives the hand-drawn circuit diagrams. The picture which is drawn by hand will be examined and that filtered picture of the hand-drawn circuit chart will be pre-prepared so we can expel the un-needed pieces of the graph and convert that into bilevel. An electrical circuit is generally made from portion pictures and their affiliations. This endeavor proposes a structure for modified affirmation of hand-drawn electrical circuits subject to picture division and game plan figuring's. Each part picture is expelled from affiliation wires, by then a distinctive verification method is applied to each part of the image. The yield of system is a netlist that perceives the picture types and interconnections between them. The netlist can be used to make PC schematic of circuit.
\end{abstract}

Index Terms - Graphical Depiction, Image Segmentation, Scanned Image, Electrical Circuit, Netlist, PC Schematic. 


\title{
FPGA Implementation of Efficient PCA Technique
}

\section{Ch Priyanka}

\author{
${ }^{1}$ Department of ECE, Koneru Lakshmaiah Education Foundation, \\ Green Fields, Vaddeswaram, A.P., India-522502. \\ E-mail: priyankach@kluniversity.in
}

\begin{abstract}
:
The aim of the proposal is to build up a productive equipment usage of the PCA (Head Component Analysis) calculation for dimensionality decrease in hyperspectral imaging. PCA incorporates the computation stages as standardization, covariance lattice count and eigenvalue decay. A productive equipment execution of covariance network count has been planned in the specialization venture which utilizes high parallelism. The principle center is performing eigenpair disintegration around the covariance lattice and utilizing BRAM (block RAM) as middle stockpiling with the end goal that an enormous covariance network can be disintegrated in a reduced module. Completely defined FPGA usage of eigen deterioration is created and performed on the covariance framework. Cyclic-by-column Jacobi strategy is utilized to discover all the eigenvalues and eigenvectors simultaneously. CORDIC calculation is utilized with Jacobi for more effective execution. The Jacobi is planned so that it can adjust to any FPGA paying little mind to the measure of accessible assets. This permits discovering answers for tremendous dimensional grids in even little measured FPGAs, e.g $100 \times 100$ by relinquishing a limited quantity of execution speed. The entire synthesizable PCA configuration is demonstrated utilizing VHDL endeavoring to streamline the plan. Zedboard Zynq-7000 arrangement is utilized as the FPGA. The plan is tried on MATLAB. All equipment modules are blended utilizing Xilinx Vivado-2017.3 device.
\end{abstract}

\section{Index Terms - FPGA, Verilog, CORDIC}




\title{
Detection of Objects for Autonomous Cars Using Lane Detection Method
}

\author{
P Poorna Priya \\ Department of ECE, Koneru Lakshmaiah Education Foundation, \\ Green Fields, Vaddeswaram, A.P., India-522502. \\ E-mail: poornariya@kluniversity.in.
}

\begin{abstract}
:
This paper presents two algorithms, one to detect, track objects and the other for lane line detection. This detection and tracking of objects are done by using a Tensor flow object detection API. And a simple computer vision technique is obtained for Lane line detection. The objects Location which are identified that is forwarded to the algorithm of object detection and tracking. The object detection tracking system algorithm is used for obtain by using CVV. The proposed approach can able to detect objects in different illumination and occlusion. It accuracy of achieved is $90.88 \%$ on self-generated image sequences
\end{abstract}

Index Terms - Object, system, CVV, Tracking. 


\title{
Design of Birds Detector and Repellent Using Frequency Based Repeller by Machine Learning Algorithm
}

\author{
P Poorna Priya \\ Department of ECE, Koneru Lakshmaiah Education Foundation, \\ Green Fields, Vaddeswaram, A.P., India-522502. \\ E-mail: poornariya@kluniversity.in.
}

\begin{abstract}
:
Among entire globe many Countries people work as farmers to survive their lives . Plants are interconnected to farming and pests which are related to plants, where there are bulk of plants then they lead to farming there must be effect causing animals called pests. Pests are not only animals they may be birds too that damage the plants. One of the example of pests are birds and monkeys that eat plants and its products and cause harm to farmers. In This research technique we has developed and designed a prototype of birds detector and repellent of birds based on frequency. This designed model has a Bird detection and repellent system which detects the bird based on its given python code as well as with the help of some machine learning algorithms and then proceeds further step to a repellent which produces a certain frequency with respective the birds by classification the birds based on there resonating frequencies, so the bird will be disturbed through the repellent frequency and goes away fron it. This designed model consists of python open cv software as a detector to detect movement from birds and further classifies the type of bird and for that type it give signals to further step to fire certain signal frequency to repeller.
\end{abstract}

\section{Index Terms - Repeller, bird, frequency, time.}




\title{
Transition Analysis of Beam Scintillation in R-FSOC Links Using Discrete Markov Chain Model
}

\author{
Rahul Bose \\ Department of ECE, Koneru Lakshmaiah Education Foundation, \\ Green Fields, Vaddeswaram, A.P., India-522502. \\ E-mail: rahulbosu@kluniversity.in.
}

\begin{abstract}
:
The intent of this chapter is to estimate the turbulence inflicted instantaneous beam scintillation level in an optical reflector-assisted FSO link by incorporating a CSI estimation technique that does not require the knowledge of the power of the optical beam at the Tx. The concept of the propounded CSI determination technique is then illustrated by estimating the optical scintillation level in an OOK based R-FSOC test bed. A FSMC model is then developed for analyzing the unconditional and stationary transitions in the laser scintillation level in the R-FSOC test bed. Discrete turbulence states are first perceived in the non-uniform temperature induced turbulent regimes. Thereafter, the BER performance of the CSI based R-FSOC system is analyzed to demarcate the SNR admissible region for each turbulence state. By comparing the instantaneous SNR of the received signal with the SNR admissible region, the transition probability of the scintillation process to all the possible transition states are evaluated, and the O-TSTP matrix is developed for the artificially fostered turbulent channel. By incorporating the Eigen value and limiting probability analysis on the O-TSTP matrix, the FSMC mixing time and the scintillation rates for the experimental link are estimated to be $3.71 \mathrm{~s}$ and $4.09 \%$ respectively.
\end{abstract}

Index Terms - SNR, BER, CSI, Repeller, bird, frequency, time. 


\title{
Analytical Tracking of Beam Fluctuations in Random Media
}

\section{Rahul Bose}

\author{
Department of ECE, Koneru Lakshmaiah Education Foundation, \\ Green Fields, Vaddeswaram, A.P., India-522502. \\ E-mail: rahulbosu@kluniversity.in
}

\begin{abstract}
:
This paper demonstrates the implementation of specific perturbation techniques to analytically track the distortions in the optical beam propagation in the linear and non-linear media with fluctuating atmospheric refractive indices. The order of the ODE that characterizes the optical field propagation is not affected by the perturbed RI. The asymptotic solutions generated through RP method comprises of secular terms that result in tracking error in proportion to the beam propagation distance. The negative effects of the secular terms are significantly reduced by: (a) obtain CFs with HOTs, or (b) employing PL perturbation technique. The asymptotic solutions generated through RP and PL perturbation methods are observed to trail the optical fluctuations with minimal tracking error. The RP technique is incorporated in for deriving the instantaneous CSI in order to gauge the turbulence induced scintillation level across a reflector assisted FSOC link without the knowledge of the transmitted beam power.
\end{abstract}

Index Terms -HOT, FSOC, Beam, perturbation. 


\title{
Design of Nanoscale Square Ring Resonator BPF Using MIM Waveguide
}

\author{
${ }^{1}$ Surendra Kumar Bitra, ${ }^{2}$ Sridhar M \\ ${ }^{1}$ Research scholar, Dept of ECE, KLEF, \\ Vaddeswaram, Andhra Pradesh, India. \\ ${ }^{2}$ Professor, Dept of ECE, Vaddeswaram, Andhra Pradesh, India. \\ E-mail: bitrasurendrakumar@gmail.com, \\ sridhar.m@kluniversity.in
}

\begin{abstract}
:
In this Work, Metal-Insulator-Metal (MIM) based single band plasmonic bandpass filters (BPF) design and analysis is presented. The basic design comprises of a Square Ring Resonators (SRR) is coupled using straight wave-guide with gap. The straight waveguide SRR is responsible for a single band operating wavelength (1355 nm, i.e. $221.24 \mathrm{THz})$. Design and simulations are carried out using Finite Difference Time Domain (FDTD) based solver embedded in the Computer Simulation Technology (CST) Microwave Studio suite. The pro-posed filters are used for plasmonic single band bandpass filter (BPF) applications in photonic integrated circuits (PIC's).
\end{abstract}

Keywords: MIM, Photonic Integrated Circuits, SRR and BPF. 


\title{
ARMA Model for Short Term TEC Prediction
}

\author{
D. Venkata Ratnam \\ Department of ECE, Koneru Lakshmaiah Education Foundation, \\ Green Fields, Vaddeswaram, A.P., India-522502. \\ E-mail: dvratnam@kluniversity.in.
}

\begin{abstract}
:
The applications of Global Positioning System (GPS) are increasing enormously day by day and hence the need for accurate positioning using GPS. The accuracy of GPS is affected by various factors in atmosphere, but the delay in the ionosphere is the most significant. The ionospheric delay is proportional to the total electron content (TEC) of the ionosphere. The modeling of the TEC plays an important role in the precise positioning using GNSS systems. A time series model based on Auto Regressive Moving Average (ARMA) to perform short-term ionospheric TEC predictions is developed for predicting the regional ionospheric TEC using GPS data acquired at KL University, Guntur, India. The outcome of this work would be immensely useful understanding space weather and navigation applications.
\end{abstract}

Index Terms - GPS, TEC, ARMA, prediction, space weather. 


\title{
Forecasting of GPS Ionospheric Time Delays Using Fuzzy Inference Systems
}

\author{
D. Venkata Ratnam \\ Department of ECE, Koneru Lakshmaiah Education Foundation, \\ Green Fields, Vaddeswaram, A.P., India-522502. \\ E-mail: dvratnam@kluniversity.in.
}

\begin{abstract}
:
Fuzzy Logic (FL) is a particular area of interest in the study of Artificial intelligence (AI) based on the idea that in fuzzy sets each element in the set can assume a value from 0 to 1 , not just 0 or 1 , as in classic or crisp set theory. Fuzzy inference system is composed of set of membership functions and knowledge base (fuzzy rules).The degree of membership is a measure of the element's belonging to the set. GPS positional accuracy is limited by several error sources such as ionosphere, troposphere, pseudorange measurement noise etc. Among all, ionospheric time delay error is predominant error. The ionospheric state is largely determined by solar forcing. Hence, there is a need to develop ionosphere forecasting model. In this paper, Fuzzy Logic technique is implemented using GPS receiver data. The ionospheric time delay is a function of Total Electron Content (TEC) these values are measured using GPS data. Data from GPS station located at $\mathrm{K} \mathrm{L}$ University $\left(16.44^{\circ} \mathrm{N}, 80.62^{\circ} \mathrm{E}\right)$ Guntur is considered for the analysis. The TEC data from March 1-10, 2013 is considered for the analysis. The focus is on the efficiency of the proposed optimization method for TEC prediction. Gradient descent optimization technique is investigated for improving the prediction accuracy. The fuzzy modeling of the sequence similarity has been undertaken as design of the Suzeno fuzzy model with four inputs, with each 3 trapezoidal membership function using with grid partition. It is found that ANFIS with LDIVIDE method is not able to follow the actual data. It is evident that the predicted TEC values are closely following the true TEC measurements for Gradient Descent method. More number of stations data over several seasons would be useful for forecasting ionospheric characteristics using the proposed technique.
\end{abstract}

Index Terms - Fuzzy logic, TEC, GPS, ionospheric time delay, gradient descent. 


\title{
International Journal for Innovative Engineering and Management Research
}

A Peer Revieved Open Access International Journal

\section{Analysis of 6Transistor Full Adder}

\author{
V S V Prabhakar ${ }^{1}$, K V K V L Pavan Kumar ${ }^{2}$ \\ ${ }^{1}$ Department of ECE, Koneru Lakshmaiah Education Foundation, \\ Green Fields, Vaddeswaram, A.P., India-522502. \\ E-mail: prabhakarvsv@kluniversity.in.
}

\begin{abstract}
:
This paper encases a $2 \mathrm{~T}$ MUX to decrease the semiconductor include in single piece viper component to 6. It utilizes HEP-1 apparatus in Coach GRAPHICS to think about boundaries like Power, delay, PDP, and ADP with 8TEXOR, 8TMUX, 6T viper and so on with $1.2 \mathrm{~V}$ gracefully voltage in 130-nm innovation. The proposed one presents an improvement of 51\%, 98\% in ADP and PDP individually when contrasted with the 8T MUX based Single piece viper component. Further, it underlines low force dispersal and least postpone required to show the ideal rationale that is helpful in numerous versatile applications.
\end{abstract}

Index Terms - full adder, power, delay, power delay product. 


\title{
Analysis and Application of Python on Machine Learning
}

\author{
V S V Prabhakar', K V K V L Pavan Kumar², K Praneeth \\ ${ }^{1,2}$ Department of ECE, Koneru Lakshmaiah Education Foundation, \\ Green Fields, Vaddeswaram, A.P., India-522502. \\ E-mail: prabhakarvsv@kluniversity.in.
}

\begin{abstract}
:
The Machine learning domain has developed rapidly the most recent couple of years, specifically in the Portable eHealth area. With regards to the D1NAMO venture, we meant to recognize hypoglycemia on Type 1 diabetes sufferers through utilizing their ECG, recorded with a game like achest belt. To know whether the information contains enough data for this characterization task, we expected to apply and assess AI calculations on a few sorts of highlights. We have assembled a python tool kit consequently. It is based over the scikit-learn tool stash and it permits assessing a characterized set of highlights extractors, dealing with applying great AI system, for example, crossapproval or parameters network search.
\end{abstract}

Index Terms - data acquisition, data preprocessing, python, machine learning. 


\title{
ROAC: Recursive Optimization of Ant Colony Assisted Perturb and Observe for a Photo Voltaic Resonant Boost Converter
}

\author{
Selvakumar $\mathbf{R}^{1}$, M Sujatha ${ }^{2}$, S Palanikumar ${ }^{3}$ \\ 1,2, Department of ECE, Koneru Lakshmaiah Education Foundation, \\ Vaddeswaram, A.P., India-522502. \\ E-mail: selvakumar.r@kluniversity.in
}

\begin{abstract}
:
This paper introduces a new Hybrid MPPT algorithm by combining new Ant Colony Optimization (ACO) and Perturb \& Observe (P\&O) method. The maximum power from a solar panel is extracted from all conditions like solar irradiance variation, temperature variation and partial shading conditions. Ant Colony Optimization (ACO) method tracks maximum power from panel under all variations and Perturb \& Observe algorithm used in final stage to achieve faster MPP tracking. This proposed algorithm is implemented both in Simulink and hardware. A 5kWp grid connected solar photovoltaic power plant is designed and implemented for the 15 stage 31 level Cascaded Multilevel Inverter (CMLI) with the Selective harmonic elimination algorithm. From the analysis of results, it is found that the proposed hybrid MPPT provides higher MPP tracking performance in any weather conditions compared with other MPPT algorithms.
\end{abstract}

Index Terms - Ant Colony Optimization, Cascaded Multilevel Inverter, Selective harmonic elimination algorithm. 


\title{
Design and optimization of single photon indium phosphide on silicon chip for FMCW LiDAR
}

\author{
Selvakumar $\mathbf{R}^{1}$, M Sujatha ${ }^{2}$, S Palanikumar ${ }^{3}$ \\ 1,2Department of ECE, Koneru Lakshmaiah Education Foundation, \\ Vaddeswaram, A.P., India-522502. \\ E-mail: selvakumar.r@kluniversity.in
}

\begin{abstract}
:
Text Single Photon (SP) driven silicon chip for autonomous System (AS) are the present era in automation sector. We proposed an Indium Phosphide (InP) material composite design and optimization to improve the photon mobility, conductivity and frequency. Material used for fabrication of autonomous system performance is directly propositional in the following factors: thermal stability in various refractive index $(\mathrm{nD})$ The proposed material InP integrated with the white phosphorus reaction with indium iodide at $400{ }^{\circ} \mathrm{C}$, it induces the direct band gap $0.9366 \mathrm{eV}$ (300 and speed of electron moments scattered in all the areas. To make it intense path instead of electron we introduced single photon indium phosphide (SPInP) on silicon chip (SoC) adding with photonic integrated circuits (PICs). In this method wavelength sensitivity improved single to three pulse range various from $905 \mathrm{~nm}$ to $1550 \mathrm{~nm}$ compound attenuation reaches to $0.36666 \mathrm{~dB} / \mathrm{Km}$. Signal and detection and conversion SPInP SoC more suitably in Frequency Modulated Continuous Wave (FMCW) coherent based Light Detection and Ranging (LiDAR) integrated with Indium Phosphide photomultipliers (InPPMs) theory parameter analysis, optimized calculation of Signal to Noise Ratio (SNR), Peak to Average Power ratio (PAPR), Angle of Arrival (AoA), Distance, velocity, error detection in frequency domain and simulation desired characteristics are presented.
\end{abstract}

Index Terms - Autonomous System, silicon on chip, refractive index. 


\title{
Implementation of Transform Coding Through C Based Vivado HLS
}

\author{
P. Srikanth Reddy ${ }^{1,}$ K.V.K.V.L. Pavan Kumar ${ }^{2}$ \\ ${ }^{1,2}$ Department of ECE, Koneru Lakshmaiah Education Foundation, \\ Vaddeswaram, A.P., India-522502. \\ E-mail: palagani.srikanth@kluniversity.in, pavan.cec@kluniversity.in
}

\begin{abstract}
:
This paper presents a methodology for equipment execution of 8X8 DCT(discrete cosine change) and IDCT(inverse discrete cosine change) through HLS.DCT is a oftentimes utilized calculation for picture pressure in HEVC,XVID CODECS.DCT/IDCT are commonly actualized through HDL approach, yet the execution through HDL is a period taking measure and has higher computational unpredictability. In this paper a 8X8 DCT\&IDCT are actualized through HLS alongside HDL. HLS is another rising system which is used when there is a necessity for quicker plan and execution of equipment modules. In this paper a usage of 8X8 DCT and IDCT through CHEN-WANG calculation in HLS is moreover exhibited which is utilized in XVID CODECS. The outcomes acquired are contrasted and HDL, which shows that HLS gives same outcome as HDL approach. However, HLS contrasted and HDL has restriction regarding asset allotment however gives simpler usage regarding plan, confirmation time and computational multifaceted nature.
\end{abstract}

Index Terms - DCT,CODEC,IDCT,HDL 


\title{
High Performance 2D Transform using Area Efficient Methodology for Future Video Coding P. Srikanth Reddy ${ }^{1}$, K.V.K.V.L. Pavan Kumar ${ }^{2}$
}

\author{
${ }^{1,2}$ Department of ECE, Koneru Lakshmaiah Education Foundation, \\ Vaddeswaram, A.P., India-522502. \\ E-mail: palagani.srikanth@kluniversity.in, pavan.cec@kluniversity.in
}

\begin{abstract}
:
Future Video Coding(F.V.C),High Efficiency Video Coding(H.E.V.C) are worldwide picture pressure principles created by ITU,ISO,JPEG associations to deliver better pressure factor to a detriment of high computational complexity.FVC has higher computational multifaceted nature and asset use contrasted with HEVC, H.264 standards.FVC uses different DCT calculations for picture pressure and different IDCT calculations for picture remaking. This paper presents a methodology for Hardware execution of 8X8 DCT, IDCT modules in Design1 through HDL (Verilog).Design 1 can create 64 Transformed Coefficients for each cycle. This execution uses equipment assets (multipliers, adders) at higher expense.Inorder to conquer this issue a methodologyhasbeenimplementedinDesign2throughevenodd decay calculation. Plan 2 can produce 64 change coefficients per clock cycle with less use of multipliers contrasted with Design 1.Multipliers involve more territory in equipment executions. This paper predominantly focusses to decrease equipment assets however much as could be expected. To dispose of use of multipliers totally a strategy has been proposed in this paper. The proposed strategy has been executed in Plan 3 creates 8 change coefficients for every cycle with complete disposal of multipliers (With Zero (0)multipliers).Design 3 additionally diminishes Four(4) phases of DCT,IDCT operations to Two(2)stages which reduces the number of change coefficients to be used. This alteration diminishes the adders and shifters tally to negligible number. Anyway this usage produces 64 change coefficients after 8 clock cycles.All the Designs are simulated and synthesized using Xilinx Vivado(2018.1). The results obtained arecomparedin terms of both reproduction and blend shows that the Proposed Methodology.
\end{abstract}

Index Terms -DCT,IDCT,Multiplier,HDL. 


\title{
Analysis of an Energy-Balanced Underwater Acoustic Sensor Networks
}

\author{
V. Teju ${ }^{1,}$ K.V. Sowmya ${ }^{2,}$ N.V.Satyanarayana Murthy ${ }^{3,}$ \\ M.LakshmiYamini $^{4}$, K. SaiGopinath ${ }^{5}$ \\ 1,2,3,4,5 Department of ECE, Koneru Lakshmaiah Education Foundation, \\ Green Fields, Vaddeswaram, A.P., India-522502.

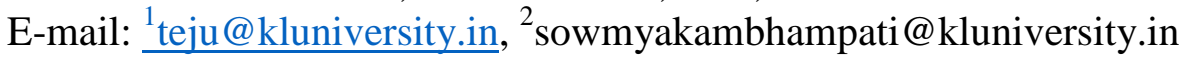

\begin{abstract}
:
Underwater Wireless Sensor Networks (UWSNs) have drawn tremendous concerns from all fields in mild in their huge application. Submerged remote sensor systems are like earthly wireless Sensor Networks (WSNs), be that as it is able to, due to numerous place of job and correspondence medium, UWSNs have several novel attributes, as an instance, excessive piece mistake price, lengthy begin to complete defer and coffee transfer pace. Those attributes of UWSNs purpose several troubles, for instance, retransmission, high power utilization and coffee dependability. To cope with those issues, many directing conventions for UWSNs are proposed. On this paper, an issue free steering convention, named power green directing convention depending on layers and inconsistent bunches (EERBLC) is proposed. EERBLC conference comprises of 3 ranges: layer and inconsistent agency association, transmission directing, maintenance and replace of bunches. Within the essential degree, the gazing zone under the water is partitioned into layers; the hubs in a comparable layer are bunched. For adjusting strength of the whole device and keeping far away from the "hotspot" difficulty, a unique inconsistent bunching approach depending on layers for UWSNs is proposed, wherein any other figuring technique for inconsistent institution length is exhibited. Inside the period in-between, every other bunch head dedication machine relying on power parity and degree is given. In the transmission degree, EERBLC convention proposes completely unique next forwarder strength of will technique depending on the sending percent and the lingering energy.
\end{abstract}

Index Terms-: UWSN, EERBLC 


\title{
Implementation of Real Time Moving Object Detection Using Region-Based Fully CNN
}

\author{
V. Teju' K.V. Sowmya ${ }^{2,}$ N.V.Satyanarayana \\ Murthy $^{3,}$ M.LakshmiYamini, ${ }^{4}$ K. SaiGopinath ${ }^{5}$ \\ 1,2,3,4,5 Department of ECE, Koneru Lakshmaiah Education Foundation, \\ Green Fields, Vaddeswaram, A.P., India-522502. \\ E-mail: ${ }^{1}$ teju@kluniversity.in, ${ }^{2}$ sowmyakambhampati@kluniversity.in
}

\begin{abstract}
:
Vision systems are essential in building a mobile robot that will complete a certain task like navigation, surveillance, and explosive ordnance disposal (EOD). This will make the robot controller or the operator aware what is in the environment and perform the next tasks. With the recent advancement in deep neural networks in image processing, classifying, and detecting the object accurately is now possible. In this paper, Convolutional Neural Networks (CNN) is used to detect objects in the environment. Two state of the art models are compared for object detection, Single Shot Multi-Box Detector (SSD) with MobileNetV1 and a Faster Region-based Convolutional Neural Network (Faster-RCNN) with InceptionV2. Result shows that one modelis ideal for real-time application because of speed and the other can be used for more accurate object detection.
\end{abstract}

Index Terms - RF-CNN, SSD 


\title{
An Efficient Compression for ECG Signal using SPIHT Algorithm and Classification Using Fuzzy SVM System
}

\author{
R. Sekar \\ Department of ECE, Koneru Lakshmaiah Education Foundation, \\ Vaddeswaram, A.P., India-522502. \\ E-mail: shekharmohith09@kluniversity.in
}

\begin{abstract}
:
Wireless Body sensor networks enabled electrocardiogram (ECG) biosensors are a novel solution for patient centric telecardiology. The energy efficiency of the present wireless ECG biosensors still needs to be improved. In this paper an efficient dynamic compression scheme is proposed to deal with the challenge of ultralow power and real-time ECG application. I propose the novelty algorithm is IF sampler based SPIHT algorithm. The SPIHT method is based on the use of wavelet transform which is very well suited to energy of the signal in fewer coefficients. The FUZZY based SVM classifier results in automatic sleep stage classification and arrhythmia classification based on ECG signal. Beat reordering rearranges beat order in 2D ECG array based on the similarity between the adjacent beats. The experimental results show that the high compression ratio, low reconstruction errors, accuracy of sleep stage and arrhythmia classification. The proposed method preserves signal characteristics for the automatic sleep stage and arrhythmia classification problems and SPIHT based ECG compression implies the lossless compression.
\end{abstract}

\section{Index Terms -SPIHT algorithm, ECG, Compression.}




\title{
Efficient Estimation and Removal of Noise Parameters from Current Read Out Digital Image Sensor using Variance Transforms
}

\author{
R. Sekar \\ Department of ECE, Koneru Lakshmaiah Education Foundation, \\ Vaddeswaram, A.P., India-522502. \\ E-mail: shekharmohith09@kluniversity.in
}

\begin{abstract}
:
Digital Remote Image (DRI) denoising using thresholding methods means find appropriate value (threshold) which separates noise values to actual image values without affecting the significant features of the image. The aim of this project is to develop a best poissons noise removal filter from the comparative study analysis of filtering methods and successive approximation based thresholding technique. To estimation Poisson noise this is de-noised using successive approximation and filtering techniques. First the noise is removed by median filter and then removed by wiener filter. Second noisy image is denoised with the help of wavelet based techniques using thresholding Third thresholding is applied on the result of first and second simultaneously for image denoising and fourth PSNR (Peak Signal to Noise Ratio), MSE (Mean Square Error) calculated and results are compared in all cases.
\end{abstract}

\section{Index Terms -Digital Remote Image, Thresholding, PSNR}




\title{
Design of Nanoscale Square Ring Resonator BPF Using MIM Waveguide
}

\author{
Surendra Kumar Bitra ${ }^{1}$, Sridhar $\mathbf{M}^{2}$ \\ ${ }^{1}$ Research scholar, Dept of ECE, KLEF, \\ Vaddeswaram, Andhra Pradesh, India. \\ ${ }^{2}$ Professor, Dept of ECE, Vaddeswaram, Andhra Pradesh, India. \\ E-mail: bitrasurendrakumar@gmail.com ${ }^{1}$, \\ sridhar.m@kluniversity.in ${ }^{2}$
}

\begin{abstract}
:
In this Work, Metal-Insulator-Metal (MIM) based single band plasmonic bandpass filters (BPF) design and analysis is presented. The basic design comprises of a Square Ring Resonators (SRR) is coupled using straight wave-guide with gap. The straight waveguide SRR is responsible for a single band operating wavelength (1355 nm, i.e. $221.24 \mathrm{THz})$. Design and simulations are carried out using Finite Difference Time Domain (FDTD) based solver embedded in the Computer Simulation Technology (CST) Microwave Studio suite. The pro-posed filters are used for plasmonic single band bandpass filter (BPF) applications in photonic integrated circuits (PIC's).
\end{abstract}

Index Terms -MIM, Photonic Integrated Circuits, SRR and BPF. 


\title{
Reduction of Peak to Average Power Ratio in OFDM System by Using Selected Mapping Technique
}

\author{
T.Penchala Naidu ${ }^{1}$, K.V.K.V.L. Pavan Kumar ${ }^{2}$ \\ Department of ECE, Koneru Lakshmaiah Education Foundation, \\ Vaddeswaram, A.P., India-522502. \\ E-mail: penchalanaidu@kluniversity.in
}

\begin{abstract}
:
The primary disadvantages of OFDM are high PAPR and between image impedance. The chose planning procedure is one of the most famous PAPR decrease plans for the OFDM framework. The current plan, which is utilized to decrease PAPR, is separating. In Filtering plan, there might be a chance of sign misfortune. So the separating strategy is certifiably not a best procedure to lessen PAPR in OFDM framework. Another procedure used to decrease PAPR is cutting. In the cut-out cycle, the first sign might be lost. The computational multifaceted nature of both separating and cutting is less, yet generally speaking correspondence framework execution will endure with the utilization of these strategies because of the loss of wanted sign. Presently the proposed procedure is SLM. In the SLM procedure, the regulated data is duplicated with various stage arrangements lastly we select the increased stage information with negligible PAPR. The OFDM framework with SLM is to some degree complex however the estimation of PAPR will be decreased. The other huge disadvantage of the OFDM framework is ISI. The ISI is there due to the multipath impact. To diminish the ISI content, we are utilizing the RAKE recipient. The beneficiary is planned so that it gets all the multipath signals, at that point does the time scaling to arrange all the multipath signals, lastly midpoints all the time-scaled multipath signals. The proposed method is SLM. The computational unpredictability of translating OFDM with SLM is substantially less than that of OFDM framework. The recreation results show the PAPR decrease execution.
\end{abstract}

Index Terms -OFDM, PAPR, ISI, SLM. 


\title{
Transition Analysis of Beam Scintillation in R-FSOC Links Using Discrete Markov Chain Model
}

\author{
Rahul Bose \\ Department of ECE, Koneru Lakshmaiah Education Foundation, \\ Vaddeswaram, A.P., India-522502. \\ E-mail: rahulbose@kluniversity.in
}

\begin{abstract}
:
The intent of this chapter is to estimate the turbulence inflicted instantaneous beam scintillation level in an optical reflector-assisted FSO link by incorporating a CSI estimation technique that does not require the knowledge of the power of the optical beam at the Tx. The concept of the propounded CSI determination technique is then illustrated by estimating the optical scintillation level in an OOK based R-FSOC test bed. A FSMC model is then developed for analyzing the unconditional and stationary transitions in the laser scintillation level in the R-FSOC test bed. Discrete turbulence states are first perceived in the non-uniform temperature induced turbulent regimes. Thereafter, the BER performance of the CSI based R-FSOC system is analyzed to demarcate the SNR admissible region for each turbulence state. By comparing the instantaneous SNR of the received signal with the SNR admissible region, the transition probability of the scintillation process to all the possible transition states are evaluated, and the O-TSTP matrix is developed for the artificially fostered turbulent channel. By incorporating the Eigen value and limiting probability analysis on the O-TSTP matrix, the FSMC mixing time and the scintillation rates for the experimental link are estimated to be $3.71 \mathrm{~s}$ and $4.09 \%$ respectively.
\end{abstract}

Index Terms - CSI, FSMC, SNR 


\title{
Analytical Tracking of Beam Fluctuations in Random Media
}

\author{
Rahul Bose \\ Department of ECE, Koneru Lakshmaiah Education Foundation, \\ Vaddeswaram, A.P., India-522502. \\ E-mail: rahulbose@kluniversity.in
}

\begin{abstract}
:
This paper demonstrates the implementation of specific perturbation techniques to analytically track the distortions in the optical beam propagation in the linear and non-linear media with fluctuating atmospheric refractive indices. The order of the ODE that characterizes the optical field propagation is not affected by the perturbed RI. The asymptotic solutions generated through RP method comprises of secular terms that result in tracking error in proportion to the beam propagation distance. The negative effects of the secular terms are significantly reduced by: (a) obtain CFs with HOTs, or (b) employing PL perturbation technique. The asymptotic solutions generated through RP and PL perturbation methods are observed to trail the optical fluctuations with minimal tracking error. The RP technique is incorporated in for deriving the instantaneous CSI in order to gauge the turbulence induced scintillation level across a reflector assisted FSOC link without the knowledge of the transmitted beam power.
\end{abstract}

\section{Index Terms -Perturbation technique, RP technique, FSOC link}




\title{
Detection of Objects for Autonomous Cars Using Lane Detection Method
}

\author{
A.Poorna Priya \\ Department of ECE, Koneru Lakshmaiah Education Foundation, \\ Vaddeswaram, A.P., India-522502. \\ E-mail: putchapoornapriya @kluniversity.in
}

\begin{abstract}
:
This paper presents two algorithms, one to detect, track objects and the other for lane line detection. This detection and tracking of objects are done by using a Tensor flow object detection API. And a simple computer vision technique is obtained for Lane line detection. The objects Location which are identified that is forwarded to the algorithm of object detection and tracking. The object detection tracking system algorithm is used for obtain by using CVV. The proposed approach can able to detect objects in different illumination and occlusion. It accuracy of achieved is $90.88 \%$ on self-generated image sequences.
\end{abstract}

\section{Index Terms -object detection, CVV.}




\title{
Design of Birds Detector and Repellent Using Frequency Based Repeller by Machine Learning Algorithm
}

\author{
A.Poorna Priya \\ Department of ECE, Koneru Lakshmaiah Education Foundation, \\ Vaddeswaram, A.P., India-522502. \\ E-mail: putchapoornapriya@kluniversity.in
}

\begin{abstract}
:
Among entire globe many Countries people work as farmers to survive their lives . Plants are interconnected to farming and pests which are related to plants, where there are bulk of plants then they lead to farming there must be effect causing animals called pests. Pests are not only animals they may be birds too that damage the plants. One of the example of pests are birds and monkeys that eat plants and its products and cause harm to farmers. In This research technique we has developed and designed a prototype of birds detector and repellent of birds based on frequency. This designed model has a Bird detection and repellent system which detects the bird based on its given python code as well as with the help of some machine learning algorithms and then proceeds further step to a repellent which produces a certain frequency with respective the birds by classificating the birds based on there resonating frequencies, so the bird will be disturbed through the repellent frequency and goes away fron it. This designed model consists of python open cv software as a detector to detect movement from birds and further classifies the type of bird and for that type it give signals to further step to fire certain signal frequency to repeller .The repeller is designed using LC oscillator type colpitts with Piezo Ultrasonic sensor as repeller which produces . The obtained results of the experiment are mainly focussed on Birds with bird detection system, which is capable of working with a detection distance between $2 \mathrm{~m}-500 \mathrm{~m}$ by using sensors to detect bird, and by modifying this model in aspect of sensor we used machine learning algorithm of python open $\mathrm{cv}$ it increases the range of detectiong the bird from $500 \mathrm{~cm}$ to 500 meters and can continue for further process to repel the birds at a frequency signal of about $60 \mathrm{kHz}$.
\end{abstract}

\section{Index Terms -Bird detection, repeller, sensor}




\title{
A Novel Method to Detect OSA Using Deep Convolution Neural Network
}

\author{
S.Nagendram \\ Department of ECE, Koneru Lakshmaiah Education Foundation, \\ Vaddeswaram, A.P., India-522502. \\ E-mail: nagendram@kluniversity.in
}

\begin{abstract}
:
Obstructive sleep apnea (OSA) is a general problem where individuals breathing are disturbed in the sleep. Major sleep apnea issues are presently unidentified because of cost and real time limitations of overnight poly so monography (PSG) at sleep experiments, where an expert professional monitoring is required to work over night. Recent techniques for sleep apnea classification are being designed by bioengineers for most feasible and accurate detection. In this paper, a classification algorithm automatically processes short intervals epochs of the electrocardiogram (ECG) input. Using support vector machines (SVM), the classification algorithm automatically trains and tests the data on subjects with and without OSA. One of the new approaches for this study is obtaining the information from single channel ECG recordings for the detection of OSA. The present work contributes the development of diagnosing the Sleep Apnea based on Machine Learning algorithms. This ML based detection helps in consuming less time and with the usage of this technique helps in improving its efficiency.
\end{abstract}

\section{Index Terms -Obstructive sleep apnea, electrocardiogram.}




\title{
Analytics models for Diabetes Prediction using a Machine Learning
}

\author{
S.Nagendram \\ Department of ECE, Koneru Lakshmaiah Education Foundation, \\ Vaddeswaram, A.P., India-522502. \\ E-mail: nagendram@kluniversity.in
}

\begin{abstract}
:
Diabetes is one of the essential illnesses everywhere at some degree inside the area. Consistent with the International Diabetes Federation (IDF) document of the three hundred and sixty five days 2017 , diabetes isn't abnormal in greater or less eight. $8 \%$ of the Indian person human beings and is one of the incredible ten perception techniques in destruction in India. In untreated and unidentified diabetes should reason adjustments within the sugar tiers and silly occurrences, damage organs, as an instance, kidneys, eyes, and veins in the coronary heart. Via using device gaining knowledge of estimations to anticipate the infection from the applicable datasets at a beginning time need to extra then in all likelihood extra human lives.
\end{abstract}

\section{Index Terms -perception techniques, coronary.}




\title{
International Journal for Innovative
} Engineering and Management Research

A Peer Revieved Open Access International Journal

\section{An IOT Based Multiform Cooling System with Security}

\author{
Musala Venkateswara Rao \\ Department of ECE, Koneru Lakshmaiah Education Foundation, \\ Green Fields, Vaddeswaram, A.P., India-522502. \\ E-mail: venkatvlsi@kluniversity.in.
}

\begin{abstract}
:
Nowadays power optimization is crucial in-home automation and industrial applications. Air conditioners (AC) are extensively used in residential, shopping malls and commercial buildings, which consumes more power in the total consumption of the year. An efficient smart air conditioning system is required to address all these problems and efficient usage of the power based on the needfulness. In this paper we are proposing Smart Air Conditioning Monitoring System (SACMS) based on Internet of Things (IOT). At present our model is developed for a group of conference halls with a common cooling system, this purpose can be accomplished by counting the number of people in that building so that we can control the weather conditions in certain building premises according to the necessary density. The main objective is to control the air conditioning temperature based on object counter mechanism using Object Detection Sensor (ODS). Object counting system is the method in which the number of persons entering and leaving the hall is counted and the temperature inside the room is maintained accordingly. This system uses the help of open source firmware named NODE MCU which is used to store data. This device can be used to conserve energy by shutting off air conditioning automatically when no person is present in the room.
\end{abstract}

Index terms: Internet of Things (IOT), Security, Air Conditioning, Smart Power, Object Detection. 


\title{
Hand-drawn Circuit Recognition with Deep Learning
}

\section{Musala Venkateswara Rao}

\author{
Department of ECE, Koneru Lakshmaiah Education Foundation, \\ Green Fields, Vaddeswaram, A.P., India-522502. \\ E-mail: venkatvlsi@kluniversity.in.
}

\begin{abstract}
:
In electrical science, a circuit outline is a graphical depiction of an electrical circuit that contains fundamental picture to address the electrical fragments and accessibility between parts. For example, a resistor in an electrical diagram has a value and two relationship with various parts. People can see these electrical parts using their understanding which they recently arranged. Starting there, it is relied upon to truly enter the portions into machine to play out the related strategy even the graph is puzzled with massive amounts of fragments. This procedure is not viable if there ought to emerge an event of unusualness of the graphs. In this paper we proposed another circuit confirmation technique in which the machine can coordinate examine a bewildering circuit outline. The Combination of various component symbols and their connections gives an Electrical Circuit. Here we present an image segmentation technique which perceives the hand-drawn circuit diagrams. The picture which is drawn by hand will be examined and that filtered picture of the hand-drawn circuit chart will be pre-prepared so we can expel the un-needed pieces of the graph and convert that into bilevel. An electrical circuit is generally made from portion pictures and their affiliations. This endeavor proposes a structure for modified affirmation of hand-drawn electrical circuits subject to picture division and game plan figuring's. Each part picture is expelled from affiliation wires, by then a distinctive verification method is applied to each part of the image. The yield of system is a netlist that perceives the picture types and interconnections between them. The netlist can be used to make PC schematic of circuit.
\end{abstract}

Index Terms -Graphical Depiction, Image Segmentation, Scanned Image, Electrical Circuit, Netlist, PC Schematic. 


\title{
Audio Denoising by Time-Frequency Block Thresholding
}

\author{
M. Venu Gopala Rao ${ }^{1}$, Ms. Harsha Mane ${ }^{1}$ \\ ${ }^{1}$ Department of ECE, Koneru Lakshmaiah Education Foundation, \\ Green Fields, Vaddeswaram, A.P., India-522502. \\ E-mail: mvgr03@kluniversity.in.
}

\begin{abstract}
:
Audio signals are often contaminated by background environment noise and buzzing orhumming noise from audio equipments. Audio denoising aims at attenuating the noise while retaining the underlying signals. Applications such as music and speech restoration are numerous. Removing noise from audio signals requires a non-diagonal processing of time-frequency coefficients to avoid producing "musical noise". State of the art algorithms perform a parameterized filtering of spectrogram coefficients with empirically fixed parameters. Diagonal time-frequency audio denoising algorithms attenuate the noise by processing each window Fourier or wavelet coefficient independently, with empirical Wiener, power subtraction or thresholding operators. These algorithms create isolated time-frequency structures that are perceived as a "musical noise". The musical noise is strongly attenuated with non-diagonal time-frequency estimators that regularize the estimation by recursively aggregating time-frequency coefficients. We propose a new nondiagonal audio denoising algorithm through adaptive time-frequency block thresholding, which adjusts all parameters adaptively to signal property by minimizing a Stein estimation of the risk. Numerical experiments demonstrate the performance and robustness of this procedure through objective and subjective evaluations.
\end{abstract}

Index Terms - Audio denoising, Block thresholding, Ephraim and Malah, Power spectrum, Power subtraction, Thresholding 


\title{
Automatic Speech Recognition in Meeting Rooms
}

\author{
M. Venu Gopala Rao ${ }^{1}$, Ms. Harsha Mane ${ }^{1}$ \\ ${ }^{1}$ Department of ECE, Koneru Lakshmaiah Education Foundation, \\ Green Fields, Vaddeswaram, A.P., India-522502. \\ E-mail: mvgr03@kluniversity.in.
}

\begin{abstract}
:
Performance of current speech recognition systems severely degrades in the presence of noise and reverberation. While rather simple and effective noise reduction techniques have been extensively applied, coping with reverberation still remains as one of the toughest problems in speech recognition and signal processing. Single-microphone dereverberation algorithms typically result in very low speech recognition performance. Taking advantage of multiple-microphones popularity and theoretical achievements such as the MINT theorem, blind multiple-microphone signal processing techniques are explored and evaluated in several speech recognition test-beds. These include connected digits recognition in meeting rooms and matched and mismatched training-test conditions as well as conversational speech recognition in meetings in mismatched training-test conditions. Multi-channel equalization techniques are shown to be effective under explicit knowledge of either speaker-to-receiver impulse response or order determination, but not in real situations. Blind dereverberation techniques based on time-delay estimation, such as delayand-sum, delay-and-feature-domain-sum and phase-error based filtering are shown to significantly improve recognition accuracy over a single distant micro-phone, while being robust enough for real noisy and reverberant speech. Dereverberation techniques based on linear prediction are introduced and multi-channel correlation shaping is further explored for speech recognition. It is shown to improve recognition accuracy of a single distant microphone only in some situations.
\end{abstract}

\section{Index Terms - Speech recognition, dereverberation, equalization, multichannel,}




\title{
Effective Microstrip Feed Line Length For Ultra-Wide Band Responses and Wireless Applications
}

\author{
Mohan K N ${ }^{1}$, K Himaja Reddy ${ }^{2}$, Kannadassan ${ }^{3}$, \\ Jagadish Chandra Mudiganti ${ }^{4}$ \\ ${ }^{1}$ Department of ECE, Koneru Lakshmaiah Education Foundation, \\ Vaddeswaram, A.P., India-522502. \\ E-mail: knmohan@kluniversity.in \\ ${ }^{2}$ Vellore Institute of Technology, Vellore, \\ Tamilnadu India \\ ${ }^{3}$ Vellore Institute of Technology, Amaravti, AP, \\ India
}

\begin{abstract}
:
In this article, the effected feed line techniques used instead of DGS (Defected Ground Structure) and CSRR (Coupling Split Ring Resonator) which are commonly used for achieving UWB (Ultra-Wideband Applications) response. The classical antenna designed for $2.4 \mathrm{GHz}$ frequency and feed line used for this patch antenna has designed to $180^{\circ}$ electrical length. The resonance of antenna responses as UWB and it has interesting current distribution through the patch antenna; this paper avoided all aspects of techniques which are proposed to achieve UWB.
\end{abstract}

Index Terms -UWB, CSRR, DGS, Microstrip patch antenna. 


\title{
Distributed Peer-To-Peer Data Storage and Synchronizing Websites
}

\author{
Mohan K N1, T. Maneesh Reddy 2, K. Sai Charan 3, A. Ganesh 4 \\ ${ }^{1}$ Department of ECE, Koneru Lakshmaiah Education Foundation, \\ Vaddeswaram, A.P., India-522502. \\ E-mail: knmohan@kluniversity.in
}

\begin{abstract}
:
In Peer-to-Peer file distributing systems, content duplication technology is widely accustomed to scale down hot-spots and improve file requesting efficiency. Today's Internet architecture is designed based on the Client-Server model, which establishes lots of challenges. By proposing the protocol of the Peer-to-Peer file duplication application, these challenges will be resolved resourcefully. However, the impact of this protocol will only be in pinnacle when the contributed systems are elevated. With this protocol when implemented in the application of websites then the data - such as website content (back- end, front-end files) can be stored and shared by different individuals whoever visits the website. Additionally, using Bitcoin Cryptography and Secure Hash Algorithm (SHA) to encrypt the files and avoid tampering by other peers. For the discovery of peers involved, the BitTorrent Protocol is applied. This Peer-to-Peer file duplication protocol implementation will increase the availability of the website exponentially, concerning the number of peers involved in contributing to the website. In this paper we will execute a Decentralized Peer-to-Peer based application for file synchronizing and storing, using the Bitcoin Cryptography and BitTorrent Protocols.
\end{abstract}

Index Terms - BitTorrent protocol, Distributed storage, File synchronization. 


\title{
Brain Tumor Detection from MRI Images Using Optimization Segmentation Techniques
}

\author{
M. Anil Kumar ${ }^{1}$ \\ ${ }^{1}$ Department of ECE, Koneru Lakshmaiah Education Foundation, \\ Green Fields, Vaddeswaram, A.P., India-522502. \\ E-mail: anilkumarm@kluniversity.in
}

\begin{abstract}
:
The segmentation of medical images is a dynamic and relaxing task because of the distinct features of the images. It is one of the software needed for brain image segmentation to identify the brain tumor and will also be applied to the pre-processing stage to improve detection accuracy. Brain segmentation for magnetic resonance imaging (MRI) is a challenging task given the high-quality Picture quality, anatomical brain structures and tumor complexity. Several researchers have developed several algorithms around tumor segmentation in the field of medical imaging. This work contrasts the effects of optimization with Particle Swam Optimization (PSO) segmentation technique and Darwinian Particle Swam Optimization (DPSO) and Functional Order Darwinian Particle Swam Optimization (FO-DPSO). Using these three chosen methods, the accuracy of the collected data set has been measured and the findings are addressed, the paper from these. We validate these results with the PSO, DPSO and FOPSO, which are commonly used. The method used in the study of medical images. The future work will measure the size of the expected tumor area, which will give the algorithms more accuracy.
\end{abstract}

Index Terms -DPSO, PSO, FOPSO. 


\title{
IoT Based Reconfigurable Smart City Architecture
}

\author{
M. Anil Kumar \\ Department of ECE, Koneru Lakshmaiah Education Foundation, \\ Green Fields, Vaddeswaram, A.P., India-522502. \\ E-mail: anilkumarm@kluniversity.in
}

\begin{abstract}
:
Now-a-days Internet of Things is deployed almost in every application regarding smart cities and have been initiated to develop all over the world. Smart Devices are being geared up to ease human life. Due to the growth in Internet of Things technology, smart cities are been developed with this technology to work with the issues of public as well as private. The aim of the article is to enhance a solution to the problems in the smart cities with latest IoT architecture, protocols and services. With the technical support of IoT, using low power Wireless Sensor Networks (WSN) which is connected to transfer the data from M2M applications. In addition to the IoT, intelligent fea-tures are integrated with the help of Computer vision makes the technology more flexible. The goal of this article is to engrave the ser-vices to challenge the real time environment with low power synthesis technique. Only then, the smart city features are improved and serves the mankind with IoT technology.
\end{abstract}

\section{Index Terms -Smart Devices, IoT.}




\title{
Improvement of Image Reconstruction Using Multiscale AM- FM Methods
}

\author{
K.T.P.S Kumar ${ }^{1}$ \\ Department of ECE, Koneru Lakshmaiah Education Foundation, \\ Green Fields, Vaddeswaram, A.P., India-522502. \\ E-mail: satishkumar@kluniversity.in
}

\begin{abstract}
:
This paper discuss about new AM-FM methods for image reconstruction. This approach is based on 2 basic ideas: i) AM-FM Demodulation using new gabor filterbank ii) New accurate methods for instantaneous frequency (IF) estimation. This project includes quasi-eigen function approximation (QEA), quasi local method (QLM) and variable-spacing local linear phase (VS-LLP) methods for improved accuracy. The new VS-LLP method is a generalization of QEA method where we choose the best integer spacing between the samples to adapt as a function of frequency. We also introduce QLM method for IF and IA estimation. We present different noises (salt\&pepper, speckle, poisson, Gaussian) decompositions to show that the proposed methods can be used to reconstruct and analyze general images. However, each technique has different properties, making it more suited for specific applications and this technique is implemented by using MATLAB.
\end{abstract}

\section{Index Terms -Amplitude modulation Frequency modulation (AM-FM), Image reconstruction, Instantaneuos frequency, Multiscale}




\title{
Performance analysis of Absolute Deviation Filter for
}

\section{Removal of Impulse Noise}

\author{
K.T.P.S Kumar \\ Department of ECE, Koneru Lakshmaiah Education Foundation, \\ Green Fields, Vaddeswaram, A.P., India-522502. \\ E-mail: satishkumar@kluniversity.in
}

\begin{abstract}
:
This paper presents the process of removal of impulse noises using different median filters. This contains a vector median filter that includes a new mechanism for the detection of impulses in colour images prior to further processing operations. When compared to other filters, the proposed filter will be tested for images corrupted by two sided fixed impulse noise model, nothing but salt and pepper noise. If the central vector pixel in a kernel is found to be corrupted, it is replaced by the vector median of the kernel, else it is kept unchanged. The vector pixels in a specified window are ranked on the basis of sum of the distances between each vector pixel and the other vector pixels in the window. Since the detection mechanism uses absolute deviation values, the computational complexity is reduced. The results show a better performance of the proposed algorithm in lesser execution time as compared to a number of existing schemes for restoring colour images corrupted by impulse noise.
\end{abstract}

Index Terms -kernel; vector median filter; rank-conditioned vector median filter; rankconditioned and threshold vector median filter; centre-weighted vector median filter; two-sided fixed impulses noise model. 


\title{
An Investigation on the Impact of Machine Learning in Wireless Sensor Networks and Its Application Specific Challenges
}

\author{
K. $\operatorname{Praghash}^{1}$, T. Karthikeyan ${ }^{2}$, K. Suresh Kumar ${ }^{1}$, R. Sekar ${ }^{1}$, \\ R. Ramesh Kumar ${ }^{1}$, S. Arunmetha ${ }^{1}$ \\ ${ }^{1}$ Department of ECE, Koneru Lakshmaiah Education Foundation, Green Fields, Vaddeswaram, \\ A.P., India-522502. \\ ${ }^{2}$ Department of Electronics and Communication Engineering, \\ Annamacharya Institute of Technology and Sciences, Rajampet, AP, India. \\ E-mail: praghash@kluniversity.in.
}

\begin{abstract}
:
The importance of Machine Learning (ML) in advanced system technologies are proven in literature. This chapter investigates the role of ML in Wireless Sensor Networks and the challenges specific to its applications. We discuss the background literature of the renowned ML concepts and ML techniques. Further we distinguish the role of ML in WSN with detailed literature review. Subsequently, ML techniques for WSN are discussed from the literature. This chapter ends with the description of Functional and application specific challenges.
\end{abstract}

Index Terms - Machine Learning (ML), Wireless Sensor Networks (WSN), Application, Challenges. 


\title{
Nuclear structure of some even and odd nuclei using shell model calculations
}

\section{Bhopendra Singh ${ }^{1}$, S. Suman Rajest ${ }^{2}$, K. Praghash ${ }^{3}$, Uppalapati ${ }^{4}$, and Srilakshmi R. Regin}

\author{
${ }^{1}$ Amity University, Dubai. \\ ${ }^{2}$ Vels Institute of Science, Technology \& Advanced Studies (VISTAS), Chennai, Tamil Nadu, \\ India. \\ ${ }^{3}$ Department of ECE, Koneru Lakshmaiah Education Foundation, Green Fields, Vaddeswaram, \\ A.P., India-522502. \\ ${ }^{4}$ Department of CSE, VFSTR Deemed to be University, Vadlamudi, Guntur, India. \\ ${ }^{5}$ Adhiyamaan College of Engineering, Hosur, Tamil Nadu, India. \\ E-mail:praghash@kluniversity.in.
}

\begin{abstract}
:
In this work, we determined the electron dispersing structure factors, just as the vitality levels of certain cores. The computation of electron dispersing structure factors needs numerous issues to be remembered for request to make these figures attainable and quick in time in light of enormous measure of terms speak to arithmetic, quantum mechanical speculations, atomic shell model hypotheses and equations. In the current work, we examined the impacts of the higher setup outside the shell model space and the inactive center which included Tassie Model (TM) to discuss the Longitudinal C2 electron scattering form factors for the nuclei: ${ }^{116} \mathrm{Sn},{ }^{92} \mathrm{Mo},{ }^{90} \mathrm{Zr}$, ${ }^{39} \mathrm{~K}$ and ${ }^{32} \mathrm{~S}$, which calculated for nuclei under consideration, are compared with those of experimental data. The HO and SKX possibilities have been utilized to compute the wave elements of outspread single-molecule framework components. Some hypothetical vitality levels of the ${ }^{52} \mathrm{Cr},{ }^{32} \mathrm{~S}$ and ${ }^{181}$ Ta nuclei are calculated compared with their experimental data. The shell model for windows code NuShellX@MSU has been used in this study.
\end{abstract}

Index Terms - Nuclear structure model, Electron scattering, quantum mechanics, Atomic shell model, Dispersion form factors. 


\title{
International Journal for Innovative
} Engineering and Management Research

A Peer Revieved Open Access International Journal

\section{Fuzzy Utilization in Speech Recognition and its Different Application}

\author{
J. Bennilo Fernandes ${ }^{1}$, Kasiprasad Mannepalli ${ }^{1}$ \\ ${ }^{1}$ Department of ECE, Koneru Lakshmaiah Education Foundation, Green Fields, Vaddeswaram, \\ A.P., India-522502. \\ E-mail: bennij05@gmail.com.
}

\begin{abstract}
:
Talk affirmation is one among the basic zones in cutting edge talk process. The examination of talk affirmation may be a bit of an examination for "artificial intelligence" machines that may "hear" and "appreciate" the verbally communicated data. The customary ways for talk affirmation like HMM and DTW, are outrageously inconvenient and time excellent. As such formal Fuzzy justification may be an endeavor in cutting edge talk process for the convincing portrayal of talk affirmation in a couple of utilization. The approach masterminded in the midst of this paper streamlines the utilization of fuzzy in talk affirmation and make the data dealing with time shorter. The case considered in the midst of this paper is that the least mind boggling, i.e., the example of speaker dependence, little vocabulary and disconnected words. There are various spectral and common choices isolated from human talk. The present ways for tendency acknowledgment from voice use basically MFCC and Energy feature. This paper briefs an overview concerning the present work on talk feeling ID strong for completing more examination by feathery approach.
\end{abstract}

Index Terms - Emotional Speech Recognition, Fuzzy, HMM, NN Applications. 


\title{
Emotion Recognition Using KNN
}

\author{
Bennilo Fernandes.J ${ }^{1}$, Ch. Bhargavi ${ }^{1}$, S. Arshad ${ }^{1}$, G. Sai Kumar ${ }^{1}$, G. \\ Sandeep ${ }^{1}$ \\ ${ }^{1}$ Department of ECE, Koneru Lakshmaiah Education Foundation, Green Fields, Vaddeswaram, \\ A.P., India-522502. \\ E-mail: bennij05@gmail.com.
}

\begin{abstract}
:
The human speech will offer totally different styles of records regarding the speaker and speech. The emotional state recognition helps an individual to identify their health conditions and mental stress. In this paper different emotions like happy, angry, fear, sad \& normal is examined for recognition. With this approach, a new database is collected form 8 students in different combinations like male \& female and utilized for analysing. For any speech identification or recognition feature extraction and classification acts as the key factor, in this paper MFCC based Vector quantisation is used for extracting the features and KNN classification for recognition accuracy is utilised. From the feature extraction of MFCC, the simulation results shows $84.3 \%$ of overall accuracy $\&$ in which emotional state like - Normall gives high recognition rate of $90 \%$.
\end{abstract}

Index Terms - Speech Recognition, MFCC, Vector Quantization, KNN. 


\title{
Design and Analysis of Split Ring Antenna with Frequency Reconfigurability LTE And WIMAX Applications
}

\author{
Pardhasaradhi $\mathbf{P}^{1}$, Madhav BTP' ${ }^{1}$, Prudhvi Nadh ${ }^{1}$, Anil Kumar ${ }^{1}$, Lakshman $^{1}$ \\ ${ }^{1}$ Department of ECE, Koneru Lakshmaiah Education Foundation, Green Fields, Vaddeswaram, \\ A.P., India-522502. \\ E-mail: pspokkunuri@kluniversity.in.
}

\begin{abstract}
:
This paper presents frequency reconfigurable double band antenna for WiMAX and LTE 2500 band applications utilizing four PIN diode switches. The antenna is conservative in size with measurements of $30 \times 30 \times 0.8 \mathrm{~mm} 3$ and planned on FR-4 dielectric substrate with a halfway ground plane. The created radio wire works in the frequency scope of LTE and WiMAX (2.5-2.69 $\mathrm{GHz}$ and 3.4-3.6 GHz) individually. The frequencies can be constrained by utilizing PIN diodes and reception apparatus achieved the addition going of 3.34-4.46 $\mathrm{dBi}$. This planned antenna resonating at 2.52 and $3.49 \mathrm{GHz}$ when the PIN diodes are in ON state and reverberating at 2.68 and $3.58 \mathrm{GHz}$ when PIN diodes are in OFF state. The proposed radio wire has bidirectional radiation at upper frequency groups and unidirectional at lower frequency groups. The proposed split ring organized reception apparatus has the radiation effectiveness of $94.12 \%$ at $2.52 \mathrm{GHz}$ and 90.34\% at $3.49 \mathrm{GHz}$ in $\mathrm{ON}$ state. Reception apparatus giving great arrangement between the deliberate (Antenna estimation arrangement with VNA) and recreated results (Ansys-HFSS).
\end{abstract}

Index Terms - LTE, WiMAX, Split ring structured antenna, radiation efficiency. 


\title{
Estimation of Statistical Parameters Using Image Analysis in Pure and Nano doped 60.08 LC Materials
}

\author{
Durga Prasad Tripathi ${ }^{1}$, P. Pardhasaradhi ${ }^{1}$ \& B. T. P. Madhav ${ }^{1}$ \\ ${ }^{1}$ Department of ECE, Koneru Lakshmaiah Education Foundation, Green Fields, Vaddeswaram, \\ A.P., India-522502. \\ E-mail: pspokkunuri@kluniversity.in.
}

\begin{abstract}
:
Synthesis, characterization and phase-transition studies in pure and $20 \mu \mathrm{l}$ citrate-capped Au nanodispersed liquid crystalline (LC) N-(p-nhexyloxybenzylidene)-p-n-octyloxy aniline, 60.08, compounds are done utilizing a polarizing magnifying instrument joined with hot stage and camera. The polarizing microscopy strategy is utilized to gauge the stage progress temperatures. Differential checking calorimetry is utilized to decide the progress temperatures and enthalpy esteems. By the scattering of citrate-covered Au nanoparticles into the LC mixes, the nematic change temperature is diminished. Moreover, factual boundaries-based picture improvement calculation is proposed to distinguish the glasslike stages in the unadulterated and nano-doped LC compound. Experimental outcome investigation plainly uncovers the vigor of this strategy over existing picture upgrade strategies.
\end{abstract}

Index Terms - Speech Recognition, MFCC, Vector Quantisation, KNN Differential scanning calorimetry; image enhancement; nanodispersion; statistical parameters. 


\title{
Design and Analysis of a Uniform Meander RF MEMS Switch
}

\author{
C Leela Mohan ${ }^{1}$, K Ch Sri Kavya ${ }^{1}$, K Sarat Kumar ${ }^{1}$ \\ ${ }^{1}$ Department of ECE, Koneru Lakshmaiah Education Foundation, Green Fields, Vaddeswaram, \\ A.P., India-522502. \\ E-mail: leelamohan416@gmail.com.
}

\begin{abstract}
:
This paper intended to design and analysis of uniform meander RF MEMS capacitive shunt switch. The low pull in voltage is obtained in flexure type membrane by proposed RF MEMS Switch. The materials selection for the beam and dielectric layer is described in this paper and also shown the performance depends on materials utilized for the design. The high isolation of $-31 \mathrm{~dB}$ actuating at the pull-in voltage of $11.97 \mathrm{~V}$ with a spring constant of $2.38 \mathrm{~N} / \mathrm{m}$ produced by the switch and is obtained by the optimization process.
\end{abstract}

Index Terms - RF MEMS, Meanders, Pull-In voltage, Switching time, Isolation Loss, Insertion Loss, Return Loss. 


\title{
Synthesis of PAA For SLL Reduction Using the Differential Evolution Algorithm
}

\author{
K. Ravi Tej ${ }^{1}$, K Ch Sri Kavya ${ }^{1}$, K Sarat Kumar ${ }^{1}$ \\ ${ }^{1}$ Department of ECE, Koneru Lakshmaiah Education Foundation, Green Fields, Vaddeswaram, \\ A.P., India-522502. \\ E-mail: ravi.tej489@gmail.com.
}

\begin{abstract}
:
In this paper, we synthesis an array consisting of uniformly excited unequally spaced elements for reducing SLLs. A globally optimal solution for the array elements phase and position is determined by using a differential evolutionary algorithm for a given radiation pattern. Reduced side-lobe levels are observed with position-phase synthesis. The obtained results are compared with the possible two cases i.e., uniform amplitude equal phase (variable position-control) and uniform amplitude unequal phase (variable phase-control). The obtained results justify that the positionphase synthesis technique is not only reduces the side-lobe levels to $(-25.28) \mathrm{dB}$, but also preserves the advantages of both variable position-controlled synthesis and variable phase-controlled synthesis with a minimum number of array elements.
\end{abstract}

Index Terms - Phased array, Uniform excitation, Side lobe level, Equal phase, Unequal spacing. 


\title{
Mathematical Modeling Approaches for Block chain Technology
}

\author{
K. SureshKumar ${ }^{1}$, M. Geetha ${ }^{2}$, Ch.Vidyadhari ${ }^{3}$ \\ ${ }^{1}$ Department of ECE, Koneru Lakshmaiah Education Foundation, Green Fields, Vaddeswaram, \\ A.P., India-522502. \\ ${ }^{2}$ Department of CSE, College of Engineering and Technology \\ ${ }^{3}$ Department of IT, SRM Institute of Science and Technology, Vadapalani, Chennai, Tamilnadu, \\ India. \\ E-mail: m.k.sureshkumar@kluniversity.in.
}

\begin{abstract}
:
These days blockchain innovation has become a significant issue as of late and greatly affected the business. Despite the fact that blockchain innovations may bring us reliable and security issues, advantageous administrations, and difficulties behind this procedure assumes a significant key job that we have to break down. To have a superior observation about this square chain innovation, we will find out about the essential security highlights like-expanded limit, better security, unchanging nature, quicker settlement and decentralized framework. The range of blockchain applications limits from budgetary, human services, vehicle, chance administration, Internet of things to open and social administrations. In this paper, by examining its structure to various agreement calculations just as difficulties and openings, we endeavor to lead an expansive going review on blockchain innovation and also the few mathematical models behind this blockchain technology.
\end{abstract}

Index Terms - Blockchain, Security, Privacy, Bitcoin, Smart contract. 


\title{
An Investigation on the Impact of Machine Learning in Wireless Sensor Networks and Its Application Specific Challenges
}

\author{
K. Praghash ${ }^{1}$, T. Karthikeyan ${ }^{2}$, K. SureshKumar ${ }^{1}$ \\ ${ }^{1}$ Department of ECE, Koneru Lakshmaiah Education Foundation, Green Fields, Vaddeswaram, \\ A.P., India-522502. \\ ${ }^{2}$ Department of Electronics and Communication Engineering, \\ Annamacharya Institute of Technology and Sciences, Rajampet, AP, India. \\ E-mail: m.k.sureshkumar@kluniversity.in.
}

\begin{abstract}
:
Abstract the importance of Machine Learning (ML) in advanced system technologies are proven in literature. This chapter investigates the role of ML in Wireless Sensor Networks and the challenges specific to its applications. We discuss the background literature of the renowned ML concepts and ML techniques. Further we distinguish the role of ML in WSN with detailed literature review. Subsequently, ML techniques for WSN are discussed from the literature. This chapter ends with the description of Functional and application specific challenges.
\end{abstract}

Index Terms - Machine Learning (ML) Application, Challenges, Wireless Sensor Networks (WSN). 


\section{Design of Two Stage CMOS Comparator with Improved Accuracy in Terms of Different Parameters}

\section{Shruti Suman}

${ }^{1}$ Department of ECE, Koneru Lakshmaiah Education Foundation,

Green Fields, Vaddeswaram, A.P., India-522502.

\section{ABSTRACT:}

E-mail: shrutisuman23@gmail.com

The well developing industry of electronics is insistent to low power and high speed and less area ADCs (analog to digital converters). Comparator is device that is especially employed in ADCs, used for division method, associated for square measure and chiefly liable for delay created and power consumption by an ADC. A low power and high-speed comparator are needed to satisfy the longer-term demands. The circuit conferred during this paper is designed using $0.35 \mu \mathrm{m}$ CMOS technology with $1.65 \mathrm{~V}$ bias voltage and $12 \mu \mathrm{A}$ bias current. Cadence virtuoso tool is employed for the designing and simulation for the comparator circuit. The correct analysis of propagation delay, settling time, speed of the comparator is mentioned very well in detail.

\section{Index Terms - CMOS Technology, high speed devices, comparator}




\title{
Two Stage CMOS Operational Amplifier: Analysis and Design
}

\author{
Shruti Suman \\ ${ }^{1}$ Department of ECE, Koneru Lakshmaiah Education Foundation, \\ Green Fields, Vaddeswaram, A.P., India-522502. \\ E-mail: shrutisuman23@gmail.com
}

\begin{abstract}
:
This paper describes analysis and design of 2-stage CMOS operational amplifier (Op Amp). The designed circuit operates at $3.3 \mathrm{~V}$ of supply voltage and at TSMC $0.35 \mu \mathrm{m}$ CMOS technology. The performance parameters such as: gain, phase margin, GBW, ICMR, Slew Rate, Offset, CMRR, output swing etc. also have been analyzed after simulation which is carried out using Cadence Virtuoso Tool. The Op-Amp is designed to display a unity gain frequency of $7.85 \mathrm{MHz}$ and exhibits a gain of $86.23 \mathrm{~dB}$ with a $49^{\circ}$ phase margin. Obtained results also agree with theoretical predictions.
\end{abstract}

Index Terms - CMOS Technology, The operational amplifier, Differential Amplifier 


\title{
FPGA Implementation of Efficient PCA Technique \\ K Duraga Bhavani
}

\author{
${ }^{1}$ Department of ECE, Koneru Lakshmaiah Education Foundation, \\ Green Fields, Vaddeswaram, A.P., India-522502. \\ E-mail: shrutisuman23@gmail.com
}

\begin{abstract}
In the era of VLSI domain, designing a circuit with less power, area, and delay has become challenge for every designer. In this paper we have design and implemented a circuit for 5:2 compressor which is the vital component in CMOS multiplier circuits. The proposed circuit requires less transistor count i.e. 58 transistors. Simulation result shows that proposed 5:2 compressor has substantially expanded power delay performance in contrast to earlier designs.
\end{abstract}

Index Terms - FPGA, Verilog, CORDIC, VLSI. 


\title{
FPGA Implementation of CAD Technique
}

\section{K Duraga Bhavani}

Department of ECE, Koneru Lakshmaiah Education Foundation,

Green Fields, Vaddeswaram, A.P., India-522502.

E-mail:durgabhavani@kluniversity.in.

\begin{abstract}
:
Vedic Mathematics is an ancient Indian algebra in which 16 sutras are used to measure. For excellent performance, most high-speed applications such as cryptography and digital signal processing need powerful and high-speed multipliers. Squaring is a specific case of multiplication. A specialized squaring device can greatly boost the measurement period and significantly reduce the delay. This study discusses the concept of a new square architecture utilizing Vedic-mathematics sutra "Yavadunam." The proposed method uses the amount deficit from the closest base to calculate every operand's circle. The square of a larger number of magnitude is reduced by this method to a smaller multiplication of magnitude and an addition operation.
\end{abstract}

Index Terms -: FPGA, Verilog, CORDIC, CMOS. 


\title{
Smart Particle Swarm Optimization (SPSO) and Its Application in Image Compression
}

\author{
D.P. Tripathi ${ }^{1}$, Mahesh Nayak ${ }^{1}$ \\ ${ }^{1}$ Department of ECE, Koneru Lakshmaiah Education Foundation, \\ Green Fields, Vaddeswaram, A.P., India-522502. \\ E-mail: tripathi@kluniversity.in.
}

\begin{abstract}
:
The qualitative value of an optimization technique depends upon the way it explores and exploits the search region. The proposed Smart PSO (SPSO) is new variant of PSO utilizes the smart behavior of swarm particles in the search space. Unlike the traditional PSO algorithm, During Successive iterations, SPSO does not depends upon the particles initial velocities. It converges the particles towards global optima using cognitive and social knowledge only. This fact has been supported by empirical simulation of ten standard benchmark functions and the comparative results clearly evident that SPSO is better than DIW-PSO, Rand-PSO and QPSO. Further SPSO has been found successfully solves the NP-hard problem vector quantization-based image compression compared to other variants.
\end{abstract}

Index Terms - Decreasing inertia weight PSO (DIW-PSO), Particle swarm optimization (PSO), Quantum particle swarm optimization (QPSO), Random inertia weight PSO (Rand-PSO), Vector quantization (VQ) 


\title{
Chaotic Smart Particle Swarm Optimization (SPSO)
}

\author{
D.P.Tripathi ${ }^{1}$, Mahesh Nayak $^{1}$ \\ ${ }^{1}$ Department of ECE, Koneru Lakshmaiah Education Foundation, \\ Green Fields, Vaddeswaram, A.P., India-522502. \\ E-mail: tripathi@kluniversity.in.
}

\begin{abstract}
:
The qualitative value of an optimization technique depends upon the way it explores and exploits the search region. The proposed Smart PSO (SPSO) is new variant of PSO utilizes the smart behavior of swarm particles in the search space. Unlike the traditional PSO algorithm, During Successive iterations, SPSO does not depends upon the particles initial velocities. It converges the particles towards global optima using cognitive and social knowledge only. Empirical simulation of ten standard benchmark functions and the comparative results clearly evident that SPSO is better than DIW-PSO, Rand-PSO and QPSO. Computation time of SPSO is found less comparable to other taken PSO variants.
\end{abstract}

Index Terms - Decreasing inertia weight PSO (DIW-PSO), Particle swarm optimization (PSO), Quantum particle swarm optimization (QPSO), Random inertia weight PSO (Rand-PSO), Vector quantization $(V Q)$. 


\title{
Wind Driven Algorithm-Based Optimization for Synthesis of
}

\section{Antenna Arrays}

\author{
V.Sahiti \\ Department of ECE, Koneru Lakshmaiah Education Foundation, \\ Green Fields, Vaddeswaram, A.P., India-522502. \\ E-mail: Sahiti.v@kluniversity.in
}

\begin{abstract}
:
The generation of a radiation pattern with application specific constraints requires specific side lobe level and null placement in undesired directions. The wind driven optimization technique is used to achieve the desired SLL and nulls by selecting a suitable objective function. The complex weights of the antenna elements are optimized to produce the desired pattern. It was effectively tested on linear antenna array with less number of elements but can be extended to circular or planar arrays.
\end{abstract}

Index Terms - Arrays, Wind driven optimization, Sidelobe levels, Amplitude, Phase. 


\title{
Planar antenna array synthesis using Cat Swarm optimization
}

\author{
V.Sahiti \\ Department of ECE, Koneru Lakshmaiah Education Foundation, \\ Green Fields, Vaddeswaram, A.P., India-522502. \\ E-mail: Sahiti.v@kluniversity.in
}

\begin{abstract}
:
The natural activity of the cat is observed, and the search space is exploited based on the behavioral characteristics of the cat. The cat's nature to search for food is applied to the field of electromagnetics to obtain the desired shape of the pattern. In this work a pencil beam pattern is synthesized will low SLL. The optimization s achieved by optimizing the positions of elements and the objective function build according to that. The experimental results are performed on a planar array of $20 * 20$ elements and good amount of agreement can be seen after competition with existing works.
\end{abstract}

Index Terms - planar array, Side lobe level, Cat Swarm, positions. 


\title{
Synthesised Copper Nanoparticles incorporated in Polyvinyl Alcohol Nanocomposite for Food Applications
}

\section{Lakshmana Kumar ${ }^{1}$ Dr C Santhosh ${ }^{2}$ and R. S. Ernest Ravindran ${ }^{3}$}

\author{
1,2,3 Department of Electronics and Communication Engineering, \\ Koneru Lakshmaiah Education Foundation, Vaddeswaram, AP, India.
}

\begin{abstract}
:
Copper nanoparticles (CuNPs) are having antimicrobial and anti-viral properties green synthesis provides the cushion for bio-compatibility of CuNPs for drug delivery. Green Synthesis of copper nanoparticle from Capparis zeylanica plant and solution casting it nan composite with Polyethylene Glycol (PEG)/Polyvinyl Alcohol (PVA) that can be utilized for food packaging, drug delivery and cancer therapy, etc., The preparation of copper particles is through aqueous greener synthesis using Capparis zeylanica plant extract as reducing and as well as stabilizing agent. The obtained copper nanoparticles were blended with PEG/PVA biopolymer using solution casting method to obtained nan composite. The synthesized nanoparticles and nan composite were characterized using UV-visible absorption spectroscopy, X-ray diffraction (XRD), Fourier transform infra-red spectroscopy (FTIR), Scanning electron microscopy (SEM), Differential Scanning Calorimentry (DSC), Moisture absorption and Anti-microbial analysis to understand the stability of the resultant product. PEG/PVA + CuNPs nanocomposite shows an excellent characteristic in most aspect that able to fit for bio-medical, engineering and food packaging applications.
\end{abstract}

Index Terms -Greener Synthesis, Copper Nanoparticles, Capparis zeylanica, PEG/PVA. 


\title{
Design of Reversible Binary to Gray and Gray to Binary Converter using QCA
}

\author{
M. Lakshmana Kumar ${ }^{1}$ and R. S. Ernest Ravindran ${ }^{2}$ \\ 1,2 Department of Electronics and Communication Engineering, Koneru Lakshmaiah Education \\ Foundation, Vaddeswaram, AP, India.
}

\begin{abstract}
:
The advancement in technology increases day by day, that the conventional based CMOS VLSI technology reached its peak due to physical limits like power dissipation and size. In order to overcome this, a new alternative method has introduced to recurrently improve the development of digital electronics. Quantum-Dot Cellular Automata (QCA) approach is nanotechnology which is an emerging technology provides an efficient platform to overcome the confines of existing CMOS. In this research investigation a novel design of 4-bit reversible and non-reversible binary to gray (B-G) and gray to binary code (G-B) converter was simulated with the help of QCA tool and the obtained results were found to be efficiently better when compared with that of the previous one.
\end{abstract}

Index Terms - Quantum-dot Cellular Automata (QCA),CMOS, Binary to gray Code Converter, Gray to binary converters. 


\title{
IoT Based Reconfigurable Smart City Architecture
}

\author{
M. Anil Kumar ${ }^{1}$ \\ ${ }^{1}$ Department of Electronics and Communication Engineering, Koneru Lakshmaiah Education \\ Foundation, Vaddeswaram, AP, India.
}

\begin{abstract}
:
Now-a-days Internet of Things is deployed almost in every application regarding smart cities and have been initiated to develop all over the world. Smart Devices are being geared up to ease human life. Due to the growth in Internet of Things technology, smart cities are been developed with this technology to work with the issues of public as well as private. The aim of the article is to enhance a solution to the problems in the smart cities with latest IoT architecture, protocols and services. With the technical support of IoT, using low power Wireless Sensor Networks (WSN) which is connected to transfer the data from M2M applications. In addition to the IoT, intelligent features are integrated with the help of Computer vision makes the technology more flexible. The goal of this article is to engrave the services to challenge the real time environment with low power synthesis technique. Only then, the smart city features are improved and serves the mankind with IoT technology.
\end{abstract}

Index Terms - Quantum-dot Cellular Automata (QCA),CMOS, Binary to gray Code Converter, Gray to binary converters. 


\title{
Brain Tumor Detection from MRI Images Using Optimization Segmentation Techniques
}

\author{
M. Anil Kumar ${ }^{1}$ \\ ${ }^{1}$ Department of Electronics and Communication Engineering, Koneru Lakshmaiah Education \\ Foundation, Vaddeswaram, AP, India.
}

\begin{abstract}
:
The segmentation of medical images is a dynamic and relaxing task because of the distinct features of the images. It is one of the software needed for brain image segmentation to identify the brain tumor and will also be applied to the pre-processing stage to improve detection accuracy. Brain segmentation for magnetic resonance imaging (MRI) is a challenging task given the high-quality Picture quality, anatomical brain structures and tumor complexity. Several researchers have developed several algorithms around tumor segmentation in the field of medical imaging. This work contrasts the effects of optimization with Particle Swam Optimization (PSO) segmentation technique and Darwinian Particle Swam Optimization (DPSO) and Functional Order Darwinian Particle Swam Optimization (FO-DPSO). Using these three chosen methods, the accuracy of the collected data set has been measured and the findings are addressed, the paper from these. We validate these results with the PSO, DPSO and FOPSO, which are commonly used. The method used in the study of medical images. The future work will measure the size of the expected tumor area, which will give the algorithms more accuracy.
\end{abstract}

Index Terms - Greener Synthesis, Copper Nanoparticles, Capparis zeylanica 


\title{
Effective Microstrip Feed Line Length for Ultra Wide Band Responses and Wireless Applications
}

\author{
Mohan K N ${ }^{1}$, K Himaja Reddy ${ }^{1}$, Dr. D Kannadassan ${ }^{2}$, Dr. \\ Jagadish Chandra Mudiganti ${ }^{3}$ \\ 1,2 Department of Electronics and Communication Engineering, Koneru Lakshmaiah Education \\ Foundation, Vaddeswaram, AP, India. \\ ${ }^{2}$ Vellore Institute of Technology, Vellore, Tamilnadu, India ${ }^{3}$ Vellore Institute of Technology, \\ Amaravti, AP, India
}

\begin{abstract}
:
In this article, the effected feed line techniques used instead of DGS (Defected Ground Structure) and CSRR (Coupling Split Ring Resonator) which are commonly used for achieving UWB (Ultra-Wideband Applications) response. The classical antenna designed for $2.4 \mathrm{GHz}$ frequency and feed line used for this patch antenna has designed to $180^{\circ}$ electrical length. The resonance of antenna responses as UWB and it has interesting current distribution through the patch antenna; this paper avoided all aspects of techniques which are proposed to achieve UWB.
\end{abstract}

Index Terms - UWB, CSRR, DGS, Microstrip patch antenna. 


\title{
Audio Denoising by Time-Frequency Block Thresholding
}

\author{
M. Venu Gopala Rao ${ }^{1}$, Ms. Harsha Mane ${ }^{1}$ \\ ${ }^{1}$ Department of ECE, Koneru Lakshmaiah Education Foundation, \\ Green Fields, Vaddeswaram, A.P., India-522502. \\ E-mail: mvgr03@kluniversity.in.
}

\begin{abstract}
:
Audio signals are often contaminated by background environment noise and buzzing or humming noise from audio equipment's. Audio demonising aims at attenuating the noise while retaining the underlying signals. Applications such as music and speech restoration are numerous. Removing noise from audio signals requires a non-diagonal processing of time-frequency coefficients to avoid producing "musical noise". State of the art algorithms perform a parameterized filtering of spectrogram coefficients with empirically fixed parameters. Diagonal time-frequency audio denoising algorithms attenuate the noise by processing each window Fourier or wavelet coefficient independently, with empirical Wiener, power subtraction or thresholding operators. These algorithms create isolated time-frequency structures that are perceived as a "musical noise". The musical noise is strongly attenuated with non-diagonal time-frequency estimators that regularize the estimation by recursively aggregating time-frequency coefficients. We propose a new nondiagonal audio denoising algorithm through adaptive time-frequency block thresholding, which adjusts all parameters adaptively to signal property by minimizing a Stein estimation of the risk. Numerical experiments demonstrate the performance and robustness of this procedure through objective and subjective evaluations.
\end{abstract}

Index Terms - Audio denoising, Block thresholding, Ephraim and Malah, Power spectrum, Power subtraction, Thresholding 


\title{
Automatic Speech Recognition in Meeting Rooms
}

\author{
Dr. M. Venu Gopala Rao ${ }^{1}$, Ms. Harsha Mane ${ }^{1}$ \\ ${ }^{1}$ Department of ECE, Koneru Lakshmaiah Education Foundation, \\ Green Fields, Vaddeswaram, A.P., India-522502. \\ E-mail: mvgr03@kluniversity.in.
}

\begin{abstract}
:
Performance of current speech recognition systems severely degrades in the presence of noise and reverberation. While rather simple and effective noise reduction techniques have been extensively applied, coping with reverberation still remains as one of the toughest problems in speech recognition and signal processing. Single-microphone reverberation algorithms typically result in very low speech recognition performance. Taking advantage of multiple-microphones popularity and theoretical achievements such as the MINT theorem, blind multiple-microphone signal processing techniques are explored and evaluated in several speech recognition test-beds. These include connected digits recognition in meeting rooms and matched and mismatched training-test conditions as well as conversational speech recognition in meetings in mismatched training-test conditions. Multi-channel equalization techniques are shown to be effective under explicit knowledge of either speaker-to-receiver impulse response or order determination, but not in real situations. Blind reverberation techniques based on time-delay estimation, such as delay-and-sum, delay-and-feature-domain-sum and phase-error based filtering are shown to significantly improve recognition accuracy over a single distant micro-phone, while being robust enough for real noisy and reverberant speech. Dereverberation techniques based on linear prediction are introduced and multi-channel correlation shaping is further explored for speech recognition. It is shown to improve recognition accuracy of a single distant microphone only in some situations.
\end{abstract}

Index Terms - Speech recognition, dereverberation, equalization, multichannel, 


\title{
Distributed Peer-to-Peer Data Storage and Synchronizing Websites
}

\author{
Mohan K Na*, T. Maneesh Reddy a, K. Sai Charan a, A. Ganesh a \\ ${ }^{a}$ Department of Electronics and Communication Engineering, KoneruLakshmaiah Education \\ Foundation, Vaddeswaram, Guntur, Andhra Pradesh, India.
}

\begin{abstract}
:
In Peer-to-Peer file distributing systems, content duplication technology is widely accustomed to scale down hot-spots and improve file requesting efficiency. Today's Internet architecture is designed based on the Client-Server model, which establishes lots of challenges. By proposing the protocol of the Peer-to-Peer file duplication application, these challenges will be resolved resourcefully. However, the impact of this protocol will only be in pinnacle when the contributed systems are elevated. With this protocol when implemented in the application of websites then the data - such as website content (back- end, front-end files) can be stored and shared by different individuals whoever visits the website. Additionally, using Bit coin Cryptography and Secure Hash Algorithm (SHA) to encrypt the files and avoid tampering by other peers. For the discovery of peers involved, the Bit Torrent Protocol is applied. This Peer-toPeer file duplication protocol implementation will increase the availability of the website exponentially, concerning the number of peers involved in contributing to the website. In this paper we will execute a Decentralized Peer-to-Peer based application for file synchronizing and storing, using the Bit coin Cryptography and Bit Torrent Protocols.
\end{abstract}

Index Terms -BitTorrent protocol; Distributed storage;File synchronization; Peer-to-Peer. 


\title{
Design and Analysis of Reconfigurable antenna for On-Body Applications
}

\author{
M Siva Kumar \\ Department of Electronics and Communication Engineering, KoneruLakshmaiah Education \\ Foundation, Vaddeswaram, Guntur, Andhra Pradesh, India.
}

\begin{abstract}
:
Health care industry widen its application specific network connection with embedding several sensors into its scope with wireless body area network and personal area networks. Several products came into existence with the advancements in the integrated industry of health care instrumentation, wireless connectivity, and remote monitoring systems with IOT interfacing. Compact and accurate devices with reliability playing a vital role in the health care industry with advent of advanced communication interfacing. The wireless communication devices providing sustainable services with the integration of modern antenna for various applications. The advanced antenna system is standing at forefront to all wireless communication devices with their reliable and trustworthy performance. In the health care industry, majority of the communication modules are turning towards remote controlled devices with IOT interfacing. The modern antennas require the qualities like compactness, low weight, conformability/flexibility and multi operating band capability to utilise them in health care communication modules. The sensing characteristics in the microwave region with these modern antennas are enabling desired radiation mechanism with reconfigurable designs.In this work the design and development of RF-MEMS based reconfigurable antenna for wireless body are network applications has been proposed. The designed antenna covering the applications frequencies of 2.4-2.5 GHz (Wi-Fi, Bluetooth \& LTE) and 5.8-5.9 GHz(WLAN \&ISM) applications in the range of UWB.
\end{abstract}

Index Terms - IOT, RF, WLAN, UWB, Bluetooth. 


\title{
Circularly Polarized Ring Antenna with Spokes for
}

\section{ISM Band Applications}

\author{
M Siva Kumar \\ Department of Electronics and Communication Engineering, KoneruLakshmaiah Education \\ Foundation, Vaddeswaram, Guntur, Andhra Pradesh, India.
}

\begin{abstract}
:
The article presents dual band antenna with compact size for ISM band applications. The proposed antenna operates at two frequencies ranging from $2.1-3.1 \mathrm{GHz}$ and 4.4-7.7 GHz with resonating frequencies at $2.45 \mathrm{GHz}$ and $5.8 \mathrm{GHz}$. The antenna is fed by CPW line, which consists of 24 spokes and asymmetric ground structure. The antenna provides the impedance bandwidth of $38.4 \%$ and $49 \%$ at two operating bands. The antenna provides circular polarisation with $3 \mathrm{~dB}$ axial ratio bandwidth of $150 \mathrm{MHz}(2.33-2.48 \mathrm{GHz})$ and $1600 \mathrm{MHz}(5.14-6.74 \mathrm{GHz})$. The proposed antenna is fabricated on RT Duriod 5880 with dimensions of $40 \times 28 \times 0.4 \mathrm{~mm}^{3}$. As the antenna is designed for wearable application it is placed on human phantom at different bending angles and analyzed the performance. The SAR values observed at two operating frequencies is $1.09 \mathrm{~W} / \mathrm{Kg}$ and $1.47 \mathrm{~W} / \mathrm{Kg}$ respectively. To analyse the far-field patterns Ansys Savant simulation tool is used.
\end{abstract}

Index Terms -SAR, ISM, Frequency, Patterns. 


\title{
International Journal for Innovative
} engineering and Management Research

A Peer Revieved Open Access International Journal

\section{A Modified Hybrid Planar Antenna for Cognitive Radio Application}

\section{Arjuna Muduli*, Rabindra Kishore Mishra}

*Department of ECE, Koneru Lakshmaiah Education Foundation, Vaddeswarm, Vijayawada, Andhra Pradesh, India

Department of Electronic Science, Berhampur University, Berhampur, Odisha, India

Email id: mr.arjuna.muduli@ieee.org

\begin{abstract}
:
This article approaches an integrated planar antenna configuration for CR communication. There is a rectangular microstrip antenna for narrowband operation and a monopole antenna along with CPW feed concept for wideband operation is printed on the single side of substrate. This configuration reduces coupling effect. The narrow band microstrip antenna helps the CR for communication and the sensing of spectrum is done by the ultrawideband monopole structure.
\end{abstract}

Keywords: Microstrip antenna, MSA, UWB, CR 
An Ultra-Wide Band Octagonal Antenna with Reconfigurable Narrowband Antenna for Cognitive Radio Applications

\title{
Arjuna Muduli, Buddi Bhanu Shankar, Dasari Anantha Sai Kumar, Garlapati Venkata Kowndinya, Deepak Kumar Nayak
}

Department of ECE, Koneru Lakshmaiah Education Foundation, Vaddeswarm, Vijayawada, Andhra Pradesh, India

Email id: arjuna@kluniversity.in

\begin{abstract}
:
The idea of this paper is to design a dual port ultra-wide band antenna integrated with reconfigurable narrow band antenna for cognitive radio applications. The narrow band antenna is a microstrip patch antenna attached at the back side of the ultra-wide band octagonal antenna. The ultra-wide band senses over the frequency range of $3.91-11.29 \mathrm{GHz}$. The Narrowband reconfigurable antenna is seen to operate at $6.39 \mathrm{GHz}, 6.90 \mathrm{GHz}, 7.81 \mathrm{GHz}, 8.94 \mathrm{GHz}$. Isolation between both the ports is kept less than $-15 \mathrm{~dB}$ over entire operating band. Reconfiguration is done with the help of pin diode. The antenna switched from ultra-wide band to narrow band by switching 'ON' the pin diode.
\end{abstract}

Keywords: Cognitive radio, ultra-wide band, narrowband, return loss. 
Al based programmed imperfection location using Thermal wave imaging

\author{
Muzammil Parvez M \\ Department of ECE, Koneru Lakshmaiah Education Foundation, Vaddeswaram, A.P., India- \\ 522502. \\ Email: parvez@kluniversity.in
}

\begin{abstract}
:
Discovery of subsurface non consistency is pivotal in choosing the quality of articles for different modern applications. Non fixed warm wave imaging is developing as a dependable subjective evaluation methodology to recognize irregularities in a wide scope of materials. This paper proposes a directed AI based characterization methodology to distinguish the subsurface deformities utilizing quadratic recurrence tweaked warm wave imaging and experimentation has been persisted glass fiber Reinforced polymer material (GFRP) with 10 Teflon patches having various profundities and sizes and Carbon fiber Reinforced polymer (CFRP) with 25 base openings having various sizes and profundities. In this paper three well realized regulated AI methods, Decision tree (DT), Support vector machine (SVM) and k-Nearest neighbor (KNN) classifiers are utilized for imperfection location. Location ability and unwavering quality of deformity discovery have been surveyed utilizing sign to clamor proportion and likelihood of location individually.
\end{abstract}

Keywords: infrared thermograph, fibre reinforced polymers, classifiers and quadratic frequency modulated thermal wave imaging. 


\title{
Anomalies detection in quadratic frequency modulated thermal wave imaging using Fuzzy C-means clustering
}

\section{Muzammil Parvez M}

Department of ECE, Koneru Lakshmaiah Education Foundation, Vaddeswaram, A.P., India522502.

\section{Email:parvez@kluniversity.in}

\begin{abstract}
Deformity portrayal from its non-deficient partner from raw thermal response plays a vital role in Quadratic frequency modulated thermal wave imaging(QFMTWI). The quality of the bone lessens due to the skeletal jumble as the age of the individual develops, Early conclusion comparing to illness is important to give great bone quality. By distinguishing bone thickness varieties the illness can be overseen adequately. A non-fixed warm wave imaging strategy, Quadratic frequency modulated thermal wave imaging (QFMTWI) is utilized to portray severity of the human bone, just as experimentation additionally carried on Carbon fiber reinforced polymers (CFRP) test and are reached out to solo AI calculations like k-implies grouping and fuzzy c-implies bunching calculations. If there should arise an occurrence of an spectator with less mastery, an ideal solo grouping approach is important to satisfy this necessity. In present article, we applied k-implies and Fuzzy-c-implies based solo bunching methods for subsurface imperfection recognition in QFMTWI. The pertinence of these calculations is tried on a mathematical mimicked biomedical bone example having different thickness varieties and an exploratory Carbon fiber reinforced polymers (CFRP) test with level base openings of various profundities with same size. Sign to Noise Ratio (SNR) is taken as execution merit and on correlation, we finish up Fuzzy c-implies gives better recognition and portrayal of surrenders contrasted with K-means grouping for QFMTWI
\end{abstract}

Keywords: Infrared thermograph, clustering and Quadratic frequency modulated thermal wave imaging. 

Engineering and Management Research

A Peer Revieved Open Access International Journal

Design Analysis and Fabrication of FinFET using 3nm Technology

\title{
${ }^{1}$ B. Balaji, ${ }^{2}$ E. Radha
}

Department of ECE, Koneru Lakshmaiah Education Foundation, Vaddeswaram, A.P., India 522502 .

Assoc. Professor, BRIL, Hyderabad, E-mail ID: balaji@kluniversity.in, TS.radhaareddy@gmail.com

\begin{abstract}
The performance analysis of FinFET design is newest technology compared to conventional bulk FinFET and designed nanowire gate-all-around (GAA) FET using Three-dimensional simulator Silvaco TCAD tool and had done for both n-channel and p-channel GAA FET transistors. The results of this design (FinFET) provide for improved sub threshold swing, sub threshold voltage, on current, off current and DIBL on electrostatic energy relative to FinFET, but less gate capacitance relative to the GAA FET. So FinFET technology offers as an industry enhances for continues transistor scaling with good performance improvement for low-power system-on-chip and variousapplications.
\end{abstract}

Index Terms: Fin FET, gate-all-around (GAA) FET, system-on- chip (SoC), nano wires 
Investigation on Cluster based Application Mapping Approach for Power Optimization in NoC

\author{
Aravindhan Alagarsamy ${ }^{1}$, Sundarakannan Mahilmaran ${ }^{2}$ \\ ${ }^{1}$ Department of ECE, Koneru Lakshmaiah Education Foundation, Vaddeswaram, A.P., India- \\ 522502. \\ ${ }^{2}$ Department of Mathematics, Sri Sivasubramaniya Nadar College of Engineering, Kalavakkam, \\ Chennai - 603110, Tamilnadu, India. \\ drarvindhan@kluniversity.in, sundarakannanm@ssn.edu.in
}

\begin{abstract}
Network-on-Chips (NoCs) are one of the emerging research areas that has several research problems that need to be addressed. Reducing the power consumption itself is one important problem for NoC based systems. Various methods are proposed till now to reduce the power consumption in NoC. Unless, for getting better results, cluster-based mapping method is proposed. In this paper we propose a new cluster-based mapping method is proposed with FM algorithm for reducing power consumption. On the other hand, an optimization technique called Tabu search is used for reducing power consumption. The performance and efficiency of proposed methodology was verified with the experiments conducted in different benchmarks in NoC. Experimental results show that Tabu search is unsuitable for power calculation.
\end{abstract}

Index Terms: Network-on-Chips (NoC), Cluster Mapping, Tabu search 
Modified Bat Algorithm for Cluster based Application Mapping Method in 2D NoC

\author{
Aravindhan Alagarsamy ${ }^{1}$, Lakshminaraynan Gopalakrishnan ${ }^{2}$ \\ ${ }^{1}$ Department of ECE, Koneru Lakshmaiah Education Foundation, Vaddeswaram, A.P., India- \\ 522502 \\ ${ }^{2}$ Department of Electronics and Communication Engineering, National Institute of Technology, \\ Tiruchirappalli - 620015, Tamilnadu, India. \\ drarvindhan@kluniversity.in, laksh@nitt.edu
}

\begin{abstract}
Due to the continuous scaling in the design of large-scale integrated circuits (VLSI), the networkon-chip (NoC) has become one of the growing research areas and has to solve many research problems. In this study, we propose a modified BAT algorithm (MBA) for successful cluster-based bandwidth and power awareness mapping system for 2D NoC. The proposed method is verified by the number of experiments conducted in the NoC with different types of standard definitions.
\end{abstract}

Index Terms: Network-on-Chips (NoC), Modified bat algorithm (MBA), Power aware mapping 


\title{
Detection of Objects for Autonomous Cars Using Lane Detection Method
}

\author{
Dr. P.P. Priya ${ }^{1}$, K. Pavan Kumar ${ }^{2}$, MD. Akram Hussain ${ }^{2}$, S.CH. S.S. \\ Teja $^{2}$, K. Sampath ${ }^{2}$ \\ Department of ECE, Koneru Lakshmaiah Education Foundation, Vaddeswaram, A.P., \\ India-522502.
}

\begin{abstract}
This paper presents two algorithms, one to detect, track objects and the other for lane line detection. This detection and tracking of objects are done by using a Tensor flow object detection API. And a simple computer vision technique is obtained for Lane line detection. The objects Location which are identified that is forwarded to the algorithm of object detection and tracking. The object detection tracking system algorithm is used for obtain by using CVV. The proposed approach can able to detect objects in different illumination and occlusion. It accuracy of achieved is $90.88 \%$ on self-generated image sequences.

Key-Words: Detection, Algorithm, Detection
\end{abstract}




\title{
Design of Birds Detector and Repellent Using Frequency Based Repeller By Machine Learning Algorithm
}

\section{Dr P. P. Priya ${ }^{1}$, G Pavankalyan², P Siva Swapnic guptha ${ }^{3}$, Sk Juber Ahamed ${ }^{4}$ \\ Department of ECE, Koneru Lakshmaiah Education Foundation, Vaddeswaram, A.P., India-522502.}

\begin{abstract}
Among entire globe many Countries people work as farmers to survive their lives. Plants are interconnected to farming and pests which are related to plants, where there are bulk of plants then they lead to farming there must be effect causing animals called pests. Pests are not only animals they may be birds too that damage the plants. One of the examples of pests are birds and monkeys that eat plants and its products and cause harm to farmers. In This research technique we has developed and designed a prototype of birds detector and repellent of birds based on frequency. This designed model has a Bird detection and repellent system which detects the bird based on its given python code as well as with the help of some machine learning algorithms and then proceeds further step to a repellent which produces a certain frequency with respective the birds by classification the birds based on their resonating frequencies, so the bird will be disturbed through the repellent frequency and goes away from it. This designed model consists of python open cv software as a detector to detect movement from birds and further classifies the type of bird and for that type it gives signals to further step to fire certain signal frequency to repeller. The repeller is designed using LC oscillator type Colpitts with Piezo Ultrasonic sensor as repeller which produces. The obtained results of the experiment are mainly focused on Birds with bird detection system, which is capable of working with a detection distance between $2 \mathrm{~m}-500 \mathrm{~m}$ by using sensors to detect bird, and by modifying this model in aspect of sensor we used machine learning algorithm of python open $\mathrm{cv}$ it increases the range of detecting the bird from $500 \mathrm{~cm}$ to 500 meters and can continue for further process to repel the birds at a frequency signal of about $60 \mathrm{kHz}$.
\end{abstract}

\section{KEY-WORDS: Oscillator, Detection, Algorithm, Detection}




\title{
A novel approach in optimal parameters selection for high performance and ultra-low power $\Sigma \Delta$ Modulator
}

\author{
S. Vamsee Krishna ${ }^{1}$ M. Lakshmana Kumar ${ }^{2}$ and R. S. Ernest Ravindran ${ }^{3}$ \\ ${ }^{1,2,3}$ Department of Electronics and Communication Engineering, Koneru Lakshmaiah Education \\ Foundation, Vaddeswaram, AP, India.
}

\begin{abstract}
A linear model analysis is made for second to fourth order cascaded, single loop and hybrid sigma delta modulator architectures with a novel technique for deriving choice of parameters. Parameters obtained in this paper by considering the trade-offs between architectural to physical level implementation in top-down design approach, which guides efficient practical implementation of sigma delta modulator. With the proposed choice of parameters, the performance metrics of sigma delta modulators are verified in behavioural modelling and comparison of selected architectures are given for optimum selection of circuit topology and modulator parameters in design of SigmaDelta analog to digital converters.
\end{abstract}

Index terms: Sigma delta modulator, top-down design, behavioral modeling. 


\title{
Systematic design and analysis of 3rd order single loop $\Sigma \Delta$ Modulator for ECG signal processing
}

\section{S. Vamsee Krishna ${ }^{1}$ M. Lakshmana Kumar ${ }^{2}$ and R. S. Ernest Ravindran ${ }^{3}$}

1,2,3 Department of Electronics and Communication Engineering, Koneru Lakshmaiah Education Foundation, Vaddeswaram, AP, India.

\begin{abstract}
This paper presents system level modeling of sigma delta modulator including circuit level non idealities in MATLAB/Simulink environment for low frequency applications. An overview of sigma-delta modulation technique in analog-to-digital conversion and implementation of $\sum \Delta$ analog-to- digital converters using different technology processes, architectural and circuit level methods explored. A third order single loop sigma delta modulator is designed and verified with careful selection of integrator weights to meet stable desired performance. The proposed designed achieved SNDR of $101.75 \mathrm{~dB}$ and 17-bit resolution. The simulation performed at behavioral level using Simulink based sigma delta simulator, the results obtained are accurate, by considering non ideal effects. The results obtained for the proposed cascaded sigma delta modulator compared with existing single loop and cascaded architectures and proved as efficient.
\end{abstract}

Index terms: analog-to-digital conversion, $\Sigma \Delta$ modulator, CMOS. 


\title{
An Optimal Hybrid Solution to Local and Global Facial Recognition Through Machine Learning
}

\author{
K. Raju', B. Chinna rao ${ }^{2}$, N.L. Pratap ${ }^{3}$ \\ ${ }^{1}$ Department of ECE, Narasaraopet Engineering College, Narasaraopet, Guntur, AP, India. \\ ${ }^{2}$ Department of ECE, NS Raju Institute of Technology, Visakhapatnam, India. \\ ${ }^{3}$ Nalajala Lakshman Pratap, Asst Prof, Department of Electronics and Communication \\ Engineering, Koneru Lakshmaiah Education Foundation, Vaddeswaram, AP, India. \\ raju.kolluri@gmail.com, lakshmanpratap@kluniversity.in, profbcrao@gmail.com
}

\begin{abstract}
Face recognition need is fine assured as enormous industrial relevance to use them toimplement one or another objective. As facial recognition has beating advantages over other industrial applications and human eyes can easily evaluate performance, improved algorithms and smaller computing costs are continuously improving this methodology. This research takes the conventional algorithms of recognition in the first stage and use hybrid approaches to counter their limitations. The research starts with basic computation of global face features using Principal Component Analysis, Discrete Wavelet Transform and Independent Component Analysis, with some standard NN classifiers and Support Vector Machine. It has observed that as learning rate are high in machine learning then accuracy of system goes high but increases the area and cost overhead. To overcome that training limitation, a Fusion based methods has been proposed in further work, based on Harris corner, Speed Up Robust Features and DWT+PCA system model where only $10 \%$ training sample has been taken on Essex database and $99.45 \%$ accuracy is achieved. To overcome limitations further an efficient Hybrid method proposed which elaborates the local features of face. Further these features trained with Neural Network classifier to obtained better accuracy nearly $99.40 \%$ with single image training from each class.
\end{abstract}

Index Terms - dwt, pca, ica, lbp, hog, glcm, harris corner. 


\title{
Data Security in Cloud Computing Using Three-Factor
}

\section{Authentication}

\author{
Sunanda Nalajala ${ }^{1}$, B. Moukthika ${ }^{1}$, M. Kaivalya ${ }^{1}$, N.L. Pratap ${ }^{2}$ \\ ${ }^{1}$ Department of CSE, Koneru Lakshmaiah Education Foundation, \\ Green Fields, Vaddeswaram, A.P., India-522502. \\ ${ }^{2}$ Department of Electronics and Communication Engineering, Koneru Lakshmaiah Education \\ Foundation, Green Fields, Vaddeswaram, A.P., India-522502 \\ E-mail: lakshmanpratap@kluniversity.in, sunanda@kluniversity.in.
}

\begin{abstract}
Cloud storage is tremendously increasing its services, huge range of storage and communication of massive data over network. This practically has an adverse effect on the way of upholding this data when it especially comes to the privacy of the user-security. We first view you with a system that is vulnerable to this authentication protocol with its misuse of biometrics and incorrect password generates no user to lose the mobile device. We even went along with this scheme and gave out a major issue to overcome with impersonation attack. However, this scheme makes the way easy to attack for offline password guessing attack. We included a three-factor authentication which carries the smart card into card reader, gets the password and identity and conveys the user details' requesting time. We then came up with a scheme to overcome these security flaws of this prescribed authentication scheme combining passwords, mobile devices, and biometrics. The proposed system is robust three-factor authentication with the help of password, biometrics and mobile device which provides reliable security strength to the user's data and makes counterattack to existing attack, giving with more benefits compared to the previous scheme. This scheme provides with most enhanced security functionalities.
\end{abstract}

Index Terms -Three-factor authentication Counter attacks Privacy Biometrics Smart cards 


\title{
Error Detectable Carry Save Adder
}

\section{S. Rooban ${ }^{1}$, V. Easwar ${ }^{2}$}

\author{
Associate Professor ${ }^{1}$, Student ${ }^{2}$, Department of ECE, Koneru Lakshmaiah Education Foundation, \\ Vaddeswaram, A.P., India-522502. \\ E-mail ID: rooban@kluniversity.in'1, $\underline{\text { hanumanthakk92971@gmail.com² }}$
}

\begin{abstract}
A error detectable adder is proposed. Carry save adder is taken as the reference to implement the proposed work. Predicted parity for the sum and carry is compared with the actual sum and carry of the carry save adder. That differences in the comparison shows the erroneous output. The reliable system requires the error detectability during the circuit is in active mode and the prediction of fault before its occurrences. This proposed carry save adder detects the error and contributes $2 \%$ improvement in the overall reliability of the circuits.
\end{abstract}

Index Terms: Concurrent error detection, Carry select adder, Design for testability. 


\title{
Path Delay Fault coverage improvement in LFSR
}

\author{
S. Rooban', V. Easwar ${ }^{2}$
}

\author{
Associate Professor ${ }^{1}$, Student ${ }^{2}$, Department of ECE, Koneru Lakshmaiah Education Foundation, \\ Vaddeswaram, A.P., India-522502. \\ E-mail ID: rooban@kluniversity.in ${ }^{1}, \underline{\text { hanumanthakk92971@gmail.com }}{ }^{2}$
}

\begin{abstract}
Test data compression logic is based on both the compression strategy and the decompression strategy applied. The number of compression test applied to both compression base and the decompression can be reduced my adopting various methods. Quality of the test for path delay coverage can be improved by keeping the same test set and applying many times by changing sequence using effective algorithm. An algorithm to apply the compression phase is proposed so that the LFSR is used effectively. The effective usage of the LFSR increases the quality and improves the path delay coverage capability of the VLSI circuits.
\end{abstract}

Index Terms: Linear Feedback Shift Register (LFSR), Path delay faults (PDFs), Test Data Compression, Test Generation 


\title{
Facial Detection and Recognition System on Raspberry pi with Enhanced Security
}

\section{Sri Sai Mahesh, T. Maneesh Reddy, A. Sai Yaswanth, C Joshitha, S. Sudarshan Reddy}

\author{
Department of ECE, Koneru Lakshmaiah Education Foundation, \\ Vaddeswaram, A.P., India-522502. \\ E-mail ID: joshi1509@kluniversity.in
}

\begin{abstract}
In the present day in our daily life, we all depend on the Internet for web browsing, e-mail, and peer-to-peer services to fulfill our needs. The word Internet means Internetworking of things but IoT (Internet of Things) means a physical object that had a feature of Internet protocol address and that will make the communication between the object and other internet-enabled devices. Here to provide security between the communicating devices without any delay is the main important factor. To provide more security to the existing Face recognition and detection system in homes and banks we propose a new system that will extend the current system. In this paper, we have briefly described the requirement to make such a system and Face recognition Algorithm for Authentication purposes and sending the data using Telegram bot.
\end{abstract}

Index Terms: IoT, Raspberry Pi 3, OpenCV Face recognition, Telegram bot 


\title{
Fabric Substrate Material Based Multiband Spike Antenna for Wearable Applications
}

\author{
P. Syam Sundar, B.T.P. Madhav, D. Sri Harsha, P. Manasa, G. Manikanta \\ and K. Brahmaiah
}

Department of ECE, Koneru Lakshmaiah Education Foundation, Vaddeswaram, AP, India 522502.

E-mail ID: syamsundarp@kluniversity.in

\begin{abstract}
A different multiband spike antenna with fabric material as substrate for wearable applications is presented in this study. Five fabric materials, including Silk, Aravind Cotton, Lenin, Raw Silk and Terry-Cotton are been used in this study. The performance characteristics, like gain, impedance bandwidth, efficiency and radiation characteristics are simulated and measured. The performance characteristics of the wearable antennas in the $\mathrm{S}$ and $\mathrm{L}$ band are observed and presented in this study. Generally, all the evaluated antennas are giving estimated outputs and meeting the requirements of practical aspects.
\end{abstract}

Keywords: Fabric substrate material, impedance bandwidth, multiband spike antenna, performance characteristics, wearable applications 


\title{
PENTAGON SHAPED MICROSTRIP ANTENNA FOR WIRELESS IOT APPLICATIONS
}

\section{P. Syam Sundar ${ }^{1}$, Sarat K Kotamraju ${ }^{2}$, Sri Kavya K $\mathrm{Ch}^{2}$, BTP Madhav², Y Srikanth $^{3}$, Narendra Babu A ${ }^{4}$}

Department of ECE, Koneru Lakshmaiah Education Foundation, Vaddeswaram, AP, India 522502.

Department of ECE, S R Engineering College, Warangal-506371, Telangana India Department of ECE, LBRCE, Andhra Pradesh, India

\begin{abstract}
A monopole pentagon shaped microstrip patch antenna for IoT applications is presented in this article. Different iterations are performed to in the design process of the proposed antenna. The proposed antenna has 40mm X 40mm X 1.6mm dimensions and is designed using FR4 substrate. The proposed antenna has defected ground structure (DGS) in its design. The proposed antenna has triple resonance bands which covers from 4.10-5.13GHz, 5.21-5.81GHz (IoT band) and 6.24$7.87 \mathrm{GHz}$ respectively. At the IoT resonance band the proposed antenna has an average gain of $2.11 \mathrm{dBi}$ and it varies from 1.60-2.66dBi. The proposed antenna has $11.11 \%$ impedance bandwidth at the second resonance band. The proposed antenna has $86.52 \%$ radiation efficiency, which varies from 78.5-90\% in the specified IoT band. The antenna is designed and simulated using ANSYS Electronic Desktop-2018. The proposed antenna shows good gain and radiation efficiency, etc., which are important in IoT wireless applications.
\end{abstract}

Key words: IoT, DGS, impedance bandwidth, efficiency, ANSYS Electronic Desktop. 


\title{
TRANSMISSION LINE EFFECTS AND CROSS TALK EFFETS ON INTERCONNECTS IN DEEP SUBMICRON VLSI
}

\author{
S. A. Sivasankari ${ }^{1}$, Dr. D. Dhanasekaran ${ }^{2}$ \\ Department of ECE, Koneru Lakshmaiah Education Foundation, Vaddeswaram, A.P., India- \\ 522502.
}

\begin{abstract}
This paper investigates the cross-talk effects in interconnects. Here, it is described how cross talk effects occurred. In section 2 , the discussion is on how the delay model can be formulated, presented and designed the adder model also. This work which presents a new and computational efficient performance optimization technique for distributed RLC interconnects. In Section 3 it is discussed the model and strategy and discussed the wire model as well as the geometry also. In substrate effect model the concept explained how the interference gets released. In section 4 analysis and effects are considered and discussed. In section 5 the different structure techniques are explained. The results which are discussed at the end of the work. This work concluded how delay and power are reduced in Deep submicron VLSI interconnects.
\end{abstract}

Keywords: Cross talk, Interconnects, Delay Model, Shielding, DSM interconnects,Transistor order, Cross talk noise effects, Telegraphers equation, EP approach. 


\title{
Epilepsy Seizure Alert System using IoT
}

\section{S. Sweet line Shamini ${ }^{1}$, R. Ohmsakthi Vel ${ }^{2}$, S. A. Sivasankari ${ }^{3}$, J. K. Periasamy $^{4}$, G. Ramprabu ${ }^{5}$}

Department of ECE, Koneru Lakshmaiah Education Foundation, Vaddeswaram, A.P., India522502.

\begin{abstract}
The main objective is to detect the epilepsy seizure in advance, by implementing hardware devices for detection. We are using arduino to monitor the epileptic disease in human and prevent it at its early stage of the disease. In our method we are proposing ECG sensors to monitor the human cardiac signals and update it in the LCD monitor using IOT. We have analyzed the predefined data set of diseased cardiac signal using arduino. If the human cardiac signal is same as the data set signal and then it provides the information that the human is epileptic and the patient's database is sent through IOT to authorized doctor. We are also using a heartbeat sensor to monitor the pulse rate of the human. Every change in the patient will be monitored updated through IOT.
\end{abstract}

Key words: ECG Sensors, Heart Beat Sensors, Arduino, Internet of Things, LED display 
Synthesised Copper Nanoparticles incorporated in Polyvinyl Alcohol

\title{
Nanocomposite for Food Applications
}

\author{
M. Lakshmana Kumar ${ }^{1}$ Dr C Santhosh ${ }^{2}$ and R. S. Ernest Ravindran ${ }^{3}$ \\ ${ }^{1,2,3}$ Department of ECE, Koneru Lakshmaiah Education Foundation, Vaddeswaram, A.P., India- \\ 522502. \\ E-mail ID: lakshmana.muna@gmail.com
}

\begin{abstract}
Copper nanoparticles (CuNPs) are having antimicrobial and anti-viral properties green synthesis provides the cushion for bio-compatibility of CuNPs for drug delivery. Green Synthesis of copper nanoparticle from Capparis zeylanica plant and solution casting it nanocomposite with Polyethylene Glycol (PEG)/Polyvinyl Alcohol (PVA) that can be utilized for food packaging, drug delivery and cancer therapy, etc., The preparation of copper particles is through aqueous greener synthesis using Capparis zeylanica plant extract as reducing and as well as stabilizing agent. The obtained copper nanoparticles were blended with PEG/PVA biopolymer using solution casting method to obtained nanocomposite. The synthesized nanoparticles and nanocomposite were characterized using UV-visible absorption spectroscopy, X-ray diffraction (XRD), Fourier transform infra-red spectroscopy (FTIR), Scanning electron microscopy (SEM), Differential Scanning Calorimentry (DSC), Moisture absorption and Anti-microbial analysis to understand the stability of the resultant product. PEG/PVA + CuNPs nanocomposite shows an excellent characteristic in most aspect that able to fit for bio-medical, engineering and food packaging applications.
\end{abstract}

Keywords: Greener Synthesis, Copper Nanoparticles, Capparis zeylanica, PEG/ PVA, XRD, FTIR, SEM. 
Design of Reversible Binary to Gray and Gray to Binary Converter

\title{
using QCA
}

\author{
M. Lakshmana Kumar ${ }^{1}$ and R. S. Ernest Ravindran ${ }^{2}$ \\ ${ }^{1,2}$ Department of ECE, Koneru Lakshmaiah Education Foundation, Vaddeswaram, A.P., India- \\ 522502. \\ E-mail ID: lakshmana.muna@gmail.com
}

\begin{abstract}
The advancement in technology increases day by day, that the conventional based CMOS VLSI technology reached its peak due to physical limits like power dissipation and size. In order to overcome this, a new alternative method has introduced to recurrently improve the development of digital electronics. Quantum-Dot Cellular Automata (QCA) approach is nanotechnology which is an emerging technology provides an efficient platform to overcome the confines of existing CMOS. In this research investigation a novel design of 4-bit reversible and non-reversible binary to gray (B-G) and gray to binary code $(\mathrm{G}-\mathrm{B})$ converter was simulated with the help of QCA tool and the obtained results were found to be efficiently better when compared with that of the previous one.
\end{abstract}

Key words: Quantum-dot Cellular Automata (QCA), CMOS, Binary to gray Code Converter, Gray to binary converters. 


\title{
Reduction of Peak to Average Power Ratio in OFDM System by Using Selected Mapping Technique
}

\author{
T Penchala Naidu ${ }^{1}$, Dr. M Venkata Narayana ${ }^{2}$ \\ ${ }^{1}$ Assistant Professor, ${ }^{2}$ Professor, Department of ECE, Koneru Lakshmaiah Education \\ Foundation, Vaddeswaram, A.P., India-522502, penchalanaidu@kluniversity.in
}

\begin{abstract}
The primary disadvantages of OFDM is high PAPER. The chose planning procedure is one of the most famous PAPR decrease plans for the OFDM framework. The current plan, which is utilized to decrease PAPR, is separating. In Filtering plan, there might be a chance of sign misfortune. So the separating strategy is certifiably not a best procedure to lessen PAPR in OFDM framework. Another procedure used to decrease PAPR is cutting. In the cut-out cycle, the first sign might be lost. The computational multifaceted nature of both separating and cutting is less, yet correspondence framework execution will endure with the utilization of these strategies because of the loss of wanted sign. Presently the proposed procedure is SLM. In the SLM procedure, the regulated data is duplicated with various stage arrangements lastly, we select the increased stage information with negligible PAPR. The OFDM framework with SLM is to some degree complex however the estimation of PAPR will be decreased. The other huge disadvantage of the OFDM framework is ISI. The ISI is there due to the multipath impact. To diminish the ISI content, we are utilizing the RAKE recipient. The beneficiary is planned so that it gets all the multipath signals, at that point does the time scaling to arrange all the multipath signals, lastly midpoints all the timescaled multipath signals. The proposed method is SLM. The computational unpredictability of translating OFDM with SLM is substantially less than that of OFDM framework. The recreation results show the PAPR decrease execution.
\end{abstract}

Index Terms: OFDM-Orthogonal Frequency Division Multiplexing, PAPR-Peak to Average Power Ratio, ISI-Inter Symbol Interference, SLM-Selected Mapping. 


\title{
Evaluation of Adverse Effects of Fog on FSO links
}

\author{
A. Sree Madhuri ${ }^{1}$, Govardhani.Immadi ${ }^{2}$, M. Venkata Narayana ${ }^{3}$ \\ ${ }^{1,2,3}$ Department of ECE, Koneru Lakshmaiah Education Foundation, \\ Green Fields, Vaddeswaram, A.P., India-522502. \\ E-mail: asreemadhuri@kluniversity.in.
}

\begin{abstract}
The adverse effects of fog on FSO links is estimated with the help of simulation and experimental setup. The simulation and experimental results have followed a similar pattern and the pattern was like as the attenuation was increasing the signal strength started to deteriorate. This clearly indicates the need for a prior study of atmospheric effects on FSO link before being implemented in the real scenario. As FSO links were already in use for intersatellite communication purpose they will also be utilized for the earth station to satellite links in the near future which increases the necessity to study the link behavior
\end{abstract}

Index Terms - Fog, link, communication, experimental setup, attenuation. 


\title{
Understanding the effects of various modulation techniques on FSO links
}

\author{
A. Sree Madhuri ${ }^{1}$, Govardhani.Immadi ${ }^{2}$, M. Venkata Narayana ${ }^{3}$ \\ 1,2,3 Department of ECE, Koneru Lakshmaiah Education Foundation, \\ Green Fields, Vaddeswaram, A.P., India-522502. \\ E-mail: asreemadhuri@kluniversity.in.
}

\begin{abstract}
FSO utilizes the light propagating in the free space to transmit the data wirelessly for both computer networking and communication domains. In optical communication, we use optical fiber cable as a medium but in the free space optical communication, we use the free space as the medium to propagate the light signal. Here free space means air, or outer space. Free Space Optics is a line of sight communication. Here the link is in the range of 500 meters, 1500 meters and 2000 meters, a CW laser of $10 \mathrm{db}$ and at wavelength of $1550 \mathrm{~nm}$ is constructed under frail disturbance like climatic initiated attenuation, scattering is assessed through different modulation techniques, to be specific ASK, PSK, FSK, Dual Drive MZ.
\end{abstract}

Index Terms - Scattering Fog, link, communication, experimental setup, attenuation. 


\title{
A Novel Low-Power 5th order Analog to Digital Converter for Biomedical Applications
}

\author{
Aditya $M^{1}$, I. Veer Raghava Rao ${ }^{2}$, B. Balaji ${ }^{3}$, John Philip $B^{4}$
}

Department of ECE, Koneru Lakshmaiah Education Foundation, Vaddeswaram, A.P., India522502

aditya@kluniversity.in, raghva.inumula@kluniversity.in, balaji@kluniversity.in, johnphilip@kluniversity.in

\begin{abstract}
This paper implements sigma delta analog to digital converters that play a key role in ECG acquisition. In this paper we have implemented both fifth and sixth order sigma delta analog to digital converters and compared the parameters like SNR, Nodal spectrum analysis, Integrated power analysis with respect to same frequency given to both fifth and sixth order circuits. In this paper we observed the increase in power consumption as the order increases. For 5 th order Sigma Delta ADC, we observed SNR of $74.8532 \mathrm{~dB}$. Similarly, for 6th order ADC the SNR observed was $75.5813 \mathrm{~dB}$.
\end{abstract}

Keywords: Sigma-Delta, ADC, ECG, SNR, acquisition 


\title{
PV Variations of Pulsed Latch Circuits
}

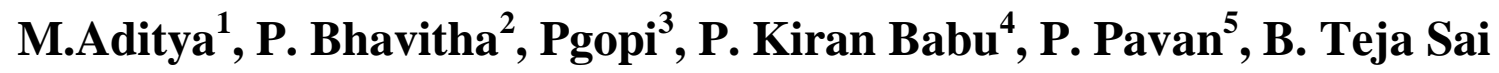 Varma $^{6}$}

Department of ECE, Koneru Lakshmaiah Education Foundation, Vaddeswaram, A.P., India522502

aditya@kluniversity.in,

\begin{abstract}
In this paper we studied the change in the result or action of different PV variations on the behavior of Pulsed Latches by taking the reaction on both the Pulser and the Latch. Pulsed Latches are gaining reduced delay and power consumption in low power ASIC design technology. They provide another sequential element with high performance and low area and power consumption, taking advantage of both latches and flip-flop features. This process implements the pulser by using MUX based pulser design. This design approaches are presented to improve the quality of Pulsed Latches circuits by using their main importance of high performance, low power, and small area. The proposed design has low power variation when operating at normal supply voltage.
\end{abstract}

Index Terms: Pulsed latches, variability, process variation, scaling of voltage, less power. 


\title{
Design of Magnitude Comparator Using Adiabatic Logic in
}

\section{Ecrl}

\author{
${ }^{1}$ K. Priyanka, ${ }^{2}$ P. Sathwik, ${ }^{3}$ S. Sai Rahul, ${ }^{4}$ Inumula Veeraraghava Rao \\ Department of ECE, Koneru Lakshmaiah Education Foundation, Vaddeswaram, A.P., India- \\ 522502 \\ 160040789@kluniversity.in, 160040670@kluniversity.in, 160040789@kluniversity.in, \\ raghva.inumula@kluniversity.in
}

\begin{abstract}
Efficiency of energy has become a major design to involve in high performance and mobile computer systems in this paper, a 2-bit comparator circuit is proposed by using with ECRL (Efficient Charge Recovery Logic). The term adiabatic is thermodynamics process that which recovery of energy and no exchange of energy occurs between system and environment. This design has improvement in power delay product from $65.76 \%$ to $83.02 \%$ at $1 \mathrm{~V}$ to $2 \mathrm{~V}$ when it is compared with the conventional design. The power dissipation will be reduced in adiabatic logic comparing with conventional CMOS logic. Recreation results uncover that the 2-bit ECRL (Efficient Charge Recovery Logic) comparator is $14.4 \%$ increasingly effective in power utilization when contrasted with the CMOS comparator, though a 2-bit PFAL comparator is $56.4 \%$ more effective in power utilization than the CMOS comparator. This result carried on Mentor Graphics. In adiabatic circuit the charge put away in load capacitor is recouped while in ordinary CMOS it is moved to ground which causes wastage of vitality. Adiabatic logic offers an approach to reuse the vitality put away in the load capacitors instead of the conventional method for releasing the capacitors to the ground and squandering this vitality. Results show a noteworthy improvement as far as PDP for proposed configuration as looked at to existing traditional plans.
\end{abstract}

Index Terms: ECRL, CMOS Logic, Adiabatic Logic, PDP 


\title{
International Journal for Innovative
} Engineering and Management Research

A Peer Revieved Open Access International Journal

DESIGN OF XOR and XNOR Gates Based on Full Adder Circuits

\section{${ }^{1}$ V. Kavya Chowdary, ${ }^{2}$ K. Sai Nishitha, ${ }^{3}$ V. Naveen Sai, ${ }^{4}$ Inumula Veeraraghava Rao}

Department of ECE, Koneru Lakshmaiah Education Foundation, Vaddeswaram, A.P., India522502

kavya24c@gmail.com, sainishitha99@gmail.com, naveensaicea@gmail.com, raghva.inumula@kluniversity.in

\begin{abstract}
This paper has a XOR / XNOR gate circuits produces separate and establishes a simultaneous XOR - XNOR function. Due to stub by yield capacity and short-circuit energy dissipation, the power utilization and latency of these circuits is increasing A new one-bit adder hybrid circuit is chosen built on the effective gates of xor, xnor or xor / xnor. Each circuit is preferable because of its own advantages as it is known for its high speed, low current drain, short delay product (PDP), galvanic ability, etc. Simulations of the planned models were carried out using Mentor Graphics to see the quality of these projects. The simulation results are based on the 130-nm CMOS engineering design. A recent technique of transistor sizing is implemented to improve the circuits ' PDP. Today, predominant electronic systems are treated separately in every part of life and the usage of portable devices has been increased tremendously. These devices require to have less power utilization and high speed. While designing a system, power utilization is a parameter which is to be rectified for better system performance. The efficiency that depends on the execution of divider, comparator, multiplier, and adder which comes under arithmetic circuit which in turn shows the impact on numerous digital applications.
\end{abstract}

Index Terms: Transistor sizing method, PSO, XOR-XNOR, Output driving capability, DPL 
QCA Design and Performance Analysis of Binary to Excess Code Converter

\title{
${ }^{1}$ John Philip B, ${ }^{2}$ Raghavarao I, ${ }^{3}$ SSS Kalyan, ${ }^{4}$ Aditya M
}

Department of ECE, Koneru Lakshmaiah Education Foundation, Vaddeswaram, A.P., India522502

1 johnphilip@kluniversity.in, ${ }^{2}$ raghvainumula@kluniversity.in, ${ }^{3}$ ssskalyan@kluniversity.in, aditya@kluniversity.in

\begin{abstract}
An efficient QCA Excess-3 code converter has been proposed for QCA. The proposed circuits can be efficiently used for data communication systems for reliable information communication at nanoscale. In CMOS devices, many complications arise such as quantum effects, short channel effects and power dissipation, when scaling down to submicron level. To overcome the limitations of CMOS devices, Quantum-Cellular Automata (QCA) technology is implemented to design the code converters. A well-optimized conversion technique like N-bit (typically 4-bit, 8-bit, 16-bit, 32-bit and 64-bit) Binary-to-Gray and a 4-bit Excess-3 code converter is explored for implementation using QCA. Attempt has been made to enhance the performance of conventional 4-bit Binary-to-Gray code converter. The proposed 4-bit Binary-to-Gray code converter has been compared with conventional designs and achieves better circuit performance in terms of area, circuit complexity and clock delays as depicted. A novel N-bit Binary-to-Gray code converter (for $\mathrm{N}=8,16,32$ and 64 ) has also been proposed in this paper. The proposed design enjoys the features of lesser area and reduced circuit complexity. Moreover, the proposed N-bit Binary-to-Gray code converter consumes very less clock delays as depicted in this paper. Efficient methods of interconnections for pipeline architecture structures have been considered. The design and simulation results of present work have verified using QCA Designer tool. The work conducted in this paper thus opens a wider path for digital circuit design with smaller circuit dimensions.
\end{abstract}

Keywords: Quantum-Cellular Automata, Binary to Gray, Code Converter, CMOS Devices, optimization. 


\title{
Validation of Rain Attenuation Time Series Synthesizers to Predict the Artificial Rain Attenuation for Propagation Impairments in Satellite Communications
}

\section{${ }^{1}$ John Philip B, ${ }^{2}$ Hashvath M, ${ }^{3}$ Jitendra Y, ${ }^{4}$ Maneesha Kolla}

\author{
Department of ECE, Koneru Lakshmaiah Education Foundation, Vaddeswaram, A.P., India- \\ 522502 \\ 1 johnphilip@kluniversity.in, ${ }^{2}$ hashvathshaik23@gmail.com, ${ }^{3}$ jitendrareddy168@gmail.com, \\ ${ }^{4}$ maneesha.kolla@gmail.com
}

\begin{abstract}
Whenever the signal attenuation due to rain is discussed, various forms of proposals have been brought forward. There are several models of attenuation that can be seen in the literature in the past, many of them are updated and there are still others to go; several of them are ITU rain model, DAH, Lognormal, Assis, Flavin, Crane, SAM, Moupfouma, Yamada, Lin, and so forth. The communication field of ITU radio (ITU-R) is one of the three branches of the International Telecommunications Union (ITU), which is responsible for radio transmission and regulates regional radio spectrum and satellite orbit resources, as well as improving radio communication network quality with the goal of ensuring the efficient usage of spectrum. Depending on data obtained from the United States, the ITU-R offers an estimation of the total distribution of rainfall in the planet by splitting it into five regions. To find out the rain attenuation at any particular region, some predefined calculations are necessary. i.e. from which height rain is falling, the nature of rainfall, etc. All these calculations are provided by the International Telecommunication Union. Hence, we prefer to use the ITU-R model. To develop and test real time Fade Mitigation Techniques control algorithms, propagation time series are needed. An alternative to using real data collected from propagation experiments is to generate typical fading time series making use of climatological characteristics as well as geometrical and radio-electrical parameters of the link. The aim of the study presented in this paper is to validate time-series synthesizers relying on an enhanced ITU-R model.
\end{abstract}

Keywords: Time Series, Rain Attenuation, Prediction Models, Propagation Impairments, Satellite Communications, Fade Mitigation Techniques. 

Engineering and Management Research

A Peer Revieved Open Access International Journal

\title{
DESGIN AND IMPLEMENTATION OF FULL ADDER USING INTERCONNECTS FOR LOW POWER AND HIGH-SPEED APPLICATION
}

\author{
R. Agilesh Saravanan ${ }^{1}$ \\ Assistant Profesor ${ }^{1}$, Department of ECE, Koneru Lakshmaiah Education Foundation, \\ Vaddeswaram, A.P., India-522502. agileshece@gmail.com
}

\begin{abstract}
Nowadays power dissipation is a more important problem in dense electronic devices. This power dissipation majorly affected the battery backup. So energy efficiency has become a important concern in the portable equipment to get better performance with less power dissipation. However the various logics where introduced to reduce the size but the integration density is increasing due to that power consumption also increasing. In this paper the CMOS 2 bit Full adder using various Adiabatic logic such as Efficient Charge Recovery Logic (ECRL), Positive Feedback Adiabatic Logic(PFAL) without interconnects and with interconnects has been implemented and the power delay relationship by using interconnects also has been analyzed.
\end{abstract}

Keywords: ECRL, PFAL, Adiabatic, Positive Feedback, Full Adder 

Engineering and Management Research

A Peer Revieved Open Access International Journal

\title{
Direction of Arrival Estimation using Conventional Subspace Algorithms
}

\author{
R. Agilesh Saravanan ${ }^{1}$ \\ Assistant Profesor ${ }^{1}$, Department of ECE, Koneru Lakshmaiah Education Foundation, \\ Vaddeswaram, A.P., India-522502. agileshece@gmail.com
}

\begin{abstract}
In recent times, Direction of Arrival (DOA) Estimation study earns attention in array signal processing and it develops rapidly in several application such as sonar, radar, communication, biomedicine and seismology measurements. The self-adaption and spatial spectrum are the broad research area in array processing. The spatial spectrum estimation focused on the signal distribution in the space is received from all direction to receiver. To maintain accuracy in DOA estimation for the antenna array the basic knowledge is required for main beam, and side lobes pattern must be small to suppress signal from other direction. The overview of the Direction of Arrival (DOA) estimation based on classical Sum and delay beamformer, Minimum Variance Distortion Less Response (MVDR) technique, Min Norm technique and Multiple Signal Classification (MUSIC) is discussed by using the spatial spectrum parameters.
\end{abstract}

Keywords: MUSIC, DOA, MVDR, Min-Norm, Beamforming 


\title{
Fuzzy Utilization in Speech Recognition and its Different Application
}

\author{
J. Bennilo Fernandes ${ }^{1}$, Kasiprasad Mannepalli ${ }^{2}$ \\ Department of ECE, Koneru Lakshmaiah Education Foundation, Vaddeswaram, A.P., India- \\ 522502. bennij05@gmail.com
}

\begin{abstract}
Talk affirmation is one among the basic zones in cutting edge talk process. The examination of talk affirmation may be a bit of an examination for "artificial intelligence" machines that may "hear" and "appreciate" the verbally communicated data. The customary ways for talk affirmation like HMM and DTW, are outrageously inconvenient and time excellent. As such formal Fuzzy justification may be an endeavor in cutting edge talk process for the convincing portrayal of talk affirmation in a couple of utilization. The approach masterminded in the midst of this paper streamlines the utilization of fuzzy in talk affirmation and make the data dealing with time shorter. The case considered in the midst of this paper is that the least mind boggling, i.e., the example of speaker dependence, little vocabulary and disconnected words. There are various spectral and common choices isolated from human talk. The present ways for tendency acknowledgment from voice use basically MFCC and Energy feature. This paper briefs an overview concerning the present work on talk feeling ID strong for completing more examination by feathery approach.
\end{abstract}

Index Terms: Emotional Speech Recognition, Fuzzy, HMM, NN, Applications. 


\title{
Emotion Recognition Using KNN
}

\section{Bennilo Fernandes. J ${ }^{1}$, Ch. Bhargavi ${ }^{2}$, S. Arshad ${ }^{3}$, G. Sai Kumar ${ }^{4}$, G. Sandeep $^{5}$}

Department of ECE, Koneru Lakshmaiah Education Foundation, Vaddeswaram, A.P., India522502. bennij05@gmail.com

\begin{abstract}
The human speech will offer totally different styles of records regarding the speaker and speech. The emotional state recognition helps an individual to identify their health conditions and mental stress. In this paper different emotions like happy, angry, fear, sad \& normal is examined for recognition. With this approach, a new database is collected form 8 students in different combinations like male \& female and utilized for analyzing. For any speech identification or recognition feature extraction and classification acts as the key factor, in this paper MFCC based Vector quantization is used for extracting the features and KNN classification for recognition accuracy is utilized. From the feature extraction of MFCC, the simulation results show $84.3 \%$ of overall accuracy $\&$ in which emotional state like - Normall gives high recognition rate of $90 \%$.
\end{abstract}

Index Terms: Speech Recognition, MFCC, Vector Quantization, KNN. 


\title{
EFFICIENT ECG SIGNAL COMPRESSION AND TRANSMISSION ALGORITHM USING DUAL ENCODING TECHNIQUE
}

\author{
Nama Ajay Nagendra \\ Department of ECE, Koneru Lakshmaiah Education Foundation, \\ Green Fields, Vaddeswaram, A.P., India-522502. \\ E-mail: ajay@kluniversity.in.
}

\begin{abstract}
This paper reports a productive electrocardiogram (ECG) signal pressure and transmission calculation dependent on mix of two encoding strategies with discrete cosine change. The proposed method gives high pressure proportion (CR) and low percent root-mean-square contrast (PRD) values. For execution assessment of the proposed calculation 46 records of ECG signals are taken from MIT-BIH arrhythmia information base. Each record of ECG signal is inspected at testing recurrence of $360 \mathrm{~Hz}$ more than 11-piece goal. To change the sign from time area to recurrence space, discrete cosine change (DCT) has been utilized which compacts energy of the sign to bring down request of recurrence coefficients. After quantization of change coefficients signals are encoded utilizing double encoding method which comprises of run length encoding and Huffman encoding. The double encoding procedure packs information essentially. The proposed calculation offers normal estimations of CR, PRD and quality score (QS) of 29.36, 1.31 and 23.06 separately.
\end{abstract}

Index Terms - electrocardiogram, encoding, discrete cosine, quality score, mean square error, Huffman. 


\title{
Smoothing and optimal compression of encrypted gray scale images
}

\author{
Nama Ajay Nagendra \\ Department of ECE, Koneru Lakshmaiah Education Foundation, \\ Green Fields, Vaddeswaram, A.P., India-522502. \\ E-mail: ajay@kluniversity.in.
}

\begin{abstract}
Compression efficiency of encrypted real-world sources such as images can be improved by exploiting source dependency. Lossless compression of encrypted images can be achieved through Slepian-Wolf coding. In this correspondence, we propose a resolution progressive compression scheme which compresses an encrypted image progressively in resolution, such that the decoder can observe a low-resolution version of the image, study local statistics based on it, and use the statistics to decode the next resolution level. Good performance is observed both theoretically and experimentally. We deploy smoothing for images at the receiver to mitigate noise.
\end{abstract}

Index Terms - compression, encrypted, Slepian-wolf, resolution, noise 


\title{
DETECTION OF DENGUE DISEASE BY USING MEMS
} BIOSENSOR

\author{
Shameem Syed*, Chandan motamarri, Charitha Alagadda \\ Department of ECE, Koneru Lakshmaiah Education Foundation, Vaddeswaram, A.P., India- \\ 522502. \\ *E-mail ID: shameemsyed@kluniversity.in
}

\begin{abstract}
Micro-Electro-Mechanical (MEMS) is a fast technology that carried different ideas in the fields like biomedical. MEMS got many applications in biomedical. Dengue is a break borne fever disease which is caused by dengue virus. This can be detected using MEMS bio-sensor. In this paper we use cantilever mechanism for the early stage detection of the disease. Here we use antibodies that are the bio-elements in the biosensor. The cantilever simulation result includes displacement and capacitance measurement which is the best method for dengue detection. Based on the amount of the displacement we can identify the severity of the disease.
\end{abstract}

Index Terms: biosensor, capacitance, displacement, antibody, and dengue. 

Engineering and Management Research

A Peer Revieved Open Access International Journal

\title{
CAPACITANCE BASED PIEZOELECTRIC ENERGY HARVESTER
}

\section{Shameem Syed*, Rama Krishna Prasad, G Bhavya Lahari, T Tarun}

Department of ECE, Koneru Lakshmaiah Education Foundation, Vaddeswaram, A.P., India522502.

*E-mail ID: shameemsyed@kluniversity.in

\begin{abstract}
Now-a-days the utilization of energy in all the fields raised to saturation. But, the resources presently available are not sufficient for utilization. So, it is essential to use the available natural resources and convert them into the utilizable form called energy Harvesting. As, the MEMS are the reliable low power structure, here we are proposing a new methodology with piezoelectric based energy harvesters. The simulation was done with aluminum, PZT and silicon. For the three materials the power analysis and the sensitivity are calculated and simulated. Among the three PZT gives low power operation and highly sensitive. The main advantage of the structure is the variation in the frequency caused by the intensity of the light radiation that incident on the PZT material. It is also observed that the sensitivity remains constant even though there will be a lot of variation in the frequency. The piezoelectric energy harvester cantilever arrayed beam produces power of 0.4496 Watts. The entire structure of cantilever is designed, analyzed and simulated using COMSOL MULTIPHYSICS 5.0 version.
\end{abstract}

Index Terms: PZT, sensitivity, energy harvester, cantilever. 


\title{
DESIGN OF MAGNITUDE COMPARATOR USING ADIABATIC LOGIC IN ECRL
}

\author{
I. Raghava
}

Assiatant Professor, Department of Electronics and Communication Engineering, Koneru Lakshmaiah Education Foundation, Green Fields, Vaddeswaram, Andhra Pradesh, India-522502

\begin{abstract}
Efficiency of energy has become a major design to involve in high performance and mobile computer systems in this paper, a 2-bit comparator circuit is proposed by using with ECRL (Efficient Charge Recovery Logic). The term adiabatic is thermodynamics process that which recovery of energy and no exchange of energy occurs between system and environment. This design has improvement in power delay product from $65.76 \%$ to $83.02 \%$ at $1 \mathrm{~V}$ to $2 \mathrm{~V}$ when it is compared with the conventional design. The power dissipation will be reduced in adiabatic logic comparing with conventional CMOS logic. Recreation results uncover that the 2-bit ECRL (Efficient Charge Recovery Logic) comparator is $14.4 \%$ increasingly effective in power utilization when contrasted with the CMOS comparator, though a 2-bit PFAL comparator is $56.4 \%$ more effective in power utilization than the CMOS comparator. This result carried on Mentor Graphics. In adiabatic circuit the charge put away in load capacitor is recouped while in ordinary CMOS it is moved to ground which causes wastage of vitality. Adiabatic logic offers an approach to reuse the vitality put away in the load capacitors instead of the conventional method for releasing the capacitors to the ground and squandering this vitality. Results show a noteworthy improvement as far as PDP for proposed configuration as looked at to existing traditional plans.
\end{abstract}

Index Terms: 2-bit comparator circuit, ECRL, CMOS Logic 


\title{
Artifact Elimination in Thoracic electrical bioimpedance signals using new Normalized Adaptive Filters
}

\author{
S. Surekha, Md Zia Ur Rahman \\ Department of Electronics and Communication Engineering, Koneru Lakshmaiah Education \\ Foundation, Green Fields, Vaddeswaram, Andhra Pradesh, India-522502 \\ surekhasala@gmail.com,mdzr55@gmail.com
}

\begin{abstract}
Thoracic electrical bioimpedance (TEB) used for facilitating stroke volume from sudden cardiac arrest signals. It is a non -invasive method used monitoring cardiac outputs, measuring stroke volumes and to observe changes in hemodynamic parameters of volume of blood. While measuring volume of blood, TEB signal is contaminated with physiological and non-physiological signal artifacts. For avoiding these artifacts in this paper proposed an adaptive filter method for enhancing TEB Signals. Least Mean Square (LMS) algorithm is a basic adaptive method, but it is non stationary in nature and it has low convergence rate problems. Hence, Bias compensated Normalized Least Mean Square (BC NLMS) algorithm is proposed, then it check initially for stability in terms of mean deviation analysis and mean square deviation analysis. Depending in this analysis, noisy input variance estimation and variable step size are taken into consideration for better performance in terms of reduced steady error rate, good stability and convergence improvement. In this paper we present various adaptive noise cancellers (ANCs) for the elimination of artifacts from TEB signals. Also, in simulation results, artifacts are eliminated from noisy input signal and it performs well when compared to exiting methods.
\end{abstract}

Index Terms: TEB, BC NLMS algorithm, ANC 


\title{
Energy Detection for Spectrum Sensing in Medical Telemetry Networks using Modified NLMS algorithm
}

\author{
S. Surekha ${ }^{1}$, Md. Zia Ur Rahman ${ }^{2}$ \\ Department of Electronics and Communication Engineering, Koneru Lakshmaiah Education \\ Foundation, Green Fields, Vaddeswaram, Andhra Pradesh, India-522502 \\ surekhasala@gmail.com,mdzr55@gmail.com
}

\begin{abstract}
ABSTARCT
In a clinical scenario when the patient is far from the hospital, the patient is not treated in time. In that scenario, the medical telemetry network plays a vital role. So, it is intended to develop a medical body area network for remote health care monitoring utilizing cognitive radio methods. A Medical Body Area Network (MBAN) consists of multiple sensor nodes, these nodes may be wearable and implantable devices and by using this device we get the patient physiological data and each having the capability of sampling, processing and communicating with vital signals. Medical Telemetry is a technology, it involves information processing and communication technologies to utilize in healthcare services. In wireless communications, medical telemetry plays a vital role to use in health care applications like telemedicine, remote patient monitoring, etc., Spectrum sensing is one of the cognitive radio methods, it can be used in the field of medical telemetry. Spectrum sensing using an energy detection technique can be used in medical telemetry for health care monitoring. The proposed implementation finds an immediate application in the development of smart cities and smart hospitals. Here, we use a Modified Normalized LMS algorithm for this purpose. Problems of energy detection and measurement lead to spectrum sensing in a telemetry network that can be solved by the proposed method. Results show that the performance of Modified Normalized LMS gives better simulations in terms of detection probability and false alarm.
\end{abstract}

\section{Index Terms: MBAN, Medical Telemetry, LMS Algorithm}




\title{
International Journal for Innovative
} Engineering and Management Research

A Peer Revieved Open Access International Journal

DESIGN OF XOR and XNOR Gates Based on Full Adder Circuits

\author{
I. Raghava \\ Department of Electronics and Communication Engineering, Koneru Lakshmaiah Education \\ Foundation, Green Fields, Vaddeswaram, Andhra Pradesh, India-522502
}

\begin{abstract}
:
This paper has a XOR / XNOR gate circuits produces separate and establishes a simultaneous XOR - XNOR function. Due to stub by yield capacity and short-circuit energy dissipation, the power utilization and latency of these circuits is increasing A new one-bit adder hybrid circuit is chosen built on the effective gates of xor, xnor or xor / xnor. Each circuit is preferable because of its own advantages as it is known for its high speed, low current drain, short delay product (PDP), galvanic ability, etc. Simulations of the planned models were carried out using Mentor Graphics to see the quality of these projects. The simulation results are based on the 130-nm CMOS engineering design. A recent technique of transistor sizing is implemented to improve the circuits ' PDP. Today, predominant electronic systems are treated separately in every part of life and the usage of portable devices has been increased tremendously. These devices require to have less power utilization and high speed. While designing a system, power utilization is a parameter which is to be rectified for better system performance. The efficiency that depends on the execution of divider, comparator, multiplier, and adder which comes under arithmetic circuit which in turn shows the impact on numerous digital applications.
\end{abstract}

Index Terms: one-bit hybrid adder circuit, arithmetic circuit, Digital applications 


\title{
Classification of dress codes using CNN
}

\author{
D.Bhavana ${ }^{1}$, K. Kishore Kumar ${ }^{2}$ \\ Department of ECE, Koneru Lakshmaiah Education Foundation, Green Fields, \\ Vaddeswaram, Andhra Pradesh, India-522502
}

\begin{abstract}
Individuals now live in a world encompassed by corporate culture like work environments and universities, schools, emergency clinics. Specifically, instructive organizations will guarantee the understudies to follow clothing regulation to get consistency among the understudies. It is a dull errand to the administration to distinguish the understudies who doesn't follow the clothing standard. Manual perception requires more human inclusion and it isn't workable for the whole day. More over the grounds is altogether enormous and checking understudies everywhere on the grounds physically expands the remaining burden. To determine those issues, the proposed model presents a neural organization-based characterization framework to distinguish and classify the understudies. Informational index comprises of 270 pictures of understudies and experts are utilized in the experimentation to perceive the clothing standard of people. The outcomes gave us exact desires using some graphical depictions similarly as periods of the various people.
\end{abstract}

Index Terms: Perception, Information Index, Graphical depictions 


\title{
Random number generator using Labview
}

\author{
D.Bhavana ${ }^{1}$, K. Kishore Kumar ${ }^{2}$ \\ ${ }^{1}$ Associate professor, Department of ECE, Koneru Lakshmaiah Education Foundation, Green \\ Fields, Vaddeswaram, Andhra Pradesh, India-522502
}

\begin{abstract}
ABSTARCT
Irregular number generator $[R N G]$ is use to produce arbitrary numbers between some random breaking point, RNG's are two sorts 1.True irregular number and 2.pseudo numbers. Genuine arbitrary numbers are not unsurprising by any numerical equation since they are fundamentally relies upon the climatic commotion, going to the pseudo numbers are predominantly utilized in the vast majority of PCs, this irregularity can be unsurprising by utilizing numerical recipe and it is fine numerous reasons, however it may not be arbitrary in the manner you expect on the off chance that you are utilized to dice rolls and lottery drawings. In this scaled down task we are doing RNG [pseudo numbers] by utilizing NI labview programming and producing arbitrary numbers by squeezing press catch and coming yield we are showing on seven section show. In the labview it simple to produce an irregular number by utilizing distinctive blockecs and primary favorable position of the labview is there is no need of any programming dialects like[c,c++,java,matlab].Main capacity of this undertaking is utilized for gaming and need number age and so on A grouping of uniform arbitrary numbers, which is produced inside the PC in a deterministic way, is frequently alluded to as a pseudo-irregular number succession Index Terms: Irregular Number Generation, Pseudo Numbers, Labview
\end{abstract}




\title{
IMPLEMENTATION OF C-ELEMENT AND D-ELEMENT EDGE TRIGGERED FLIP FLOP FOR VLSI APPLICATIONS
}

\author{
Sanath Kumar Tulasi ${ }^{1}$, Syed Inthiyaz ${ }^{2}$, GRK Prasad ${ }^{3}$, M. Siva Kumar ${ }^{4}$ \\ Department of Electronics and communication Engineering, Koneru Lakshmaiah Education \\ Foundation, Guntur, India-522502. \\ Email: tulasisanath@kluniversity.in
}

\begin{abstract}
In this paper the work has been carried out for attaining a very impressive achievement of low power digital circuits, without compromising in dual edge triggering phenomenon. In this work different strategies of static dual-edge triggered flip-flops (DET) which show exclusive circuit behaviour due to the usage of C-elements and D-element has been analysed. In D-element again, the two different categories like static and dynamic D-element were analysed in 130nm technology. One of the key elements used in asynchronous control circuits is the C-element. The performance metrics were analysed and tabulated their characteristics, through mentor graphics in 130nm technology.
\end{abstract}

Keywords- dual-edge triggered flip-flops (DET), C-elements, D-element, asynchronous control circuits 


\title{
Design ALU Based online BIST for Multi Word-Width RAM's
}

\author{
Sanath Kumar Tulasi ${ }^{1}$, Syed Inthiyaz ${ }^{2}$, GRK Prasad ${ }^{3}$, M. Siva Kumar ${ }^{4}$
}

Department of Electronics and communication Engineering, Koneru Lakshmaiah Education

Foundation, Guntur, India-522502. tulasisanath@kluniversity.in

\begin{abstract}
Memory cores are usually the densest portion with the smallest feature size in system-on-chip (SOC) designs. The reliability of memory cores thus has heavy impact on the reliability of SOCs. Transparent test is one of useful technique for improving the reliability of memories during life time. Transparent BIST schemes for RAM modules assure the preservation of the memory contents during periodic testing Symmetric Transparent Built-in Self Test (BIST) schemes skip the signature prediction phase required in traditional transparent BIST. Achieving considerable reduction in test time. Previous works or symmetric transparent BIST schemes require that a separate BIST module is utilized for each RAM under test. This approach, giver the large number of memories available in current chips, increase the hardware overhead of the BIST circuitry. In this work we propose a Symmetric transparent BIST scheme that can be utilized to test Rams. For 5 different word widths hence, more than one RAMs can be tested in a roving manner
\end{abstract}

Keywords- Random access Memory-RAM, Built-in-self test-(BIST), System-on-Chip-(SOC) 


\title{
A novel approach in optimal parameters selection for high
}

\section{performance and ultra-low power $\sum \Delta$ Modulator}

\author{
S. Vamsi Krishna \\ Department of Electronics and communication Engineering, Koneru Lakshmaiah Education \\ Foundation, Guntur, India-522502

\begin{abstract}
:
A linear model analysis is made for second to fourth order cascaded, single loop and hybrid sigma delta modulator architectures with a novel technique for deriving choice of parameters. Parameters obtained in this paper by considering the trade-offs between architectural to physical level implementation in top-down design approach, which guides efficient practical implementation of sigma delta modulator. With the proposed choice of parameters, the performance metrics of sigma delta modulators are verified in behavioural modelling and comparison of selected architectures are given for optimum selection of circuit topology and modulator parameters in design of SigmaDelta analog to digital converters.
\end{abstract}

Index terms: Sigma delta modulator, top-down design, behavioural modelling. 

Engineering and Management Research

A Peer Revieved Open Access International Journal

\title{
Systematic design and analysis of $3^{\text {rd }}$ order single loop $\sum \Delta$ Modulator for ECG signal processing
}

\section{S. Vamsi Krishna}

Department of Electronics and communication Engineering, Koneru Lakshmaiah Education Foundation, Guntur, India-522502

\begin{abstract}
This paper presents system level modelling of sigma delta modulator including circuit level non idealities in MATLAB/Simulink environment for low frequency applications. An overview of sigma-delta modulation technique in analog-to-digital conversion and implementation of $\sum \Delta$ analog-to- digital converters using different technology processes, architectural and circuit level methods explored. A third order single loop sigma delta modulator is designed and verified with careful selection of integrator weights to meet stable desired performance. The proposed designed achieved SNDR of $101.75 \mathrm{~dB}$ and 17 bit resolution. The simulation performed at behavioral level using Simulink based sigma delta simulator, the results obtained are accurate, by considering non ideal effects. The results obtained for the proposed cascaded sigma delta modulator compared with existing single loop and cascaded architectures and proved as efficient.
\end{abstract}

Index terms: analog-to-digital conversion, $\Sigma \Delta$ modulator, CMOS. 


\title{
Appearance Invariance Action Recognition with Multi Stream ConvNets on Unconstraint Indian Classical Dance Videos
}

\author{
E. Kiran Kumar \\ Department of Electronics and communication Engineering, Koneru Lakshmaiah Education \\ Foundation, Guntur, India-522502
}

\begin{abstract}
Human action recognition by machines has transformed into a machine learning problem using 2D image, video and 3D video action datasets. The current trend uses 2D videos, 3D depth maps and 3D skeletal data of common human motions for recognition. In this work, we propose a mixed spatial - temporal ConvNet to recognize complex human actions present in Indian classical dance performances. The datasets are constructed from 5 songs of an Indian classical dance form with 5 different dancers in two modes called offline and online. Offline videos are constructed in lab and online videos are from YouTube. The proposed CNN architecture is built with 4 streams of dataflows, two for extracting spatial information and the other two for temporal information. RGB video frames and graph based visual saliency maps generate spatial data. Gaussian mixture modelbased frame subtraction and optical flow maps activate temporal streams. The CNN architecture is built with 8 Conv layers and 2 fully connected layers. Two 6GB GPU's and 4 CPU's train the proposed $\mathrm{CNN}$ on 50 dance videos. Each dance video song is subdivided into 56 classes based on the song lyric and the corresponding dance pose. The proposed CNN architecture is tested using our Indian classical dance database (online \& offline) and other publicly available 2D action datasets such as KTH, UCF101, HMDB51, G3D and NTU. The results show a high degree of classification accuracy of $98.99 \%$ compared to the state-of-the-art multi stream CNN models.
\end{abstract}

Index Terms: ConvNet, CNN Architecture, multi stream CNN models 


\title{
A Spatial Two Stream CNNs for Human Action Recognition
}

\section{from Real Time Video Sequences}

\author{
E. Kiran Kumar \\ Department of Electronics and communication Engineering, Koneru Lakshmaiah Education \\ Foundation, Guntur, India-522502
}

\begin{abstract}
The identification of human action or behaviour is very much discussed through the use of computer vision and artificial intelligence. Various HAR strategies were proposed by many researchers to extract the action features in order to identify the action accurately. Convolutional neural networks (CNNs) are most powerful to perform recognition. This paper brings out the importance of spatial information in the action classification tasks. A two-stream spatial convolutional neural network is proposed in this paper to recognize the human actions from large video datasets. One stream of CNN is a RGB based spatial stream which is fed with the spatial information of raw RGB frames. The second stream of the network is GBVS based spatial stream, fed with the graph based visual saliency maps. Various feature fusion techniques were adopted to combine the outputs of both the spatial streams. The proposed method is tested on popular benchmark human action datasets namely, KTH, UCF101, HMDB51, G3D and NTU RGB-D to know the performance of the proposed method and it found works well. Reasonable recognition rates were observed on all the data sets.
\end{abstract}

Index Terms: Artificial Intelligence, CNN, spatial Information, RGB frames 


\title{
Speaker identification in variable health conditions
}

\author{
Kasiprasad Mannepalli ${ }^{1}, \underline{\text { Neeharika, G.M. }} .{ }^{1}, \underline{\text { Raviteja, G.S.V. }}{ }^{1}, \underline{\text { Jahnavi, S. }}{ }^{1}$
}

Department of ECE, Koneru Lakshmaiah Education Foundation, Vaddeswaram, A.P., India522502

Email: Kasiprasadm@kluniversity.in

\begin{abstract}
This work makes a verification regarding person identification (PI) in variable health conditions like cold and cough from the attitude on sensible frameworks. Less quantity of knowledge is enough for consumer comfortableness to verify speaker. Moreover, decrease in speech information influences the PI execution that turns into a worry for user usage. Here, varied information things for PI are investigated, and enough data with restricted check information is displayed as a perfect structure for theframeworks. numerous investigations are created victimization within the purpose of read of up execution in varied health conditions like cold and cough. These investigations incorporate vocal tract narrowing component embrace person-specific acoustic-phonetic data, varied characteristics of speech supply include that convey elective/integral information from that sent by common mel-frequency cepstral constant highlights. At last, a structure is planned in mixture with the expressed investigations to possess a higher person verified in varied health conditions, that is a vital purpose if there's spare data.
\end{abstract}

Index Terms: Person Identification, Vocal tract, Acoustic- phonetic data 


\title{
Design of a fault tolerant adder for delay reduction in FIR
}

\author{
filter \\ Kasiprasad Mannepalli ${ }^{1}$, Varma, A. V.S.S ${ }^{2}$. \\ Department of ECE, Koneru Lakshmaiah Education Foundation, Vaddeswaram, A.P., India- \\ 522502 \\ Department of ECE, Srinivasa Institute of Engineering \& Technology, \\ Mummidivaram,Amalapuram A.P., India \\ Email: Kasiprasadm@kluniversity.in
}

\begin{abstract}
Basically, to minimize the failure charge in the system, we want to introduce the fault tolerant system. Because of more than one faults came about in the system, the machine increases the area. To adapt the adder architecture, one of kind algorithms are used in digital sign processing. By introducing the fault tolerant system, the reliability of the proposed machine will increase. So, in this paper we introduced the graph of fault tolerant razor flip flop the use of SKLANSKY adder for lengthen discount in FIR filter. The razor flip flop will expand the power effectivity of proposed system. This flip flop will save the data via latching the circuit. The SKLANSKY adder is the section of arithmetic good judgment unit. In proposed system, all bits are summed and accompanied to the fault tolerance system. This fault tolerance device will realize the error and supply environment friendly output. Hence in contrast to existed system, the proposed machine gives high overall performance and accuracy in phrases of delay.
\end{abstract}

Index Terms: Adder Architecture, Digital sign processing, SKLANSKY Adder, FIR Filter 


\title{
A Novel Method of Using Auxiliary Information for Compressing Encrypted Images
}

\author{
Kasiprasad Mannepalli ${ }^{1}$, Varma, A. V.S.S ${ }^{2}$. \\ ${ }^{1}$ Department of ECE, Koneru Lakshmaiah Education Foundation, Vaddeswaram, A.P., India-
} 522502

\begin{abstract}
This paper presents a novel technique for giving security by compacting the scrambled pictures dependent on assistant data. The proposed technique encodes the uncompressed input picture utilizing disarray encryption calculation and furthermore creates assistant data utilizing modulo256 encryption. The helper data produced is utilized for information pressure and furthermore for picture recreation of the first picture. The channel supplier packed the encoded data utilizing quantization cycle and it is completed with the assistance of some ideal boundaries got from a piece of helper data and pressure proportion bending standards. The packed information incorporates an encoded sub-picture, the quantized information, the quantization boundaries and the piece of scrambled helper data. The recipient reproduced the first picture by using the compacted encoded data with mystery key. The concealed pieces are extricated from scrambled encoded adaptation of parallel guide and afterward an approximated encryption picture will be resolved from decoded paired guide and assessed key. The proposed technique is executed in MATLAB and the presentation is likewise completed for different pictures. The recreation results show that the packed encoded picture is effortlessly remade at the recipient with high pressure proportion and better PSNR at low data transmission.
\end{abstract}

Index Terms: Scrambled Pictures, Disarray encryption, MATLAB, PSNR 


\title{
An effective Face Recognition method using Gabor Wavelet Transform
}

\author{
Ali Baig Mohammad ${ }^{1}$, A Rajesh Naidu² \\ ${ }^{1}$ Assistant Professor, ECE Dept., Koneru Lakshmaiah Education Foundation, Vaddeswaram, AP, \\ India-522502 \\ ${ }^{2}$ Assistant Professor, ECE Dept., Ramachandra College of Engineering, Eluru, Andhra Pradesh, \\ India.
}

\begin{abstract}
Face recognition is emerging as an active research area with numerous commercial and law enforcement applications. Automated face recognition is a rapidly growing field that uses computer algorithms to determine the similarity between two face images. Although existing methods performs well under certain conditions, the illumination changes, out of plane rotations and occlusions remain as challenging problems. One of the major challenges in face recognitions to extract features from face images varying in facial expression, varying in lighting condition and varying in poses. The objective of this paper is to present the recognition of face images based on Gabor wavelets. The proposed algorithm deals with two of these problems, namely Pose and illumination changes. In our method, Gabor wavelet transform is used for facial feature vector construction due to its powerful representation of the behaviour of receptive fields in human visual system (HVS). The method is based on selecting peaks (high- energized points) of the Gabor wavelet responses as feature points. Compared to predefined graph nodes of elastic graph matching, our approach has better representative capability for Gabor wavelets. The feature points are automatically extracted using the local characteristics of each individual face to decrease the effect of occluded features. Performance comparison of recognizing face images taken under varying, varying lighting condition and varying poses are presented. Experimental results of Gabor wavelets for face recognition under varying lighting and poses conditions are provided. For experiments face images from the Stereo pair are used and, we have used Support Vector Machine (SVM) classifier for recognition of images. In our paper, we compare various color models and choose a color model that can best represent faces.
\end{abstract}

Index Terms: Face Recognition, HVS, Gabor wavelet transform, SVM classifier. 


\title{
Design of Nanoscale Square Ring Resonator Plasmonic Filter using MIM for Dual Band Applications
}

\author{
Surendra Kumar Bitra ${ }^{1}$ and Sridhar $\mathbf{M}^{2}$ \\ Department of ECE, Koneru Lakshmaiah Educational Foundation, Vaddeswaram, Guntur \\ District, Andhra Pradesh, India. \\ Email: bitrasurendrakumar@gmail.com
}

\begin{abstract}
:
In this paper, Metal-Insulator-Metal (MIM) based single and dual-band plasmonic bandpass filters (BPF) design and analysis is presented. The basic design comprises of a Square Ring Resonators (SRR). The SRR is coupled using straight waveguide with gap and Coupled feed line for single and dual-band operations, respectively. The straight waveguide SRR is responsible for a single band operating wavelength $(1355 \mathrm{~nm}$, i.e. $221.24 \mathrm{THz})$. The coupled feed line is used for dualband operating wavelengths, $1300 \mathrm{~nm}(230.6 \mathrm{THz})$ and $1600 \mathrm{~nm}(187.37 \mathrm{THz})$. Design and simulations are carried out using Finite Difference Time Domain (FDTD) based solverembedded in the Computer Simulation Technology (CST) Microwave Studio suite. The proposed filters are used for plasmonic single and dual-band bandpass filter (BPF) applications in photonicintegrated circuits (PIC's).
\end{abstract}

Keywords: MIM, Photonic Integrated Circuits, SRR and BPF 

Engineering and Management Research

A Peer Revieved Open Access International Journal

\title{
Concurrent Dual and Triple Band Square Ring Resonator Base-Band Filter using MIM for Plasmonic Applications
}

\section{Surendra Kumar Bitra ${ }^{1}$ and Sridhar $\mathbf{M}^{2}$}

Department of ECE, Koneru Lakshmaiah Educational Foundation, Vaddeswaram, Guntur

District, Andhra Pradesh, India.

Email: bitrasurendrakumar@gmail.com

\begin{abstract}
:
Ring resonators are capable of providing high-quality factors with low insertion loss, which are the factors for considering it as a potential technique of guiding signal in the nanometer wavelengths. In this paper, a Nanoplasmonic configuration of a resonator comprising of the square ring known as square ring resonator (SRR) is designed and analyzed for multiple band characteristics. The performance analysis of two different structures of the square ring resonators are presented in terms of the simulation reports like reflection and transmission coefficients, and field distribution plots. The designed Band Pass Filter (BPF) expressed excellent performance in the optical bands and hence are best suitable for Photonic Integrated Circuit (PIC) applications.
\end{abstract}

Keywords: BPF, Dual Band, MIM, PIC, SRR, Surface Plasmons, Triple Band. 


\title{
An Ultra-Wideband Band Pass Filter Using MIM Waveguide for Nano Scale Applications
}

\author{
Surendra Kumar Bitra ${ }^{1}$ and Sridhar $\mathbf{M}^{2}$ \\ Department of ECE, Koneru Lakshmaiah Educational Foundation, Vaddeswaram, Guntur \\ District, Andhra Pradesh, India. \\ Email: bitrasurendrakumar@gmail.com
}

\begin{abstract}
:
A T-stub Square Ring Resonator (SRR) based Ultra-Wide Band (UWB) Band Pass Filter (BPF) is studied and investigated in this letter. The proposed filter is based on coupled feed line is connected to T-stub SRR. Ultrawide band characteristics can be realized by adjusting T-stub lengths, and coupling gaps between both sides of waveguides and SRR. The characteristics of the T-stub SRR shows that the miniaturized UWB BPF can be operated at Terahertz (THz) frequencies. The proposed UWB filter is simulated and analyzed using finite differential time domain solver (FDTD) based Computer Simulation Technology (CST) studio suite. The resonance conditions are explained the transmission performance of the filter agrees with the simulated and theoretical calculations. The proposed filter is best suitable for electronic-photonic integrated circuits (EPICs).

Key words- Metal Insulator Metal (MIM), Ring resonator, Coupled Lines, Finite Differential Time Domain (FDTD), Ultra-Wide Band (UWB) and T-stubs.
\end{abstract}




\title{
MIM based Stepped Impedance Square Ring Resonator Dual-Band Band Pass Filter
}

\author{
Surendra Kumar Bitra ${ }^{1}$ and Sridhar $\mathrm{M}^{2}$ \\ Department of ECE, Koneru Lakshmaiah Educational Foundation, Vaddeswaram, Guntur \\ District, Andhra Pradesh, India. \\ Email: bitrasurendrakumar@gmail.com
}

\begin{abstract}
:
In this paper, a Metal Insulator Metal (MIM) based Plasmonic Stepped Impedance Square Ring Resonator (SI-SRR) Band Pass Filter (BPF) is designed and analysed for dual band applications. The MIM based SI-SRR is investigated using commercially available CST studio suite. The proposed SI-SRR is compact and low power requirements suitable for Photonic Integrated Circuits (PICs). The SI-SRR is operated in the wavelength's of $1317 \mathrm{~nm}(227.6 \mathrm{THz})$ and $1640 \mathrm{~nm}(182.8$ $\mathrm{THz}$ ) with appropriate reflection and transmission parameters. The stepped impedance stubs are used in the ring resonator for tunable operating bands. The proposed SI-SRR has wide applications in PICs
\end{abstract}

Keywords: MIM, SI-SRR, BPF and PICs. 


\title{
Analysis of Power Spectrum Density on Earthquake Data Using Modified Covariance Algorithm
}

\author{
A. Mounika Reddy ${ }^{1}$, B.Jayasree ${ }^{2}$, S.Koteswara Ra ${ }^{3}$,V.Lakshmi Bharathi ${ }^{4}$ \\ Department of ECE, Koneru Lakshmaiah Education Foundation, Green Fields, Vaddeswaram, \\ Guntur, Andhra Pradesh 522502. \\ Email: mounikaasam@gmail.com
}

\begin{abstract}
Seismic signals have very low Signal to Noise Ratio (SNR) due to ground roll noise. These signals undergo filtering and can be extracted by using seismogram. In this paper, FIR band pass filter is used to reduce noise. Modified Covariance is used to analyze the seismic signal spectrum and also for estimating traces of seismic signals.

Index Terms: Stochastic signal processing, Adaptive Signal processing, Seismology, Applied statistics.
\end{abstract}




\title{
Power Spectrum Estimation of Seismic Wave Using Periodogram Method
}

\author{
G.Visalakshmi ${ }^{1}$, L.Praveen ${ }^{2}$, Dr.K.S.Ramesh ${ }^{3}$, Dr.S.Koteswara Rao ${ }^{4}$,V.Lakshmi \\ Bharathi $^{5}$ \\ Department of ECE, Koneru Lakshmaiah Education Foundation, Green Fields, Vaddeswaram, \\ Guntur, Andhra Pradesh 522502. \\ Email: visali231996@gmail.com
}

\begin{abstract}
Volcanoes, Tsunamis and many other seismic sources produce sudden elastic waves that propagate through the Earth. The time of occurrence and the frequencies of these earthquake waves must be predicted to mitigate the damage. These parameters are attained from the power spectrum. In this article, Periodogram technique is used for the calculation of spectral density.Simulation is carried out and the results are presented.
\end{abstract}

Index Terms: Adaptive signal processing, Stochastic signal processing, Applied statistics, Precise estimation technique, Seismology. 


\title{
Spectral analysis of seismic signals using Burg algorithm
}

\author{
V. Ravi Teja ${ }^{1}$, U. Rakesh ${ }^{2}$, S. Koteswara Rao ${ }^{3}$, V. Lakshmi Bharathi ${ }^{4}$ \\ Department of ECE, Koneru Lakshmaiah Education Foundation, Green Fields, Vaddeswaram, \\ Guntur, Andhra Pradesh 522502. \\ Email: raviteja.vattikuti666@gmail.com
}

\begin{abstract}
Seismic signal has high noise and it must be filtered to extract the original seismic signal from the seismogram. In this paper FIR band pass filter is used to reduce the noise and Burg algorithm is used to process seismic signal for the estimation of seismic signal spectrum where burg minimizes the forward and backward prediction errors.
\end{abstract}

Index Terms: Applied Statistics, Adaptive signal processing, Stochastic signal processing, Seismology. 


\title{
International Journal for Innovative
} Engineering and Management Research

A Peer Revieved Open Access International Journal

\section{Underwater Target Tracking System Using Active Sonobuoys}

\author{
K. Bhargav Sri Sai ${ }^{1}$, K. Narashima Rao ${ }^{2}$, S. Koteswara Rao ${ }^{3}$, Kausar Jahan ${ }^{4}$ \\ Department of ECE, Koneru Lakshmaiah Education Foundation, Green Fields, Vaddeswaram, \\ Guntur, Andhra Pradesh 522502. \\ Email: raviteja.vattikuti666@gmail.com
}

\begin{abstract}
Surveillance of the targets done in two phases, one is radar (which is operated in air), another is sonar (which is operated underwater). These are operated in active mode. Sonar's range and bearing measurements are used to estimate the target position and velocity. In this paper by using Sonobuoy, observer tracks a target in underwater environment. Sonobuoy operates in active mode where it emits sound energy (pings) into the water and listen back echo before transmitting another pulse. The simulation is carried out in Matlab environment using Monte-Carlo statistical process. For smoothing of measurements and reduction of error in the predicted target motion parameters, extended Kalman filter algorithm is utilized.
\end{abstract}

Index Terms: Sonobuoy, Extended Kalman Filter, Bearing Angle, Estimation, Monte-Carlo method. 

Engineering and Management Research

A Peer Revieved Open Access International Journal

\section{Quad-Band Ring Loaded Circular Patch Antenna With Meander Line Slot For Wireless Applications}

${ }^{1}$ Mahesh Babu Kota, ${ }^{2}$ T.V. Rama Krishna, ${ }^{2}$ Ketavath Kumar Naik, ${ }^{1}$ E. E. Sai Yashwanth, ${ }^{1}$ G. Hanimi Reddy, ${ }^{1}$ K. Gowtam Chowdary

Department of Electronics and Communication Engineering, Koneru Lakshmaiah Education Foundation, Green Fields, Vaddeswaram, Andhra Pradesh, India-522502.

tottempudi@kluniversity.in

ABSTRACT:

In this paper, a quad band antenna with circular patch antenna loaded with annular ring with CSRR DGS is presented. To improve the impedance bandwidth, a meander line structure is etched on the patch and Complementary Split Ring Resonator (CSRR) structure on ground plane of the antenna. The proposed antenna has resonated at quad band for wireless applications. The operating frequencies are observed at $2.31 \mathrm{GHz}, 3.05 \mathrm{GHz}, 4.69 \mathrm{GHz}$ and $5.6 \mathrm{GHz}$ with reflection coefficients $-9.47 \mathrm{~dB},-22.80 \mathrm{~dB},-18.87 \mathrm{~dB}$ and $-25.27 \mathrm{~dB}$ respectively. The gain at the resonating frequencies are $2.67 \mathrm{~dB}, 3.74 \mathrm{~dB}, 4.5 \mathrm{~dB}$ and $4.31 \mathrm{~dB}$. The radiation patterns are also presented. The obtained frequency bands are suitable for wireless applications like WiMAX, WiBro and WLAN.

Index Terms: Circular patch, loaded annular ring CSRR, meander line, WiMAX, WLAN 


\title{
Dual Annular Ring Coupled Stacked Psi Shape Patch
}

\section{Antenna for Wireless Applications}

\author{
${ }^{1}$ Mahesh Babu Kota, ${ }^{2}$ T.V. Rama Krishna \\ Department of Electronics and Communication Engineering, Koneru Lakshmaiah Education Foundation, \\ Green Fields, Vaddeswaram, Andhra Pradesh, India-522502. \\ Email: tottempudi@kluniversity.in
}

\section{ABSTRACT:}

The paper aims to design and analyse an annular ring coupled stacked Psi shaped patch antenna with coplanar waveguide (CPW) feed technique operating for dual band frequency applications. The proposed model comprises of stacked Psi shapes resonating for lower order frequency. The second order resonating band was obtained through capacitively coupled overlapped annular rings. The geometrical dimensions of the proposed model are $3.5 * 2(\mathrm{~L} * \mathrm{~W})$ based on lower order resonating band. The design and simulations were performed using DS CST Microwave Studio suite. The model achieves dual resonant bands, (2.19- 2.68) GHz with impedance bandwidth 490 $\mathrm{MHz}$ and $(5.569$ - 6.09) $\mathrm{GHz}$ with $530 \mathrm{MHz}$ impedance bandwidth. The center frequencies are 2.42 $\mathrm{GHz}$ and $5.815 \mathrm{GHz}$ with return loss $-28.97 \mathrm{~dB}$ and $-28.99 \mathrm{~dB}$ respectively. The design exhibited a maximum gain of $5 \mathrm{~dB}$ with bidirectional and omni directional patterns in $\mathrm{E}$ and $\mathrm{H}$ planes. The axial ratio at the two resonating bands was less than $3 \mathrm{~dB}$. Parametric analysis was performed for reflection co-efficient on geometric variables like ground width, ground length and permittivity. From (3-5) GHz frequency range, a perfect notch band was also exhibited. Simulated and measured results have shown a good concurrence. The model was suitable for WLAN (Wireless Local Area Network) and ISM (Industrial, Scientific and Medical) Band applications.

Index Terms: Annular Ring, Stacked Psi Shape, WLAN, ISM Band 


\title{
CPW Fed Compact overlapped Annulus patch antenna with Incorporated Psi shapes for ISM Band and WLAN Applications
}

\author{
${ }^{1}$ Mahesh Babu Kota, ${ }^{2}$ T.V. Rama Krishna \\ Department of Electronics and Communication Engineering, Koneru Lakshmaiah Education \\ Foundation, Green Fields, Vaddeswaram, Andhra Pradesh, India-522502. \\ Email: tottempudi@kluniversity.in
}

\begin{abstract}
:
The paper proposes the design of economic and compact CPW (Co-Planar Waveguide) Fed

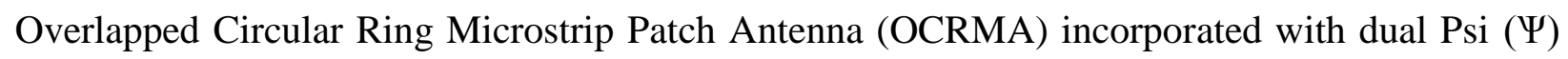
shapes for multiband operation. The designed model has geometrical dimensions of $0.28 \lambda_{0} * 0.16$ $\lambda_{0} * 0.0012 \lambda_{0}\left(\mathrm{~L}^{*} \mathrm{~W} * \mathrm{~h}\right)$ with respect to Zeroth Resonant Frequency (ZRF). The model resonates at $2.403 \mathrm{GHz}(\mathrm{ZRF})$ and $4.728 \mathrm{GHz}$ providing -10dB Impedance bandwidth of $770 \mathrm{MHz}$ and $1050 \mathrm{MHz}$ with fractional bandwidths of $32.08 \%$ and $22.2 \%$ respectively. Further, it provides a gain of $2.76 \mathrm{dBi}$ and $3.42 \mathrm{dBi}$ at the two resonant frequencies. The parameters like Return Loss $\left(S_{11}\right)$, gain, VSWR and axial ratio were analyzed to emphasize the performance of the patch antenna. A good consistency was observed between the measured and simulated results. The proposed model is suitable for WLAN $(2.4 \mathrm{GHz})$, ISM band $(2.45 \mathrm{GHz})$ and C-band $(4-8 \mathrm{GHz})$ applications.
\end{abstract}

Index Terms: Circular Ring, WLAN, ISM Band, C-band applications 


\title{
Circular monopole patch antenna Design with CSRR DGS for Wireless Applications
}

\author{
${ }^{1}$ Mahesh Babu Kota, ${ }^{2}$ T.V. Rama Krishna \\ Department of Electronics and Communication Engineering, Koneru Lakshmaiah Education \\ Foundation, Green Fields, Vaddeswaram, Andhra Pradesh, India-522502. \\ Email: tottempudi@kluniversity.in
}

\begin{abstract}
:
The paper presents design of a compact multiband circular monopole patch antenna with Complementary Split Ring Resonator (CSRR) as a Defected Ground Structure (DGS) on a FR-4 substrate suitable for wireless applications. The proposed antenna exhibits multiband operation with the inclusion of DGS structure. The antenna parameters like Return Loss $\left(\mathrm{S}_{11}\right)$, VSWR, Radiation Pattern, Gain were obtained through simulation and compared with the measured values. The parametric analysis was performed for the dimensions of the CSRR structure. It is observed that the proposed model has shown very good performance at the resonating frequencies $2.4 \mathrm{GHz}$, 4.01 GHz, 5.58 GHz, 7.07 GHz with impedance bandwidths (2.35-2.48), (3.95-4.09),(5.49-5.66), (7.01-7.16) $\mathrm{GHz}$ respectively. The peak gain obtained is $4.5 \mathrm{~dB}$ and the VSWR is maintained less than 2. The antenna exhibits monopole like radiation pattern for all the resonating bands making it suitable for the applications like WLAN, C- band Communications, WIMAX.
\end{abstract}

Index Terms: CSRR, Monopole, Wireless Applications. 


\title{
Machine Learning Techniques for Automatic Defect Detection in Frequency Coded Thermal Wave Imaging
}

\author{
V. Gopi Tilak ${ }^{1}$, V. S. Ghali ${ }^{1}$, A. Vijaya Lakshmi ${ }^{1}$ and B. Suresh ${ }^{1}$ \\ ${ }^{1}$ Infrared Imaging Center, Koneru Lakshmaiah Educational Foundation, Vaddeswaram, Andhra \\ Pradesh, India-522502. \\ E-mail.ID: gvs0raos@gmail.com
}

\begin{abstract}
Subsurface non-uniformity detection plays crucial role in deciding the strength of various industrial objects for future utilization. Frequency coded thermal wave imaging is emerging as a reliable non-destructive testing procedure to detect anomalies in a wide range of materials. This paper analyzes the performance of few supervised machine learning based classification techniques to detect the subsurface defects in quadratic frequency modulated thermal wave imaging. Experimentation has been carried over glass fiber and carbon fiber reinforced polymer materials embedded with Teflon patches and flat bottom holes having different sizes at varying depths. Three supervised classifiers such as Decision tree (DT), Support vector machine (SVM) and k-nearest neighbor (KNN) are used for defect detection. Signal to noise ratio and probability of defect detection concerned as figure of merits to qualify the detection capability and reliability of proposed techniques.
\end{abstract}

Index Terms: infrared thermography, fiber reinforced polymers, classifiers and quadratic frequency coded thermal wave imaging. 


\title{
Clustering in Non-linear Thermal Wave Imaging for Defect Detection
}

\author{
V. Gopi Tilak ${ }^{1}$, V. S. Ghali ${ }^{1}$, A. Vijaya Lakshmi ${ }^{1}$ and B. Suresh ${ }^{1}$ \\ ${ }^{1}$ Infrared Imaging Center, Koneru Lakshmaiah Educational Foundation, Vaddeswaram, Andhra \\ Pradesh, India-522502. \\ E-mail.ID: gvs0raos@gmail.com
}

\begin{abstract}
:
Defect characterization in the test object from the raw thermal response plays a vital role in assessing the reusability of the test object. On the other hand, assessing the biomedical properties of bone provide early diagnosis of osteoporosis. In recent past, non-linear thermal wave imaging gained interest as a viable thermal wave imaging technique. This paper introduces unsupervised clustering algorithms such as K-means and Fuzzy C means clustering to detect subsurface anomalies in composite structures and density variations in bone sample. Principal component analysis is used as Fuzzification technique in fuzzy c-means algorithm. Thermal response from the experimental carbon fiber reinforced polymer sample and simulated bone sample are used to validate proposed methodology. Signal to noise ratio (SNR) is taken as performance merit and on comparison, we conclude Fuzzy c-means provides better detection and characterization of defects compared to K-means clustering for QFMTWI.
\end{abstract}

Index Terms: Infrared thermography, fiber reinforced polymers, K-means clustering and Fuzzy c-means clustering. 


\title{
Orthogonal Projection for Enhanced Subsurface Detection in Non-stationary Thermal Wave Imaging
}

\author{
V. Gopi Tilak ${ }^{1}$, V. S. Ghali ${ }^{1}$, A. Vijaya Lakshmi ${ }^{1}$ and B. Suresh ${ }^{1}$ \\ ${ }^{1}$ Infrared Imaging Center, Koneru Lakshmaiah Educational Foundation, Vaddeswaram, Andhra \\ Pradesh, India-522502. \\ E-mail.ID: gvs0raos@gmail.com
}

\begin{abstract}
:
Whole field, non-contact and non-invasive evaluation capabilities recommend active thermography as a reliable non-destructive testing modality. Subsurface anomaly is identified by using the localized temperature variation over the object. The weak and noise embedded temperature response obtained in detection demands various processing approaches. A suitable processing, which enhances the signal to noise ratio or uncorrelated noise by separating the noise from other features facilitates enhanced detail extraction. In the recent past, Pulse compression used in RADAR and time frequency processing methods or feature separation methods enhances detectability and depth resolution of subsurface anomalies as well. This interdisciplinary research contribution introduces feature separation based detection through orthogonal projection for nonstationary thermal wave RADAR to cater the above requirements and quantify it in terms of signal to noise ratio.
\end{abstract}

Index Terms: Infrared thermography, fiber reinforced polymers, Thermal wave radar and Orthogonal projection. 


\title{
Composite Inspection through Quadratic Frequency Modulated Thermal Wave Imaging with Spectral Reshaping
}

\author{
V. Gopi Tilak ${ }^{1}$, V. S. Ghali ${ }^{1}$, A. Vijaya Lakshmi ${ }^{1}$ and B. Suresh ${ }^{1}$ \\ ${ }^{1}$ Infrared Imaging Center, Koneru Lakshmaiah Educational Foundation, Vaddeswaram, Andhra \\ Pradesh, India-522502. \\ E-mail.ID: gvs0raos@gmail.com
}

\begin{abstract}
:
Composite materials outperformed the usage of various metals in industrial applications due to their extreme mechanical properties. On the other hand, defect detection in composite structures recommends various non-destructive testing techniques. Active infrared thermography became a viable non-destructive testing technique due to its whole field, non-contact, remote and safe inspection capabilities. In the recent past, Quadratic frequency modulated thermal wave imaging (QFMTWI) is gaining interest for active thermography due to its depth resolution capability. This contribution introduces a post processing technique based on spectral reshaping of obtained thermal response. The effect of spectral reshaping by employing Bartlett window on thermal response obtained from a carbon fiber reinforced polymer specimen excited by quadratic frequency modulated heat flux is analyzed using various post-processing techniques. The analysis is carried out by applying various post processing techniques on raw and windowed thermal response and Signal to noise ratio is taken as performance metric for comparison of results.
\end{abstract}

Index Terms: Infrared thermography, fiber reinforced polymers, Bartlett window and Spectral reshaping. 


\title{
Design of a Non-uniform Serpentine Asymmetric Cantilever RF-MEMS Shunt Capacitive Switch for RADAR Applications
}

\section{K.Srinivasa $\operatorname{Rao}^{1}$}

Department of ECE, Koneru Lakshmaiah Education Foundation, Guntur, Andhra Pradesh, India E-mail address: drksrao@kluniversity.in

\begin{abstract}
:
This paper presents the design and simulation of Cantilever type RF-MEMS Capacitive shunt switch with meanders and perforations. The main objective of this paper is to reduce the pull-in voltage and increases isolation of the proposed switch. Electro-mechanics and Solid-mechanics are used to simulate the proposed switch by using COMSOL Multiphysics software. The capacitance, switching time, stress analysis are calculated and compare both theoretical and simulation results of the proposed switch. By varying different beam thickness, materials, an air gap between the beam and signal line to calculate spring constant and pull-in voltage. The proposed switch having pull-in voltage is $1.371 \mathrm{~V}$, the dielectric material Si3N4 is to improve the capacitance analysis of the switch. The up and downstate capacitance is $7.073 \mathrm{fF}, 1.259 \mathrm{pF}$. The RF performance is simulated by using Ansoft HFSS tool, the switch performs at low frequencies at 1-4 GHz range. The return ( S11) and insertion loss ( $\mathrm{S} 12)$ of the proposed switch is $-30 \mathrm{~dB},-$ $0.0516 \mathrm{~dB}$ and the switch having good is isolation (S21) is $-37.5 \mathrm{~dB}$ at $1.5 \mathrm{GHz}$ frequency. The switch is suitable for radar and satellite communication applications.
\end{abstract}

Keywords Cantilever type shunt switch $\cdot$ Pull-in voltage $\cdot R F$-analysis $\cdot$ Comsol software and HFSS software 

Engineering and Management Research

A Peer Revieved Open Access International Journal

\title{
Electromagnetic Analysis of MEMS-Based Tunable EBG Bandstop Filter Using RF MEMS Switch for Ku-Band Applications
}

\begin{abstract}
K.Srinivasa Rao
Department of ECE, Koneru Lakshmaiah Education Foundation, Guntur, Andhra Pradesh, India

E-mail address: drksrao@kluniversity.in

ABSTRACT:

This paper presents an electromagnetic analysis of EBG Bandstop filter integrated with RF MEMS switch. The transmission line theory in microwave technology is used to analyze the proposed structure by studying isolation parameter of the filter. A fixed-fixed switch with low pull-in voltage of $4.05 \mathrm{~V}$ is designed and integrated on the signal line which is equidistant from the two EBG structures of filter. The filter resonates at $14.05 \mathrm{GHz}$ without integrating the switches and is shifted to $15.85 \mathrm{GHz}$ due to upstate capacitance of $41.56 \mu \mathrm{F}$ when integrated with the switch at on-state. The frequency is tuned to $15.27,14.5$ and $11.4 \mathrm{GHz}$ by actuating the beam to displace about 1, 2 and $3 \mu \mathrm{m}$. The switch produces a downstate capacitance of $19.11 \mathrm{pF}$ during offstate and tunes the resonant frequency to $11.4 \mathrm{GHz}$. Thus, the tuning of the proposed EBG Bandstop filter is achieved by using RFMEMS switches and is efficiently used for Ku-band applications. The proposed electromagnetic analysis is carried out using HFSS 13.0v FEM tool.
\end{abstract}

Keywords Electromagnetic analysis - EBG Bandstop filter • RF MEMS switch • Microwave technology • Transmission line theory 


\title{
Design and simulation of MEMS based capacitive pressure sensor for harsh environment
}

\begin{abstract}
K.Srinivasa Rao
Department of ECE, Koneru Lakshmaiah Education Foundation, Guntur, Andhra Pradesh, India E-mail address: drksrao@kluniversity.in

ABSTRACT:

This In this paper, we have designed and simulated a capacitive pressure sensor for harsh environment. The sensor is metal-insulator-metal type sensor in which the structure is designed using gold and the dielectric using silicon nitrate with thickness of $0.51 \mathrm{~m}$. The device achieves a linear characteristic response and high sensitivity. MIM consists of a circular clamped-edges gold membrane suspended over sealed cavity on the upper metal surface which is placed on the silicon substrate. The proposed MEMS capacitive pressure sensor is optimised and designed in COMSOL Multiphysics. The sensor illustrated with a proof mass $1401 \mathrm{~m}$ diameter, with air gap from 0.15 to $2 \mathrm{~lm}$ and pressure ranging from 200 to1500Mpa
\end{abstract}

Keywords presser sensors . harsh environment. metal-insulator-metal. high sensitivity. COMSOL Multiphysics 
Design and performance analysis of hybrid SPDT RF MEMS

\title{
switch
}

\section{K.Srinivasa Rao}

Department of ECE, Koneru Lakshmaiah Education Foundation, Guntur, Andhra Pradesh, India E-mail address: drksrao@kluniversity.in

\begin{abstract}
:
This paper presents the design and simulation of Hybrid type RF MEMS switch for satellite communication application. The Hybrid switch beam is having non-uniform meanders and holes. The gold material is used as a beam and CPW. The gap between the beam and dielectric is $31 \mathrm{~m}$. The design is based on the shunt and series configuration consisting of 2 capacitive switches and 2 Ohmic switches. The performance analysis is done by using the HFSS tool. The individual Ohmic switch having $31 \mathrm{~dB}$ isolation and capacitive switch having $67 \mathrm{~dB}$ isolation. The proposed Hybrid switch having $87 \mathrm{~dB}$ isolation when both switches are in the OFF state. During ON state, the switch shows the low insertion loss of- $0.18 \mathrm{~dB}$ and return loss of- $63 \mathrm{~dB}$ at $16 \mathrm{GHz}$
\end{abstract}

Keywords RF MEMS switch. Coplanar Waveguide (CPW). Electromagnetic analysis. Hybrid SPDT switch. 


\title{
Design and performance analysis of double cantilever type capacitive shunt RF MEMS switch
}

\author{
K.Girija Sravani, D.Prathyusha and K.Srinivasa Rao \\ Department of ECE, Koneru Lakshmaiah Education Foundation, Guntur, Andhra Pradesh, India \\ E-mail address: kondavitee.sravani@kluniversity.in
}

\begin{abstract}
:
This paper presents a novel structure of capacitance shunt type RF switch for 5G applications. The proposed RF MEMS switch is having Cantilever type designed with optimized dimensions to operate in V-band applications. The electromechanical analysis is done by using the COMSOL tool. The actuation voltage of the proposed switch is $10.5 \mathrm{~V}$ with the air gap of $1 \mu \mathrm{m}$ and gold as a beam material. The proposed switch with the meanders and perforations show the scattering parameters in HFSS software such as insertion loss $\left(\mathrm{S}_{12}\right)$ of $-0.033 \mathrm{~dB}$ and return loss $\left(\mathrm{S}_{11}\right)$ less than $-48 \mathrm{~dB}$ and the isolation $\left(\mathrm{S}_{21}\right)$ calculated in off-state as $-62 \mathrm{~dB}$ at $50 \mathrm{GHz}$.
\end{abstract}

Keywords : RF MEMS shunt switch $\cdot$ Pull-in voltage $\cdot$ RF-analysis 


\title{
A modified proposed capacitance model for step structure capacitive RF MEMS switch by incorporating fringing field effects
}

\author{
K.Girija Sravani ${ }^{1}$, Koushik Guha ${ }^{2}$ \\ ${ }^{1}$ Department of ECE, Koneru Lakshmaiah Education Foundation, Guntur, Andhra Pradesh, India \\ ${ }^{2}$ Department of ECE, NIT Silchar, Assam, India \\ E-mail address: kondavitee.sravani@kluniversity.in
}

\begin{abstract}
:
This paper presents a new expression for the capacitance model based on ligament efficiency. The parallel plate capacitance is the first component derived based on the percentage of ligament efficiency and the second component which is fringing field capacitance is the combination of both existing Yang's fringing field developed by the perforations and Meji's capacitance model developed for fringing field due to sidewalls of parallel plates. The proposed model is the combination of three components and enhances accuracy to estimate the total capacitance. The proposed model contributes up to $10 \%$ and $2 \%$ of the fringing field in upstate capacitance and downstate conditions. The proposed capacitance model is validated by designing in FEM tool to obtain the deviation between the results and respective deviation is expressed as the percentage of error which results from $0.013 \%$ to $2.10 \%$ in up and downstate conditions. The error percentages are analysed for different ligament efficiencies to obtain the optimum value to enhance the performance of the switch.
\end{abstract}

Keywords: Parallel plate capacitance, FEM tool, fringing Field, ligament efficiency, perforations 


\title{
PRECIPITATION AND OTHER PROPAGATION WEAKNESSES IMPACTS AT MICROWAVE AND MILLIMETRE WAVE GROUPS: A SCALED DOWN STUDY

\author{
${ }^{1}$ KOTAMRAJU S. K, 2KORADA, C. S. K.
}

Department of ECE, Koneru Lakshmaiah Education Foundation, Vaddeswaram, A.P., India522502.E-mail ID:kksarat@kluniversity.in

\begin{abstract}
:
The current past has seen an exciting addition in the usage of satellites for the applications like navigation, entertainment, media transmission, remote sensing, mobile communications, weather forecasting, defence purposes. These applications are allocated in the microwave and millimetre wave groups, which offer higher data transfer probability in lesser time and utilize very small antennas and gadgets by guaranteeing secured and effective communications. In any case, past the $10 \mathrm{GHz}$ scope of frequencies these applications are by and large exposed to signal losses because of different environmental boundaries like parameters like rain, clouds, fog, hail ice and other applicable phenomena. The fundamental factor for the signal corruption is the precipitation. The attenuation caused by rain increases with frequency, as there is expanded absorption of the RF energy at higher frequencies because of water drops present along the way of the transmission; thus, the signal attenuation is more in higher- frequency groups. Different elements that induce misfortunes in the signals are the mists, gases present in the lower climate and the various layers in the environment that cause scintillation and the system losses and cable losses. This overview article abbreviates all results identified with propagation impairments and attenuation aspects at microwave and millimetre wave frequencies covering the investigations of different researchers in most recent thirty years. Also, not many of the models created by different researchers were recorded alongside model boundaries which are useful for the propagation engineers and other people who are keen on this specialization.
\end{abstract}

Index terms: Propagation impairments $\cdot$ Rain attenuation $\cdot$ Cloud attenuation $\cdot$ Earthspace paths $\cdot$ Microwave and millimetre frequencies 


\title{
COMBINATION OF PHASED ARRAY ANTENNA FOR SIDE FLAP LEVEL DECREASE UTILIZING THE DIFFERENTIAL DEVELOPMENT ALGORITHM
}

\author{
${ }^{1}$ RAVI TEJ ${ }^{2}$ KORADA, C. S. K. ${ }^{3}$ KOTAMRAJU, S. K. \\ ${ }^{1}$ Student, Department of ECE, Koneru Lakshmaiah Education Foundation, Vaddeswaram, A.P., \\ India-522502.E-mail ID: ravi.tej489@gmail.com \\ ${ }^{2}$ Professor, Department of ECE, Koneru Lakshmaiah Education Foundation, Vaddeswaram, \\ A.P., India-522502.E-mail ID:kavya@kluniversity.in \\ ${ }^{3}$ Professor, Department of ECE, Koneru Lakshmaiah Education Foundation, Vaddeswaram, \\ A.P., India-522502.E-mail ID:kksarat@kluniversity.in
}

\begin{abstract}
:
In this paper we synthesis an array comprising of consistently excited unequally separated components for diminishing SLLs. A globally optimal solution for the array components phase and position is controlled by utilizing a differential transformative algorithm for a given radiation design. Decreased side- lobe levels are seen with position- phase combination. The acquired outcomes are compared and the conceivable two cases i.e., uniform amplitude equal phase (variable position-control) and uniform amplitude unequal phase (variable position control). The obtained results justify that the position- phase synthesis technique isn't just lessens the side- lobe levels to $(-25.28) \mathrm{dB}$ but also preserves the benefits of both variable position-controlled union and variable phase controlled synthesis with a base number of array components.
\end{abstract}

Index terms: Phased array $\cdot$ Uniform excitation $\cdot$ Side lobe level $\cdot$ Equal phase $\cdot$ Unequal spacing 


\title{
PLAN AND ANALYSIS OF CAPACITIVE SHUNT RF MEMS SWITCH FOR RECONFIGURABLE ANTENNA
}

\author{
${ }^{1}$ C.LEELA MOHAN ${ }^{2}$ KORADA, C. S. K. ${ }^{3}$ KOTAMRAJU, S. K. \\ ${ }^{1}$ Research Scholar, Department of ECE, Koneru Lakshmaiah Education Foundation, \\ Vaddeswaram, A.P., India-522502.E-mail ID: \\ ${ }^{2}$ Professor, Department of ECE, Koneru Lakshmaiah Education Foundation, Vaddeswaram, \\ A.P., India-522502.E-mail ID:kavya@kluniversity.in \\ ${ }^{3}$ Professor, Department of ECE, Koneru Lakshmaiah Education Foundation, Vaddeswaram, \\ A.P., India-522502.E-mail ID:kksarat@kluniversity.in
}

\section{ABSTRACT:}

This paper presents design and the performance investigation of capacitive shunt RF MEMS Switch. Here, the proposed Rf MEMS Switch shows the low pull in voltage for example $11.97 \mathrm{~V}$ and performed at $38 \mathrm{GHz}$ with high isolation. To improve the performance of receiving wire characteristics micro strip feeding strategy and co-planar-waveguide transmission feeding are used in this design process. The impedance coordinating of $50 \omega$ in the antenna is relies upon the width of the feed line can be seen through simulated smith graph by utilizing the Ansoft HFSS simulator. The micro strip patch antenna shows a Return loss is $-12.4264 \mathrm{~dB}$ and displays resonance at 38 $\mathrm{GHz}$ and the band width frequency from 37 to $39 \mathrm{GHz}$. After analyzing the performance of the antenna is coordinated with RF MEMS capacitive shunt switch through co-planar wave direct transmission feed line procedure. By coordinating with switch there is a frequency move OF 1 $\mathrm{GHz}$ toward directly from $38 \mathrm{GHz}$ in antenna. The resonance is happened at $39 \mathrm{GHz}$ with bandwidth frequency between range of 38 to $40 \mathrm{GHz}$. The antenna displays return loss is $-27.2666 \mathrm{~dB}$ at working frequency $39 \mathrm{GHz}$ with RF MEMS switch and total gain of the antenna at $38 \mathrm{GHz}$ with angle of $\phi=90^{\circ}$ is $5.6671 \mathrm{~dB}$. Consequently the performance of the antenna is expanded by coordinating with RF MEMS switch. These sort of reconfigurable antennas are utilized in high frequency applications at frequency scope of Ka-band and in wireless correspondence applications and satellite communication.

Index terms: RF MEMS switches $\cdot$ Pull-in voltage $\cdot$ Switching time $\cdot$ Isolation $\cdot$ Return loss $\cdot$ Insertion loss 


\title{
HYBRID BEAM DIRECTING OF KA-BAND ARRAY-FED REFLECTOR ANTENNA FOR SATELLITE COMMUNICATION LINKS
}

\author{
${ }^{1}$ SSS KALYAN ${ }^{2}$ KORADA, C. S. K. ${ }^{3}$ KOTAMRAJU, S. K. \\ ${ }^{1}$ Assistant Professor, Department of ECE, Koneru Lakshmaiah Education Foundation, \\ Vaddeswaram, A.P., India-522502.E-mail ID:ssskalyan@kluniversity.in \\ ${ }^{2}$ Professor, Department of ECE, Koneru Lakshmaiah Education Foundation, Vaddeswaram, \\ A.P., India-522502.E-mail ID:kavya@kluniversity.in \\ ${ }^{3}$ Professor, Department of ECE, Koneru Lakshmaiah Education Foundation, Vaddeswaram, \\ A.P., India-522502.E-mail ID:kksarat@ kluniversity.in
}

\begin{abstract}
:
In this paper we present the plan and investigation of Ka band array took care of reflector antenna for satellite correspondence links. The configuration comprises of bended reflector geometry of $0.8 \mathrm{~m}$ which is taken care of with five component linear array antenna working at $20.2 \mathrm{GHz}$ (Ka band). As satellite applications request beam-steering with higher gain and limited beam, we utilize and analyze electromechanical beam controlling, for example electronic and mechanical controlling (Hybrid beam directing), through exhibit took care of reflectors. To guarantee good scanning capabilities the feed exhibit is arranged in horizontal just as in vertical directions. A2-D examining of \pm 60 is accomplished when the feed component is set horizontally in central plane though \pm 3 o when the feed component is put vertically in focal plane with the proposed array fed reflector. The proposed antenna keeps up a consistent performance all through the scanning range which helps vehicular tracking and numerous other satellite applications.
\end{abstract}

Index terms: Array-fed reflector; linear array antenna; Hybrid Beam Steering; Ka band satellite links. 

Engineering and Management Research

A Peer Revieved Open Access International Journal

\title{
DESIGN AND ANALYSIS OF CIRCULAR MONOPOLE ANTENNA FOR WIRELESS APPLICATIONS
}

\author{
B.T.P. Madhav ${ }^{1}$, K. Srilatha ${ }^{1}$ \\ ${ }^{1}$ Department of ECE, Koneru Lakshmaiah Education Foundation, Vaddeswaram, A.P., India- \\ 522502 \\ E-mail: btpmadhav@kluniversity.in
}

\begin{abstract}
:
This paper proposes the design and analysis of circular monopole antenna with concentric circles and star patch with etched two concentric circles. FR4 substrate with dielectric constant of 4.4 was used. The proposed design gives return loss of -25.07 and gain of 4.73 at $2.1 \mathrm{GHz}$ operating frequency. The designed antenna is suitable for wireless applications like PCS, UMTS and ISM. The simulated and measured results are in good agreement.
\end{abstract}

Keywords: Concentric circles, PCS, Return loss, UMTS 

Engineering and Management Research

A Peer Revieved Open Access International Journal

\title{
ENHANCEMENT OF GAIN USING HYBRID RECONFIGURABLE TRAPEZOIDAL ANTENNA WITH FSS
}

\author{
B.T.P. Madhav ${ }^{1}$, K. Srilatha ${ }^{1}$ \\ ${ }^{1}$ Department of ECE, Koneru Lakshmaiah Education Foundation, Vaddeswaram, A.P., India- \\ 522502 \\ E-mail: btpmadhav@kluniversity.in
}

\begin{abstract}
:
A trapezoidal slotted FSS based notch band antenna is designed with frequency and pattern reconfigurability. The proposed antenna is fabricated on FR4 material with loss tangent 0.025 and permittivity 4.4. The proposed antenna is resonating from 0.9 to $3.8 \mathrm{GHz}$, which covers all modern communication applications. The notch is providing blockage of frequency from 1.6-2.2 GHz. PIN diodes are used for fine-tuning from 1.5 to $3.5 \mathrm{GHz}$ with switching and-30 to +30 tilt in pattern also. FSS structure beneath the antenna is giving gain more than $7.2 \mathrm{~dB}$ and developing this model for future high gain applications.
\end{abstract}

Keywords: Frequency Selective surface (FSS), Gain, Pattern Reconfigurability 

Engineering and Management Research

A Peer Revieved Open Access International Journal

DESIGN AND ANALYSIS OF CPW-FED OCTAGON SHAPED RING SLOT MONOPOLE ANTENNA

\author{
B.T.P. Madhav¹, B. Anil Babu1 ${ }^{1}$ \\ ${ }^{1}$ Department of ECE, Koneru Lakshmaiah Education Foundation, Vaddeswaram, A.P., India- \\ 522502 \\ E-mail: btpmadhav@kluniversity.in
}

\begin{abstract}
:
The design of a octagonal ring-shaped antenna with CPW and Micro strip feed is proposed for ultra wideband applications. Instead of a traditional ring micro strip antenna, the radiating area of the octagonal ring antenna used is asymmetrical. The ground plane is adjusted with two rectangular slots, where as the radiator and the ground plane are on the same plane using the available space around the radiator. The proposed antenna is simulated by using Ansys HFSS 18.0. The measured result gives a balanced agreement with the simulation results. The designed antenna with dimensions 30x30x1.6 mm $\mathrm{mm}^{3}$ provides good radiation pattern characteristics at $3.2 \mathrm{GHz}, 15 \mathrm{GHz}$ and $18 \mathrm{GHz}$ which is suitable for $\mathrm{S}$ and $\mathrm{Ku}$ band applications.
\end{abstract}

Keywords: Monopole antenna, CPW and Micro strip feed, $S$ and Ku bands 


\title{
International Journal for Innovative
} Engineering and Management Research

A Peer Revieved Open Access International Journal

\section{A TRIPLE BAND FLOWER SHAPED MICROSTRIP ANTENNA WITH CIRCULAR RING LOADING}

\author{
B.T.P. Madhav ${ }^{1}$, B. Anil Babu' ${ }^{1}$ \\ ${ }^{1}$ Department of ECE, Koneru Lakshmaiah Education Foundation, Vaddeswaram, A.P., India- \\ 522502 \\ E-mail: btpmadhav@kluniversity.in
}

\begin{abstract}
:
In this paper, design and analysis of triple band micro strip antenna is proposed for various wireless communication applications. The deigned antenna consists of circular ring loaded flower shaped antenna having a circular slot in the centre of the patch. The centre slot in the middle of the patch is responsible for improving the reflection coefficient of the antenna. The antenna gives triple band characteristics with resonating frequencies at $2.52 \mathrm{GHz} / 4.58 \mathrm{GHz} / 7.2 \mathrm{GHz}$ with bandwidth of $0.25 \mathrm{GHz}, 0.57 \mathrm{GHz}$ and $0.71 \mathrm{GHz}$ respectively. The antenna provides a maximum peak gain of $3.8 \mathrm{dBi}$ at $2.52 \mathrm{GHz}$ with Omni-directional and bidirectional patterns. The proposed antenna is designed on FR4 substrate having dimensions $41.8 \times 28 \times 1.6 \mathrm{~mm}^{3}$ with dielectric constant 4.4 and loss tangent 0.02 .
\end{abstract}

Keywords: Triple band, micro strip antenna, wireless applications 

Engineering and Management Research

A Peer Revieved Open Access International Journal

\title{
A CONCENTRIC RING HEART SHAPED MONOPOLE ANTENNA FOR WIMAX WIRELESS APPLICATIONS
}

\author{
${ }^{1}$ G B G Tilak, ${ }^{2}$ Sarat K Kotamraju, ${ }^{3}$ B T P Madhav, ${ }^{4}$ Ch Sri Kavya, \\ ${ }^{5}$ M. Venkateswara Rao \\ Department of ECE, Koneru Lakshmaiah Education Foundation, Vaddeswaram, A.P., India- \\ 522502 kksarat@kluniversity.in
}

\begin{abstract}
:
This article introduces a shattered heart-shaped monopoly antenna. The implementation of the WiMAX band has been Chosen as an goal. To obtain the appropriate band, the suggested antenna design is carried out in various iterations. In the band from $3.2 \mathrm{GHz}$ to $4.1 \mathrm{GHz}$, the proposed broken heart-shaped antenna works. In the final version, the heart-shaped double slotted ring resonator (SRR) with two distinct radius is shown to increase bandwidth $(900 \mathrm{MHz})$ and gain $(3 \mathrm{~dB})$. In this design, other parameters such as VSWR (almost $<2$ at WiMAX band), impedance (both real and imaginary), efficiency have been found, which is almost 89 percent. Therefore, the suggested broken heart-shaped monopole antenna operating on the WiMAX band shows the moderate gain, omnidirectional pattern of size reduction, high performance, etc. that are significant in the WIMAX application band.
\end{abstract}

Index Terms: Broken heart shaped antenna, WiMAX, Slotted ring resonator (SRR), VSWR. 
RASPBERRY PI BASED PERSONAL ASSISTANCE AND ARTIFICIAL INTELLIGENCE (AI) DISABLED PEOPLE

\title{
${ }^{1}$ Sarat Kumar, ${ }^{2}$ P. Kanakaraja , ${ }^{3}$ K. Ch. Sri Kayva, ${ }^{4}$ NLSP Sairam, N.ch. Hemanth, E. Saichand, M. Tejaswi, L.Sri Naga Teja
}

${ }^{1}$ Professor Department of ECE, Koneru Lakshmaiah Education Foundation, Vaddeswaram, A.P., India-522502 kksarat@kluniversity.in

${ }^{2}$ Assistant Professor, Department of ECE, Koneru Lakshmaiah Education Foundation, Vaddeswaram, A.P., India-522502 pamarthikanakaraja407@gmail.com

${ }^{3}$ Professor, Department of ECE, Koneru Lakshmaiah Education Foundation, Vaddeswaram, A.P., India-522502 kavya@kluniversity.in

${ }^{4}$ Assistant Professor, Department of ECE, Koneru Lakshmaiah Education Foundation, Vaddeswaram, A.P., India-522502 sai.nandipalli@gmail.com

\begin{abstract}
:
Today, in society, the Internet of Things ( IoT) is becoming very important. IoT is a network in which all of the physical objects (or) items linked to the internet share data from one computer to another and benefit humans as well. The main aim of this project is to use speech to monitor home appliances. Amazon Alexa, which is interlinked with the raspberry pi module, performs speech recognition in this way. In a system that is linked to raspberry pi, electrical appliances such as fans, lamps, refrigerators, etc. are incorporated to monitor the home appliances that are to be carried out by user commands that are easy to communicate with humans and computers. In society, there are many technologies for controlling home appliances, but we are implementing raspberry pi and node MCU in our project, which have been related to If This Then That (IFTTT) and Adafruit to control home appliances. We've gained command over home appliances.
\end{abstract}

Key Words: Home automation, Voice Recognition, Raspberry Pi, Relays, Amazon Alexa, Adafruit, IFTTT,. 
MODIFICATION OF PARAMETERS OF THE ITU-R TO ENHANCE THE ESTIMATION OF TROPOSPHERIC SCINTILLATION FOR TROPICAL AREAS

\author{
${ }^{1}$ John Philip B, ${ }^{2}$ Sarat K Kotamraju, ${ }^{3}$ Ch Sri Kavya \\ ${ }^{1}$ Assistant Professor, Department of ECE, Koneru Lakshmaiah Education Foundation, \\ Vaddeswaram, A.P., India-522502 bjp2klu@gmail.com \\ ${ }^{2}$ Professor, Department of ECE, Koneru Lakshmaiah Education Foundation, Vaddeswaram, \\ A.P., India-522502 kksarat@kluniversity.in \\ ${ }^{3}$ Professor, Department of ECE, Koneru Lakshmaiah Education Foundation, Vaddeswaram, \\ A.P., India-522502 kavya@kluniversity.in
}

\begin{abstract}
:
The rapid fluctuation of the received signal is scintillation. The difference in the refractivity composition of the profile of the atmosphere causes it. In low fade margin connections and at low elevation angles, this phenomenon contributes to the signal degradation and is important. For a system designer to develop an efficient system for the satellite communication link, evaluating the scintillation intensity and its statistics is therefore vital. The methodology for extracting the scintillation strength from the raw beacon signal and comparing the ITU-R scintillation prediction model with the measured results to test its acceptability over the tropical area is presented in this paper. The scintillation fade is overestimated by the ITU-R model as compared with calculated performance. The time percentage factor parameters are then adjusted to achieve a close match of the calculated effects.
\end{abstract}

Keywords: Rain Attenuation, Satellite Communications, Scintillation, Tropical Region. 


\title{
ESTIMATION OF PROPAGATION IMPAIRMENTS AND MELTING LAYER IN THE SOUTHERN PART OF INDIAN SUBCONTINENT
}

\section{${ }^{1}$ Krishna Reddy Maddikera, ${ }^{2}$ Sarat K Kotamraju, ${ }^{3}$ K. Ch. Sri Kavya, ${ }^{4}$ S. S. S Kalyan, ${ }^{5}$ Bala Gangadhar Tilak Gande}

${ }^{1}$ Research Scholar, Department of ECE, Koneru Lakshmaiah Education Foundation, Vaddeswaram, A.P., India-522502 krishnareddymaddikeras@gmail.com

${ }^{2}$ Professor, Department of ECE, Koneru Lakshmaiah Education Foundation, Vaddeswaram, A.P., India-522502 kksarat@kluniversity.in

${ }^{3}$ Professor, Department of ECE, Koneru Lakshmaiah Education Foundation, Vaddeswaram, A.P., India-522502 kavya@kluniversity.in

${ }^{4}$ Assistant Professor, Department of ECE, Koneru Lakshmaiah Education Foundation, Vaddeswaram, A.P., India-522502 ssskalyan@kluniversity.in

${ }^{5}$ Research Scholar, Department of ECE, Koneru Lakshmaiah Education Foundation, Vaddeswaram, A.P., India-522502 balagangadhar@kluniversity.in

\begin{abstract}
:
In this report, Micro Rain Radar (MRR) estimates of melting layer height in the coastal area of Andhra Pradesh are provided. Micro Rain Radar is mounted in Andhra Pradesh , India, at K L University $\left(16.44^{\circ} \mathrm{N}, 80.62^{\circ} \mathrm{E}\right)$. The melting layer height is determined by using the recordings from the Micro Rain Radar of the measured rain rate (RR) and fall velocity (W). The approximate melting layer height is compared with Machilipatnam radio probe data $\left(16.19^{\circ} \mathrm{N}, 81.36^{\circ} \mathrm{E}\right)$ separated at a distance of $70 \mathrm{Km}$, obtained from the website of the University of Wyoming. As the measurement of the melting layer height using MRR matches with the Radiosonde results, the estimated result appears to be more accurate.
\end{abstract}

Index Terms: Micro Rain Radar, Melting Layer, Radiosonde, Fall Velocity, Rain Rate. 


\title{
Design \& Analysis of Multi-Layer Edge Feed Stacked Patch Antenna for S-Band Applications
}

\author{
${ }^{1}$ I. Govardhani, ${ }^{2}$ M. Venkata Narayana, ${ }^{3}$ A.K. Chaitanya and ${ }^{4}$ K. Rajkamal \\ 1,2 Professor, Dept of ECE, KLEF, Vaddeswaram, Guntur, A.P, India \\ ${ }^{3,4}$ Research Scholar, Dept of ECE, KLEF, Vaddeswaram, Guntur, A.P, India \\ E-Mail ID: govardhanee_ec@kluniversity.in
}

\begin{abstract}
:
Micro strip patch antennas get more and more important in these days. The easy of construction and the suitability for integration with microwave integrated circuits are two more of their numerous advantages. Additionally, the simple structures make this type of antennas suitable for low cost manufacturing. And this is also one key feature of micro strip patch antennas are used in mobile communications applications. We are having mainly two problems with the patch antennas they are size reduction and bandwidth enhancement; these are two major considerations for these antennas. The purpose of this paper is to design a multi band micro strip patch antenna and to compare with the former patch antennas and then proposed the better one. In this paper we have designed the stacked patch antenna. Here we are getting the return loss at muliti band operating frequencies. The antenna is simulated by using the hfss software and we are getting the return loss $-27.9 \mathrm{db}$ at $2.7 \mathrm{GHz}$ and we are getting the gain up to $4 \mathrm{~dB}$.
\end{abstract}

Keywords - Microstrip patch antenna, stacked patch antenna, Edge feed S band applications. 


\title{
Rain Rate-Radar Reflectivity Relationship for Drop Size Distribution and Rain Attenuation Calculation of Ku Band Signals
}

\section{Govardhani. Immadi , Sarat K Kotamraju ${ }^{2}$, Habibulla Khan ${ }^{3}$,M. Venkata Narayana $^{4}$}

\author{
1,2, 3,4 Professor, Dept of ECE, KLEF, Vaddeswaram, Guntur, A.P, India \\ E-Mail ID: govardhanee_ec@kluniversity.in
}

\begin{abstract}
:
With the increased demand for long distance Tele communication day by day, satellite communication system was developed. Satellite communications utilize L, C, Ku and $\mathrm{Ka}$ bands of frequency to fulfil all the requirements. Utilization of higher frequencies causes severe attenuation due to rain. Rain attenuation is noticeable for frequencies above $10 \mathrm{ghz}$. Amount of attenuation depends on whether the operating wave length is comparable with rain drop diameter or not. In this paper the main focus is on drop size distribution using empirical methods, especially Marshall and Palmer distributions. Empirical methods deal with power law relation between the rain $\operatorname{rate}(\mathrm{mm} / \mathrm{h})$ and radar reflectivity $(\mathrm{dBs})$. Finally, it is discussed about the rain rate variation, radar reflectivity, drop size distribution, that is made for two rain events at K L University, Vijayawada on 4 September 2013 and on 18 August 2013.
\end{abstract}

Keywords-Rain rate, Radar Reflectivity, Drop size distribution 
DESIGN OF UHF ANTENNA FOR WIRELESS APPLICATIONS USING DEFECTIVE GROUND

\author{
${ }^{1}$ Govardhani Immadi, ${ }^{2}$ M. Venkata Narayana, ${ }^{3}$ Ch. S. S. Reddy, ${ }^{4}$ P. ${ }^{5}$ J. Sivani \\ and ${ }^{6}$ P. V. S. Anil Kumar \\ ${ }^{1,2}$ Professor, Dept of ECE, KLEF, Vaddeswaram, Guntur, A.P, India \\ E-Mail ID: govardhanee_ec@kluniversity.in
}

\begin{abstract}
:
In this article, we describe a novel type of defective ground surface (DGS) microstrip antenna which has higher gain, multi resonant frequency with compact size. It consists of hexagonal shaped patch with small volume and the ground plane is cut into six triangular slots just below the corners of the hexagonal patch. Antenna is circularly polarized one with gain $3.6 \mathrm{dBi}$. The antenna shows multi resonant frequency. The proposed antenna is simple in structure compared with coplanar parasitic patch antennas. It is mostly suitable for wireless communications. slots is placed for improving the bandwidth.
\end{abstract}

Keyword: defective ground structure, slots, returns loss, gain, radiation pattern. 


\title{
ESTIMATION OF EFFECT OF TROPOSPHERE RAIN ON RADIO LINK IN TROPICAL ENVIRONMENT
}

\author{
Govardhani Immadi and M. Venkata Narayana, Y. Suraj, N. M. V. L Nara \\ Simha Rao, P. S. V. S. Naveen Chowdary and M. Emmanuel Raju \\ Department of Electronics and Communication Engineering, KL University, Vaddeswaram, \\ Guntur, Andhra Pradesh, India \\ E-Mail: govardhanee_ec@kluniversity.in
}

\begin{abstract}
:
Rain has deleterious impact on satellite signal propagation above $\mathrm{Ku}$-band due to scattering and absorption. Numerous Empirical and Non-empirical models are evolved based on measured statistics to estimate the rain attenuation. The day wise, monthly and yearly analysis for 3 years of data is performed in Vaddeswaram. Of the available models, for the tropical region, ITU-R model which uses bulk recorded database clearly underestimates the value. In this paper different attenuation models like ITU-R, RH, SAM and Moupfouma are studied and the results are compared with measured values and analysed to determine the suitable model for one of the tropical regions Vaddeswaram, A.P. It is observed that the average attenuation is around $13.5 \mathrm{~dB}$ in a year and Moupfouma model is best suited for this region.
\end{abstract}

Keywords: rain attenuation, effective path length, rain rate exceedance, beacon data. 


\title{
2x2 Microstrip Patch Antenna Using Corporate Feed
}

\author{
${ }^{1}$ M. Venkata Narayana, ${ }^{2}$ I. Govardhani, ${ }^{3}$ K. Rajkamal and ${ }^{4}$ A. K. Chaitanya \\ ${ }^{1,2}$ Professor, Dept of ECE, KLEF, Vaddeswaram, Guntur, A.P, India \\ ${ }^{3,4}$ Research Scholar, Dept of ECE, KLEF, Vaddeswaram, Guntur, A.P, India \\ E-Mail ID: govardhanee_ec@kluniversity.in
}

\begin{abstract}
:
There are many applications in wireless communication that involve more than one distinct band of frequencies. The present model is a square patch array of $2 \times 2$ i.e. 4 elements arranged on the FR4 epoxy substrate material. The present model is useful at the $2.75 \mathrm{GHz}$ we are getting the return loss up to $15 \mathrm{~dB}$ and the gain of the array antenna is $17 \mathrm{~dB}$ All the output parameters, radiation patterns and vswr patterns are presented. Her we are using the corporate feeding for the array elements; the antenna is simulated by using the HFSS software
\end{abstract}

Keywords - Micro strip, patch antenna, array antenna, corporate feeding 


\title{
Design \& Analysis of Electrically Small Top Loaded Antennas for GPS Applications
}

\section{Venkata Narayana, Govardhani. Imamdi, Habibulla Khan, P. V. N. Lakshmi Durga, N. Sirisha, R. Praveen Kumar Reddy and Y. Chandan Krishna}

Department of Electronics and Communication Engineering, KL University, Vaddeswaram, Andhra Pradesh, India

E-Mail: mvn@kluniversity.in

\begin{abstract}
:
The design of small high frequency antennas continues to be a challenge while maintaining required bandwidth and low-quality factor. Numerous methods are proposed to reduce the quality factor for small antennas. According to Wheeler and Chu limitation on quality factor the design should be in a way that the fields inside the spherical volume should be diminished. Generally, for small antennas reactance will be very high. In this project we want to implement top loading approach to decrease $\mathrm{Q}$ value and tune the antenna at desired frequency. In this approach we used top loading for antenna in order to reduce its reactance. The top loading techniques which we implemented in this paper are loading using inductive loading, cap hat loading, and umbrella loading for helical antennas using ANSYS HFSS 13.0. The frequency of this antenna is designed at $1575.42 \mathrm{MHz}$ which is operating frequency for GPS. GPS is used for tracking purposes. Q factor is greatly reduced by using these design approaches.
\end{abstract}

Keywords: small antennas, $Q$ factor, top loading, helical antenna. 


\title{
Analysis of Small Antenna with Slotted Meander-Line Resonator For Wireless Applications
}

\section{Venkata Narayana, Govardhani Immadi, Habibulla Khan, K. Venkata Karthik, G. Hari Babu, P.V. S. Pruthvi Kumar Reddy and V. Sahithi}

Department of Electronics and Communication Engineering, KL University, Vaddeswaram,

Andhra Pradesh, India

E-Mail: mvn@kluniversity.in

\begin{abstract}
:
Day by day with the increasing of demand for long distance communication resulting in requirement of many antenna elements on a single structure and if we do so there is a problem of coupling between those elements which reduces the antenna efficiency. So a new methodology to improve the isolation in micro strip patch antenna arrays is described through the implementation of a slotted meander-line resonator (SMLR) which is creating defect in the microstrip structure. The resonator is designed to suppress the surface current between the two patch antennas coupled along Hplane and operating at a frequency of $5 \mathrm{GHz}$ which is used in microwave links and airborne RADAR applications. The configuration has been designed, simulated and validated experimentally.
\end{abstract}

Keywords: antenna arrays, isolation, coupling, antenna efficiency, surface current meander lines, resonators. 


\title{
Analysis of Microstrip Circular Patch Antenna Array
}

\section{Venkata Narayana, Govardhani. immadi, Harika Muppa, Mahesh Nagisetty, Harsha Vardhan Reddy Konda}

Department of Electronics and Communication Engineering, KL University, Vaddeswaram,

Andhra Pradesh, India

E-Mail: mvn@kluniversity.in

\begin{abstract}
:
This paper analyses the design of an antenna arrays with circle patch which is intended with a frequency of $2.5 \mathrm{GHz}$, and its main application in wireless communications. This array is used for obtaining high gain. From a basic antenna having circular patch, some new elements are introduced to get linear array. These linear arrays are used to attain high gains. For the antenna shown here, the achieved gain is $5.21 \mathrm{~dB}$, return loss (VSWR) is $\leq-10 \mathrm{~dB}$. The antenna array was agitated by uniform micro strip line feed method. An analysis was done considering $1 \mathrm{x} 4$ array. It is established that gain increases at a cost of B.W.
\end{abstract}

Keywords: antenna arrays, microstrip, patch antenna. 


\title{
Non-Destructive Characterization of CFRP Composite using Non Stationary Thermal Wave Imaging
}

\author{
G.V.P. Chandra Sekhar Yadav ${ }^{1}$, V.S. Ghali ${ }^{1}$ \\ ${ }^{1}$ Infrared Imaging Center, Department of ECE, Koneru Lakshmaiah Education Foundation, \\ Green Fields, Vaddeswaram, A.P., India-522502. \\ E-mail: gvs0raos@gmail.com.
}

\begin{abstract}
:
The Non-destructive testing plays a vital role in industrial and biomedical applications. Nonstationary stimulation based active Infrared thermography is an emerging area of interest in subsurface defect detection and visualization. In present article, frequency modulated thermal wave imaging is employed on a numerical simulation to detect defects of CFRP specimen and applied various post processing techniques such as FFT phase, Pulse compression, principal component analysis and random projection transform for better defect detection. Defect Signal to noise ratios considered as merit of analysis.
\end{abstract}

Index Terms - Frequency modulated thermal wave imaging, Signal to noise ratio, Nonstationary thermal wave imaging, CFRP, Spectral reshaping, Pulse compression, PCA, Random projection transform. 


\title{
Black-hole Attacks in AODV Routing Protocol for Mobile Ad- hoc Networks
}

\author{
D. Kondamacharyulu ${ }^{1}$, N. Srinivasulu ${ }^{2}$ \\ ${ }^{1}$ Department of ECE, Koneru Lakshmaiah Education Foundation, Green Fields, \\ Vaddeswaram, A.P., India-522502. \\ ${ }^{2}$ Department of ECM, Koneru Lakshmaiah Education Foundation, \\ Green Fields, Vaddeswaram, A.P., India-522502.
}

\begin{abstract}
:
A black hole attack is a severe attack that can be easily employed against routing in mobile adhoc networks. A black hole is a malicious node that falsely replies for any route requests without having active route to specified destination and drops all the receiving packets. If these malicious nodes work together as a group, then the damage will be very serious. This type of attack is called cooperative black hole attack. In this paper, we are implementing Black hole attack considering the routing protocol: Ad-hoc On Demand Vector Routing Protocol (AODV) evaluate the network performance metrics like throughput, First route failure lifetime, Packet-Delivery Ratio, Average end-end Delay, Drop rate. The Experiment show that (1) Implementation of AODV for MANET without Black hole attacks (2) AODV for MANET suffers from Cooperative Black hole attack (3) Comparison of AODV without Black hole attacks and with Black hole attacks in terms of Network Performance Metrics.
\end{abstract}

Index Terms - MANET, Black hole attack, Network Performance, Security, Throughput, Packet loss and Packet Delivery Ratio 


\title{
Performance Metrics between Routing Protocols for MANETS
}

\author{
D. Yamini ${ }^{1}$, N. Srinivasulu ${ }^{2}$ \\ ${ }^{1}$ Department of ECM, Koneru Lakshmaiah Education Foundation, Green Fields, \\ Vaddeswaram, A.P., India-522502. \\ ${ }^{2}$ Department of ECM, Koneru Lakshmaiah Education Foundation, \\ Green Fields, Vaddeswaram, A.P., India-522502.
}

\begin{abstract}
:
In wireless communication networks Ad-hoc networks are plays dominant role, Mobile Ad-hoc network (MANET) is a collection of wireless mobile nodes that dynamically form a network temporarily without any central administration. The primary objective of this research work is to study and investigate the performance of Dynamic source routing (DSR) protocol and Energy efficient routing protocols like MBCR and MMBCR. Energy efficient routing is one of the important design criterions for MANET since mobile nodes are battery powered with limited capacity and which cannot be recharged whenever needed. So the MANET routing is challenged by power and bandwidth constraints. We use CBR based Traffic models to analyses the performance of routing protocols based on parameters of Packet Delivery Ratio, Average end to end Delay, Energy Consumption, Node Analysis, Network Lifetime and through put. We have used NS-2 Simulator for simulation.
\end{abstract}

Index Terms - Ad-hoc Network, Avg end to end delay, Energy efficiency, Network Lifetime, Packet Delivery Ratio. 


\title{
Visualization of Performance Metrics between Routing Protocols for MANETS
}

\author{
Kumar Sai $^{1}$, N. Srinivasulu ${ }^{2}$ \\ ${ }^{1}$ Department of ECM, Koneru Lakshmaiah Education Foundation, Green Fields, \\ Vaddeswaram, A.P., India-522502. \\ ${ }^{2}$ Department of ECM, Koneru Lakshmaiah Education Foundation, \\ Green Fields, Vaddeswaram, A.P., India-522502.
}

\begin{abstract}
:
In wireless communication networks Ad-hoc networks are plays dominant role, Mobile Ad-hoc network (MANET) is a collection of wireless mobile nodes that dynamically form a network temporarily without any central administration. The primary objective of this research work is to study and investigate the performance of Dynamic source routing (DSR) protocol and Energy efficient routing protocols like MBCR and MMBCR. Energy efficient routing is one of the important design criterions for MANET since mobile nodes are battery powered with limited capacity and which cannot be recharged whenever needed. So the MANET routing is challenged by power and bandwidth constraints. We use CBR based Traffic models to analyses the performance of routing protocols based on parameters of Packet Delivery Ratio, Average end to end Delay, Energy Consumption, Node Analysis, Network Lifetime and through put. We have used NS-2 Simulator for simulation.
\end{abstract}

Index Terms - MANET, Packet delivery ratio, Network life time, Average end to end delay, Dropped packets. 


\title{
Efficient Cell Sizing of Single Precision Floating Point ALU for DSP Applications
}

\author{
D. Siva Sai ${ }^{1}$, N. Srinivasulu ${ }^{2}$ \\ ${ }^{1}$ Department of ECE, Koneru Lakshmaiah Education Foundation, Green Fields, \\ Vaddeswaram, A.P., India-522502. \\ ${ }^{2}$ Department of ECM, Koneru Lakshmaiah Education Foundation, \\ Green Fields, Vaddeswaram, A.P., India-522502.
}

\begin{abstract}
:
Technological advancement in satellite communication and signal processing technology, high frequency of operation with low noise margin is required. So, the technological advancement has resulted in the introduction of digital technology where digital processing units had created more impact. The ALU plays a crucial role in digital technology by encrypting the data and providing more secrecy and security. Numerous ALU blocks are required in DSP units for the proper transfer of the signals. As a result, various methodologies are introduced for the design of ALU unit. The most recent history of digital technology suggests that there is a rapid growth in various technological aspects leading to compression of technologies from micro level to Nano level at a very faster rate. So various lateral thinking methodologies had come into existence for the designing process. This led to a thought of designing a most significantly used digital block of the circuitry with the help of advantageous and advanced methods. In this paper where the basic problem in the blocks like sizing can be reduced and power consumption can be explained. A righteous and well executed tool are used in the design methodology to properly regulate and monitor the basic parameters. Most preferable XILINX VIVADO is used in the process of design.
\end{abstract}

Index Terms - Digital signal processing, Single Precision, IEEE 754 PROTOCOL, SOC 


\title{
Implementation of Environment Gases Monitoring System using LoRa Gateway in Smart Cities with IoT Technology
}

\section{Syed Sahir, Yugandhar Abbina, P Gopi Krishna, Ankit Karak, Shaik Razia}

U.G. Student, Dept. of ECM, Koneru Lakshmaiah Education Foundation, Vaddeswaram, Guntur, A.P, India

U.G. Student, Dept. of ECM, Koneru Lakshmaiah Education Foundation, Vaddeswaram, Guntur, A.P., India

Associate Professor, Dept. of ECM, Koneru Lakshmaiah Education Foundation, Vaddeswaram, Guntur, A.P, India

U.G. Student, Dept. of ECM, Koneru Lakshmaiah Education Foundation, Vaddeswaram, Guntur, A.P, India

\begin{abstract}
The Paper aims in the design and implementation of toxic gases monitoring systems in the environment of smart cities using the LoRa internet of things (IoT) gateway. The prototype uses Lora WAN which as the features of low power consumption of battery, high coverage range, security and adaptability of data rates according to the environment basing on the distances of the nodes placed. The system identifies the toxic gases and sends it to the cloud and gives the notification to the end-users when it crosses the predefined value set in the application program. A GPS module is integrated in the systems that sends the exact location i,e longitude and latitude to the cloud. Using the cloud, the end user can monitor the percentage of gases present in the environment and we can see the using the goggle maps. As a proof of demonstration, the prototype is placed in different parts of the city.
\end{abstract}

Keywords: Internet of Things (IoT), Lora WAN, Smart Cities, LoPy, GPS. 


\title{
An Embedded Web Server Based Automatic Smart Home Monitoring System
}

\author{
S. Anusha, K. Sreenivasa Ravi and P. Gopi Krishna \\ M.Tech Student (ES), Department of Electronics and Computer Engineering, KL University, Vaddeswaram, Guntur, \\ A.P. Email: sulamanusha481@gmail.com \\ Professor, Department of Electronics and Computer Engineering, KL University, Vaddeswaram, Guntur, A.P. \\ Email: ravi.kavuluri@kluiversity.in \\ Asst. Professor, Department of Electronics and Computer Engineering, KL University, Vaddeswaram, Guntur, A.P. \\ Email: gopikrishna.popuri@gmail.com,
}

\begin{abstract}
Smart home is an observing, controlling and investigating service which includes Wireless transmission technology and electronic sensor innovation. It allows the client to get the full scope of services, the opportunity for continuous monitoring and controlling of home environment. An embedded web server is developed which consists of screens for home environment parameters, for example, room temperature, light intensity, LDR sensor, PIR sensor, motion detection, fire detection, smoke sensor, humidity sensor and LPG gas leakage for monitoring and controlling remotely. In this project Raspberry $\mathrm{Pi} 3 \mathrm{~B}+$ is used for monitoring, processing, controlling different sensors and communicating with embedded web server, because of its advanced features and easy of communicating through Internet of Things (IoT)..
\end{abstract}

Keywords: Internet of Things (IoT), Sensor node, Raspberry pi, Embedded Web server 


\title{
Lora System based Accident Rescue System for Future Generation
}

\author{
N.V.K. Ramesh, Puctchapurnapriya, N. Suresh, Shaik Razia, B Naresh \\ Kumar Reddy, P. Gopi Krishna \\ 1Department of ECE, Koneru Lakshmaiah Education Foundation, Vaddeswaram, Andhra Pradesh, India, \\ Department of ECM, Koneru Lakshmaiah Education Foundation, Vaddeswaram, India,
}

\begin{abstract}
Fatal crashes are increasing exponentially in India. Death rates have a high impact on any Nation. The existence of the harmed individual could be spared by providing clinical assistance at the right time. The conventional techniques are basically focused on transmitting messages using the cell arrangement. This endeavor's technique is to recognize the area of the accident region and send those directions to the nearest locations of clinical assistance. With the aid of the Bluetooth gadget the communication occurs between the commuter and the device. GPS gadget is very noteworthy for the recognizable proof of the present location. Using GPS this will eliminate the directions of the accident-prone zone. The geographic directions of the different medical clinics will be stored in the database. It is best to achieve the directions of the accidentprone region and medical clinic directions in the database, as well as to determine the distance between them. The directions of the closest available medical clinics can be seen and communication can be established with the health facility.
\end{abstract}

Key words: GPS, Wireless control, FEC, CSS, SP. 


\title{
A Vehicle-to-Vehicle Communication using can Network and ZIGBEE Technology
}

\author{
R. Pavani, P.S.G. Arunasri and P. Gopi Krishna \\ M-Tech Student (ES), Department of Electronics and Computer Engineering, KL University, \\ Vaddeswaram, Guntur, A.P. Email: pavanirontala@gmail.com \\ Assoc. Professor, Department of Electronics and Computer Engineering, KL University, \\ Vaddeswaram, Guntur, A.P. Email: arunasri_2012@kluniversity.in \\ Asst. Professor, Department of Electronics and Computer Engineering, KL University, \\ Vaddeswaram, Guntur, A.P. Email: gopikrishna.popuri@gmail.com,
}

\begin{abstract}
This project is designed for the usage of a driving assistance technology. The main intention of this operation is vehicle to vehicle and within the vehicle statistics sharing. The gadgets are primarily based on cloud to notify the driver about the basic data of nearby automobiles. Once specific space is identified by the driver, he can drive the vehicle consequently. Vehicular system is a new subject of research it goals to examine remote agents (vehicles, people, robots) to interact, collaborate and to sense the surroundings. It processes the information, disseminate the results, and more usually share resources. During this project we tend to sense the parameters equivalent to pressure, fire, gas, object detection, and temperature. Even accident detection indicator and wireless communication can be added as an advantage to the system. Most of these parameters can be shared in a vehicular network and this could be monitored through any server application.
\end{abstract}

Keywords: ARM7, CAN, Sensors. 


\title{
DM3730 Processor Hardware Debugging on Linux Platform
}

\author{
B. Madhusudhan Reddy ${ }^{1}$, Anumandla Kiran Kumar ${ }^{2}$ \\ ${ }^{1}$ Vignan Institute of Technology and Science, Deshmukhi, Hyderabad, India. \\ ${ }^{2}$ Department of ECE, Koneru Lakshmaiah Education Foundation, \\ Green Fields, Vaddeswaram, A.P., India-522502.
}

\begin{abstract}
:
DM3730 processor is fabricated on single chip Texas Instrument's advanced 45-nm technology with improved performance. ARM architecture provides best Graphics with less power consumption. DM3730 1GHz processor comes in $0.4 \mathrm{~mm}$ pitch POP (Package on Package) with memory mounted on top of the processor. Debugging at the hardware/software level is necessary for developing an efficient system. Image Processing application requires, Camera, Speakers, GSM module, keyboard, mouse, internet connection for installing required software's, hence hardware debugging is must to test and know the working of these peripherals properly. The hardware/software debugging is the need of the today's industry for proper deliver of the system. In this paper, DM3730 Processor is tested for different peripherals on Linux Platform.
\end{abstract}

Index Terms - Camera, DM3730, GS, Image 


\title{
HARDWARE PROTOTYPING FOR VIDEO AND SIGNAL PROCESSING APPLICATIONS USING DM 6437 TEXAS INSTRUMENT EVALUATION BOARD
}

\author{
B. Madhusudhan Reddy ${ }^{1}$, Anumandla Kiran Kumar ${ }^{2}$ \\ ${ }^{1}$ Vignan Institute of Technology and Science, Deshmukhi, Hyderabad, India. \\ ${ }^{2}$ Department of ECE, Koneru Lakshmaiah Education Foundation, \\ Green Fields, Vaddeswaram, A.P., India-522502.
}

\begin{abstract}
:
In real time applications, Signal and Video processing provides an interesting research area such as in medical field, surveillance, biometric, etc., This paper presents fast prototyping to develop signal and video processing application using DM 6437 Evaluation board. During the experimental research DM 6437 EVM is programmed with Matlab/Simulink model and code composure studio version 4 software. This paper presents the complete procedure to implement signal and video processing applications in real time with TMS320DM6437 Texas Instrument Evaluation board.
\end{abstract}

Index Terms - CCCSv4, DM 6437 EVM, API, CAN, I2C, USART, DVSDK, C6000, C6xCSL 


\title{
Optimization of smart vehicle ad hoc network (SVANET) communication for traffic related issues with a security
}

\author{
B. Madhusudhan Reddy ${ }^{1}$, Anumandla Kiran Kumar ${ }^{2}$ \\ ${ }^{1}$ Vignan Institute of Technology and Science, Deshmukhi, Hyderabad, India. \\ ${ }^{2}$ Department of ECE, Koneru Lakshmaiah Education Foundation, \\ Green Fields, Vaddeswaram, A.P., India-522502.
}

\begin{abstract}
:
Nowadays Vehicle Ad hoc Networks have an interesting research and application area in real time scenario. Vehicles are embedded with smart embedded sensors, processing ability and wireless communication capabilities will lead to an efficient development of road safety, comfort, information sharing and efficient control while on the road. In this paper, traffic related issues like signal jumps, expiry of RCs, Pollution status etc., can be identified and corrective actions can be initiated. This system can also be used for other applications like traffic density identification, signs of other vehicles like turnings, ambulance signs, advertisements etc. This work mainly targets to route the information from vehicle to gateway and from gateway to vehicle efficiently. In vehicle to gateway communication, firstly gateway will broadcast the signals to all surrounding vehicles. If any vehicle is detected by the gateway, then the gateway sends a command to the corresponding vehicle to send their id information. Then the gateway verifies the information of RC, Pollution status, pending challans status, and if any discrepancies are found, then the vehicle is notified. The proposed work is implemented on LPC2148 microcontroller using RFID technology.
\end{abstract}

Index Terms - RSRC, LPC 2148, NS2, RFID, Mobile nodes, SVANET 


\title{
Hardware Implementation of TLBO Algorithm for Cognitive Radio Networks
}

\author{
Anumandla Kiran Kumar ${ }^{1}$, Kiran Kumar Maddipati ${ }^{1}$, Y. Tirumala Reddy ${ }^{1}$ \\ ${ }^{1}$ Department of ECM, Koneru Lakshmaiah Education Foundation, \\ Green Fields, Vaddeswaram, A.P., India-522502.
}

\begin{abstract}
:
Teaching Learning Based Optimization (TLBO) algorithm simulate the teaching learning peculiarity of a classroom to solve multi-dimensional, linear and nonlinear problems with appreciable efficiency. In order to accelerate the execution time of software implementation, the TLBO algorithm is implemented on hardware. Then the TLBO hardware is developed as TLBO Intellectual Property and it is interfaced as a peripheral to the System on Chip platform. We compared the performance of the floating point TLBO IP is realized by solving the benchmark functions, results 183-224X times faster than the software implementation of the same algorithm. As a case study, the same TLBO IP is used to solve the spectrum allocation problem by optimizing Max-Sum-Reward (MSR) function and it results 69-78X times faster than the software implementation of the same algorithm.
\end{abstract}

Index Terms - Field Programmable Gate Array, Spectrum Allocation, Teaching Learning based Optimization 


\title{
Cloud Enabled Neural Network with Intelligent Sensor nodes for HVAC
}

\author{
Ragipati Karthik $^{1}$, K. Aravind Reddy ${ }^{1}$, R.P.V.N.N.Kumar ${ }^{1}$ \\ ${ }^{1}$ Department of ECM, Koneru Lakshmaiah Education Foundation, \\ Green Fields, Vaddeswaram, A.P., India-522502.
}

\begin{abstract}
:
HVAC (Heating, Ventilation and Air Conditioning) is the technology of indoor and vehicular environmental comfort and to control these systems. The status of building energy consumption is increasingly prominent. Indoor air pollution is 10times danger than outdoor due to incorrect functionality of heating, ventilation, and air condition system. For indoor environment quality, a novel real-time method for HVAC system operation is developed. Internet of things is used to monitor the indoor air quality by using embedded electronics, software and sensors and connectivity. This project aims to integrate air condition, ventilation and protected system on a single embedded system that alerts early warning for the unpredictable dangers. The wireless sensor nodes have limited processing power and memory. In order to embed intelligence into sensor nodes, a hybrid algorithm is proposed containing RNN (Random Neural Network) and LNP (Linear Non-linear Poisson) cascade model.
\end{abstract}

Index Terms - AIR QUALITY, HVAC, INTERNET OF THINGS. 


\title{
Energy Monitoring Using Arm 7
}

\section{Ragipati Karthik $^{1}$, Bellamkonda Jyothi ${ }^{1}$, Dronamraju Sruthi ${ }^{1}$ \\ ${ }^{1}$ Department of ECM, Koneru Lakshmaiah Education Foundation, Green Fields, Vaddeswaram, A.P., India-522502.}

\begin{abstract}
:
In this project we can introduce the power or electricity monitoring is a major challenge in electricity distribution system. Consumer deceit is a problem faced by all power corporations. Electricity Suppliers Companies are having large amount of money loss due to electricity wastage a by consumers. Electricity management is the use of electric power without paying the bill amount. Detection of electricity is very difficult and requires continuous monitoring to reduce fraud. Distributed Power utilized by consumer from electricity. In this project there is a tow subsystems they are voice control and phone control system. Using Bluetooth for controlling the devices by the phone monitoring system. And also calculating the amount of the power usage by the ARM 7 microcontroller. Phone monitoring controlling system is convenient for the user better monitoring the usage of their phones
\end{abstract}

Index Terms-ARM7, Bluetooth, Relay, LCD, Power Supply, current transportation (ct), potential transportation(pt). 


\title{
Smart Vehicle Tracking System using RFID
}

\author{
Ragipati Karthik $^{1}$, T. Harshavardhan ${ }^{1}$, M. Vinod Reddy ${ }^{1}$ \\ ${ }^{1}$ Department of ECM, Koneru Lakshmaiah Education Foundation, \\ Green Fields, Vaddeswaram, A.P., India-522502.
}

\begin{abstract}
:
A proficient vehicle tracking framework is projected and executed for the development of any vehicle following from any area at any time. The proposed arrangement takes points of interest of the two fundamental highlights in portable stage these days which are area administrations, predominantly GPS-GSM based, and essential communication administrations, principally SMS based. The utilization of RFID per user is to peruse the tag of a vehicle and the data will be sent to the framework, the highlights incorporate controlling the framework through UI. The contributions from RFID per users are persistently refreshed To Arduino for handling the information. The server gadget's primary duty is to give the careful area of the transport to the server, or the client if there should be an occurrence of SMS based inquiry from customer's gadget. Then again, customer's gadget can discover transport area either utilizing SMS administration or utilizing network access. If customer uses android mobile, user can introduce the application to follow the transport area utilizing web access. The server gadget will be put on the vehicle of enthusiasm with our application introduced inside it. It performs preferred from numerous points of view over other comparative vehicles following frameworks. The proposed framework utilized a prevalent innovation that joins a Smartphone application.
\end{abstract}

Index Terms -GPS and GSM Module, RFID TAG, Smartphone Application, Vehicle Tracking. 


\title{
IoT based forest fire detection system
}

\author{
M. Trinath Basu ${ }^{1}$, Ragipati Karthik ${ }^{1}$, J. Mahitha ${ }^{1}$, V. Lokesh Reddy ${ }^{1}$ \\ ${ }^{1}$ Department of ECM, Koneru Lakshmaiah Education Foundation, \\ Green Fields, Vaddeswaram, A.P., India-522502.
}

\begin{abstract}
:
It has been found in a survey that $80 \%$ losses caused due to fire would have been kept away from if the fire was identified promptly. Node Mcu based IoT empowered fire indicator and observing framework is the answer for this issue.In this task, we have assembled fire finder utilizing Node Mcu which is interfaced with a temperature sensor, a smoke sensor and signal. The temperature sensor detects the warmth and smoke senssor detects any smoke produced because of consuming or fire. buzzer associated with Arduino gives us an alert sign. At whatever point fire activated, it consumes protests adjacent and produces smoke. A fire caution can likewise be activated because of little smoke from candlelight or oil lights utilized as a part of a family. Likewise, at whatever point warm force is high then additionally the alert goes on. Bell or alert is killed at whatever point the temperature goes to ordinary room temperature and smoke level decreases. We have additionally interfaced LCD show to the Node Mcu board.With the assistance of IoT innovation.Node MCU fire checking serves for mechanical need and also for family unit reason. At whatever point it recognizes fire or smoke then it immediately alarms the client about the fire through the ethernet module. For this reason, we are utilizing ESP8266 which is from Arduino IDE. Likewise, the Node Mcu interfacing with LCD show is done to show the status of the framework whether the Smoke and Overheat is identified or not. What's more, Node Mcu interfacing with Ethernet module is done as such that client become more acquainted with about the predominant condition message. It insinuate the client about the fire identification. This framework is extremely helpful at whatever point the client isn't in the closeness of control focus. At whatever point a fire happens, the framework naturally faculties and alarms the client by sending an alarm to an application introduced on user's Android portable or page open through web.
\end{abstract}

Index Terms - GPS, LCD Display, GSM, Smoke Sensor, Ethernet, Radio Frequency. 


\title{
Big Data Analytics and IoT Gadgets for Tech Savvy Cities:
}

\author{
Arvind Yadav ${ }^{1}$, Ramesh Kumar Mojjada ${ }^{1}$ \\ ${ }^{1}$ Department of ECM, Koneru Lakshmaiah Education Foundation, \\ Green Fields, Vaddeswaram, A.P., India-522502.
}

\begin{abstract}
:
In towns and urban areas to make it as a smart and to address the issues of urban open and the city advancement shrewdly, the utilization of IoT gadgets, and the savvy framework is the quick and profitable source. In any case, interconnecting a large number of IoT gadgets while speaking with each other over the Web to build up a keen frame work, brings about the age of colossal measure of information, named as huge data or a big data. To incorporate IoT benefits so as to get constant city information and afterward preparing such enormous measure of information in a productive route went for setting up brilliant city is a testing assignment. In this manner, we processed and built up a smart city framework in light of IoT utilizing big data and Investigation utilizing Flume and Hive. Hive is a rank sharing center foundation device to process ordered data in Hadoop. It dwells over Hadoop to abridge Huge Information. It can be utilized for dumping movement information in Hadoop Circulated Record Framework. We utilize sensors structure including keen home sensors, vehicular systems administration, climate and water sensors, smart stopping sensors, observation objects, and so on. The total engineering and usage is proposed, which is executed utilizing Hadoop biological community in a genuine domain. The framework usage comprises of different advances that begin from information age and gathering, collecting, filtration, characterization, preprocessing, figuring and completed at basic leadership. The framework is for all purposes actualized by taking city information source to create a smart city. The work establishes the anticipated framework is effective and versatile environment.
\end{abstract}

Index Terms - IOT, Bigdata, Hadoop, Hive 


\title{
Performance Analysis of Layered Architecture to integrate Mobile Devices and Grid Computing with a Resource Scheduling Algorithm
}

\author{
Gaurav Kumar ${ }^{1}$, Ramesh Kumar Mojjada ${ }^{1}$ \\ ${ }^{1}$ Department of ECM, Koneru Lakshmaiah Education Foundation, \\ Green Fields, Vaddeswaram, A.P., India-522502.
}

\begin{abstract}
:
This paper proposes a layered system model to bridge the gap between mobile and grid computing world. The model divides the complexities in mobile grid integration, among different components of different layers of the proposed model. In this model of the system we have an efficient algorithm which addresses the problem of scheduling and disconnection. The model proposes an algorithm to address resource scheduling problem and primarily focus on intermittent disconnections problem of the mobile devices, battery power and economy. In the present era of digital technology is growing in a rapid speed. As the number of users are increasing the mobile data processing is very essential that is a very complex task. It can be done by the cloud environment for complex computations grid computing is used efficiently
\end{abstract} Index Terms -cloud computing, layer architecture, complexities 


\title{
Securing SAAS service under cloud computing based multi- tenancy systems
}

\author{
Trinad Basu ${ }^{1}$, Ramesh Kumar Mojjada ${ }^{1}$ \\ ${ }^{1}$ Department of ECM, Koneru Lakshmaiah Education Foundation, \\ Green Fields, Vaddeswaram, A.P., India-522502.
}

\begin{abstract}
:
Cloud computing technologies are being used by many who need computing resources such as software, platform and infrastructure as per their business requirements in terms of provisioning and pay for the usage as per actual consumption of the services based on the SLA signed by the user and cloud service provider. Software running on a physical machine is being provided as services to the end users. For the reasons of cost economies access to software that uses a database is being provided to multiple users. The access to the software is provided either directly or through a virtual machine. The software being provided as service uses the same database for many of the users who have requisitioned for the same. As a result, there could be encroachments by the users into the data of others. There is a need to secure the data belonging to several users while all of them access the data using the same application. In this paper an efficient method is presented for securing the data processed by software which is offered as a service to multiple users either directly or through virtual machines.
\end{abstract}

Index Terms -SaaS, Cloud Computing, Multi-tenancy, shared data services 


\title{
A transfer learning framework for traffic video using neuro- fuzzy approach
}

\author{
Trinad Basu ${ }^{1}$, Ramesh Kumar Mojjada ${ }^{1}$ \\ ${ }^{1}$ Department of ECM, Koneru Lakshmaiah Education Foundation, \\ Green Fields, Vaddeswaram, A.P., India-522502.
}

\begin{abstract}
:
One of the main challenges in the Traffic Anomaly Detection (TAD) system is the ability to deal with unknown target scenes. As a result, the TAD system performs less in detecting anomalies. This paper introduces a novelty in the form of Adaptive Neuro-Fuzzy Inference System-LossyCount-based Topic Extraction (ANFIS-LCTE) for classification of anomalies in source and target traffic scenes. The process of transforming the input variables, learning the semantic rules in source scene and transferring the model to target scene achieves the transfer learning property. The proposed ANFIS-LCTE transfer learning model consists of four steps. (1) Low level visual items are extracted only for motion regions using optical flow technique. (2) Temporal transactions are created using aggregation of visual items for each set of frames. (3) An LCTE is applied for each set of temporal transaction to extract latent sequential topics. (4) ANFIS training is done with the back-propagation gradient descent method. The proposed ANFIS model framework is tested on standard dataset and performance is evaluated in terms of training performance and classification accuracies. Experimental results confirm that the proposed ANFIS-LCTE approach performs well in both source and target datasets.
\end{abstract}

Index Terms - Topic extraction, back propagation, shared data services 


\title{
An IoT based reference architecture for smart water management processes
}

\author{
Jasmitha Venna ${ }^{1}, \mathrm{vVdaya}$ sagar ketaraju ${ }^{2}$ \\ Department of CSE \\ Department of electronics and computer Engineering, \\ Koneu Lakshmaiah Educational Foundations(Deemed to be University) \\ e-mail:sagar.tadepalli@kluniversity.in
}

\begin{abstract}
Water is a vital resource for life, and its management is a key issue nowadays. Information and communications technology systems for water control are currently facing interoperability problems due to the lack of support of standardization in monitory and control equipment. This problem affects various processes in water management, such as water consumption, distribution, system identification and equipment maintenance. OPC UA (Object Linking and Embedding for Process Control Unified Architecture) is a platform independent service-oriented architecture for the control of processes in the logistic and manufacturing sectors. Based on this standard we propose a smart water management model combining Internet of Things technologies with business processes coordination and decision support systems. We provide an architecture for subsystem interaction and a detailed description of the physical scenario in which we will test our implementation, allowing specific vendor equipment to be manageable and interoperable in the specific context of water management processes.
\end{abstract}

Keywords: Water management, Irrigation, OPC UA, Internet of Things 


\title{
Emergency vehicles Pre-Emption
}

\author{
Madhav $\mathbf{M}^{1}$,Vdaya sagar ketaraju ${ }^{2}$ \\ Department of CSE, \\ Department of electronics and computer Engineering, \\ Koneu Lakshmaiah Educational Foundations(Deemed to be University) \\ e-mail:sagar.tadepalli@kluniversity.in
}

\begin{abstract}
Emergency Vehicle Pre-emption (EVP) systems play a key role in reprioritizing signalized traffic intersections. This role is important for safe and minimum travel delay of Emergency Vehicles (EV) passing through road intersections. Unfortunately, conventional systems that are currently in use on the roads have deficiencies in terms of cost, reliability, maintainability, and scalability. In this paper, a novel approach is proposed utilizing battery less Wireless Sensor Networks (WSNs) to overcome the above difficulties and improve the safety and mobility of emergency vehicles. A special purpose preemption protocol is designed based on the Collection Tree Protocol (CTP) as a data collection service. The protocol is called WSN-EVP and is used to handle the EV preemption request in a multihop tree-based topology spread across the traffic intersection. The model functions according to predefined rules and optimized parameters to cope with the high topology changes and the fast response time requirement. A prototype implementation using TelosB motes programmed in nesC shows a performance of high preemption accuracy under high level of interference.
\end{abstract}

Keywords: EVP,CTP, nesC, TelosB 


\title{
Smart Home Automation Security
}

\author{
Maleeha Khan,V Daya Sagar Ketaraju ${ }^{2}$ \\ Department of CSE, \\ Department of electronics and computer Engineering, \\ Koneu Lakshmaiah Educational Foundations(Deemed to be University) \\ e-mail:sagar.tadepalli@kluniversity.in
}

\begin{abstract}
This paper presents a comprehensive description about different home automation systems and technologies from a security standpoint. The work highlights various security flaws in existing home automation systems. In our work, we address how the concept of security and the meaning of the word "intruder" have evolved over time. We examine the challenges in home automation security from the point of view of both the homeowner and security engineer. The work goes on to explain why home automation systems are such attractive targets for an attacker. We point out the role of user interfaces in security. Various home automation technologies considered in our work include context-aware home automation systems, central controller-based home automation systems, Bluetooth-based home automation systems, Global System for Mobile communication or mobile-based home automation systems, Short Messaging Service-based home automation systems, General Packet Radio Service-based home automation systems, Dual Tone Multi Frequency-based home automation systems, and Internet-based home automation systems. The work concludes by explaining future directions home automation Security Research could take.
\end{abstract} Keywords: Smart Home, Access Control, Data Security, Intrusion Detection, User Interfaces 


\title{
Improving the verification of ontology authoring actions
}

\author{
Praveen $^{1}$, V Daya Sagar Ketaraju ${ }^{2}$ \\ Department of electronics and computer Engineering, \\ Koneu Lakshmaiah Educational Foundations(Deemed to be University) \\ e-mail:sagar.tadepalli@kluniversity.in
}

\begin{abstract}
Ontologies are complex systems of axioms in which unanticipated consequences of changes are both frequent, and difficult for ontology authors to apprehend. The effects of modelling actions range from unintended inferences to outright defects such as incoherency or even inconsistency. One of the central ontology authoring activities is verifying that a particular modelling step has had the intended consequences, often with the help of reasoners. For users of Protégé, this involves, for example, exploring the inferred class hierarchy. This paper provides evidence that making entailment set changes explicit to authors significantly improves the understanding of authoring actions regarding both correctness and speed. This is tested by means of the Inference Inspector, a Protégé plugin we created that provides authors with specific details about the effects of an authoring action. We empirically validate the effectiveness of the Inference Inspector in two studies. In a first, exploratory study we determine the feasibility of the Inference Inspector for supporting verification and isolating authoring actions. In a second, controlled study we formally evaluate the Inference Inspector and determine that making changes to key entailment sets explicit significantly improves author verification compared to the standard static hierarchy/frame based approach. We discuss the advantages of the Inference Inspector for different types of verification questions and find that our approach is best suited for verifying added restrictions where no new signature, such as class names, is introduced, with a $42 \%$ improvement in verification correctness.
\end{abstract}

Keywords: OWLOntologies,Human computer interaction,Ontology engineering,Ontology ,authoringReasoning 


\title{
Non-linear system identification using kernel based exponentially extended random vector functional link network
}

\author{
Tatiana Chakravorti ${ }^{1}$, P Satyanarayana ${ }^{2}$ \\ ${ }^{1,2}$ Department of ECM, Koneru Lakshmaiah Education Foundation, \\ Green Fields, Vaddeswaram, A.P., India-522502.
}

\begin{abstract}
:
Identification of nonlinear systems finds extensive applications in control design and stability analysis. To identify complex nonlinear systems, the neural network has drawn the attention of many researchers due to its broad application area. In this paper, an improved identification method based on Kernel Exponentially Extended Random Vector Functional Link Network (KERVFLN) has been proposed for nonlinear system identification. Good generalization capability, fast learling speed, simple architechture and the direct connection between input and output nodes along with non linear enhancement nodes with random weights of traditional Random Vector Functional Link Network (RVFLN) are very essential to industrial applications. To avoid the selection of the number of hidden nodes and hidden mapping function, kernal function has been used in this paper to increase the stability. The input is extended using trigonometric expansion which increases the accuracy of the algorithm when ever there is a sudden randon change. In case of KERVFLN the number of enhencement nodes and its corresponding activation function need not to be known if its corresponding kernel function is given. To verify the accuracy of the proposed model, some benchmark Monte Carlo simulations and one SISO system are carried out through simulation study and the obtained results are compared with some established techniques such as original RVFLN, Extreme Learning Machine (ELM), and Least Mean Square (LMS). The efficiency of the proposed technique has been tested with the real time data set as well.
\end{abstract}

Index Terms- Kernel Based Exponentially Extended RVFLN, system identification, water cycle algorithm, SISO system, Monte Carlo simulations 


\title{
Detection and Classification of COVID 19 using Transfer Learning with Convolutional Neural Network from Chest X-ray Images
}

\author{
Tatiana Chakravorti $^{1}$, Vinay Kumar Addala ${ }^{2}$ \\ 1,2 Department of ECM, Koneru Lakshmaiah Education Foundation, \\ Green Fields, Vaddeswaram, A.P., India-522502.
}

\begin{abstract}
:
In 2019 the novel Corona virus which is called COVID 19 was originated in China and now it has spread all over the world. So far more than $27 \mathrm{M}$ people got affected all over the world and as the number of cases are increasing exponentially, most of the countries are facing shortage of test resources. In India the number of confirmed infected cases by COVID 19 is $4.28 \mathrm{M}$ where more than 72,700 people died till $9^{\text {th }}$ Sep 2020 . The increasing number of daily COVID 19 cases needs another solution and needs a lot of study. In this paper a deep learning model has been proposed for detection and classification of COVID 19 with high accuracy from chest x-ray. The tensor flow based CNN method has been proposed to classify using chest x-ray images. The proposed model has been trained and tested on the prepared dataset and it has been found that the overall accuracy achieved for the 2-class classification (COVID 19 vs Healthy) is 95\%, and more importantly the precision and recall rate for COVID-19 cases both are $95 \%$. To prove the robustness of the model, it has been tested for 3-class (COVID vs Pneumonia vs Healthy) as well as the 4-class (COVID vs Healthy vs Pneumonia virus vs Pneumonia Bacterial) classification. The achieved overall accuracy for 3 -class is $96 \%$ and the 4-class is $91.3 \%$ where the recall and precision is more than $95.7 \%$. The performance of the proposed model is very promising and it can be very helpful tool for radiologists and clinical practitioners in case of COVID 19 detection and classification because of its simplicity. More over this paper represent a comparative study of the proposed technique with ELM which will help researchers for further analysis.
\end{abstract}

Keywords: Chest x-ray images, Tensor flow, Convolutional Neural Network, Detection, Classification 


\title{
Classification of Disturbances in a PV based Distributed Generation System using Cross VMD and Random Vector Functional Link Network
}

\author{
Tatiana Chakravorti ${ }^{1}$ \\ ${ }^{1}$ Department of ECM, Koneru Lakshmaiah Education Foundation, \\ Green Fields, Vaddeswaram, A.P., India-522502.
}

\begin{abstract}
:
This paper presents power quality (PQ) disturbance detection and classification along with islanding for a grid connected distributed generation system where the threshold has been determined depending upon the PV penetration. To design effective pattern recognition the microgrid is modelled according to IEEE 1547 and different events has been simulated. To detect and classify the disturbances a Cross Variational Mode Decomposition (XVMD) with Random Vector Functional Link Network (RVFLN) has been proposed in this paper as a new contribution to the literature. The features have been extracted from the output of the XVMD and the significant distinguishable features have been given to the classifier input. Ten different types of disturbances have been generated to test the robustness of the proposed hybrid technique with different noisy conditions as well. It has been found that the proposed method is very much acceptable for the classification of disturbances with high accuracy.
\end{abstract}

Index Terms - Cross Variational Mode Decomposition (XVMD), Disturbance classification, Random Vector Functional Link Network (RVFLN) 


\title{
Real-time Anomaly Detection using Tensorflow based RNN Deep Learning Classifier
}

\author{
Balla Mani Chidvilas ${ }^{1}$, Kancharla Sai Pavan ${ }^{2}$, Sanaka Naga Sai Kiran ${ }^{3}$, MerugaMourya Kanth ${ }^{4}$, V S \\ Bhagavan $^{5},{ }^{*}$ Tatiana Chakravorti ${ }^{6}$ \\ 1,2,3,4,6 Electronics and Computer Science Engineering,KoneruLakshmaiah Education Foundation, \\ Vijayawada, Andhra Pradesh 522502, India, \\ ${ }^{5}$ Department of Mathematics, KoneruLakshmaiah Education Foundation, Vijayawada, Andhra Pradesh \\ 522502, India
}

\begin{abstract}
:
Abstract-In this paper, a TensorFlow based Deep Learning classifier has been introduced for realtime anomaly detection to improve the commercial and national security. It becomes even more complicated and sophisticated when dealing with highly populated countries like India. Our proposed prototype consists of 4 modules namely data organization, video to frame conversion, feature extraction and model evaluation. UCF Crime Dataset is chosen as it contains a variety of video clips based on real-world anomalies to make sure the outcomes are tangible. The entire dataset of videos is transformed into individual frames using the FFmpeg library to get the features from each frame. Using Convolutional Neural Networks, the feature extraction process is made possible and the data is saved for training. The features are appended to the frames in a fixed sequence which is fed to LSTM to train the entire dataset. Added callbacks ensure that the model saves the best weights in each epoch to attain more accuracy. It has been found that the proposed model is very much acceptable for anomaly detection and gives good accuracy than some already established algorithms.
\end{abstract}

Keywords-TensorFlow, Deep Learning, Anomaly Detection, Classification 


\title{
Smart Home Automation using IOT
}

\author{
J. Rajasekhar ${ }^{1}$, N.Sowjanya ${ }^{2}$ \\ Assistant professor1* Department of Electronics and communication engineering, KLEF (Deemed to be University), Vaddeswram, Guntur \\ District, Andhra Pradesh \\ Scholar in 2* Department of Electronics and communication engineering, KLEF (Deemed to be University), Vaddeswram, Guntur District, \\ Andhra Pradesh \\ Email: rajasekharemb@gmail.com, srisowjanya16@gmail.com
}

\begin{abstract}
:
In recent times the life of humans beings have become very simple because of the automation is havening in the every domain of the human life. In this paper we are using the IOT to bring the "intelligence" to the devices in home to make a smart home. The smart water take care of the home water tank in home, the gardening control system, appliance control system and automatic light control system based on number of people in the room, measuring the co levels in the room and outdoor, garbage management system in the home, fish health monitoring system in the fish tank, this paper identifies the features of the smart home and by providing the flexibility to the IOT devices to communicate among themselves and operate the home appliances
\end{abstract}

Keywords: IOT, sensors, actuators. Smart home 


\title{
An approach to hybridisation of Embedded System Networks
}

\author{
J. Rajasekhar, Dr. JKR Sastry, and N. Sowjanya \\ Department of Electronics and Computer engineering, KLEF (Deemed to be University), \\ Vaddeswram, Guntur District, Andhra Pradesh
}

\begin{abstract}
Embedded systems can be networked using either wired or wireless technologies. ES systems when networked using wires can communicate serially over a bus using the technologies such as CAN, I ${ }^{2} \mathrm{C}, \mathrm{USB}, \mathrm{RS} 485$, and Fire wire. These standards differ in many ways which include arbitration, synchronisation, address resolution, timing, type of communication etc. Embedded systems can also be network using wireless technologies which exits in many versions. Several applications these days are requiring more than one communication technology. Several subsystems are developed using a networking method and it requires that the subsystems that are networked using different technologies are to be networked further for realising entire application. Such a network needs to deal with many of the heterogeneous communication system leading to an issue of hybridisation. In this paper various issues/approaches that need to be addressed for hybridising of the ES networks have been presented.
\end{abstract}

Key words: ES networking, hybridization, interconnection of heterogeneous networks 


\title{
Access Control of Door using Face Recognition And Home Security Alert Using Raspberry Pi And Internet
}

\section{Gorantla Sai Gopi, Palla Priyanka, K. Bharathi, J.Rajasekhar, Krishnaveni Kommuri}

\author{
Department of Electronics and Computer Engineering, Koneru Lakshmaiah Education \\ Foundation, Guntur, India.
}

\begin{abstract}
:
In the present age, the Internet of things (IOT) has entered a brilliant period of quick development. The Internet of things is an idea that expects to expand the advantages of the ordinary Internet steady network, remote control capacity, information sharing, etc. to products in the physical world. Ordinary things are getting associated with the Internet. This idea can be utilized to manage the security concerned issues in a financially way. In this paperwork a framework is being created to interface any entryway with the Internet, so the entrance control framework can be controlled from anyplace on the planet. For a situation that one isn't at home and a visitor is at his entryway steps then the approved individual will be told about the guest by means of IFTTT app, which pings up a message to your from anyplace and the framework IFTTT snap a photo of the guest and keep a record by sending a connection through Email. On the off chance that the approved individual needs to give a message the guest, it tends to be sent effectively through the IFTTT app and it will show up in a screen on the front essence of the entryway. The entryway lock can be controlled through the IFTTT. With the assistance of this framework, a proof of the guest can be kept as a record if any crisis case or situation happens.
\end{abstract}



Engineering and Management Research

A Peer Revieved Open Access International Journal

\title{
Recognition of Vehicle Number Plate and Measure the Distance
}

\author{
B. Jyothi Sravya, V. Naga Lakshmi, J. Rajasekhar \\ Department of Electronics and Computer Engineering, Koneru Lakshmaiah Education \\ Foundation, Vaddeswaram, Guntur Dist, A.P India.
}

\begin{abstract}
:
Now a days most of the developing cities most of the accidents are happening frequently because of not following traffic rules so in this paper we have found a solution to avoid this kind of problems if any vehicle jumps the red signal is on then automatically start the camera. And capture the image of the vehicle and extract the number plate from the vehicle and send it to the data base automatically and send the payment amount and link as SMS to the vehicle owner which includes date and time. To use the ultrasonic sensor which will be helpful for calculating the distance between zebra crossing line and vehicles.
\end{abstract}

Keywords: Raspberry pi, Ultrasonic sensor, Traffic density, Open CV, OCR (optical character recognition). 


\title{
Modeling of GPS-TEC using QR-decomposition over the low latitude sector during disturbed geomagnetic conditions
}

\section{J.R.K. Kumar Dabbakuti ${ }^{1}$, Y. Mallika ${ }^{2}$, M. Venugopala Rao ${ }^{2}$, K. Raghava Rao $^{1}$, D. Venkata Ratnam ${ }^{2}$}

\author{
${ }^{1}$ Department of ECM, Koneru Lakshmaiah Education Foundation, Green Fields, Vaddeswaram, \\ A.P., India-522502. \\ ${ }^{2}$ Department of ECE, Koneru Lakshmaiah Education Foundation, Green Fields, Vaddeswaram, \\ A.P., India-522502.
}

\begin{abstract}
:
Given the continuous operation of satellite-based navigation applications, modeling of Total Electron Content (TEC) during magnetic disturbed periods is a significant challenge. The Global Positioning System (GPS)- TEC observations of the Bangalore station $\left(13.02^{\circ} \mathrm{N}, 77.57^{\circ} \mathrm{E}\right)$ was considered and covers the period (2009 to 2016) of the solar cycle 24 . The study emphases on the analysis of TEC variations in eight geomagnetic storms of different intensity: $(-223 \mathrm{nT}<\mathrm{Dst}<-80$ nT). In this Paper, QR decomposition is computed using the Gram-Schmidt (GS) process on equatorial/low latitude sectors during disturbed geomagnetic conditions. For interpolation, the QR model was evaluated on storms that occurred during different periods of solar activity (2009 to 2016), while for extrapolation the assessment was conducted for the most intensive storm March 17, 2015 (St. Patrick's Day storm: Dst $-223 \mathrm{nT}$ ) at different latitudes, covering $10^{\circ} \mathrm{N}$ to $26^{\circ} \mathrm{N}$ on the Asian longitude. The R1 and Q1 modes patterns are consistent with changes in the solar proxy index (F10.7) and with regular daily variations and the correlation coefficient is 0.80 and 0.99 . The post-residue between the QR model TEC and the GPS- TEC values is \pm 3 TECU. The QR model well captured the TEC responses in consecutive storms case (18 to 24, February 2014). The spatial variation of the TEC deviations increases as it moves towards the crest Equatorial Ionization Anomaly (EIA) from the magnetic equator and decreases beyond the crest. The proposed work is valuable for the further study of the Global Navigation Satellite System (GNSS) performances during geomagnetic magnetic disturbed periods.
\end{abstract}

Index Terms - Modeling, QR model, GNSS, Disturbed geomagnetic conditions. 


\title{
Application of Singular Spectrum Analysis Using Artificial Neural Networks in TEC Predictions for Ionospheric Space Weather
}

\author{
J.R.K. Kumar Dabbakuti ${ }^{1}$, and Bhavya Lahari $\mathbf{G}^{1}$ \\ ${ }^{1}$ Department of ECM, Koneru Lakshmaiah Education Foundation, Green Fields, Vaddeswaram, \\ A.P., India-522502.
}

\begin{abstract}
:
The prediction of the ionospheric state has become increasingly important as more and more terrestrial, and space-based radiocommunication systems rely on ionospheric space weather. This paper presents a new ionospheric prediction model, named the Singular Spectrum Analysis Artificial Neural Network (SSA-ANN) model. SSA is used as a pre-processing tool for the ionosphere Total Electron Content (TEC) prediction based on the ANN approach. The hourly Global Positioning System (GPS) -TEC observations from 2009 to 2017 period at Bangalore $\left(13.02^{\circ} \mathrm{N}\right.$ and $\left.77.57^{\circ} \mathrm{E}\right)$ station are taken into account for the analysis. The quick convergence of the SSA decomposition makes it possible to use the first four SSA modes to represent $99.57 \%$ of the total variance of the GPS-TEC data set. The Root Mean Square Error (RMSE) between observed and SSA-ANN model TEC value is 1.40 TECU for the period (2009-2017), and the correlation coefficient is 0.99 . The performance of SSA-ANN is evaluated using Autoregressive Moving Average (ARMA) and International Reference Ionosphere (IRI-16) models in three different cases of solar phase periods, namely the Ascending Solar Phase (ASP), High Solar Phase (HSP) and Descending Solar Phase (DSP) and in different seasons. The SSA-ANN model predicts the absence of winter anomaly during the ASP period (2012), but the ARMA model and IRI models failed to predict the absence of anomaly. The proposed SSA-NN model resulted in higher prediction accuracy and reduced training and testing times, which would be useful for the development of an ionospheric predicting system.
\end{abstract}

Index Terms - Singular Spectrum Analysis, Artificial Neural Network, Autoregressive Moving Average, Prediction model 


\title{
Implementation of IoT Analytics Ionospheric Forecasting System based on Machine Learning and ThingSpeak
}

\author{
J.R.K. Kumar Dabbakuti ${ }^{1}$, Abin Jacob ${ }^{1}$, V. Venkata Rao ${ }^{2}$, K Ravi Kumar ${ }^{1}$ \\ ${ }^{1}$ Department of ECM, Koneru Lakshmaiah Education Foundation, Green Fields, Vaddeswaram, \\ A.P., India-522502. \\ ${ }^{2}$ Department of ECE, NEC, Guntur, Andhra Pradesh, India.
}

\begin{abstract}
:
Nowadays using the Internet of Things (IoT), several real-time forecasting systems have been developed. The primary challenge of this system is to utilize an appropriate prediction model which can predict various space weather parameters as accurately as possible. In this paper, an ionospheric IoT analytical system with Variational Mode Decomposition (VMD) based on Kernel Extreme Learning Machine (KELM) is proposed. The ionospheric signal delay/ Total Electron Content (TEC) data from Continuous Reference Stations (CORS) Port Blair $\left(2.03^{\circ} \mathrm{N}, 165.25^{\circ} \mathrm{E}\right.$, geomagnetic), Bengaluru (4.40 $\mathrm{N}, 150.77^{\circ} \mathrm{E}$, geomagnetic), Koneru Lakshmaiah Education Foundation (KLEF) - Guntur $\left(7.50^{\circ} \mathrm{N}, 153.76^{\circ} \mathrm{E}\right.$; geomagnetic) and Lucknow $\left(17.98^{\circ} \mathrm{N}, 155.22^{\circ}\right.$ E; geomagnetic) are used for the analysis during the period of 2015. The ionospheric signal delays of four CORS are computed from ThingSpeak (IoT) with the channel ID and the Application Programming Interface (API) key. ThingSpeak data is given to the ionospheric forecasting model (VMD-KELM). The results predicted from the proposed model are able to achieve the faster training process and obtain a similar accuracy to that of the VMD- Artificial Neural Network (ANN). The proposed VMD-KELM application is adopted when a cloud-based forecasting system requires fast learning speed and good accuracy. As a result, the cloud paradigm offers the possibility without web development skills or highly specific statistics.
\end{abstract}

Index Terms - Kernel Extreme Learning Machine, Artificial Neural Network, Variational Mode Decomposition, Total Electron Content 


\section{Ionospheric Monitoring System based on the Internet of Things with ThingSpeak J.R.K. Kumar Dabbakuti ${ }^{1}$, Bhupathi $\mathrm{Ch}^{1}$}

${ }^{1}$ Department of ECM, Koneru Lakshmaiah Education Foundation, Green Fields, Vaddeswaram, A.P., India-522502.

\section{ABSTRACT:}

The Internet of Things (IoT) is a growing technology that allows digital devices to be integrated into the network. The use of this IOT technology makes it possible to gather information from various data of the Global Navigation Satellite System (GNSS) receiver connected to the Internet, which constitutes a unique opportunity to obtain information on the spatial and temporal distribution. In this Paper, Cloud-based (IOT) ionospheric monitoring system using ThingSpeak have proposed. The ionospheric signal delay/Total Electron Content (TEC) data from GNSS stations, Koneru Lakshmaiah Education Foundation (KLEF)-Guntur (7.50 ${ }^{\circ} \mathrm{N}, 153.76{ }^{\circ} \mathrm{E}$; geomagnetic), Port Blair (2.03 ${ }^{\circ} \mathrm{N}, 165.25^{\circ} \mathrm{E}$; geomagnetic), Bengaluru $\left(4.40{ }^{\circ} \mathrm{N}, 150.77^{\circ} \mathrm{E}\right.$; geomagnetic) Lucknow (17.98 ${ }^{\circ} \mathrm{N}, 155.22^{\circ} \mathrm{E}$; geomagnetic) are used for the analysis during the solar cycle 24 during of 2015 period. The ionospheric signal delay is computed from the ThinkSpeak (IOT), and the ionospheric TEC calculations are performed directly in the MATLAB software. Therefore, the Cloud paradigm offers the opportunity to develop advanced applications where their accurateness makes them appropriate for an implementation based on cloud-based infrastructure. 


\title{
Water leakage detection monitoring and controlling system using IOT
}

\author{
B Swetha Reddy, P.Email Author, Chanakya, K.V., Eswari, B., Bhupati, \\ Department of ECM, Koneru Lakshmaiah Education Foundation, \\ Green Fields, Vaddeswaram, A.P., India-522502.
}

\begin{abstract}
:
The Water is the most valuable because it is a basic need of all humans, in these days water supply department are facing problem because of less amount of water due to less rainfall, lowering of ground water in spite of all these problems water leakage from pipelines while supplying and also no monitoring of water distribution and controlling. With increment in population, neighborhoods have expanded as a result of this reason water has turned into a major issue which influences in distribution, conservation, consumption and also the quality so, to overcome all these problems and create system economical there's want of correct observance and dominant system. In this paper, we are proposing a system which can detect the leakages, monitoring and controlling if water supply in pipelines. Sensors places in the tank which continuously informs the water level of water in the tank. The motor functioning will be automatically turn on and when tank is about to fill up it will cut off.
\end{abstract}

Index Terms - Arduino uno, ESP8266, Flow Sensor, GSM, LCD 


\title{
Broadcast- based Data Gathering Mechanism for Sampling Sensor Fields
}

\author{
Ali Hussain, M. , Anusha, M. Vamsi Krishna. \\ Department of Electronics and Computer Engineering, Koneru Lakshmiah Educational \\ Foundation, Guntur, Andhra Pradesh, India
}

\begin{abstract}
:
Wireless sensor networks are a new technology that can provide processed real-time field data from sensors physically distributed in the field. The applications of wireless sensor networks are Military or Border Surveillance, Health Care, Environmental Conditions Monitoring, Home Intelligence, Industrial Process Control, Agriculture, etc., In a sensor field, the sensor nodes act as sources of the sensor data. To gather samples of data from a sensor field, a mobile object can be used. This mobile object is called a mobile sink. A critical task is how to group the sensor data from the sensor nodes. An algorithm called Band- based directional broadcast is used to manage the broadcast paths from the sensor nodes. To reduce the chance of packet loss and make the task energy efficient, an efficient routing protocol called Dynamic Source Routing protocol is used. This paper presents an overview of the Band- based directional broadcast and how it can be used energy efficient.
\end{abstract}

Index Terms -DSR, Mobile node, Sensors, WSN 


\title{
A Study of Intrusion Detection Systems in Heterogeneous Wireless Sensor Networks
}

\author{
Ali Hussain, M. Byasani Naga Kalki Venkatesh. \\ Department of Electronics and Communication Engineering, Koneru Lakshmiah Educational \\ Foundation, Guntur, Andhra Pradesh, India
}

\begin{abstract}
:
Wireless sensor networks (WSNs) composed of smart sensors interconnected over wireless links are quickly becoming the technology of choice for monitoring and measuring geographically distributed physical, chemical, or biological phenomena in real time. These are deployed for monitoring in a range of critical domains for example health care, military, critical infrastructure. Wireless Sensor Networks are homogeneous or heterogeneous systems. A Heterogeneous WSN is more complex as compared to homogeneous WSN and which consists of a number of sensor nodes of different types deployed in a particular area and which are collectively working together to achieve a particular aim. The aim may be any of the physical or environmental condition. For e.g. the wireless sensor network is mainly used in military applications such as in borders for finding out the infiltrations. It is also used in industrial process monitoring and control, machine health monitoring, environment and habitat monitoring, healthcare applications, home automation and traffic control. Wireless sensor networks are vulnerable to many attacks. This paper presents an overview of the intrusion detection in heterogeneous wireless sensor networks.
\end{abstract}

Index Terms-Heterogeneous WSN, Intruders, IDS, Sensors. 


\title{
Detection of Active Internet Worm: Camouflaging Worm
}

\author{
K V D Sagar, CHERUKURI LEKHYA. \\ Department of Electronics and Computer Engineering, Koneru Lakshmiah Educational \\ Foundation, Guntur, Andhra Pradesh, India.
}

\begin{abstract}
:
Internet worms are truly autonomous virtual viruses, spreading across the net, breaking into computers, and replicating without human assistance and usually without human knowledge. Worms are particularly interesting technological constructs, with an intriguing mathematical structure and complexity. They fascinate because they take the digital imitation of life to another step - they autonomously search for computers, penetrate them, and replicate their intelligence to continue the process. An active worm refers to a malicious software program that propagates itself on the Internet to infect other computers. The propagation of the worm is based on exploiting vulnerabilities of computers on the Internet. The camouflaging worm, also called CWorm, is a type of active internet worm. A C- Worm can intelligently manipulate its scan traffic volume over time. Thus a $\mathrm{C}$ - Worm can camouflage its propagation form existing worm detection systems based on analyzing the propagation traffic generated by worms. This paper presents a method to detect C- Worms.
\end{abstract}

Index Terms -Camouflaging, Detection, Propagation, Worm. 


\title{
A Study of Intrusion Detection Systems in Heterogeneous Wireless Sensor Networks
}

\author{
Naga Venkata Srinivas Kale, M. Vamsi Krishna, K.V.Ramana \\ Department of Electronics and Computer Engineering, Koneru Lakshmiah Educational \\ Foundation, Guntur, Andhra Pradesh, India
}

\begin{abstract}
:
Wireless sensor networks (WSNs) composed of smart sensors interconnected over wireless links are quickly becoming the technology of choice for monitoring and measuring geographically distributed physical, chemical, or biological phenomena in real time. These are deployed for monitoring in a range of critical domains for example health care, military, critical infrastructure. Wireless Sensor Networks are homogeneous or heterogeneous systems. The existing sensor fields communication will not be reduce a traffic issues to compare use of the single and multiple sensor fields. The heterogeneous is shrinking of the network nodes will not be supported to detect the intrusions. A Heterogeneous WSN is more complex as compared to homogeneous WSN and which consists of a number of sensor nodes of different types deployed in a particular area and which are collectively working together to achieve a particular aim. The aim may be any of the physical or environmental condition. For e.g. the wireless sensor network is mainly used in military applications such as in borders for finding out the infiltrations. It is also used in industrial process monitoring and control, machine health monitoring, environment and habitat monitoring, healthcare applications, home automation and traffic control. Wireless sensor networks are vulnerable to many attacks. This paper presents an overview of the intrusion detection in heterogeneous wireless sensor networks.
\end{abstract}

Index Terms -Heterogeneous WSN, Intruders, IDS, Sensors. 


\title{
Delay Analysis
}

\author{
Ali Hussain M., Badam Sampath \\ Department of Electronics and Computer Engineering, Koneru Lakshmiah Educational \\ Foundation, Guntur, Andhra Pradesh, India
}

\begin{abstract}
:
A Multi- hop wireless network is a wireless network adopting multihop wireless technology without deployment of wired backhaul links. In multi- hop wireless networks, the routes between source and destination pairs are relatively fixed. In such networks, a metric called delay is of prime importance. But the delay analysis of multi- hop wireless networks has largely been an open problem. This problem is notoriously difficult even in the context of wireline networks, primarily because of the complex interactions in the network (e.g., superposition, routing, departure, etc.) that make its analysis amenable only in very special cases like the product form networks. Maximizing the network throughput, or equivalently, attaining the maximum stability region of the network, through appropriately scheduling is a key design goal in wireless networks. But the problem of delay analysis further exacerbated by the mutual interference inherent in wireless networks which, complicates both the scheduling mechanisms and their analysis. This paper focuses on the delay analysis of a queue length based scheduling policy in multi- hop wireless network.
\end{abstract}

Index Terms -Heterogeneous WSN, Intruders, IDS, Sensors. 


\title{
Family based efficient routing protocol (FERP) for lifetime improvement in heterogeneous wireless sensor networks
}

\author{
Srikanth, N., Prasad, M.S.G. \\ ${ }^{1}$ Department of Electronics and Computer Engineering, Koneru Lakshmiah Educational \\ Foundation, Guntur, Andhra Pradesh, India
}

\begin{abstract}
:
WSN has opened an attracting possibility of transforming gross mechanical actions into subtle sensory responses. WSN consists of group of nodes which does not have manual maintenance, and their energy resources follow scavenging Principle. Energy efficiency is an important parameter in WSN which leads to improve network lifetime, link quality, and throughput. Efficient energy utilization by clustering nodes is greatly influenced by election of cluster head. Even there is a high level of research from few decades; still WSN faces so many issues like high energy consumption, lack of lifetime improvement, low QOS, less throughput, etc. There are several clustering, routing, cluster based routing techniques to make the network more energy efficient. This paper explores different cluster based routing in military areas and plateaus. This cluster based routing gives effective results compared with traditional protocols and gives lifetime 62\%, and energy consumption 47\%. () 2019, Institute of Advanced Scientific Research, Inc.. All rights reserved.
\end{abstract}

Index Terms - Cluster-based RoutingFERPLifetimeThroughputWSN 


\title{
Green CoMP based energy efficient data aggregation algorithm with malicious node identification (geed- m) for lifetime improvement in WSN
}

\author{
Srikanth, N., Prasad, M.S.G. \\ Department of Electronics and Computer Engineering, Koneru Lakshmiah Educational \\ Foundation, Green fields, Vaddeswaram, India
}

\begin{abstract}
:
Random deployment of sensor nodes, energy limitations, interference of wireless links, and exposed nodes, are the major reasons of performance degradation in WSN. Energy efficiency, Lifetime improvements are the key research areas from last few decades. Even a high level of research is going on; still there are several issues which reduce the network lifetime and its throughput. For diminishing the energy consumption, the sensor nodes are driven into sleep mode once they finished their sensing round. Introducing mobile nodes in sub clusters is an efficient technique to make the network energy efficient in irregular terrains like plateaus. The energy limitations of mobile nodes, and malicious behaviour are big issues in mobile node based sub clustered sensor networks. These issues can be clear up by introducing a Green CoMP based energy efficient data aggregation algorithm with malicious node identification is proposed, which exchange messages to the cluster head through a mobile node. Malicious behaviour of mobile node is also identified by using Built-in self-Test based technique to improve network throughput. The proposed algorithm gives better results compared with existing algorithms with a lifetime improvement of 56\%, energy consumption 44\% . () 2019 COMPUSOFT, An international journal of advanced computer technology.
\end{abstract}

Index Terms - Built-in self-test, Data aggregation, Green-CoMP, Lifetime, Malicious node 


\title{
Analysis of throughput and spectrum handoff delay in cognitive radio
}

\author{
${ }^{1}$ Katta, S., ${ }^{2}$ Siva Ganga Prasad, M., ${ }^{1}$ Madhav, B.T.P. \\ ${ }^{1}$ Department of ECE, Koneru Lakshmaiah Education Foundation, Vaddeswaram, Andhra \\ Pradesh, India \\ ${ }^{2}$ Department of ECE, KKR \& KSR Institute of Technology \& Sciences, Guntur, Andhra \\ Pradesh, India
}

\begin{abstract}
:
The problem of spectrum scarcity can be solved by Cognitive Radio. In Cognitive Radio, the spectrum handoff technique is used to reduce the interference to Primary User by Secondary User and to utilize the unused spectrum dynamically. The traditional handoff scheme consists of two phases, sensing and transmission. A spectrum sensing-throughput trade off scheme is considered and the two optimization problems are proposed to increase the throughput and decrease the spectrum handoff delay. Optimum values of sensing time, searching time and number of channels are obtained with TLBO algorithm. Analyze the effect of probability of idle channel $\mathrm{P}(\mathrm{H} \mathrm{o})$ and number of channels on handoff delay. Compare the results of proposed optimization method TLBO with traditional and sensing-throughput trade off scheme. @ 2018, Institute of Advanced Scientific Research, Inc. All rights reserved.
\end{abstract}

Index Terms - Cognitive radio, Spectrum handoff, Throughput, TLBO 


\title{
Implementation of distributed power control/active link protection for femto cell networks
}

\section{Cheerla, S., Venkata Ratnam, D., Siva Ganga Prasad, M., Vyshanvi, B.,Sai Nitin, A.}

Department of ECE, KLEF, KL University, Vaddeswaram, Guntur, Andhra Pradesh, 522502, India

\begin{abstract}
:
Femto cell plays major role in the modern wireless communication systems applications. Femto cell provides higher data rates with a tiny base station. The prime concern in femto cell is power allocations for mobile receiver due to interferences. There is a need to develop a suitable power allocation algorithm to improve performance of femto cells. In this paper, an optimized power algorithm Distributive Power Control with Active Link Protection (DPC/ALP) is implemented. The proposed algorithm is tested under MATLAB simulated Long Term Evolution (LTE) environment and also evaluated with existing power allocation techniques i.e. fixed power scheme and power adaptive SINR. It is found that $39 \%$ improvement of power optimization is obtained when compared to the other techniques. The outcome of this research work would be immensely useful for developing robust power optimization techniques for femto cells.
\end{abstract}

Index Terms - Active link protection (ALP), Distributed power control, Femto cells, LTESINR 


\title{
Analysis of different Direction of Arrival (DOA) estimation techniques using smart antenna in wireless communications
}

\author{
Prasad, M.S.G., Siddaiah, P., Reddy, L.P. \\ Dept. ECE, Koneru Lakshmiah Education Foundation, Guntur, Andhra Pradesh, India
}

\begin{abstract}
:
Smart antenna systems plays an important role in wireless communications systems. This paper begins with a brief introduction about an Array correlation matrix in the wireless communication. After that, the goal of Direction Of Arrival (DOA) estimation with smart antennas in the wireless communications systems is described. The three different types of DOA estimation techniques Bartlett DOA estimation, Minimum Variance Distortionless Response (MVDR) and Linear prediction DOA estimation techniques are described in the fourth part of this paper. In the last section of this paper comparison of their performance evaluation of these three methods and conclusion are made. A femto cell plays major role in the modern wireless communication systems applications. Femto cell provides higher data rates with a tiny base station. The prime concern in femto cell is power allocations for mobile receiver due to interferences.
\end{abstract}

Index Terms - DOA, Estimation, Smart antenna, Wireless communications 


\title{
Clock synchronization in an $\mathrm{N}$-modular redundant system
}

\author{
K. Vijayalaxmi ${ }^{1}$, K. V. Ratnam ${ }^{2}$ \\ ${ }^{1}$ Raghu Engineering College, Visakhapatnam, AP,India. \\ ${ }^{2}$ Department of ECE, Koneru Lakshmaiah Education Foundation, \\ Green Fields, Vaddeswaram, A.P., India-522502.
}

\begin{abstract}
:
High integrity computer systems such as those found in aircraft and spacecraft often rely on fault tolerance to maintain functionality in the presence of one or more faults. A common strategy consists in comparing the output of functionally identical computers, allowing the output of healthy computers to mask out the output of faulty computers. This approach is generally known as N-Modular redundancy and requires that the redundant computers maintain a high degree of synchronization, as comparing the output of well-functioning but out sync computers would defeat the fault detection strategy. The challenge is to devise a robust method for keeping healthy computers synchronized in the presence of potentially malfunctioning computers. The novice might suggest synchronizing the redundant computers to a common hardware clock, but this leads to a single point of failure should this hardware clock become faulty. The solution is not to rely on one hardware clock, but to synchronize the clocks in the redundant computers, thereby defining a distributed global common clock. This paper describes the clock synchronization strategy and implementation used in the Redundancy Management System (RMS) developed at Honeywell Aerospace Electronic Systems.
\end{abstract}

Index Terms - Computer, N-modular, RMS 


\title{
Development of Internet of Things based Decision Support for Vehicle Drivers by using GPS and GSM
}

\author{
A. Kalyani ${ }^{1}$, K. V. Ratnam ${ }^{2}$ \\ ${ }^{1}$ Department of Electronics \& Communication Engg, K L E F, Vaddeswaram, AP, India, \\ 2 Department of Electronics \& Computer Science Engg, K L E F, Vaddeswaram, AP, India
}

\begin{abstract}
:
This article explains about development of Internet of Things (IoT) based decision support for vehicle drivers using GPS and GSM modules. This project is helpful to avoid the road accidents by maintaining the proper speed limit at different locations such as school zones, hospital regions and so on. Initially an admin database is created with a web server. The data base contains six parts such as S.No, longitude1, latitude1, longitude2, latitude2, speed limit. The web server has been implemented with a PHP page which provides a connection to the databases allowing web clients to send queries to data base. A PC application is distributed among local guides; they can provide speed limits of the allocated regions. A GPS receiver is used to provide the vehicle's location and a GSM module is configured as GPRS to provide internet connection through mobile data. An Organic Light Emitting Diode (OLED) is used to display the speed limit of the vehicle's location. Arduino UNO (At mega 328P) board is used to interface all the components. The instructions to the vehicle drivers are given by using OLED display when the location is tracked by GPRS, and also an alarm sounds at extreme conditions.
\end{abstract}

Index Terms - Adaptation, Cloud, GPS, GSM, IoT, OLED 


\title{
Intrusion Detection System using AI and Machine Learning
}

\section{Algorithm}

\section{R. Sai Akhil ${ }^{1}$, K. V. Ratnam ${ }^{2}$}

${ }^{1}$ Department of Electronics \& Communication Engg, K L E F, Vaddeswaram, AP, India, 2 Department of Electronics \& Computer Science Engg, K L E F, Vaddeswaram, AP, India

\begin{abstract}
:
Secure automated threat detection and prevention is the more effective procedure to reduce the workload of analyst by scanning the network, server functions \& then informs the analyst if any suspicious activity is detected in the network traffic. It monitors the system continuously and responds according to the threat environment. This response action varies from phase to phase. Here suspicious activities are detected by the help of an artificial intelligence which acts as a virtual analyst concurrently with network intrusion detection system to defend from the threat environment and taking appropriate measures with the permission of the analyst. In its final phase where packet analysis is carried out to surf for attack vectors and then categorize supervised and unsupervised data. Where the unsupervised data will be decoded or converted to supervised data with help of analyst feedback and then auto-update the algorithm (Virtual Analyst Algorithm). So that it evolves the algorithm (with Active Learning Mechanism) itself by time and become more efficient, strong. So, it can able to defend form similar or same kind of attacks.
\end{abstract}

Index Terms - AI, IDS, ML 


\title{
Implementation of IoT based vehicle theft detection and accident monitoring system using Arduino
}

\author{
Krishna Manogna ${ }^{1}$, K. V. Ratnam ${ }^{1}$ \\ 1 Department of Electronics \& Computer Science Engg, K L E F, Vaddeswaram, AP, India
}

\begin{abstract}
:
This paper presents an efficient system which detects the vehicle accident and sends intimation to the registered mobile numbers such as ambulance or vehicle owner or police etc. IOT based vehicle tracking system detect the Accidents and it sends the position of the vehicle to the specified or pre-programmed mobile number as an SMS. Not only prohibited the accidents. It also prevents the vehicle from theft. If we want opening the door of the vehicle then we need to press a button. Now the owner of the vehicle gets an SMS to grant access. The door will be opened after the authentication only. If an unknown one tries to open the door of the vehicle then you can restrict that person by using mobile. The GPS is used for tracking the vehicle in this system. If any accident detected then an alert will be given with buzzer. Now the driver can press button to stop that. If it is small accident, here the driver can stop the buzzer if the vehicle is safe with in time period. Otherwise an alert will be sent to the owner of the car. It indicates that the person in car or car in trouble.
\end{abstract}

Index Terms - GPS, Mobile, SMS. 


\title{
Tokenization of news feed articles based on their similarity using machine learning techniques
}

\author{
Deepika, V., Kameswara Rao, M., Kiranmai, N. \\ Department of ECM, Koneru Lakshmaiah Education Foundation, \\ Green Fields, Vaddeswaram, A.P., India-522502.
}

\begin{abstract}
:
Now a days, we find huge amount of similar data in world wide web. To resolve this we use document similarity measures like information retrieval, plagiarism detection, document clustering. So, to check this we use some machine learning techniques like tokenization. Extract some news feed articles from newsapi and store them in a csv file. Ingest them in a pandas data frame. Preprocess the extracted data using tf-idf algorithm and use tokenization to convert them into tokens, to get interesting results.
\end{abstract}

Index Terms - Tf-idf algorithm, Tokenization 


\title{
International Journal for Innovative
} Engineering and Management Research

A Peer Revieved Open Access International Journal

\section{A novel graphical password authentication mechanism for cloud services}

\author{
Kameswara Rao, M., Usha Switha, T., Naveen, S. \\ Department of ECM, Koneru Lakshmaiah Education Foundation, \\ Green Fields, Vaddeswaram, A.P., India-522502.
}

\begin{abstract}
:
Password provides high security and confidentiality for the data and also prevents unauthorized access. So, the most popular authentication method which is the alphanumeric passwords that provides security to users which are having strings of letters and digits. Due to various drawbacks in text based passwords, graphical password authentication is developed as an alternative. In graphical password authentication, password is provided based on the set of images. For users it is easy to remember images than text and also graphical passwords provide more security when compared to text based. There are two techniques in graphical passwords. They are Recognition based technique and Recall based technique. To provide more security to user a new idea has been proposed by combining Recognition based and Recall based techniques in this paper
\end{abstract}

Index Terms - Authentication, Graphical password, Recall based technique, Recognition based technique.enization 


\title{
A graphical password authentication system for touch screen based devices
}

\author{
Kameswara Rao, M., Aparna, P., Avinash Akash, G. \\ Department of ECM, Koneru Lakshmaiah Education Foundation, \\ Green Fields, Vaddeswaram, A.P., India-522502.
}

\begin{abstract}
:
Mobile devices equipped with touch screens dominate the current mobile market because of high flexibility and good usability. Many privacy sensitive applications run on such devices. For authentication typing a text password is challenging task in touch screen based devices. A graphical based password is one of the most promising and upcoming alternative for textual based passwords. According to psychology, human's can remember pictures more easily than text. In this paper we present a novel graphical password authentication system which is a combination of both recall and recognition based techniques specifically for touch screen based mobile and handheld devices. A user study was conducted to explore the usability and security of the proposed scheme.
\end{abstract}

Index Terms - Authentication, Graphical password, Recall based technique, Recognition based technique. 

Engineering and Management Research

A Peer Revieved Open Access International Journal

\title{
Random grid size based graphical password authentication
}

\author{
Apoorva, N., Lokesh, P.N., Kameswara Rao, M. \\ Department of ECM, Koneru Lakshmaiah Education Foundation, \\ Green Fields, Vaddeswaram, A.P., India-522502.
}

\begin{abstract}
:
Secret word presents tall security and secrecy for the measurements and also anticipates unauthorized get affirmation to. In graphical watchword confirmation, secret word is provided basically based on the set of pictures. For clients it is direct to consider photographs than literary substance and also graphical passwords offer additional security while in comparison to literary substance based. There are two procedures in graphical passwords there are notoriety basically based strategy and take into account based approach. To supply more security to individual an unused concept has been proposed by way of combining notoriety based completely and review basically based on methodologies.
\end{abstract}

Index Terms - Graphical password, Grid Based, Session Authentication 


\title{
Defected Ground Structure Switchable Notch Band Antenna for UWB Applications
}

\author{
${ }^{1}$ Vamseekrishna Allam, ${ }^{2}$ B. T. P. Madhav \\ ${ }^{1}$ Department of ECM, Koneru Lakshmaiah Education Foundation, \\ Green Fields, Vaddeswaram, A.P., India-522502. \\ ${ }^{2}$ Department of ECE, Koneru Lakshmaiah Education Foundation, \\ Green Fields, Vaddeswaram, A.P., India-522502.
}

\begin{abstract}
A defected ground structure notch band antenna is proposed in this work. The switchable characteristics for the designed notch band antenna are achieved through open-end slots on/off positions. The proposed DGS notch band antenna is capable of notching the frequency bands 3-4, 5.5-6.5 GHz, respectively. A high notch band rejection with VSWR greater than 2 and the return loss greater than $-10 \mathrm{~dB}$ is achieved at the notching frequencies. The defected ground structure is providing balance in the impedance bandwidth to the designed models. By sorting the slots on the radiating structure, the tunability in the notching frequencies is attained in this paper. The antenna radiation characteristics and the surface current distributions at operating bands as well as at notch bands are presented in this work. The proposed notch band antenna is providing high rejection of gain in the notch band and average gain of $2.8 \mathrm{~dB}$ in the operating band.
\end{abstract}

Keywords : Defected ground structure, Monopole antenna, Notch band Switchability, Ultrawideband 


\title{
Planar Switchable Notch Band Antenna with DGS for UWB Applications
}

\author{
${ }^{1}$ Vamseekrishna Allam, ${ }^{2}$ B. T. P. Madhav \\ ${ }^{1}$ Department of ECM, Koneru Lakshmaiah Education Foundation, \\ Green Fields, Vaddeswaram, A.P., India-522502. \\ ${ }^{2}$ Department of ECE, Koneru Lakshmaiah Education Foundation, \\ Green Fields, Vaddeswaram, A.P., India-522502.
}

\begin{abstract}
A planar notch band antenna with a defected ground structure is proposed in this work. The switchable characteristics for the designed notch band antenna are achieved through open end slots on/off positions. The proposed notch band antenna is capable of notching the frequency bands 3-4 GHz, 5.5-6.5 GHz respectively. A high notch band rejection with VSWR greater than 2 and the return loss greater than $-10 \mathrm{~dB}$ is achieved at the notching frequencies. The defected ground structure provides balance in the impedance bandwidth to the designed models. By sorting the slots on the radiating structure, the tunability in the notching frequencies are attained in this paper. The antenna radiation characteristics and the surface current distributions at operating bands as well as at notch bands are presented in this work. The proposed notch band antenna is providing high rejection of gain in the notch band and average gain of $2.8 \mathrm{~dB}$ in the operating band.
\end{abstract}

Keywords: Defected ground structure $\cdot$ Monopole antenna $\cdot$ Notch band, Switchability $\cdot$ Ultrawideband 


\title{
International Journal for Innovative Engineering and Management Research
}

A Peer Revieved Open Access International Journal

\section{Smart Restaurants}

\section{${ }^{1}$ Vamseekrishna Allam, ${ }^{2}$ B. T. P. Madhav}

${ }^{1}$ Department of ECM, Koneru Lakshmaiah Education Foundation,

Green Fields, Vaddeswaram, A.P., India-522502.

${ }^{2}$ Department of ECE, Koneru Lakshmaiah Education Foundation, Green Fields, Vaddeswaram, A.P., India-522502.

\begin{abstract}
Conventional strategy that is utilized usually in hotels/restaurants is by taking the client's requests and recording it on a bit of paper and afterward giving the request in the kitchen area.The nourishment requesting framework is proposed with the utilization of a handheld gadget set on each table which is utilized to make a request at the eatery. The framework utilizes a push buttons in addition to show module which is put on every client table for them to make orders. Request is made by choosing the things showed on the mobile App. The request will be sent from the client segment utilizing ZigBee correspondence, and consequently will be shown on a screen at the kitchen. The bill will be shown with table number at the administrator/charging area. The task will lessen the time spent on making the requests and paying the bills, whereby the cost and labor likewise can be diminished.
\end{abstract}

Keywords: Smart restaurants; Mobile Application; Cloud Computing Architecture, IoT 


\title{
International Journal for Innovative Engineering and Management Research
}

A Peer Revieved Open Access International Journal

\section{RECONFIGURABLE NOTCH BAND ANTENNA USING PIN DIODES}

\author{
${ }^{1}$ Vamseekrishna Allam, ${ }^{2}$ B. T. P. Madhav, ${ }^{1}$ Y. Nagarjuna, ${ }^{1}$ S. \\ Lakshmi Manasa, ${ }^{1}$ V. Mourya, ${ }^{1}$ Y. Yaswant \\ ${ }^{1}$ Department of ECM, Koneru Lakshmaiah Education \\ Foundation, Green Fields, Vaddeswaram, A.P., India- \\ 522502. \\ ${ }^{2}$ Department of ECE, Koneru Lakshmaiah Education \\ Foundation, Green Fields, Vaddeswaram, A.P., India-
} 522502.

\begin{abstract}
A reconfigurable notch band antenna is presented with the help of pin diodes. Generally different bands are required to achieve different functionalities using an antenna as each application requires a specific band. To implement all frequencies through a single antenna notch filters can be used. Notch filters reject the frequencies which are undesired. To implement different notch functions in micro strip antenna, different slots are made on the patch and on the ground plane. Each slot will hold the responsibility for a different narrow band frequency. As all the frequencies are nearer and narrow bands, they may suffer interference. Each slot will suffer mutual coupling because of it. To reduce such effect different shapes for slots are selected. In order to achieve reconfiguration in the notch band antenna we have added PIN diodes with the help of lumped RLC to control these slots.
\end{abstract}

Keywords: Reconfigurable, PIN Diodes, notch band antenna, slots, micro strip antenna. 